Copyright

by

Jasmine Star Yuko Ma Ratchford

2012 
The Dissertation Committee for Jasmine Star Yuko Ma Ratchford certifies that this is the approved version of the following dissertation:

\section{Identifying Muons for Neutrino Oscillation and Cross Section Experiments}

Committee:

Sacha Kopp, Supervisor

Christopher Sneden

Christina Markert

Jack Ritchie

Karol Lang 


\title{
Identifying Muons for Neutrino Oscillation and Cross Section Experiments
}

\author{
by \\ Jasmine Star Yuko Ma Ratchford, B.S.A. \\ DISSERTATION \\ Presented to the Faculty of the Graduate School of \\ The University of Texas at Austin \\ in Partial Fulfillment \\ of the Requirements \\ for the Degree of \\ DOCTOR OF PHILOSOPHY
}

THE UNIVERSITY OF TEXAS AT AUSTIN

May 2012 
For my husband, Daniel. 


\section{Acknowledgments}

I have many wonderful people to thank for the success of this thesis and the happiness I found in graduate school.

First, I would like to thank my advisor, Sacha Kopp, for his excellent advice and constant support throughout. I was extremely lucky to have had an advisor who was so knowledgeable about many different subjects and was so enthusiastic about the work I completed.

Rustem Ospanov, my muon identification predecessor, deserves a special thanks for both laying the foundations of the work I was to continue and for being great friend.

There were many, many people who made the MINOS charged current analysis possible and all of these people deserve thanks (thanks!). However, Prof. Karol Lang, Ryan Nichol, and Justin Evans deserve particular recognition for being a great source of advice and supervising the group well.

I would also like to thank everyone from the Texas neutrino groups that I haven't thanked yet. Zarko Pavlovic and Laura Loiacono helped me get started when I first began at UT, and Melissa Jerkins and Parker Cravens were the best office mates one could ask for.

I would especially like to thank those teachers and mentors who have had a profound influence on me, including Helmut Vogel, Janet Conrad, and Irv Eisenberg.

Finally, I would like to thank my family. My parents for their love and 
support, my sister for being a confidant and great friend, and, of course, my husband who was by far the best thing about graduate school.

Daniel, you are my best friend and I could not have done this without you. 


\title{
Identifying Muons for Neutrino Oscillation and Cross Section Experiments
}

\author{
Publication No. \\ Jasmine Star Yuko Ma Ratchford, Ph.D. \\ The University of Texas at Austin, 2012
}

Supervisor: Sacha Kopp

Neutrinos $(\nu)$ are interesting for many reasons; they are the only fundamental fermions which are electrically neutral; their mass is orders of magnitude smaller than the lightest charged lepton, the electron; and their solely weak interactions make them an excellent probe of the weak nuclear force. However, one of the most interesting aspects of neutrinos is that, unlike their charged lepton partners, neutrino mass and flavor eigenstates are not the same. All leptons possess 'lepton flavor', a property which is conserved in neutrino interactions. However, because of the difference in the mass and weak eigenstates of neutrinos, a quantum-interference effect is seen in the time evolution of neutrinos. This results in energy and distance dependent oscillations of the neutrino's lepton flavor called 'neutrino oscillations'.

The MINOS experiment (Main Injector Neutrino Oscillation Search) was designed to measure the neutrino oscillation parameters, $\Delta m_{32}^{2}$ and $\sin ^{2}\left(2 \theta_{32}\right)$. MINOS is composed of two detectors located on a 'beam' of $\nu_{\mu}$ s. The MINOS Near Detector is located at Fermilab, and the Far Detector is located at the 
Soudan Mine in Minnesota, $734 \mathrm{~km}$ after the Near Detector. The MINER $\nu \mathrm{A}$ experiment (Main Injector Neutrino Experiment for $\nu-A$ ) is a neutrino experiment placed directly in front of the MINOS Near Detector. MINER $\nu$ A's goal is to make precision measurements of neutrino cross sections. This will help with uncertainties in oscillation measurements, such as MINOS' at low energy.

Although lepton flavor is conserved in neutrino interactions, the final state lepton can be a charged lepton ('charged current' interactions) or a neutrino ('neutral current' interactions) of a particular flavor. The identification of charged current $\nu_{\mu}$ interactions through the identification of a muon in the final state is a critical component to both neutrino oscillation and cross section measurements; neutral current events are a background to the oscillation signal because the properties of the incoming neutrino cannot be determined. Such identification is particularly difficult and important for low-energy neutrino events.

In this thesis, we will discuss improvements to the MINOS charged current identification at low energies, studies to estimate the effect of the neutral current background on the measurement of the oscillation parameters, and the aspects of muon identification which are similar for the MINOS and MINER $\nu \mathrm{A}$ experiments. In 2010, the MINOS experiment released a measurement of the oscillation parameters based on $7.32 \times 10^{20}$ POT. The results were $\Delta m_{32}^{2}=2.32_{0.08}^{+0.12} \times 10^{3} \mathrm{eV}^{2}$, and $\sin ^{2}\left(2 \theta_{32}\right)>0.90(90 \%$, C.L. $)$. This is the best measurement of the oscillation parameter, $\Delta m_{32}^{2}$, and a competitive measurement of $\sin ^{2}\left(2 \theta_{32}\right)$. The improvements to the charged current event selection helped MINOS observe a complete oscillation in neutrino energy.

viii 


\section{Table of Contents}

Acknowledgments $\quad$ v

Abstract vii

List of Tables $\quad$ xiii

List of Figures $\quad$ xiv

Chapter 1. Neutrino Physics $\quad 1$

1.1 Foundations of Neutrino Physics . . . . . . . . . . . . 2

1.2 Neutrino Mass and Oscillations . . . . . . . . . . . . . . 4

1.2.1 Neutrino Oscillation Theory . . . . . . . . . . . . 4

1.2.2 The Two-Flavor Approximation . . . . . . . . . . 7

1.2.3 First Evidence of Neutrino Oscillations . . . . . . . . . . 9

1.3 Neutrino Interactions . . . . . . . . . . . . . . . . 16

1.3.1 Neutrino Scattering in the Standard Model . . . . . . . 16

1.3.2 Neutrino Scattering Off Hadrons . . . . . . . . . . . 20

1.3.3 Neutrino Scattering off of Nuclei . . . . . . . . . . 26

1.4 An Overview of This Thesis . . . . . . . . . . . . . . . 31

Chapter 2. The NuMI beamline 33

2.1 The NuMI Beam Facility . . . . . . . . . . . . . . . . . . 33

2.1.1 Run Conditions leading up to the MINOS Charged Current Analysis . . . . . . . . . . . . . . . 42

2.2 Predicting the NuMI Flux at the Near Detector . . . . . . . . 42

2.3 The MINOS Near-to-Far Extrapolation and the General Fitting Method .................. 46 
Chapter 3. The MINOS and MINER $\nu$ A detectors 56

3.1 Neutrino Detectors on the NuMI beamline . . . . . . . . 56

3.2 Charged Particle Interactions with Matter . . . . . . . . 57

3.3 The MINOS Detectors . . . . . . . . . . . . . . 63

3.3.1 The MINOS Fiducial Volumes . . . . . . . . . . . 74

3.4 The MINER $\nu$ A Detector . . . . . . . . . . . . 75

Chapter 4. MINOS Charged Current Event Identification $\quad 89$

4.1 Previous Algorithms for Muon Identification . . . . . . . . 90

4.2 The Challenges of Muon Identification at Low Energy . . . . . 94

4.3 Identifiers of Low-Energy Muons . . . . . . . . . . . . . . 99

Chapter 5. The Neutral Current Background 112

5.1 Estimating the Neutral Current Background . . . . . . . . . . 113

5.2 Muon Removal Procedure . . . . . . . . . . . . . . . . . . 118

5.3 The Neutral Current Background Shape . . . . . . . . . . . . . 124

5.4 The Neutral Current Systematic Error . . . . . . . . . . . 128

5.4.1 Using the kNN Shower Energy Estimate . . . . . . . . 136

5.5 The Neutral Current Background Normalization Error . . . . . 140

5.6 Summary . . . . . . . . . . . . . . . . . . . . . . . 143

Chapter 6. Optimization of the Charged Current Selection Threshold 147

6.1 Model Sensitivity . . . . . . . . . . . . . . . . . . . . . . . 149

6.2 Oscillation Sensitivity _. . . . . . . . . . . . 152

6.3 Neutral Current Contamination . . . . . . . . . . . . 157

Chapter 7. Improvements to the MINOS $\nu_{\mu}$ Disappearance Analysis 169

7.1 Previous Measurements of Oscillation Parameters Through $\nu_{\mu}$ Disappearance by MINOS . . . . . . . . . . . . . . . . . 169

7.2 Other Analysis Improvements . . . . . . . . . . . . . . 172

7.2.1 A k-NN based Shower Energy Estimate . . . . . . . . . 173

7.2.2 The Rock and Anti-Fiducial Analysis . . . . . . . . . . 175

7.2 .3 Resolution Binning . . . . . . . . . . . . . . . . . . 181

7.3 Summary of the Analysis Improvements . . . . . . . . . . 185 
Chapter 8. Results from the 2010 MINOS $\nu_{\mu}$ Disappearance Analysis

8.1 Calculation of Systematic Errors . . . . . . . . . . . 187

8.2 The Fitting Procedure . . . . . . . . . . . . . . . . 191

8.3 Far Detector Data Distributions and Statistics . . . . . . . . 193

8.4 Measurements of $\nu_{\mu}$ Disappearance in the Far Detector . . . 203

Chapter 9. Charged Current $\nu_{\mu}$ Identification in Minerva 211

9.1 Hadronic Particle Identification in MINER $\nu \mathrm{A}$. . . . . . . . . 212

9.2 Muon Identification using a MINOS k-NN approach . . . . . . 213

9.2.1 Categories of Particles in MINER $\nu \mathrm{A}$. . . . . . . . . . 215

9.2.2 Muon Identification Variables in the MINER $\nu \mathrm{A}$ Tracker, ECAL and HCAL regions . . . . . . . . . . . . 220

9.2.3 Muon Identification Variables in the MINER $\nu$ A Outer Detector . . . . . . . . . . . . . . 223

9.2.4 MINER $\nu \mathrm{A}$ Region $\mathrm{k}-\mathrm{NNs}$ and an Aggregate Score for Muon Identification . . . . . . . . . . . . . . 226

9.3 Muon Energy Estimates for the MINER $\nu$ A Experiment . . . . 233

9.4 Future Relevant MINER $\nu$ A charged current studies . . . . . . 236

$\begin{array}{lll}\text { Chapter 10. Summary and Conclusions } & 239\end{array}$

10.1 Summary . . . . . . . . . . . . . . . . . . . . 239

10.2 Future Neutrino Measurements . . . . . . . . . . . . . . . 240

10.3 Final Thoughts on Neutrinos . . . . . . . . . . . . . . . 243

$\begin{array}{ll}\text { Appendices } & 245\end{array}$

Appendix A. Using a k-Nearest Neighbor Method for Identification and Extrapolation 246

A.1 Description of the k-NN Algorithm _ . . . . . . . . . 246

A.2 Running Time and the k-D Tree . . . . . . . . . . . . . 251

A.3 The Number of Nearest Neighbors and dimensionality . . . . 254

A.4 Other Multivariate Techniques . . . . . . . . . . 256 
Appendix B. Low-Energy events added by the CC/NCSeparator 258

B.1 ADC colored plots . . . . . . . . . . . . . 258

B.2 Track and shower hit plots ............ 265

Appendix C. The Neutral Current Background Study: Distributions from Daikon04/Dogwood1

273

$\begin{array}{ll}\text { Bibliography } & 280\end{array}$

xii 


\section{List of Tables}

1.1 A selective (and incomplete) list of neutrino oscillation measurements from SNO, T2K, SuperK and MINOS. The values represented in this table are the single-experiment best limits for their respective variable. . . . . . . . . . . . . . 12

2.1 The MINOS experiment data accumulation summary. . . . . . 43

5.1 Results from previous studies . . . . . . . . . . . . . 117

5.2 The difference in POT count from the original and muon-removed charged current files . . . . . . . . . . . . . . 122

5.3 Normalization values for neutral current and charged current Monte Carlo before and after re-weighting . . . . . . . . . . . 142

6.1 Statistical and systematic error increases for different thresholds 166

7.1 Systematic Uncertainties for the Previous MINOS measurement 170

8.1 A table of the primary systematic errors for the MINOS $\nu_{\mu}$ disappearance measurement . . . . . . . . . . . . . . 190

8.2 Catalog of the number of events after different selections . . . 196

8.3 Events gained in the Far Detector by the $2010 \mathrm{CC} / \mathrm{NC}$ separation thresholds below $3 \mathrm{GeV}$. . . . . . . . . . . . . . 200

8.4 Events expected and observed at the Far Detector by run (fidu-

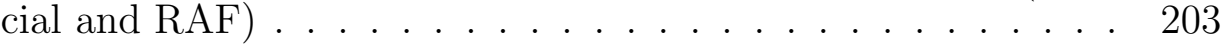

8.5 Statsistics-only fits of Run I and Run II fiducial data. This table shows the effect of the new k-NN shower energy estimate and separation parameter thresholds have on the previous result.

8.6 The Far Detector fully reconstructed (fiducial) events by resolution bin and run number . . . . . . . . . . . . . 206

C.1 Normalization values for neutral current and charged current Monte Carlo before and after re-weighting . . . . . . . . . . 279 


\section{List of Figures}

1.1 The two-neutrino approximation probability for $\nu_{\mu} \rightarrow \nu_{\mu}$ transition over a $735 \mathrm{~km}$ baseline . . . . . . . . . . . . 8

1.2 SuperKamikande 1998 result . . . . . . . . . . . . . . . . . . 13

1.3 Fermi four-point interaction Feynman diagram . . . . . . . . . 17

1.4 Feynman diagram of a charged current and neutral current event ...................... 20

1.5 Total and inclusive cross-sections from Paulo Lipari. . . . . . . 22

1.6 Feynman diagram of a charged current quasi-elastic event . . . 23

1.7 Feynman diagram of a charged current resonant event . . . . . 24

1.8 Feynman diagram of deep-inelastic scattering event . . . . . . 26

1.9 A example depiction of how final state interactions can modify a neutrino event . . . . . . . . . . . . . 27

1.10 Multiplicity measurements from the 15-foot experiment at FNAL and the BEBC experiment at CERN . . . . . . . . . . . 29

2.1 The NuMI beamline drawing by Robert Zwaska . . . . . . . . 34

2.2 The NuMI horns (diagram) . . . . . . . . . . . . . 37

2.3 The NuMI LE010185N beam flux at the MINOS Near Detector decomposed into focusing subcategories . . . . . . . . 38

2.4 The NuMI flux . . . . . . . . . . . . . . . . . . . . . 41

2.5 The charged pion ratio estimate from the MINOS Near Detector data ......................... 45

2.6 The kinematic distributions of $\pi^{+} / K^{+}$production off the NuMI target before and after tuning . . . . . . . . . . 47

2.7 The Near Detector neutrino energy spectrum . . . . . . . . . . 48

2.8 Near-to-Far Extrapolation . . . . . . . . . . . . . . . . . . 49

2.9 The Beam Matrix for $\nu_{\mu} \ldots \ldots$. . . . . . . . . . . . 52

3.1 dEdX curves for Iron . . . . . . . . . . . . . . . . . . 59

3.2 A demonstration of the Landau distribution . . . . . . . . . 60 
3.3 the measured energy loss in the MINOS detectors . . . . . . 62

3.4 The NuMI beam from Fermilab to the Soudan Mine- diagram 65

3.5 The MINOS Near Detector . . . . . . . . . . . . . . . 68

3.6 The MINOS Far Detector . . . . . . . . . . . . . . . 69

3.7 MINOS Event Display : charged current DIS . . . . . . . . . 70

3.8 MINOS Event Display: charged current QEL . . . . . . . . . 71

3.9 MINOS Event Display: neutral current DIS . . . . . . . . . 72

3.10 MINOS Event Display: neutral current DIS \#2 . . . . . . . . 73

3.11 3D MINER $\nu$ A detector diagram . . . . . . . . . 76

3.12 A MINER $\nu$ A detector module . . . . . . . . . . . 78

3.13 Coordinate systems of the MINOS and MINER $\nu$ A detectors. . 79

3.14 2D MINER $\nu$ A detector diagram . . . . . . . . . . . 81

3.15 The MINER $\nu$ A detector as constructed. . . . . . . . . . . 82

3.16 MINER $\nu$ A Event Display: charged current QEL . . . . . . . 85

3.17 MINER $\nu$ A Event Display: muon leaves through the OD $\ldots 86$

3.18 MINER $\nu$ A Event Display: neutral current DIS . . . . . . . . 87

3.19 MINER $\nu$ A Event Display: charged current DIS . . . . . . . 88

4.1 Muon discriminating variables as used in the 2008 MINOS analysis 93

4.2 Data to Monte Carlo comparisons for the muon discriminating variables used in $\mu \mathrm{ID}$-A. . . . . . . . . . . . . . 95

$4.3 \mu \mathrm{ID}-\mathrm{A}$ or "CC/NC separation parameter" . . . . . . . . 96

4.4 The $\mu$ ID-A variables with track lengthi20 planes . . . . . . . 100

4.5 The percent of events missed by the $\mu$ ID-A by energy . . . . 101

4.6 A short track with an nuclear interaction. . . . . . . . . . . 102

4.7 A short track which is muon like . . . . . . . . . . . . 103

4.8 The low energy muon variables - Area Normalized . . . . . . . 107

4.9 Low-energy muon variables - ND Monte Carlo and data . . . . 108

4.10 The low-energy muon variaqbles for tracks with less than 20 planes and reconstructed energy $i 3 \mathrm{GeV}$. . . . . . . . . . . 109

$4.11 \mu \mathrm{ID}-\mathrm{B}$, or "CC/NC separation parameter(low energy)" . . . . 111

5.1 Feynman diagram of a charged current and neutral current event . . . . . . . . . . . . . . . . . . 114 
5.2 An event before muon removal . . . . . . . . . . . . . 120

5.3 An event after muon removal . . . . . . . . . . . . . . . . 121

5.4 The muon-removed charged current reconstructed neutrino energy and inelasticity . . . . . . . . . . . . . . 123

5.5 Parent Event Inelasticity for different POT countings . . . . . 125

5.6 Distributions for muon-removed charged current events derived from events with a $\mu \mathrm{ID}-\mathrm{A}>0.3$ and true Monte Carlo neutral current events . . . . . . . . . . . . . . 126

5.7 Comparisons of neutral current and muon-removed charged currentlike events . . . . . . . . . . . . . . . . . . 127

$5.8 \mathrm{kNN}$ distributions for muon-removed charged current events. These kNNs together compose the 2010 PID . . . . . . . . . 129

5.9 muon-removed charged current data and Monte Carlo Energy

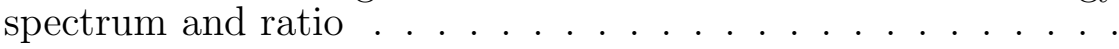

5.10 muon-removed charged current data and Monte Carlo inelasticity distribution and ratio .................

5.11 The reweighted neutral current background distributions (Energy and Inelasticity) . . . . . . . . . . . . . . .

5.12 Ratio of muon-removed charged current data nd Monte Carlo in bins of energy and inelasticity (simultaneously) . . . . . . .

5.13 MRCC data and Monte Carlo distributions using kNN shower energy . . . . . . . . . . . . . . .

5.14 neutral current and muon-removed charged current Monte Carlo distributions using kNN shower energy . . . . . . . . . . . 138

5.15 The re-weighted neutral current background with the kNN shower energy . . . . . . . . . . . . . . . .

5.16 MC (charged current,neutral current and Total) and data before and after re-weighting for overall best fit, with derived thresholds . . . . . . . . . . . . . . . . .

5.17 MC (charged current,neutral current and Total) and after reweighting for overall best fit, with derived thresholds . . . . . 145

6.1 Difference in log-likelihood of decay and oscillation hypotheses. 151

6.2 Difference in log-likelihood for decoherence and oscillation hypotheses . . . . . . . . . . . . . . . . 151

6.3 Log-likelihood for oscillation hypothesis . . . . . . . . . 152

6.4 Oscillation contours for various charged current selections. . . 154 
6.5 Likelihoods at non-best-fit oscillation parameters . . . . . . . 156

6.6 Total efficiencies and purities at a given $\mathrm{kNN}$ cuts for events with energy less than $2 \mathrm{GeV}$. . . . . . . . . . . . . 158

6.7 Efficiency versus reconstructed energy for different selection thresholds . . . . . . . . . . . . . . . . . 160

6.8 Purity versus reconstructed energy for different selection thresholds . . . . . . . . . . . . . . . . . . . . . 162

6.9 Efficiency $\times$ purity versus reconstructed energy for different selection thresholds . . . . . . . . . . . . . . . . 163

6.10 Total neutral current background. Comparing the 2008 MINOS $\nu_{\mu}$ disappearance selection to other selection thresholds. . . . . 165

6.11 Efficiency versus $\nu_{\mu}$ energy for the ND and FD . . . . . . . . 168

7.1 The ratio of the Far Detector data to the unoscillated prediction 171

7.2 Systematic error 'Star Plot' . . . . . . . . . . . . . . . . . . . 172

7.3 the k-NN shower energy estimate before the polynomial correction 174

7.4 Comparison of the calorimetric and k-NN shower energy estimates176

7.5 Sensitivity contours for the k-NN shower energy estimate . . 177

7.6 The anti-fiducial regions of the Far Detector. . . . . . . . . . . 178

7.7 The true neutrino energy for RAF events . . . . . . . . . . . . 180

7.8 The reconstructed muon energy versus true neutrino energy for RAF events ..................... . 181

7.9 The RAF analysis sensitivity contours . . . . . . . . . . . . . 182

7.10 The resolution binning parametrization . . . . . . . . . . 183

7.11 Resolution Quantiles for the 2010 MINOS $\nu_{\mu}$ disappearance

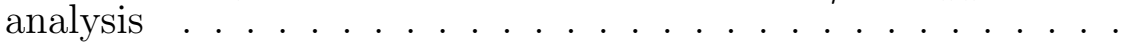

7.12 The reconstructed energy versus the true energy for events in (a) The highest resolution bin and (b) the lowest resolution bin. 185

7.13 The changes in sensitivity produced by shower energy estimator, resolution binning and inclusion of rock and anti-fiducial events. Each analysis improvement reduces the allowable space of the oscillation analysis. . . . . . . . . . . . . . . 186

8.1 Systematic shifts to the best-fit oscillation parameters . . . . . 191

8.2 The effect of systematic uncertainties on the sensitivity to decay and decoherence . . . . . . . . . . . . . . . . . 192 
8.3 Position of Far Detector fiducial events . . . . . . . . . . . . 195

8.4 CC/NC separation . . . . . . . . . . . . . . . . . 197

$8.5 \mu \mathrm{ID}-\mathrm{B}$ variable for the Far Detector $\ldots \ldots \ldots$

8.6 The events gained by new selection in resolution bins. . . . . . 199

8.7 The events gained by the new thresholds for charged current selection by selection values . . . . . . . . . . . . . . . 201

8.8 The events gained by the new analysis of the charged current selection by reconstructed energy . . . . . . . . . . . . . 202

8.9 The Far Detector energy spectrum in resolution bins with the best-fit oscillation result . . . . . . . . . . . . . . 205

8.10 The Far Detector energy spectrum for fully reconstructed charged current events . . . . . . . . . . . . . . . 207

8.11 The Far Detector energy spectrum for partially reconstructed charged current events . . . . . . . . . . . . . . . 208

8.12 Contour results using fiducial and RAF events . . . . . . . . 210

9.1 The average dEdX value for pions and protons 2 to $7 \mathrm{~cm}$ from the end of the track . . . . . . . . . . . . . . . . . . . . 214

9.2 The muon energy spectrum for the MINER $\nu \mathrm{A}$ experiment(Monte Carlo $\ldots \ldots \ldots \ldots$. . . . . . . . . . . . . . . . . 219

9.3 The Average Cluster Energy (Tracker Region) divided by prong type . . . . . . . . . . . . . . . . 220

9.4 Muon identification variables for the MINER $\nu$ A Tracker region 222

9.5 Muon identification variables for the MINER $\nu$ A ECAL region 224

9.6 Muon identification variables for the MINER $\nu$ A HCAL region 225

9.7 The MINER $\nu$ A Outer Detector muon-identification variables . 227

9.8 The Sub-detector k-NN muon identifiers for the MINER $\nu \mathrm{A}$ experiment . . . . . . . . . . . . . . . 228

9.9 The MINER $\nu$ A muon-identification score. . . . . . . . . 230

9.10 The muon energy spectrum in MINER $\nu \mathrm{A}$ after a $\mathrm{k}-\mathrm{NN}$ based muon selection. . . . . . . . . . . . . . . . . 231

9.11 The efficiency and contamination of a MINOS-like muon particle identifier . . . . . . . . . . . . . . . . . . 232

9.12 The percent difference between reconstructed and true energy for MINOS matched MINER $\nu$ A muons . . . . . . . . . . . 234

9.13 The energy of ODMatched muons by story . . . . . . . . 235 
$10.1 \mathrm{NO} \nu \mathrm{A}$ event displays . . . . . . . . . . . . . . . . 241

10.2 The expected event rate at $\mathrm{NO} \nu \mathrm{A} . \ldots \ldots \ldots$

A.1 k-Nearest-Neighbors: a 2-Dimensional example . . . . . . . . . 249

A.2 Demonstration of k-NN algorithm versus querying within a particular radius . . . . . . . . . . . . . . . 251

A.3 An example of a k-D tree. . . . . . . . . . . . . . 253

C.1 Area normalized distributions of the neutral current and muonremoved charged current energy and inelasticity distributions 274

C.2 Inelasticity distribution for Daikon04/Dogwood1 muon-removed charged current data and Monte Carlo events . . . . . . . . . 275

C.3 The muon-removed charged current data/Monte Carlo ratio versus reconstructed energy and inelasticity for Daikon04, Dogwood3 Monte Carlo and reconstruction. . . . . . . . . . . 276

C.4 The normalization study using "Daikon04/Dogwood1" Monte Carlo and reconstruction . . . . . . . . . . . . . . 278 


\section{Chapter 1}

\section{Neutrino Physics}

Neutrinos are one of the most abundant particles in the universe, with 100 million relic neutrinos per cubic meter from the big bang, and roughly $10^{14}$ neutrinos from the Sun pass through a person every second [1]. However, neutrinos only interact through the weak nuclear force, and, therefore, do not readily interact with other particles. As a result, neutrinos are particularly difficult to study. Neutrino experiments require extremely massive detectors and use high-intensity sources in order to produce statistically significant results. With so little comparitively known about neutrinos, neutrino experiments are integral to understanding weak nuclear interactions and are an important probe of new physics.

In 1968, the Homestake experiment published a strange result on the production rate of neutrinos in the Sun [2]. The Homestake experiment found that neutrinos from the Sun seemed to arrive in deficit relative to the flux anticipated from solar luminosity. Eventually, it was found that the best model for this apparent deficit is "neutrino oscillations" [3-5]. The oscillation theory predicts that the neutrinos are changing "flavor" (or neutrino type) as they travel from the Sun. Because these oscillated neutrinos were undetectable by the Homestake experiment, an apparent deficit was seen. Verification of the neutrino oscillation hypothesis has since been the primary focus of many neutrino experiments. 
The primary goal of the work presented in this thesis is improvements to the study of muon neutrino oscillations using the MINOS experiment at the Fermi National Accelerator Laboratory. In this chapter, we will discuss the underlying physics of neutrino experiments beginning with a summary of the Standard Model. This will be followed by a discussion of neutrino oscillations and interactions. We will briefly discuss nuclear effects which can skew kinematic distributions of neutrino interactions. Finally, we review the experiemntal methods to be employed and developed in the remainder of this thesis.

\subsection{Foundations of Neutrino Physics}

The generally accepted interpretation of particle physics phenomena is called the "Standard Model" [6-8]. The Standard Model is composed of two different theories, both of which describe subatomic phenomena as the interaction between point-like particles obeying fermi-dirac statistics (fermions) and fields mediated by particles which obey bose-einstein statistics (bosons). The elementary fermions can be divided into two groups, leptons and quarks. Quarks are defined as fermions which posess color quantum number and, therefore, can interact through the strong force. Leptons lack the color quantum number and thus only interact through the electroweak force. Because the strong force does not allow for a quark to be isolated, quarks are found only in quark-anti-quark pairs (mesons) or 3-quark integer electric charge combinations (baryons). Leptons are further divided into "charged" leptons (the electron,e, the muon, $\mu$, and tau, $\tau$ ) and neutrinos (the electron neutrino $\nu_{e}$, muon neutrino, $\nu_{\mu}$, and tau neutrino, $\left.\nu_{\tau}\right)$. The distinguishing trait of neutrinos is that they are the only fundamental fermions that have no electric charge. In 
contrast, quarks have fractional charges $(+2 / 3$, and $-1 / 3)$ and charged leptons have unit charge (-1), measured in terms of $e=1.6 \times 10^{-19}$ Coulombs.

There are four gauge bosons which mediate interactions. The mediator of strong interactions, the gluon, is outside the scope of this thesis. The remaining gauge bosons are the photon, which mediates electromagnetic interactions, and the $W^{ \pm}$and $Z^{0}$ bosons, which mediate weak interactions. Electroweak theory, which describes these interactions, is discussed in Section 1.3.1.

Quarks and leptons are traditionally grouped into three different "generations". Each generation contains an up-type (+2/3 charged) quark, a down-type (-1/3 charged) quark, a charged lepton (-1 charged), a neutrino, and their anti-particles. The first generation contains the lightest mass quarks and charged lepton. ${ }^{1}$ Atomic matter is made from first-generation particles, including the up (u) and down (d) quarks, and the electron.

Although there is no relation between the quark and lepton generations, leptons within a generation share a quantum number called "flavor" which is conserved in all interactions amoung particles, including weak interactions. ${ }^{2}$ Each flavor is named after the charged lepton in the interaction. The three lepton flavors and charged leptons are named electron (e), muon $(\mu)$, and tau $(\tau)$. The neutrinos associated with each flavor are named the electron neutrino $\left(\nu_{e}\right)$, the muon neutrino $\left(\nu_{\mu}\right)$, and the tau neutrino $\left(\nu_{\tau}\right)$ respectively. The electron is the lightest charged lepton, with a mass $m=0.5109 \mathrm{MeV} / \mathrm{c}^{2}$,

\footnotetext{
${ }^{1}$ The neutrino masses are unknown and do not share a one-to-one correspondence with lepton flavor states. This is a crucial fact in neutrino oscillations.

${ }^{2}$ Flavor is conserved in the Standard Model. While neutrino oscillation theory violates lepton number, it does not involve interactions with other particles through the Standard Model.
} 
followed by muon, $m_{\mu}=105.7 \mathrm{MeV} / \mathrm{c}^{2}$, with the tau being the heaviest lepton, $m_{\tau}=1.777 \mathrm{GeV} / \mathrm{c}^{2}$.

\subsection{Neutrino Mass and Oscillations}

\subsubsection{Neutrino Oscillation Theory}

The first theory of neutrino oscillations were proposed by Pontecorvo in 1957 [9, 10]. Because only electron neutrinos were known at that time, the oscillations were not the flavor oscillations we think of today, but rather neutrino to anti-neutrinos oscillations. Neutrino oscillations between flavor eigenstates was developed by Maki, Nakagawa and Sakata [3] in 1962 using the then hypothesized muon flavor to create a two-level system, an idea which was elaborated on by Pontecorvo[4]. Interestingly, these developments in neutrino oscillation theory occurred before the solar neutrino deficit was seen in 1968 . By 1969 Pontecorvo had already published his theory of neutrino oscillations as an explanation of the solar neutrino deficit [11].

Neutrino oscillations arise from a rotation between the neutrino mass and neutrino flavor eigenstates. It is necessary then for the neutrinos to have separate mass eigenstates. Therefore, neutrino oscillations require neutrino mass. As with any rotation, it can be described as a matrix between the two sets of eigenstates. For three generations of leptions, the rotation matrix is $3 \times 3$ and unitary [12]:

$$
\left[\begin{array}{l}
\nu_{e} \\
\nu_{\mu} \\
\nu_{\tau}
\end{array}\right]=M_{P M N S}\left[\begin{array}{l}
\nu_{1} \\
\nu_{2} \\
\nu_{3}
\end{array}\right]=\left[\begin{array}{ccc}
U_{e 1} & U_{e 2} & U_{e 3} \\
U_{\mu 1} & U_{\mu 2} & U_{\mu 3} \\
U_{\tau 1} & U_{\tau 2} & U_{\tau 3}
\end{array}\right]\left[\begin{array}{l}
\nu_{1} \\
\nu_{2} \\
\nu_{3}
\end{array}\right] .
$$

$M_{P M N S}$ is called the Pontecorvo-Maki-Nakagawa-Sakata (PMNS) matrix. $\nu_{1}$, $\nu_{2}$ and $\nu_{3}$ are the three neutrino mass eigenstates. We can easily make the 
parallel to rotations in a 3-dimensional Cartesian space, where the matrix can be decomposed into three separate rotations, each represented by an angle:

$$
M_{P M N S}=\left[\begin{array}{ccc}
1 & 0 & 0 \\
0 & c_{32} & s_{32} \\
0 & -s_{32} & c_{32}
\end{array}\right]\left[\begin{array}{ccc}
c_{31} & 0 & s_{31} e^{i \delta} \\
0 & 1 & 0 \\
-s_{31} e^{i \delta} & 0 & c_{31}
\end{array}\right]\left[\begin{array}{ccc}
c_{21} & s_{21} & 0 \\
-s_{21} & c_{21} & 0 \\
0 & 0 & 1
\end{array}\right]
$$

where $c_{m n}=\cos \left(\theta_{m n}\right), s_{m n}=\sin \left(\theta_{m n}\right)$, and $\delta$ is a CP-violating phase allowed in this formalism. ${ }^{3}$

This matrix can be used to derive the probability that a neutrino changes flavor through oscillations. Consider neutrinos that are produced by the weak decay of pions or kaons. Such neutrinos begin in a given weak flavor eigenstate $(\alpha)$, which is a linear combination of the mass eigenstates:

$$
\left|\nu_{\alpha}\right\rangle=\sum_{i} U_{\alpha i}^{*}\left|\nu_{i}\right\rangle
$$

Each mass eigenstate will evolve in time $\tau$ as

$$
\left|\nu_{i}(t)\right\rangle=e^{-i\left(E_{i} \cdot \tau_{i}-\overrightarrow{p_{i}} \cdot \vec{x}\right)}\left|\nu_{i}(0)\right\rangle
$$

where $\tau$ is the time in the laboratory (earth) frame, L represents the distance traveled, and $v\left(\nu_{i}\right)$ is the velocity of the neutrino. Note that the phase $\phi_{i}(x, t)=E \cdot t-p \cdot x$ is not gauge invariant, but the difference between the phases of any two mass states, $\Delta \phi_{i j}(x, t)=\phi_{i}(x, t)-\phi_{j}(x, t)$, is gauge invariant. The time and distance are related by $\tau=L / v\left(\nu_{i}\right)$ in this frame. Therefore, the phase difference can be calculated as

$$
\begin{aligned}
\Delta \phi_{i j}(L, \tau) & =\phi_{i}(L, \tau)-\phi_{j}(L, \tau) \\
& =\left(E_{i}-E_{j}\right) \cdot \tau-\left(p_{i}-p_{j}\right) \cdot L \\
& =\frac{E_{i}^{2}-E_{j}^{2}}{E_{i}+E_{j}} \cdot \tau-\frac{p_{i}^{2}-p_{j}^{2}}{p_{i}+p_{j}} \cdot L
\end{aligned}
$$

\footnotetext{
${ }^{3}$ This does not include factors due to the possibility of Majorana neutrinos. 'CP-violation' is the violation of a combination charge and parity conservation during the interaction.
} 
Note that either the time $\tau$ or the velocity $v\left(\nu_{i}\right)$ differs for the two mass eigenstates. We approximate that the time difference is equal to some average time difference plus a correction $\delta t$

$$
\tau(i j)=\frac{L}{\bar{v}}+\delta t=L \frac{E_{i}+E_{j}}{p_{i}+p_{j}}+\delta t .
$$

Therefore, the phase difference becomes

$$
\begin{aligned}
\Delta \phi_{i j}(L, \tau) & =\frac{E_{i}^{2}-p_{i}^{2}-E_{j}^{2}+p_{j}^{2}}{p_{i}+p_{j}} \cdot L+\frac{E_{i}^{2}-E_{j}^{2}}{E_{i}+E_{j}} \delta t \\
& \approx \frac{\left(m_{i}^{2}-m_{j}^{2}\right) L}{2 E}=\frac{\Delta m_{i j}^{2} L}{2 E}
\end{aligned}
$$

where the vinal equation assumes the momentums are roughly equal to the energy $\left(p_{i}=p_{j}=E_{i}=E_{j}=E\right)$, and the effect of $\delta t$ is negligable. The derivation shown here is slightly different from the usual derivation of neutrino oscillations. The preference for the above derivation is discussed in detail by Harry Lipkin [13].

The probability a neutrino with energy $\mathrm{E}$ that begins in a state $\alpha$ is in a state $\beta$ after a distance $\mathrm{L}$ is given by

$$
P\left(\nu_{\alpha} \rightarrow \nu_{\beta}\right)=\left|\left\langle\nu_{\beta}\left|\sum_{i}\right| \nu_{i}\right\rangle\left\langle\nu_{i}\left|e^{-i \phi_{i}(L, \tau)}\right| \nu_{i}\right\rangle\left\langle\nu_{i}|| \nu_{\alpha}\right\rangle\right|^{2}
$$

Expanded, this equation contains elements where the phases are added together in the exponent. This introduces phase differences with $\Delta m^{2}$ terms into the probability. The probability becomes.

$$
\begin{aligned}
P\left(\nu_{\alpha} \rightarrow \nu_{\beta}\right)= & \delta_{\alpha \beta}-4 \sum_{i>j} \mathbb{R}\left(U_{\alpha i}^{*} U_{\beta i} U_{\beta j}^{*} U_{\alpha j}\right) \sin ^{2}\left(1.27 \Delta m_{i j}^{2}(L / E)\right) \\
& +2 \sum_{i>j} \mathbb{I}\left(U_{\alpha i}^{*} U_{\alpha i} U_{\alpha j}^{*} U_{\alpha j}\right) \sin \left(2 \times 1.27 \Delta m_{i j}^{2}(L / E)\right)
\end{aligned}
$$


where $\Delta m_{i j}^{2}=m_{i}^{2}-m_{j}^{2}$ is in $e V^{2}$ and $\mathrm{L}$ and $\mathrm{E}$ are given in kilometers $(\mathrm{km})$ and gigaelectron-volts $(\mathrm{GeV})$ respectively. The factors of 1.27 appear from dimensional analysis and inclusion of necessary factors of $\hbar$ and c.

\subsubsection{The Two-Flavor Approximation}

A two-flavor approximation of equation (1.8) is commonly used. As shown in Table 1.1, neutrino oscillation experiments have measured the mass splitting $\Delta m_{32}^{2}$ to be much larger than $\Delta m_{21}^{2}$. In equation (1.8), we see that the mass splitting acts as a frequency component to oscillations in L/E. The smaller mass splitting corresponds with a slower frequency of oscillation in L/E. At small values of L/E (short times for the neutrino) $\sin ^{2}\left(\Delta m_{21}^{2} L / E\right) \approx 0$ - Terms with this mass difference will not contribute much to the overall oscillation probability. $\theta_{31}$ has also been measured to be small (see Table 1.1), and therefore, we may approximate $\sin ^{2}\left(\theta_{31}\right) \approx 0$ and $\cos ^{2}\left(\theta_{31}\right) \approx 1$. Starting from equation (1.8) and using $\sin ^{2}\left(\Delta m_{21}^{2} L / E\right) \approx 0$, we are left with the $(\mathrm{i}=3$, $\mathrm{j}=2)$ and $(\mathrm{i}=3, \mathrm{j}=1)$ terms. For the probability $P\left(\nu_{\mu} \rightarrow \nu_{\mu}\right), \alpha=\beta=2$. We find that

$$
\begin{aligned}
P\left(\nu_{\mu} \rightarrow \nu_{\mu}\right) \approx & 1-4 \times\left[\sin ^{2}\left(\theta_{32}\right) \cos ^{2}\left(\theta_{31}\right) \sin ^{2}\left(1.27 \Delta m_{32}^{2} \frac{L}{E_{\nu}}\right)\right. \\
& \left.+\sin ^{2}\left(\theta_{31}\right) \cos ^{2}\left(\theta_{32}\right) \sin ^{2}\left(1.27 \Delta m_{31}^{2} \frac{L}{E_{\nu}}\right)\right] \\
\approx & 1-\sin ^{2}\left(2 \theta_{32}\right) \sin ^{2}\left(1.27 \Delta m_{32}^{2} \frac{L}{E_{\nu}}\right) .
\end{aligned}
$$

Using this simplified formula, it becomes apparent that the two mixing parameters produce different effects in the neutrino oscillation as a function of energy. The mass splitting, $\Delta m_{32}^{2}$, contributes to the position of the minima, whereas the mixing angle, $\theta_{32}$ contributes to it's depth. Figure 1.1 shows the 


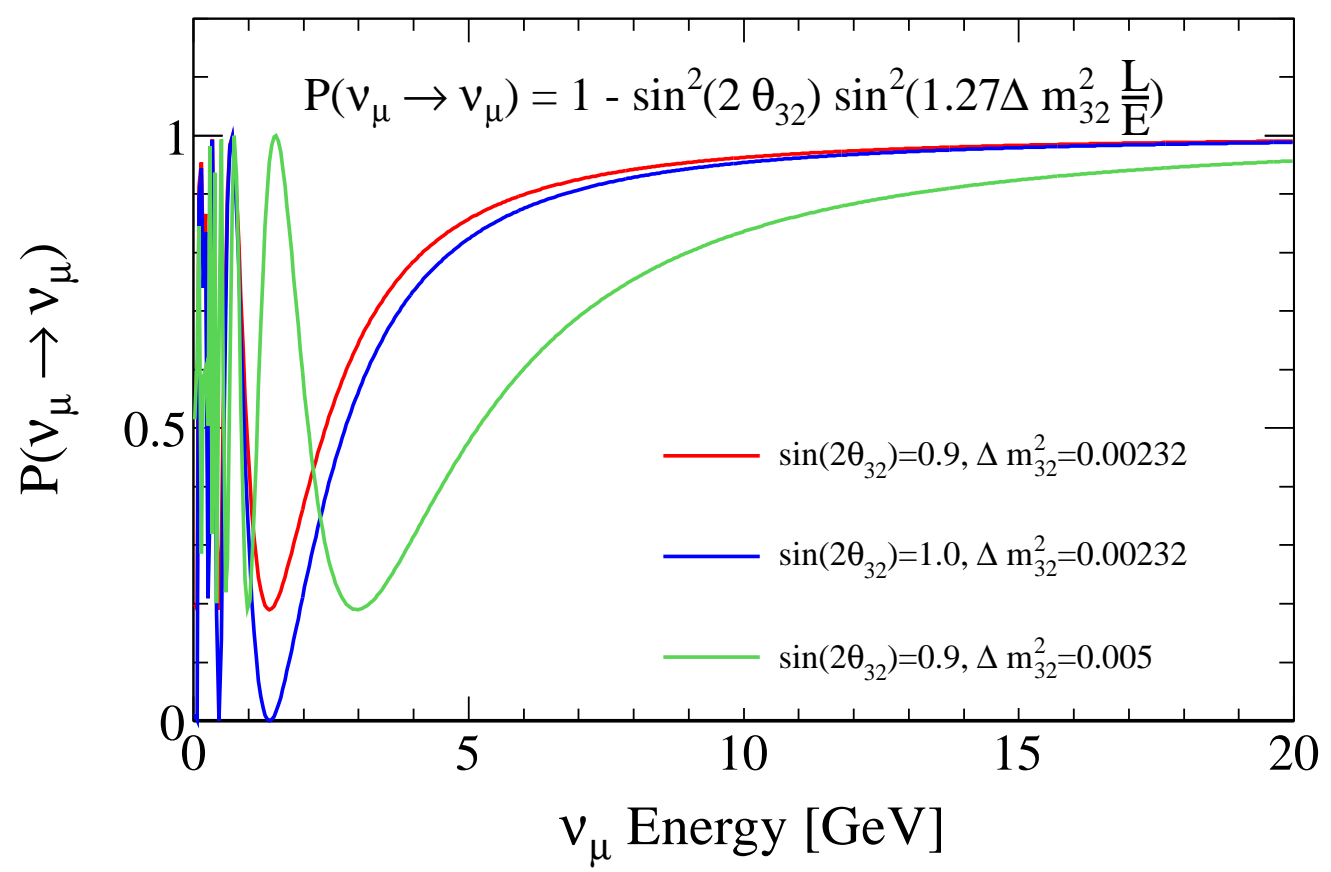

Figure 1.1: The probability of no neutrino oscillation after a distance of $\mathrm{L}=$ $735 \mathrm{~km}$ under the two-neutrino approximation given as a function of neutrino energy in $\mathrm{GeV}$. This is relevant for the MINOS experiment. Example values near the measured value of $\Delta m_{32}^{2}$ and $\sin \left(2 \theta_{32}\right)$ are shown. The mass splitting controls the position of the largest minimum and the mixing angle controls the depth of the minima 
two-neutrino oscillation as a function of energy for a given length "L". The length is fixed to reflect the fixed baseline of most neutrino experiments. In particular, the MINOS experiment has a fixed baseline of $735 \mathrm{~km}$.

In order to measure $\nu_{\mu}$ disappearance, a neutrino experiment must measure the two properties of the neutrinos required by equation (1.8). First, the flavor of the neutrino must be known. That is, it must be known that the neutrinos are $\nu_{\mu} \mathrm{s}$ and not $\nu_{\tau} \mathrm{s}$. The total number of neutrinos summed over all flavors should not show a deficit if neutrinos disappear through oscillations. Secondly, the energy of the neutrino must be measured since because we expect to see an energy-dependent oscillation. Without knowing the neutrino energy, an experiment can only measure the integral deficit through the number of neutrino interactions, but cannot make measurements of the oscillation parameters.

\subsubsection{First Evidence of Neutrino Oscillations}

The first evidence of neutrino oscillations came from the results from the Homestake Experiment, which was designed by Raymond Davis to mea-

sure the number of neutrinos from the Sun produced by nuclear fusion[14]. The primary reactions that produce neutrinos in the Sun detectable by the Homestake experiment are:

$$
\begin{aligned}
& B e_{4}^{7}+e^{-} \rightarrow L i_{3}^{7}+\nu_{e} \\
& B_{5}^{8} \rightarrow B e_{4}^{8}+e^{+}+\nu_{e} .
\end{aligned}
$$

The electron neutrinos produced by these reactions were detected using a tank of perchloroethelene located in the Homestake Mine in South Dakota. Chlorine 
in percholoethelene is changed into radioactive argon through the reaction

$$
\nu_{e}+C l^{37} \rightarrow e^{+}+A r^{37}
$$

Eventually this argon decays into $C l^{37}$ producing a signature electron. The lifetime of this process is on the order of a couple days, which allowed the researchers to collect the argon from the detectors and detect its subsequent decay. Predictions for the electron neutrino rate that was nearly three times larger than the rate observed by the Homestake Experiment [2, 5]. Corrections to neutrino production in the Sun and neutrino detection on earth were unable to reconcile nuclear fusion models and the Homestake data. This discrepancy became known as the "solar neutrino deficit".

The next breakthrough in understanding this deficit did not occur until the Sudbury Neutrino Experiment (SNO). SNO was designed to measure not only electron neutrinos from the Sun, but also muon and tau flavors, which are not expected fusion products. Using a heavy-water Cerenkov detector, SNO was able to measure "charged current" and "neutral current" interactions (see Section 1.3.1) as well as neutrino-electron scattering [15].

The electron neutrino flux was measured through either the charged current interaction, which produces an electron, or through neutrino-electron scattering, which knocks out an atomic electron. By measuring Cerenkov radiation from the outgoing electron, SNO was able to identify electron neutrino charged current and $\nu-e$ scattering interactions. Because the mass of the muon and tau exceeds the typical energy of a neutrino produced by the Sun, ${ }^{4}$

\footnotetext{
${ }^{4} \mathrm{~A}$ typical energy of a solar neutrino is near or less than $14 \mathrm{MeV}$ - the energy from the boron to beryllium transition. The muon mass is $105.7 \mathrm{MeV} / \mathrm{c}^{2}[5]$.
} 
the charged current interaction is exclusive to the electron neutrino. Neutrinoelectron scattering is also dominated by electron neutrino interactions.

The neutral current interaction breaks up the deuteron in heavy water into a proton and neutron. The neutron has a small capture cross-section. During capture, a $6 \mathrm{MeV}$ photon is released. This is distinguishable from the Cerenkov radiation emitted by an electron. This interaction is open to all neutrino flavors, and therefore, SNO was able to measure the total neutrino flux from the Sun.

The SNO experiment confirmed a deficit of electron neutrinos. However, they also found that the total flux from the Sun was in agreement with the solar fusion models. Using data from SNO and the SuperKamiokande (SuperK) experiment, the SNO collaboration was able to demonstrate that neutrino oscillations would provide a satisfactory explanation of the solar neutrino deficit [16].

Observed neutrino deficits are not limited to measurements of neutrinos from the Sun. A muon neutrino deficit has also been measured in accelerator based neutrino beams and from atmospheric sources [17, 18]. In 1988, the Kamiokande experiment observed a 59\% deficit of muon neutrinos from cosmic rays when compared to predictions from Monte Carlo calculations [19]. Atmospheric neutrinos are produced mainly by the reactions

$$
\begin{aligned}
& \pi^{+} \rightarrow \mu^{+}+\nu_{\mu} \\
& \mu^{+} \rightarrow e^{+}+\bar{\nu}_{\mu}+\nu_{e} .
\end{aligned}
$$

These reactions give an expected ratio of 2.0 for $\nu_{\mu} / \nu_{e}$ at the earth. Electron neutrinos are not expected to oscillate during the short journey from the upper atmosphere to the earth (the distance from the sun to the earth is much larger). 
Therefore, by comparing the ratio of $\nu_{\mu}$ to $\nu_{e}$, the Kamiokande experiment was able to measure the deficit of muon neutrinos in a flux independent way.

A follow-on experiment called SuperKamiokande (SuperK) found that the muon neutrino deficit was dependent on the zenith angle -which is inconsistent with flux models for atmospheric neutrinos but consistent with neutrino oscillations[20]. Figure 1.2 shows the ratio of observed to expected number of neutrinos given no oscillations for atmospheric electron and muon neutrinos. So far all measurements of neutrino deficits are consistent with neutrino oscillations and inconsistent with other explanations of neutrino disappearance to high confidence.

\begin{tabular}{|l|l|l|}
\hline Result & Experiment & Notes \\
\hline \hline $0.407 \leq \sin ^{2}\left(\theta_{23}\right) \leq 0.583$ & SuperK $[17]$ & \\
\hline$\Delta m_{23}^{2}=2.33_{-0.08}^{+0.11} 10^{-3} \mathrm{eV}^{2}$ & MINOS $[21]$ & $\Delta m_{31}^{2} \approx \Delta m_{32}^{2}$ \\
\hline $\begin{array}{l}\Delta m_{21}^{2}=8_{-0.4}^{+0.6} 10^{-5} \mathrm{eV}^{2}, \\
\tan ^{2}\left(\theta_{21}\right)=0.45_{-0.07}^{+0.09}\end{array}$ & SNO $[16]$ & KamLAND and SNO data \\
\hline $0.03 \leq \sin ^{2}\left(2 \theta_{13}\right) \leq 0.28$ & T2K[22] & Normal heirarchy shown \\
\hline
\end{tabular}

Table 1.1: A selective (and incomplete) list of neutrino oscillation measurements from SNO, T2K, SuperK and MINOS. The values represented in this table are the single-experiment best limits for their respective variable.

Multiple measurements of neutrino oscillations have been made and the known values are summarized in Table 1.1. The MINOS experiment produces the best measurement of $\Delta m_{32}^{2}$ while the SuperKamiokande experiment (SuperK) contributes the best measurement of $\theta_{32} \cdot{ }^{5}$ SNO, the experiment which confirmed the solar neutrino deficit, continues to probe electron dis-

\footnotetext{
${ }^{5}$ The MINOS measurement presented in Table 1.1 is from the MINOS 2011 publication which uses work derived in this thesis.
} 


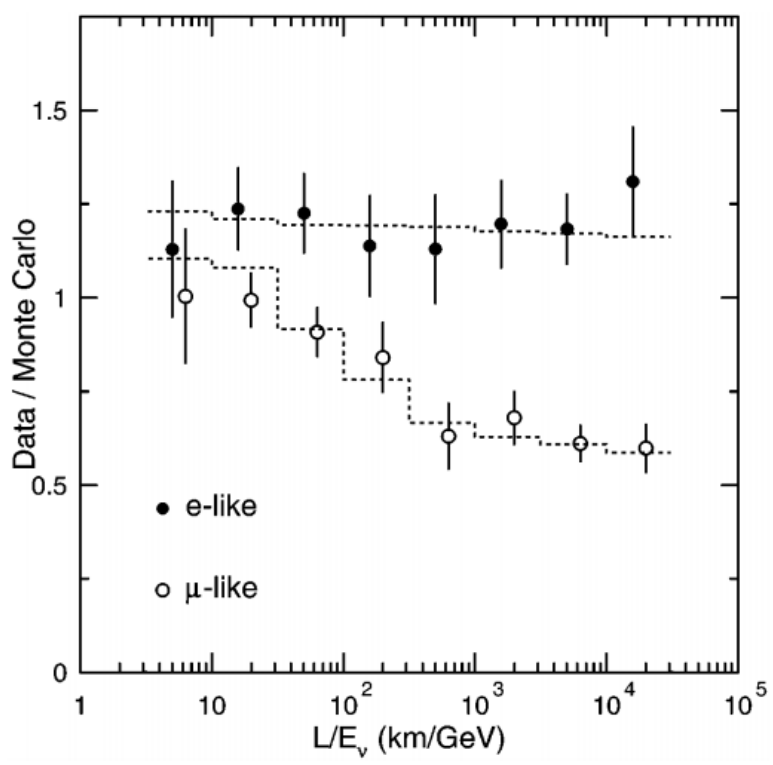

Figure 1.2: The SuperKamiokande result of 1998[20]. Shown is the ratio of observed versus expect number of neutrinos given a no-oscillation hypothesis. The black points are electron neutrino data and Monte Carlo. The open points are muon neutrino data and Monte Carlo. The dashed lines represent the expected shape for $\nu_{\mu} \rightarrow \nu_{\tau}$ oscillations given $\Delta m_{32}^{2}=2.2 \times 10^{-} 3 \mathrm{eV}^{2}$ and $\sin ^{2}\left(2 \theta_{32}\right)=1$. 
appearance from the Sun. SNO provides the best measurements of both $\theta_{21}$ and $\Delta m_{21}^{2}$, which are the largest contributors to the solar neutrino deficit. Note that $\Delta m_{21}^{2} \ll \Delta m_{32}^{2}$, and therefore, it is assumed that $\Delta m_{31}^{2} \approx \Delta m_{32}^{2}{ }^{6}$ T2K (Tokai to Kamiokande) recently measured $\theta_{13}$ for the first time with high enough precision to exclude $\theta_{13}=0$ at above $90 \%$ confidence. Therefore, all the neutrino mixing angles and masses differences have estimates, although they are not all directly measured.

Neutrino oscillations have broad consequences. As the Homestake and SNO experiments demonstrate, neutrino oscillations affect results from astrophysics experiments. However, mixing in the lepton sector mirrors wellestablished mixing in the quark sector and is an easy addition to the current standard model.Indirectly, neutrino oscillations imply neutrino mass. This is at odds with the current understanding of neutrinos as only being left-handed [26]. If neutrinos have mass, right handed neutrinos, which have never been observed, must exist. The upper limit for the neutrino masses is also many orders of magnitude smaller than the lightest charged lepton mass. One possible additional consequence of neutrino oscillations is the existence of normally forbidden decay channels such as $\mu^{-} \rightarrow e^{-}+\gamma$, which have never been measured [27]. ${ }^{7}$ Currently, the standard model does not explains why the neutrino mass is so small.

\footnotetext{
${ }^{6}$ The LSND (Liquid Scintillator Neutrino Experiment) experiment published evidence of a $\Delta m_{31}^{2}$ mass splitting that greatly differed from this assumption using anti-neutrinos [23] and suggested a possible 4 th neutrino mass eigenstate. This was refuted by the MiniBooNE experiment under the assumption of an identical mixing matrix for neutrinos and antineutrinos [24]. However, a controversial new result from MiniBooNE using anti-neutrinos is in agreement with the LSND experiment [25]. Nevertheless, for neutrino mixing, no evidence for a fourth sterile neutrino state exists.

${ }^{7}$ The electron's mass is approximately $0.5109 \mathrm{MeV} / \mathrm{c}^{2}$. Searches for the absolute neutrino mass have set an upper limit of $\sum m_{\nu}<1 \mathrm{eV} / \mathrm{c}^{2}[28]$.
} 
The simplest extension gives the neutrino mass through the usual Higgs mechanism, but states that right-handed neutrinos barely interact. This explains why right-handed neutrinos have never been observed but provides no explanation for why neutrino masses are so small.

Another explanation for neutrino mass is that it arrives through a "seesaw mechanism" [1]. In this theory, a massive sterile neutrino exists $^{8}$ in addition to the Dirac mass terms. The apparent mass of the neutrino is given by $m^{2} / M$, where $\mathrm{M}$ is the mass of the sterile neutrino. The Dirac mass, $\mathrm{m}$, is roughly the size of the other charged lepton masses. An important consequence of this mechanism is that the neutrino is its own anti-particle. Therefore, the mystery of the unobserved right-handed neutrino is solved because right-handed anti-neutrinos have been observed. ${ }^{9}$

Other models have been proposed to explain the neutrino deficits observed. The most popular of these models which do not include oscillations are pure decay and pure decoherence [31, 32]. However, these models are not as successful as neutrino oscillations in describing the energy dependence of the observed deficit [33]. The disfavorment of these other models provides constraints to theories which attempt to predict physics beyond the Standard Model.

Thus, the measurement of neutrino oscillation parameters is important because neutrino oscillations hint at a rich underlying physics yet to be discovered.

\footnotetext{
${ }^{8} \mathrm{~A}$ neutrino with no weak interaction is considered sterile because it only interacts with the Higgs boson.

${ }^{9}$ We find it satisfactory here to overview only the generalities of a type-I see-saw mechanism as an example of a Majorana neutrino explanation for neutrino mass. For a more detailed discussion see the articles by Rodejohann, and Fritzsch and Minkowski [29, 30].
} 


\subsection{Neutrino Interactions}

\subsubsection{Neutrino Scattering in the Standard Model}

Because of the dependencies in equation (1.8), in order to measure neutrino oscillations the energy and the flavor of the neutrino must be known. Understanding neutrino interactions is crucial to reconstructing neutrino events and completing an oscillation analysis.

The simplest model for neutrino scattering is Enrico Fermi's four-point interaction ${ }^{10}$, in which fermions directly interact with each other at a single vertex (see Figure 1.3). In other words, the interaction have zero range. Fermi used this theory to describe charged current interactions, in which the neutrino becomes a charged lepton.

For a neutrino experiment which detects neutrinos from an outside source, the interaction takes the form $a+b \rightarrow c+d$, where a and b represent an incoming neutrino and a stationary "target," and c and d are the final state lepton and recoil target particle. The transition rate for an interaction is given by 'Fermi's Golden Rule:

$$
\begin{aligned}
W & =\frac{2 \pi}{\hbar}\left|M_{i f}\right|^{2} \frac{d n}{d E_{f}} \\
M_{i f} & =\int \phi_{f}^{*} V \phi_{i} d \tau
\end{aligned}
$$

where $\mathrm{W}$ is the transition rate in units of inverse time, $M_{i f}$ is the transition probability from initial state, i, to final state, f, through a perturbation po-

tential $\mathrm{V}$ and $\frac{d n}{d E_{f}}$ is the density of final states. The relationship between the transition rate, $\mathrm{W}$, and the cross-section $\sigma$ is given by

$$
\sigma=\frac{W}{\phi}=\frac{W}{n_{a} v_{i}}
$$

\footnotetext{
${ }^{10}$ Much of the following discussion can be found in Perkin's Introduction to High Energy Physics, Chapters 4 and 7, and Appendix E [34].
} 
where $n_{a}$ represents the density of incident particles (neutrinos) and $v_{i}$ is the relative velocity between interacting particles in the initial state. The variable $\phi=n_{a} v_{i}$ is the flux and represents the number of particles that pass through a plane per cross-sectional area per unit time. The cross-section has units of $1 / m^{2}$ and represents the strength of a particular interaction channel.

Fermi proposed the transition probability $M_{i f}$ was a constant $G_{f}$ multiplied a factor representing the final state spin multiplicity, $P_{i f}$ :

$$
\sigma=\frac{1}{n_{a} v_{i}} W=\frac{2 \pi}{n_{a} v_{i} \hbar} G_{f}^{2}\left|P_{i f}\right|^{2} \frac{d n}{d E_{f}}
$$

The value of $G_{f}$ can be estimated through measurement of the muon decay lifetime. Recent experiments have measured $G_{f} /(\hbar c)^{3}=1.16637(1) 10^{-5} \mathrm{GeV}^{-2}[35]$.

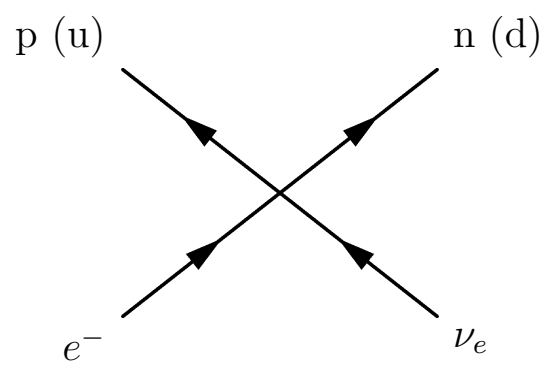

Figure 1.3: Fermi's interaction as a Feynman diagram. The appropriate quarks can be substitute for the proton and neutron in this diagram.

Fermi's theory is remarkably good at explaining neutrino interactions in the low-GeV range. However, one can see that as the $E_{f}$ increases in equation (1.17), the transition rate increases linearly. This is unsustainable, and at high energies, Fermi's theory breaks down. ${ }^{11}$

\footnotetext{
${ }^{11}$ See Perkins, [34], chapters 7 and 9 , for a more detailed explanation.
} 
In this range, a Yukawa potential can be used to describe a timeindependent field of a short range interaction. The Yukawa potential is

$$
U(r)=\frac{g_{0}}{4 \pi r} e^{-r / R}, R=\frac{\hbar}{M_{w} c}
$$

where $\mathrm{R}$ describes the maximum distance traveled by a particle of mass $M_{w}$ bounded by the time particle is allowed to exist by the uncertainty principle $\left(\Delta t \leq \hbar / m c^{2}\right)$. The amplitude of the propagation is given by the Fourier transform of this potential

$$
f\left(q^{2}\right)=g_{0} \int U(r) e^{i \vec{q} \cdot \vec{r}} d V=\frac{g_{0}^{2}}{q^{2}+M_{w}^{2}}
$$

where $M_{w}$ is the mass of the boson propagator, and $\mathrm{q}$ is the three-momentum of the boson propagator. $g_{0}$ is the coupling constant associated with each vertex and the denominator is related to the propagation of the intermediate boson. This boson is the same as what will be called the "W" boson in electroweak theory. The propagation amplitude is related to the strength of the interaction.

In order for the Yukawa boson-mediated interaction to be consistent with Fermi's Four-Point interaction, the propagation amplitude and $G_{f}$ must be the same at the low energy limit, or as $q^{2} / M_{w}^{2} \rightarrow 0$. At this limit the propagation amplitude becomes

$$
G_{f}=\lim _{q^{2} / M_{w}^{2} \rightarrow 0} f\left(q^{2}\right) \approx g_{0}^{2} / M_{w}^{2}
$$

A more complete Vector-Axial derivation would produce the correct relation

$$
\frac{G_{f}}{\sqrt{2}}=\frac{g_{0}^{2}}{8 M_{w}^{2}}
$$

Thus, we find that the strength of the interaction strength is inversely proportional to the square of the mass of the intermediate boson. Replacing 
$G_{f}$ in equation (1.17) with a boson-mediated interaction will reduce the linear divergence in Fermi's theory through the introduction of the $1 / q^{2}$ dependence. At high energies, the cross-section approaches a maximum value. However, the maximum cross-section according to wave-theory (unitarity) is $\sigma_{\max }=\pi \hbar /\left(2 p^{*}\right)$, where $p^{*}$ is the center of mass momentum for a two-body reaction. This cross-section upper limit decreases as $1 / E^{2}$. Further corrections must be made in order to conserve unitarity.

In 1968 Salam and Weinberg proposed a new unified weak and electromagnetic interaction aptly named "electroweak" [6, 7]. This unified interaction solved the unitarity problem by introducing a new boson-mediated interaction. The new neutral current (NC) interaction is mediated by a heavy boson, the $Z^{0}$, but like the photon, does not carry charge, in contrast to the $W^{ \pm}$boson.

In Figure 1.4, two Feynman diagrams representing a charged current (left, $\nu_{l}+d \rightarrow l^{-}+u$ ) and a neutral current event( right, $\nu_{l}+d \rightarrow \nu_{l}+d$ ) are shown. Note that both charged current and neutral current events do not change flavor during the interaction - that is, the lepton flavor $(\mathrm{e}, \mu$, or $\tau)$ is conserved.

For neutral current events, the lepton flavor is carried by a neutrino in the final state. Because the neutrino cross-section is very small, this neutrino is impossible to detect and leaves with a fraction of the event's energy. Therefore, it is difficult to discern the lepton flavor or total energy of a neutral current event. In contrast, the charged leptons resulting from charged current events interact electromagnetically. In particular, muons which are found in the final state of muon neutrino charged current interactions are minimum ionizing. Therefore, charged current events are used as the signal for oscillation experiments because of their identifiable energy and lepton flavor, allowing 
experiments to measure the oscillation parameters in equation (1.8). Neutral current events represent a background to oscillation experiments. This is because neither the incoming neutrino energy or flavor can be determined from the visible final state products of a neutral current event. The selection of charged current events relies heavily on identifying the outgoing lepton. Mis-identifications lead to neutral current backgrounds.
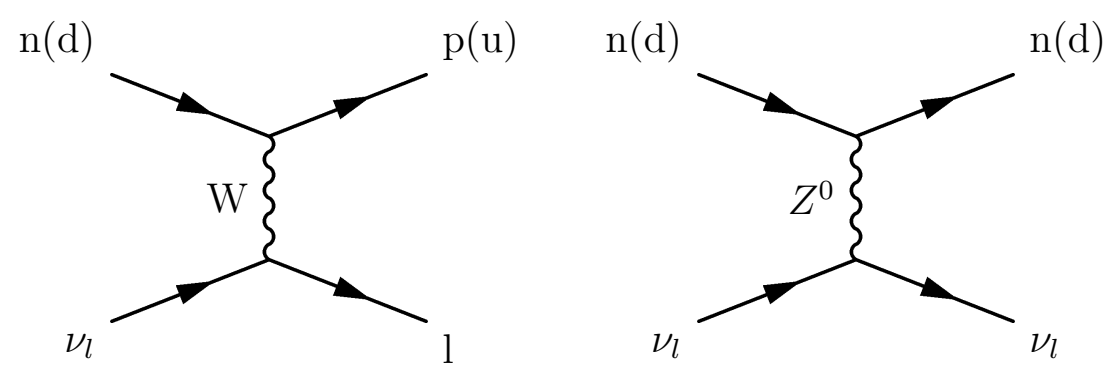

Figure 1.4: Feynman diagrams of a charged current (left) and neutral current (right) neutrino interaction.

\subsubsection{Neutrino Scattering Off Hadrons}

There are three separate contributions to the total neutrino crosssection in the few-GeV neutrino energy region. Each of these exclusive neutrino cross-sections dominates in a particular energy region. The total neutrino cross-section can be decomposed as

$$
\sigma_{\nu(\bar{\nu})}^{C C}=\sigma_{Q E L}+\sigma_{1 \pi}+\sigma_{D I S} .
$$

The three terms are the quasi-elastic cross-section (QEL), the 1- $\pi$ resonant cross-section ( $1 \pi$ or commonly, RES), and the deep inelastic scattering crosssection (DIS). Each of these cross-sections has a different energy dependence. 
The neutrino cross-section is shown as a function of energy in Figure 1.5 from Lipari's paper[36]. ${ }^{12}$

Although we will explicitly be discussing charged current neutrino interactions, neutral current interactions can also be categorized into quasi-elastic, resonant and deep inelastic scattering categories. The obvious difference will be the substitution of the primary outgoing charged lepton with the leptonflavor neutrino. Because neutral current interactions do not transform the nucleus, there are other subtle differences in multiplicity.

The quasi-elastic neutrino cross-section is dominant for energies less than $1 \mathrm{GeV}$. Above $1 \mathrm{GeV}$, a steady decrease in the fraction of quasi-elastic events is seen. In quasi-elastic events, the neutrino effectively interacts with the entire nucleon. The only possible quasi-elastic interactions are

$$
\begin{gathered}
\nu_{l}+n \rightarrow l^{-}+p \\
\bar{\nu}_{l}+p \rightarrow l^{+}+n
\end{gathered}
$$

where $l$ stands for the charged lepton. This is an inverse beta decay reaction and was important for the development of Fermi's theory.

For a free nucleon (e.g. hydrogen) the total energy of the neutrino can be calculated using the energy of the lepton and nucleon, $E_{\nu} \rightarrow E_{n u c}+$ $E_{\nu}$, assuming the target is at rest. The four-momentum transfer, q, is also invariant, and it can be derived that $q^{2}=2 M_{n u c} \nu$ where $\nu$ is the kinetic energy of the nucleus $\nu=E_{n u c}-M_{n u c}$. Unfortunately, in heavier targets the proton can lose energy through nuclear interactions and the precision of this

\footnotetext{
${ }^{12}$ There are multiple in-depth papers that one read for more information, including [3639].
} 


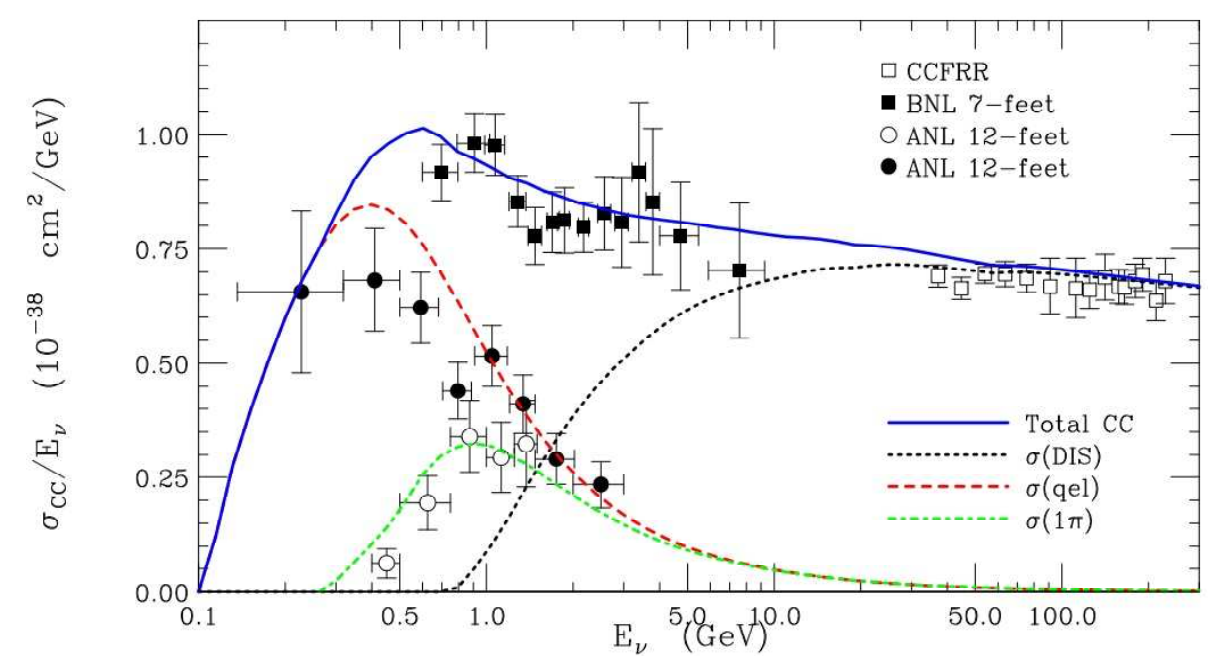

Figure 1.5: The total charged current neutrino cross-section at the few GeV region broken down by its components - quasi-elastic (QEL), resonant (RES) and deep inelastic scattering (DIS). Data from previous neutrino experiments is shown as solid or empty points. These are compared to the closest calculated cross-section line. The calculated cross-section are shown as a solid purple (total cross-section) or dashed (exclusive cross-sections, with the QEL crosssection in red, the RES cross-section in green and the DIS cross-section in black) line. The models used to calculate the curves on this graph are detailed in $[36,39]$. The data shown includes measurements by CCFRR, Brookhaven 7-foot(BNL) and Argonne 12-foot (ANL) experiments 


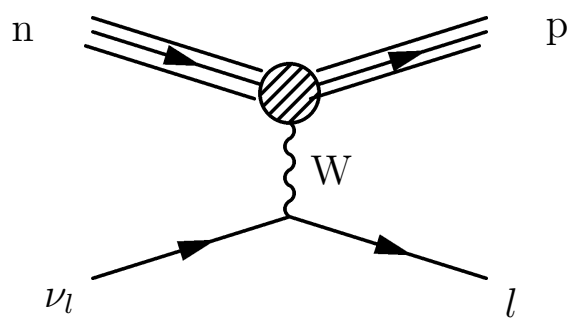

Figure 1.6: A quasi-elastic charged current neutrino event. The signature of a quasi-elastic event is a single recoil proton. Often the proton is not visible in the final state. Such events will appear like a muon with no other energy. In this diagram, the neutrino $\left(\nu_{l}\right)$ enters from the bottom left and transforms into its lepton partner (l). A neutron enters from the upper left corner. The neutron and neutrino exchange a $\mathrm{W}$. The neutron transforms into a proton (p) and exits at the top right.

calculation is lost. For energetic protons this is a fair approximation of the neutrino energy. A theoretical model of the quasi-elastic neutrino cross-section is shown in Figure 1.5 in red.

At energies near 1-3 GeV, the neutrino encounters multiple nucleon resonances. The dominant resonance is the $\Delta(1232)$ resonance. These resonances are the primary contributors to single pion production through the decay of the $\Delta$. The total process is

$$
\begin{aligned}
\nu_{l}+n(p) \rightarrow \Delta^{+}\left(\Delta^{++}\right) & +l \\
& \hookrightarrow p+\pi^{0}\left(\pi^{+}\right) \\
& \hookrightarrow n+\pi^{+} .
\end{aligned}
$$

The relationship between the momentum transfer, $q^{2}$, and the kinetic energy of the hadron system becomes more complicated as some energy goes to creating the $\pi$. If the difference in the mass squared between the initial and 
final states is taken to be $W^{2}-M^{2}$ we find

$$
q^{2}=2 M \nu-W^{2}-M^{2}
$$

Because non-resonant pion production is also possible through final state interactions, generally resonant pion production is limited to the region where invariant mass of the hadron system, $\mathrm{W}$, is less than $2 \mathrm{GeV}$ (This is near the $\Delta$ resonance). Resonant neutrino cross-section data is seen in Figure 1.5 as open circles along a green-dashed line, representing the calculated cross-section. A pseudo-Feynman diagram of a resonant event is shown in Figure 1.7.

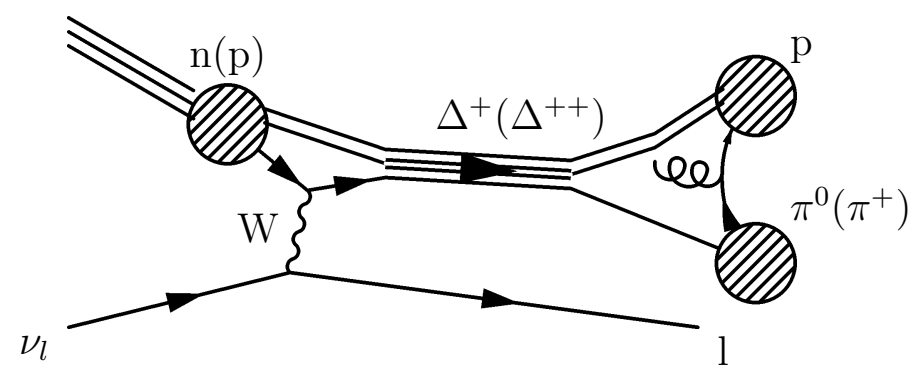

Figure 1.7: A resonant neutrino event is characterized by the decay products of the resonance. For the $\Delta^{+}$resonance, this will include a proton (or undetectable neutron) and a pion in the final state. The neutrino enters at the bottom left of this diagram and transforms into its charged lepton partner, which exists at the bottom right. The neutron(proton) enters from the upper left hand side. Its interaction with the $\mathrm{W}$ boson produces a $\left.\Delta^{+}\left(\Delta^{++}\right)\right)$which decays into a proton and $\pi^{0}\left(\pi^{+}\right)$.

At high energies (above $5 \mathrm{GeV}$ ), deep inelastic scattering (DIS) becomes the dominant form of neutrino interaction. By E $10 \mathrm{GeV}$, DIS accounts for over $50 \%$ of neutrino interactions. Deep inelastic scattering can be subdivided into categories depending on the momentum transfer and hadronic invariant mass. For interactions with $W<2 G e V$ and $q^{2}<1 G e V^{2}$, the alternative term 
"soft DIS" is used. This is to distinguish the transition region from resonance to DIS from the 'pure' DIS region.

For deep inelastic scattering events, the neutrino becomes sensitive to the nucleon's underlying quark structure. The reaction is inelastic because kinetic energy is not conserved, with some energy becoming dedicated to nuclear breakup and the production of final state particles, the process of which is not well understood.

Two important variables for neutrino interactions are the inelasticity, $\mathrm{y}$, and the bjorken scaling value, $\mathrm{x}$, which are defined as

$$
\begin{aligned}
& y=\frac{\nu}{E}=1-\frac{E^{\prime}}{E} \\
& x=\frac{q^{2}}{2 M_{n u c} \nu}
\end{aligned}
$$

where $E^{\prime}$ is the energy of the outgoing lepton. x is the fraction of the maximum $q^{2}$ carried away by the hadron system. For the case of charged current muon neutrino interactions this is $E_{\mu}$. The neutrino cross-section can be written as a function of the energy, $\mathrm{y}, \mathrm{x}$, and $q^{2}$ and under certain conditions, as a function of just the energy, $\mathrm{y}$ and $\mathrm{x}$. A calculated model of the DIS neutrino crosssection is shown in Figure 1.5 as a dashed-black line. Data is shown as open squares. Calculations of the low-energy DIS cross-sections (in the few-GeV region) is needed for the next generation of neutrino oscillation experiments. Furthermore, such events are probes of the parton distributions within the proton and neutron [40].

Note that neutrino cross-sections are generally well known and understood at high energy, where DIS dominates (See Figure 1.5). At low energies, 


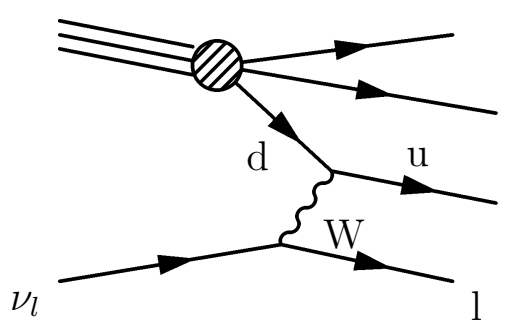

Figure 1.8: A deep inelastic neutrino (DIS) event. The signature of a DIS neutrino interaction is the breakup of the nucleus which results in a hadronic shower. The neutrino enters at the bottom left and transforms into its charged lepton partner, which exits from the bottom right. The incoming hadron's down (d) quark interacts with the $\mathrm{W}$ boson, producing an up quark. The hadron is broken by this interaction.

the number of exclusive neutrino cross-sections which contribute a significant portion of the total (inclusive) neutrino cross-section increases as well as the uncertainty on each cross-section measurement.

\subsubsection{Neutrino Scattering off of Nuclei}

In modern neutrino experiments the target nuclei are often heavy elements including carbon, iron and lead. As the number of nucleons increases, one must consider that the neutrino interaction is taking place in a large nuclear potential. This nuclear potential can effect changes to the apparent neutrino energy or cross-section. Unknown quantities, like the momentum of the interacting quark, impose an uncertainty on the measured neutrino energy that cannot be reduced through experimental techniques. The most important of these final state effects are listed below.

- Pauli suppression: Certain final states are suppressed because they violate the Pauli exclusion principle. For quasi-elastic events at low 
energy, this is a particular problem because the $\nu_{\mu}+n \rightarrow \mu^{-}+p$ reaction is largest at low energies, where the potential for other protons in the nucleus to have the same energy is high. This effectively reduces the cross-section.

- Fermi motion: Nucleons within the nucleus and partons within the nucleon are in motion due to the uncertainty principle. This is usually accounted for using a Fermi gas model or spectral functions [41]. A typical energy for a nucleon is $100-200 \mathrm{MeV} / \mathrm{c}$.

- Structure functions modifications: As the nucleus increases in size, the kinematic distribution of quarks within the nucleus changes [42, 43]. These are especially important for deep inelastic neutrino interactions, where the neutrino is energetic to probe the nuclear structure.
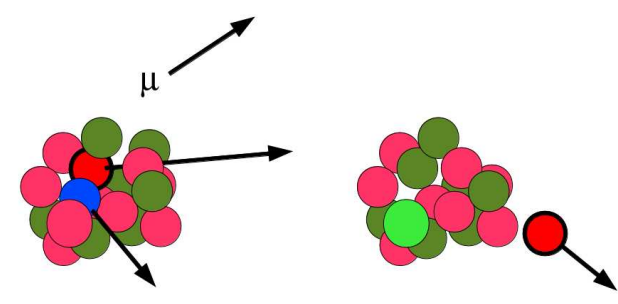

Figure 1.9: Left: A resonant charged current neutrino interaction where the muon leaves. The proton (red) and the pion (blue) must travel through the nucleus. Right: The pion has been absorbed by the nucleus (with the correct charge exchange). The proton leaves the nucleus, but with a different energy and momentum. This event will appear quasi-elastic (1 muon plus 1 proton), and the energy from the recoil is incorrect.

After the initial neutrino interaction, the outgoing hadronic particle is still within the potential of the interacting nucleus. Multiple interactions can 
take place, each of which can modify a particle's original energy, reducing the correlation between the original kinematic state and the kinematic state of the particle (or particles) as it leaves the nucleus and is detected. Prominent final state interactions include elastic scattering, pion absorption, pion production, and charge exchange. Because these interactions occur before the particles can be detected, it is impossible to decouple final state interactions from the original neutrino interaction. Neutrino experiments are dependent on models of final state interactions, which have large uncertainties[44].

The multiplicity of a neutrino event is one way to quantize the final state interactions and nuclear effects in neutrino interactions. These measurements are near impossible without fine-grain detectors like those used in bubble chamber experiments (see Figure 1.10), which can easily distinguish multiple charged hadrons emanating from the vertex. However, using a bubble chamber limits one to perform such studies on low density materials.

Two of the larger systematic errors in the measurement of the neutrino oscillation parameters are related to uncertainties in neutrino scattering crosssections. One systematic error is related to the resolution of the neutrino energy. The reconstructed in part from the resulting muon and in part from the shower of hadronic particles in the reaction $\nu_{\mu}+\mathrm{Fe} \rightarrow \mu^{-}+X$. The resolution on the hadronic energy $X$ for a calorimetric detector such as MINOS is in part limited by our knowledge of the particle species (pion, kaon, proton) in the hadronic shower so that detector pulse height may be converted to energy and is in part limited by nuclear absorption effects which result the particles produced in the hadronic shower being re-absorbed in the nucleus before they can be detected. The second significant uncertainty in the contamination of the charged current event sample by neutral current scatters $\nu_{\mu}+\mathrm{Fe} \rightarrow \nu_{\mu}+X$; 


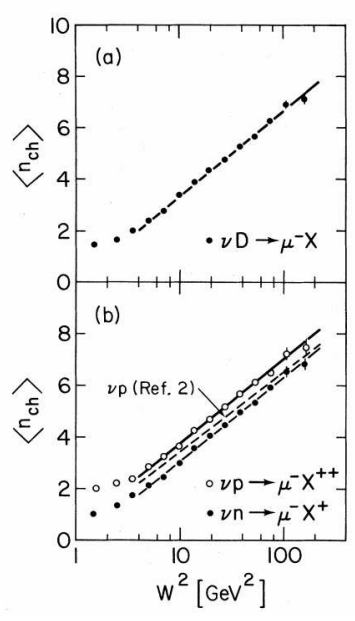

(a) Fermilab 15-foot
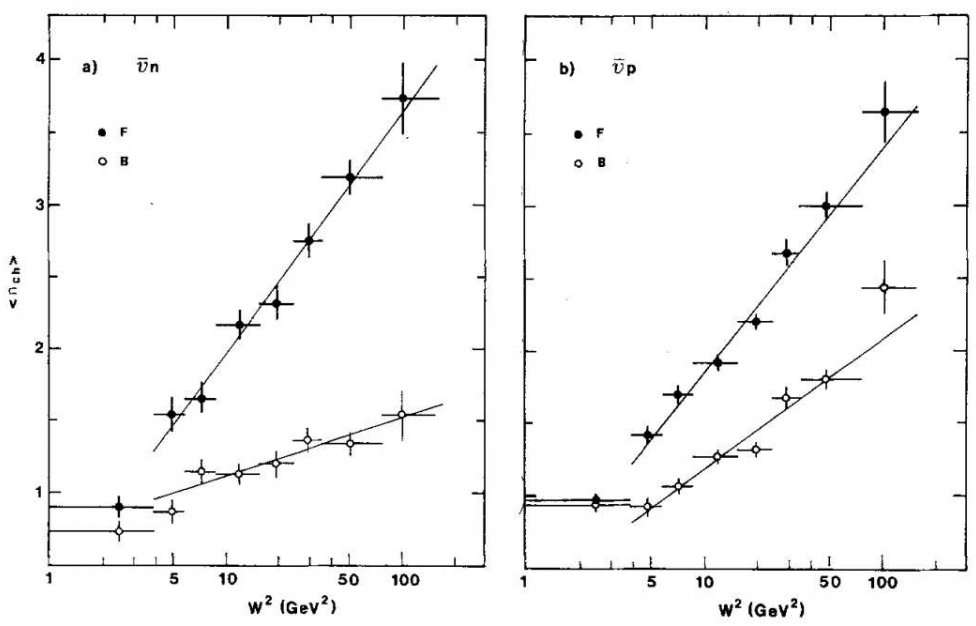

(b) BEBC

Figure 1.10: Left(a):Figure taken from [45] shows the multiplicity measurement of the Fermilab 15-foot bubble chamber. Right: Figure taken from [46] shows the multiplicity measurement for the CERN BEBC experiment. In both cases, the multiplicity is shown as a function of $W^{2}$. The average multiplicity seems to increase with $\ln \left(W^{2}\right)$. For the 15 -foot bubble chamber, the data is split into $\nu_{\mu}+D$ (deuterium) shown at the top or $\nu_{\mu}+p(n)$, shown at the bottom. The solid lines represent linear fits to the data. The dashed line is a fit to both proton and neutron data. The BEBC data (b) is split into $\bar{\nu}+n$ (middle) and $\bar{\nu}+p$ (right). This is further divided into forward multiplicity (solid circles) and backwards multiplicity (open circles). The forward and backwards multiplicities represent the number of particles moving in the forward or backwards directions with respect to the incoming neutrino's direction. 
such events can minic charged currents, but do not leave their entire energy in the detector and furthermore do not properly identify the flavor of the neutrino. The loss of this information tends to dilute the oscillation signal. Our limited knowledge of neutral currentcross-sections makes it difficult to accurately estimate this background.

Both challenges can be addressed with additional measurements of scattering cross-sections, as is proposed in a new experiment at Fermilab, known as MINER $\nu \mathrm{A}$. MINER $\nu \mathrm{A}$ will be situated in the NuMI beam and will have accurate flux measuring instrumentation to normalize cross section measurements. Furthermore, it will be much finer grained than MINOS, permitting measurement of individual particle multiplicities in neutrino scattering events. Lastly, it will will have a variety of nuclear targets ranging from water to lead, permitting the study of nuclear effects in neutrino cross-sections.

The cross section studies at MINER $\nu \mathrm{A}$ will be of interest to other oscillation experiments. The $\mathrm{NO} \nu \mathrm{A}$ experiment, for example, will search for the rare oscillation $\nu_{\mu} \rightarrow \nu_{e}$, requiring that experiment to detect a small signal for electron showers produced in $\nu_{e}+N \rightarrow e+X$ scatters against a background from neutral current scatters producing a neutral pion, such as $\nu_{\mu}+N \rightarrow X+\pi^{0}$. Such single pion production in neutral current events also creates an electromagnetic shower, so the rate for this process must be known accurately. Other experiments, such as water Cherenkov experiments like Super Kamiokande, require accurate knowledge of various processes of charged current scatters $\nu_{\mu}+N \rightarrow \mu^{-} \pi(\pi) X$, which can be a background to the typical process utilized in these experiments, quasielastic $\nu_{\mu}+n \rightarrow \mu^{-}+p$. Quasielastics have a better energy resolution, but the background from $\mathrm{CCn} \pi$ scatters can smear this energy measurement. 
For these reasons, some effort is given in this thesis to the extension of measurement tools for neutrino interactions developed for the MINOS experiment toward use in the MINER $\nu \mathrm{A}$ experiment.

\subsection{An Overview of This Thesis}

The primary analysis of this dissertation is the measurement of $\theta_{32}$ and $\Delta m_{32}^{2}$ by the Main Injector Neutrino Oscillation Search (MINOS) experiment. This was done by measuring the energy dependence of the apparent disappearance of muon neutrinos in flight using two detectors. A crucial component of this analysis is the identification of muons and the estimation of systematic errors due to background neutral current events. The Main Injector ExpeRiment for $\nu-A(\mathrm{MINER} \nu \mathrm{A})$ is dedicated to measuring muon neutrino cross-sections and nuclear effects on a variety of nuclear targets in order to improve measurements by future oscillation experiments. The MINER $\nu \mathrm{A}$ experiment requires sophisticated particle identification, including the identification of muons.

Both the MINOS and MINER $\nu \mathrm{A}$ experiments are located on the NuMI beam at the Fermi National Accelerator Laboratory (FNAL). The NuMI beam is source of directed muon neutrinos. The MINOS experiment has two detectors. The Near detector is located at FNAL, and the Far detector is at the Soudan Mine in Minnesota. The MINER $\nu \mathrm{A}$ detector is placed directly in front of the MINOS Near detector and uses the MINOS Near detector as a back-end spectrometer for high energy muons. The NuMI beamline and the MINOS and MINER $\nu \mathrm{A}$ detectors will be described in Chapter 2 and Chapter 3 respectively.

One of the necessary analysis components for both experiments is the identification of the neutrino flavor. This is synonymous with identifying the 
signature muon in a $\nu_{\mu}$ charged current event. Such muon identification becomes increasingly difficult in a high-density detector like the MINOS detectors at energies below $2 \mathrm{GeV}$. At these energies, the MINOS experiment expects to see the largest minima due to neutrino oscillations (as shown by the blue curve in Figure 1.1). The primary analysis of this thesis is concerned with increasing the number of low-energy muon neutrino events without significantly raising background rates in the MINOS $\nu_{\mu}$ disappearance measurement. This analysis will be discussed in Chapter 4 .

The systematic error of the neutral current background rate can be estimated using the muon-removed charged current events. One can compare the mis-identification rate of shower hits in data and monte carlo simulation. Through this method, we can estimate shower modeling uncertainties and reduce the associated systematic error. This error analysis will be detailed in Chapter 5.

In Chapter 7 the results from the MINOS $\nu_{\mu}$ disappearance analysis will be reported. This analysis used the muon identification improvements described in Chapter 4 and the reduced neutral current background systematic error. Subsequently, the muon identification method used in MINOS was applied to the MINER $\nu \mathrm{A}$ experiment. This analysis and other particle identification techniques used in MINER $\nu \mathrm{A}$ are described in Chapter 9. Particle identification is extremely important to the goals of the MINER $\nu \mathrm{A}$ experiment. The recent progress and difficulties in particle identification in MINER $\nu \mathrm{A}$ will be discussed in Chapter 9 as well. 


\section{Chapter 2}

\section{The NuMI beamline}

The detectors of the MINOS and MINER $\nu \mathrm{A}$ experiments are located on the NuMI (Neutrinos from the Main Injector) beam, which provides a steady source of muon neutrinos. In 2012 the NuMI beam will be upgraded for use by the NuMI Off-axis $\nu_{e}$ Appearance $(\mathrm{NO} \nu \mathrm{A})$ experiment. The production of the NuMI beam begins at the Fermi National Accelerator Laboratory (FNAL or Fermilab) with the collision of $120 \mathrm{GeV}$ protons from the Main Injector (MI) accelerator with a stationary graphite target [47]. The protons arrive in $10 \mu \mathrm{s}$

long pulses which can contain between $10^{11}$ to $10^{14}$ protons. These pulses are known as spills and a spill is extracted roughly every 2.2 seconds.

\subsection{The NuMI Beam Facility}

The components of the NuMI beamline are drawn in succession from left to right in Figure 2.1. This section will describe each of the components of the NuMI beamline in detail. Initially, the extracted protons collide with a stationary target. This target is approximately $95 \mathrm{~cm}$ long, which corresponds to about 2 nuclear interaction lengths for the incoming protons. It is composed of 47 segments which are $6.4 \mathrm{~mm}$ wide, $18 \mathrm{~mm}$ high, and $20 \mathrm{~mm}$ long. The segments made of graphite and are spaced by $0.3 \mathrm{~mm}$ [48]. The proton beam and target are aligned on the axis which points from Fermilab to to the Soudan Underground Laboratory in Northern Minnesota. This includes 
a $58 \mathrm{mrad}$ downward angle, such that the final trajectory of the NuMI beam is through the earth. Colloquially, towards the beginning of the NuMI beam is "upstream" and towards the Soudan Mine is "downstream".

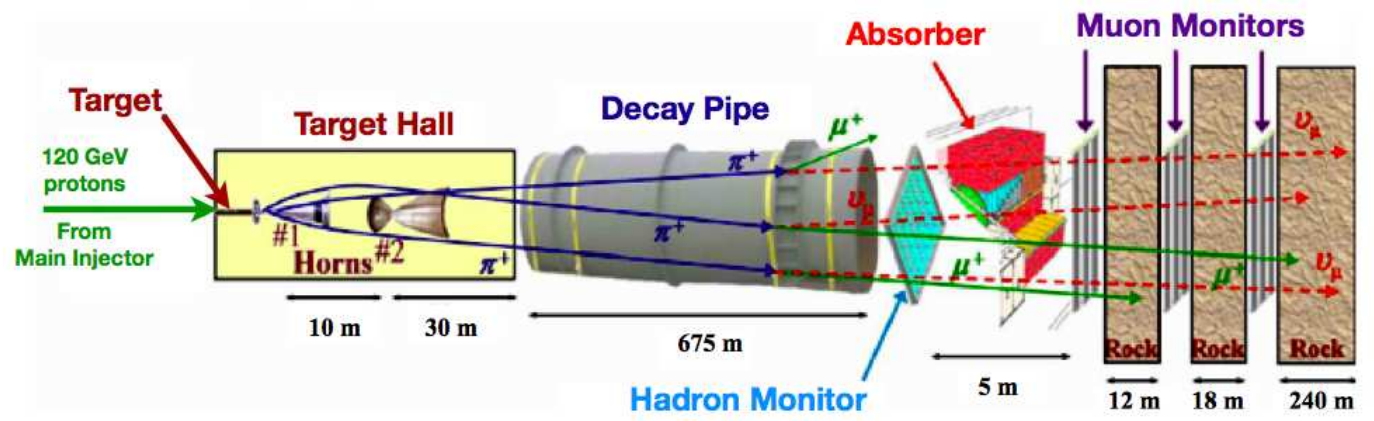

Figure 2.1: A drawing of the NuMI beamiline (not to scale) courtesy of Robert Zwaska [49]. The NuMI beam begins on the left with $120 \mathrm{GeV}$ protons, shown as a green line, centered on a graphite target. Secondaries, shown as blue lines, exit from the target and are focused by the parabolic horns (the parabolic inner conductors are shown, rather than the cylindrical outer conductors). This happens within the Target Hall, which is depicted as a yellow box. The secondaries enter the decay pipe, which is depicted as a grey cylinder. Here, secondaries decay into neutrinos and muons, depicted as red dotted lines and green lines respectively. The Hadron Monitor and Absorber detected and absorb the undecayed secondaries while the muons and neutrinos pass through. The muons are monitored using three muon monitors.

The collision of protons with graphite creates many secondary mesons including pions and kaons. Muon neutrinos are produced in flight [37, 50] by the decay of these mesons, primarily through the following reactions:

$$
\begin{aligned}
\pi^{ \pm} \rightarrow \mu^{ \pm}+\nu_{\mu}\left(\bar{\nu}_{\mu}\right) & B R(99.99 \%) & \tau \approx 26 n s \\
K^{ \pm} \rightarrow \mu^{ \pm}+\nu_{\mu}\left(\bar{\nu}_{\mu}\right) & B R(63.4 \%) & \tau \approx 12 n s \\
K_{L} \rightarrow \pi+\mu+\nu_{\mu} & B R(27.2 \%) & \tau \approx 52 n s .
\end{aligned}
$$


The approximate branching ratios $(\mathrm{BR})$ and lifetimes $(\tau)$ are given next to the reaction. Electron neutrinos are made in smaller quantities from pion decays ( $\left.\pi^{ \pm} \rightarrow e^{ \pm}+\nu_{e}\left(\bar{\nu}_{e}\right)\right)$ and muon decay $\left(\mu^{ \pm} \rightarrow e^{ \pm}+\nu_{e}\left(\bar{\nu}_{e}\right)+\bar{\nu}_{\mu}\left(\nu_{\mu}\right)\right)$ NuMI beam is $92.9 \% \nu_{\mu}, 5.8 \% \bar{\nu}_{\mu}$, and $1.3 \% \nu_{e}$ and $\bar{\nu}_{e}$. The electron and anti-electron neutrinos are produced by the decay of muons through $\mu \rightarrow e+\nu_{\mu}+\nu_{e}$. Pions dominate in both target production and the subsequent decay to neutrinos. Therefore, most neutrinos come from pion decay [48]. There exists a kinematic cutoff energy for the neutrino energy, $p_{\nu}^{\max }=\left(1-m_{\mu}^{2} / M_{\pi / K}^{2}\right) E_{\pi / K}$, where M and $\mathrm{E}$ are the mass and energy of the kaon or pion respectively [50]. Because of this, neutrinos from kaon decay dominate at high energies.

Because the proton-graphite inertial frame is moving in the direction of the incoming proton, the pions and kaons are boosted relativistically in the direction of the original proton beam. However, there are divergences from this direction due to the transverse momentum kicks given to the secondaries from the collision due to Fermil motion of the struck partons in the graphite target. The angle and momentum of secondary particles off the target can be parametrized using $x_{f}=p_{l} / p_{\text {proton }}$ where $p_{l}$ is the momentum of the secondary particle in the direction of the incoming beam (the longitudinal momentum) and $p_{\text {proton }}$ is the incoming momentum of the proton.

Allowing secondary particles to emanate freely from the target will produce a wide, unfocused neutrino beam. Neutrinos cannot be directly focused due to their low interaction rate. In order to create a focused neutrino beam, one must focus the outgoing secondary particles before they decay. This can be achieved by using magnetic focusing objects, called 'horns'. A focusing (or anti-focusing) force can be created with a magnetic field with a direction and strength given by $\vec{F}=q \vec{v} \times \vec{B}$, where $\vec{F}$ is the focusing force, $q$ is the 
charge of the incoming particle, $\vec{v}$ is the velocity, and $\vec{B}$ is the direction of the magnetic field. Given a circular beam directed in the forward $\hat{z}$ direction, and expanding in $\hat{r}$, one would like to apply a focusing $-\hat{r}$ force. Therefore, the magnetic field must be in the azimuthal, $\pm \hat{\phi}$ direction (depending on the charge of the particle). Such a magnetic field is created by currents parallel and anti-parallel to the $\hat{z}$ axis in a cylindrical geometry. In real focusing horns, the current is carried on two concentric, axially symmetric conductors. These are labeled as the "inner" and "outer" conductors. Note that using focusing horns allows one to choose the sign of the focused secondaries (the opposite sign will be anti-focused)[50].

Although the two conductors must be axially symmetric to avoid bending the neutrino beam, the radii of the magnetic horn conductors can depend on the longitudinal direction, z (the name "horn" alludes to the z-dependent cylindrical shape of the conductors). It was found that a parabolic-shaped inner conductor $\left(z=a r^{2}\right)$ with a cylindrical-shaped outer conductor $(r=b)$ provided a focusing that was dependent on the particle energy. The focusing of such a parabolic shape is given as

$$
f=\frac{\pi p}{\mu_{0} a I}
$$

where $f$ is the focusing length, $p$ is the momentum, $\mu_{0}$ is the vacuum permeability, $a$ is the constant of the parabolic shape, and $I$ is the current[50]. A point source with momentum $p$ at a distance $f$ upstream of the "lens" of the parabolic magnetic horn will be focused at infinity- or have a straight trajectory. Thus, assuming the target is a point source, the parabolic magnetic horn allows one to focus a momentum of secondary particle from the target.

Adjoining two parabolic shapes minima to minima increases the strength of the focusing horn. This is the shape of the NuMI magnetic horns, which are 
shown in Figure 2.2. The total focusing length can be estimated by combining the two focal lengths under the thin lens approximation. Multiple magnetic horns aid in focusing particles which were over or under-focused by previous horns. A double horn system is used in the NuMI beamline, as depicted in Figure 2.2. The first horn is $335.43 \mathrm{~cm}$ in length with an outer diameter of $34.93 \mathrm{~cm}$. The smallest radius of the inner conductor is $2.7 \mathrm{~cm}$. The second horn is $363 \mathrm{~cm}$ in length, with a diameter of $79.08 \mathrm{~cm}$. Its smallest radius for the inner detector is $8.80 \mathrm{~cm}[48]$.

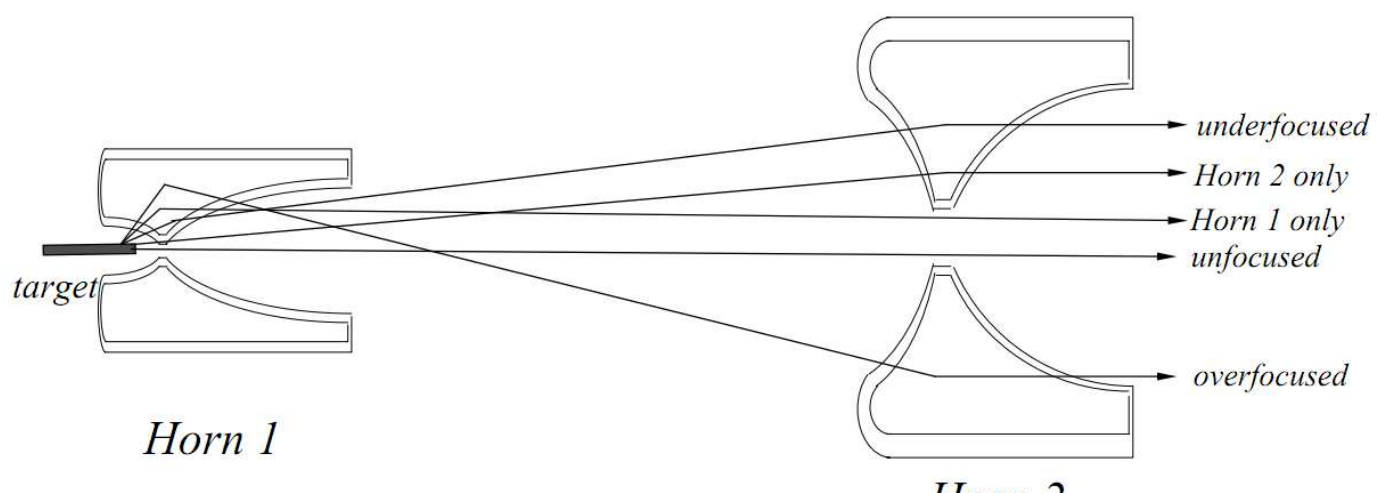

Horn 2

Figure 2.2: Schematic of the NuMI target and focusing horns (not to scale) taken from [50]. The NuMI beam line takes advantage of a two parabolic horn system to help focus secondary particles. Under-focused or over-focused particles get a "second chance" to be focused correctly by the second parabolic horn.

The flux of the NuMI beam at the MINOS Near Detector for the standard NuMI configuration and decomposed by the focusing of the neutrino's parent particle is shown in Figure 2.3. This figure demonstrates the effect of the focusing horns on the composition of the beam. Specifically, it is apparent that the beam peak between 2 and $6 \mathrm{GeV}$ is primarily composed neutrinos whose parent particles were underfocused by the two-horn system. 


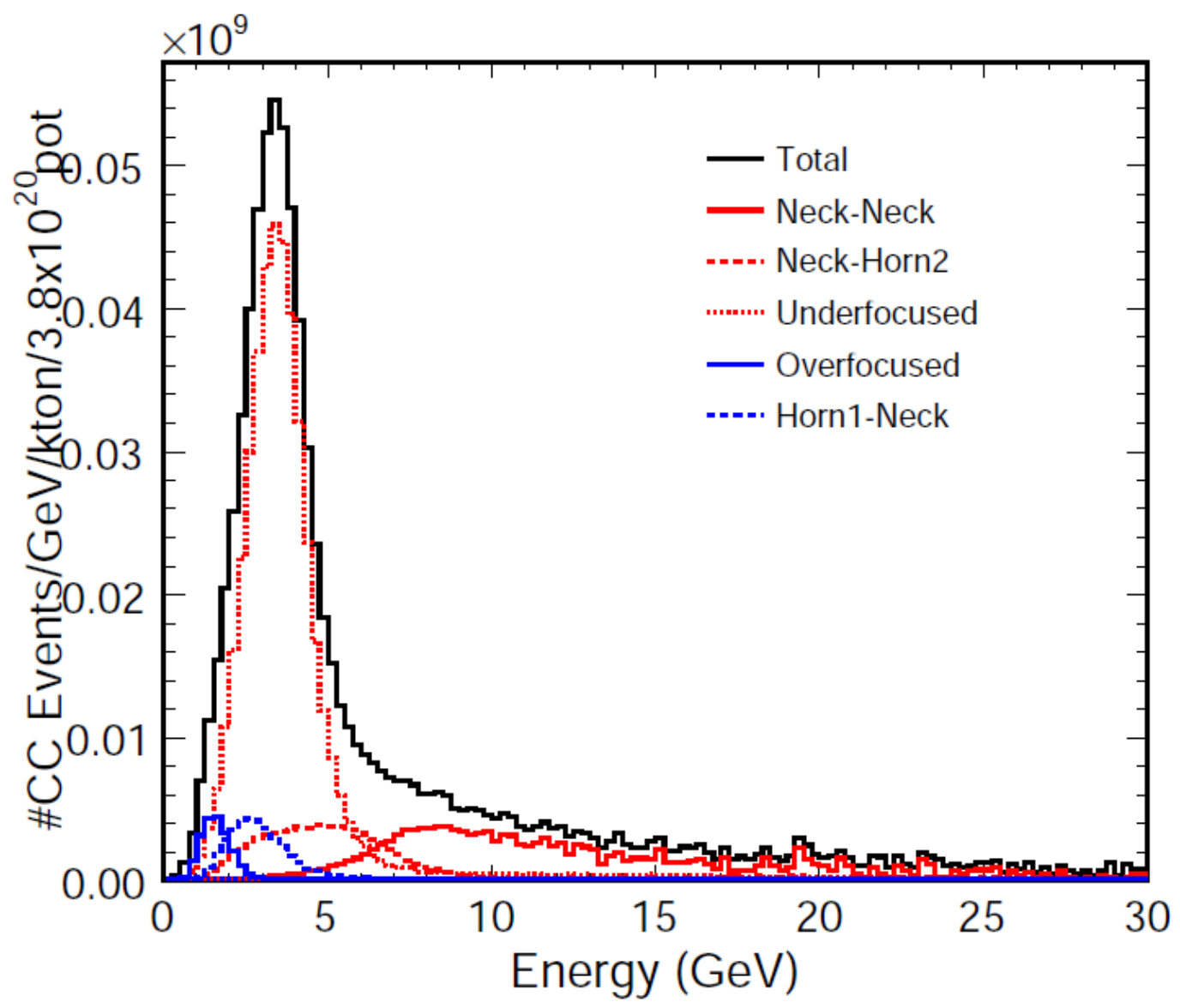

Figure 2.3: The NuMI LE010185N beam flux at the MINOS Near Detector. The total flux is decomposed by which focusing components the neutrino's parent particle interacted with and how focused (underfocused or overfocused) the parent particle is. 
The target and focusing horns are located in the "Target Hall". The NuMI beam was designed with adjustable target and horns because at the onset of the NuMI beam design the oscillation parameters were not well known. Therefore, it was unknown which neutrino energies would be most important for the MINOS oscillation measurement. The target's relative position to the first horn determines the average energy particle focused by the magnetic horns and thus the neutrino energy spectrum. Furthermore, the focusing of the two magnetic horns can be adjusted by changing the magnitude and direction of the current.

Each target position and horn current and position combination defines a "beam configuration". The beam configurations are labeled by the distance of the target from the beginning of the parabolic shape of the first focusing horn in centimeters and the magnitude of the current in the two magnetic horns in kilo-Amperes. This is prefaced by the letters "LE" to denote the "low energy" configuration, where the two horns are separated by a distance of roughly 10 meters. Adjustment of the horn positions (to the high energy, or "HE" configuration) will be done as part of the upgrades for the $\mathrm{NO} \nu \mathrm{A}$ experiment in 2012. Finally, the beam configuration name ends with " $\mathrm{N}$ " or " $\mathrm{R}$ " to specify the current direction in the horns. "N" denotes normal running, where positively charged particles are focused to create a neutrino beam. "R" denotes reverse running, where negatively charged particles are focused to create an anti-neutrino beam. As an example, a common running condition is "LE10185N". This is in the "Low Energy" horn configuration with a 10-cm distance between the target and the first horn. The current is $185 \mathrm{kA}$ in the "normal" current direction.

Figure 2.4 depicts the resulting energy distributions for $\nu_{\mu}$ charged- 
current events at the Near Detector for three beam configurations. The low energy beam configuration in which most of the MINOS charged current analysis data is taken is classified as "LE010185N". This is the lowest energy configuration. A small amount of MINOS data was taken in the pseudo-high energy configuration (labeled pHE or LE250200N).

The target hall ends roughly $30 \mathrm{~m}$ downstream of the second horn and is followed by a cylindrical decay volume. This decay volume lies with its long axis aligned with the NuMI axis. The decay pipe is $675 \mathrm{~m}$ long with an inner radius of roughly $97 \mathrm{~cm}$. This ensures the secondary particles have a low-pressure volume in which to decay rather than interact with matter after being focused. At different times the decay volume has been both evacuated completely or filled with low pressure helium. While traversing this volume, the secondary particles decay, producing both neutrinos and muons. A hadron monitor lies at the end of the decay pipe and measures the number of hadronic particles that remain. Behind this, a hadron absorber stops remnant hadrons that exist at the end of the decay pipe.

Roughly 300 meters of rock separate the hadron absorber and the detector hall, where the MINER $\nu \mathrm{A}$ and MINOS detectors are located. This rock is used to remove muons left during the decay of the secondary particles. In this rock, three muon monitors were installed in separate alcoves. Each alcove is behind a successively larger amounts of rock. The monitors measure the neutrino beam profile by monitoring the muons that are produced along side the neutrinos by the decay of secondary particles. The muon and neutrino kinematics are intimately related through conservation of energy and momentum. The muon monitors provide experiments which use the NuMI beam with an in-situ measurement of the NuMI beam and an independent measure of the 


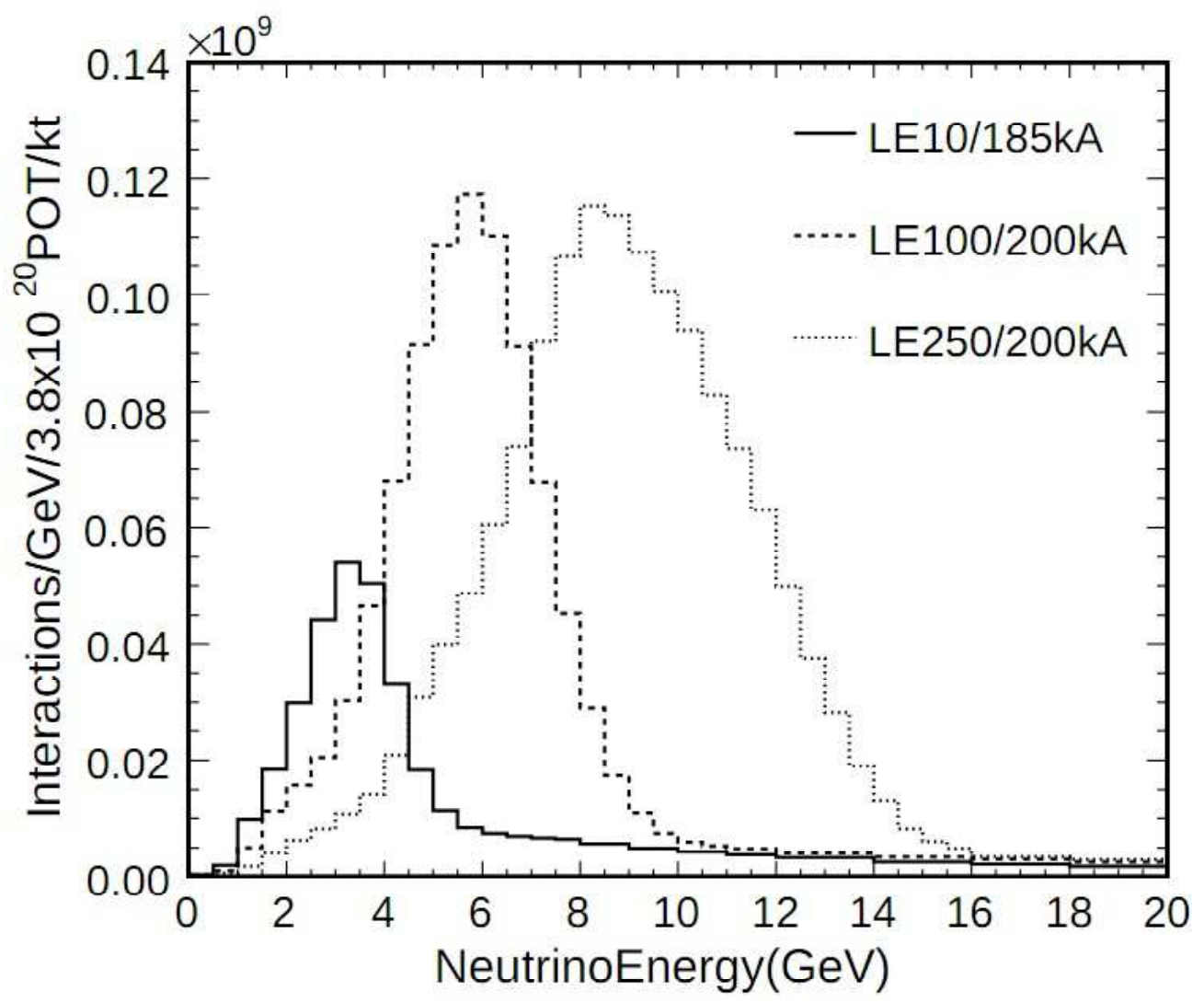

Figure 2.4: The predicted flux at the Near Detector as taken from [33] . The solid black line represents the flux in the "LE10/185kA" mode, where the target is $10 \mathrm{~cm}$ from the parabolic edge of the first horn and the horn current is $185 \mathrm{kA}$. The dotted black line represents the neutrino flux for the "LE100/200kA" mode, where the target is $100 \mathrm{~cm}$ from the parabolic edge of the first horn and the horn current is $200 \mathrm{kA}$. The dashed line represents the neutrino flux for the "LE250/200kA mode, where the target is $250 \mathrm{~cm}$ from the parabolic edge of the first horn and the horn current is $200 \mathrm{kA}$. The flux is given in "Interactions per $\mathrm{GeV}$ per3 $\times 10^{20}$ protons on target per kiloton". 
neutrino flux [51].

A coordinate for the NuMI beam and objects on the NuMI beam can be constructed in which the NuMI axis, pointing from the NuMI Target to the center of the MINOS Far detector (upstream to downstream) is the Z direction. The Y direction is defined as "up", essentially radially out from the earth. This leaves the $\mathrm{X}$ direction pointing parallel to the floor of the detector halls and to the left, assuming one is looking in the positive $\mathrm{Z}$ direction.

\subsubsection{Run Conditions leading up to the MINOS Charged Current Analysis}

The neutrino data gathered and used in the MINOS Charged Current analysis of the atmospheric neutrino parameters is divided into three separate runs. The start of each run represents the installation of a new NuMI target. "Run I" contains data from May 20th 2005 to February 26th 2006 , and June 11th 2006 to August 13th 2006. "Run II" began on September 12 th 2006 and ended on July 17th 2007. During Run II it was discovered that the second target was placed $9 \mathrm{~cm}$, rather than $10 \mathrm{~cm}$ upstream of the first horn's parabolic curve. "Run III" began on November 18th 2007 and ended on June 13th 2009. Run III also contains a modification to the decay pipe volume, which was filled with helium. The effect of the helium was to suppress the focusing peak and increase production in the high energy tail. The total accumulated data is summarized in Table 2.1.

\subsection{Predicting the NuMI Flux at the Near Detector}

The prediction of the NuMI neutrino flux at the MINOS Near Detector and Far Detector requires a Monte Carlo model of the NuMI beam. The Far 


\begin{tabular}{|l|cc|}
\hline Run Configuration & Duration & Total POT $/ 10^{20}$ POT \\
\hline Run I LE & $20 / 5 / 2005-26 / 2 / 2006$ & 1.269 \\
Run I pHE & $11 / 6 / 2006-13 / 8 / 2006$ & 0.153 \\
Run II LE & $12 / 9 / 2006-17 / 7 / 2009$ & 1.943 \\
Run III LE & $18 / 11 / 2007-13 / 6 / 2009$ & 3.881 \\
\hline Total & $20 / 5 / 2005-13 / 6 / 2009$ & 7.246 \\
\hline
\end{tabular}

Table 2.1: The MINOS experiment data accumulation summary.

Detector prediction is found using the measured spectrum in the Near Detector and a Monte Carlo model of the expected differences at the two locations. Therefore, we concentrate first on the predictions of the NuMI beam at the Near Detector. In the next section, we will discuss the extrapolation to the Far Detector.

There have been several Monte Carlo simulations of the NuMI beam. All of these simulations use the FLUKA hadron production model to simulate the production of secondaries off the target [52] and the GEANT physics simulation [53]. The first simulation package to be used by MINOS to estimate the NuMI flux at the Near Detector was called gNuMI. This fortran-based package used a simplified geometry of the NuMI beam line which did not contain the intricate design of the horns or the complete shielding the target hall. This simulation was used in the previous result of the oscillation parameters [33].

Uncertainties in the modeling of the NuMI $\nu_{\mu}$ flux at the Far Detector are one of the dominate sources of systematic error in the measurement of $\Delta m_{32}^{2}$ and $\sin ^{2}\left(2 \theta_{32}\right)$ by the MINOS $\nu_{\mu}$ disappearance analysis. Although the FLUKA cascade model predicts the hadron production relatively well, the flux uncertainty is dominated by uncertainty in the production rates of pions and kaons off the target. Such uncertainties were expected. The MINOS experiment was designed with a Near Detector in order to measure the NuMI beam 
before $\nu_{\mu}$ disappearance has had a chance to develop. The Near Detector can measure the rate of $\nu_{\mu}$ interactions in an environment that is 'functionally similar' to the Far Detector. This neutrino rate is the product of the neutrino flux, the neutrino cross-section, and the efficiencies of the Near Detector. Because the neutrino cross-section is known (at the level shown in Chapter 1, Figure 1.5 and the efficiencies of the Near Detector can be estimated, an estimate of the NuMI flux can be extracted from the Near Detector data.

Each NuMI beam configuration focuses different secondary particles from the target such that the neutrino flux at the Near Detector is vastly different for each beam configuration. By comparing the Near Detector data from different beam configurations with the predictions from the NuMI Monte Carlo simulation, MINOS can accompolish two things. First, MINOS can find the hadron production levels that correspond with the best-fit to Near Detector data using a parametric estimate of the momentum distributions of the produced hadrons. Secondly, MINOS can constrain the uncertainty in hadron production and ,therefore, the NuMI flux [48, 54, 55]. ${ }^{1}$

In Figure 2.5, the ratio of charged pion production is shown for various different simulations, data from the NA49 experiment [56], and the pion production ratio that best describes the Near Detector data (taken from work by Mark Dorman [55]). This method was first developed by Zarko Pavlovic [48]. It is clear that the MINOS and NA49 experiments are in good agreement with each other and in poor agreement with other simulations (including the GEANT simulation used by the MINOS experiment).

\footnotetext{
${ }^{1}$ The muon monitors briefly mentioned previously were designed as a system of independently measuring the NuMI beam. This approach is an important check of the NuMI beam flux and was studied by Laura Loiacono [51].
} 


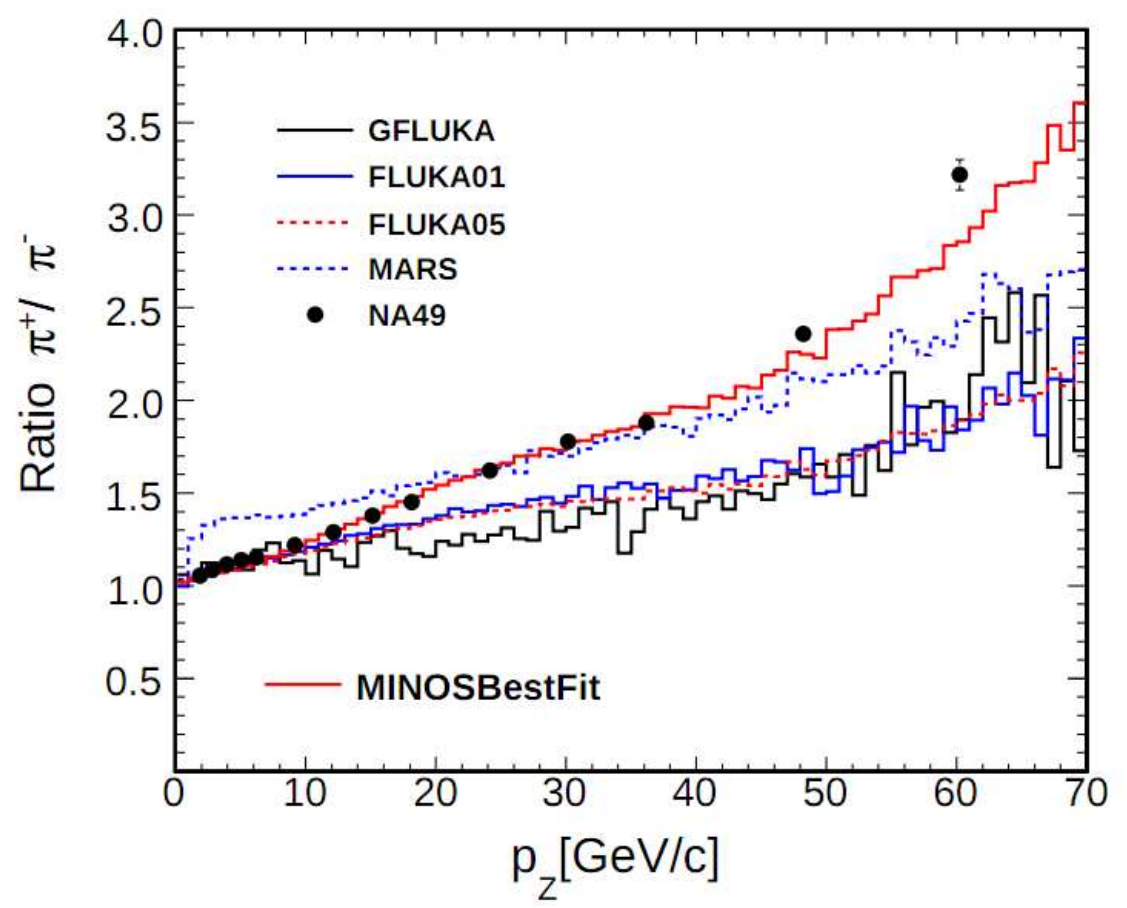

Figure 2.5: The ratio of $\pi^{+}$to $\pi^{-}$production for various different models, the NA49 measurement [56] and the best fit to the MINOS Near Detector data. 
The final beam Monte Carlo utilized to predict the flux at the Far Detector has had its hadron production tuned to reflect the "best-fit to the Near Detector" neutrino rates. These flux-reweightings are crucial for dataMonte Carlo agreement in the Near Detector. The nominal and tuned Monte Carlo predictions of the $\pi^{+}$and $K^{+}$off the target kinematics are compared to data in Figure 2.6. This is an example of the effect of tuning on the Monte Carlo prediction of the data. The tuning proceedure is discussed in detail by Zarko Pavlovic [48]. The Near Detector data, prediction with the nominal Monte Carlo and prediction with the tuned Monte Carlo is shown in Figure 2.7.

\subsection{The MINOS Near-to-Far Extrapolation and the Gen- eral Fitting Method}

Although the Near Detector and Far Detector are functionally similar, their relative angular size as viewed from the NuMI target differ greatly. The Far Detector will only intercept neutrinos whose deviation from the NuMI axis is less than $3 \times 10^{-4}$ degrees whereas the Near Detector can intercept neutrinos which deviate as far as 0.11 degrees from the NuMI axis. Due to the difference in the angular acceptance, the two MINOS detectors are exposed to neutrinos from the decay of secondaries with vastly different kinematic distributions. This is illustrated in Figure 2.8, which shows two pions traveling collinear to the NuMI axis and decaying into neutrinos. The 'soft' pion has a much lower energy than the 'hard' pion. Because the angular distribution of emitted neutrinos falls as $1 / \gamma_{\pi}$, where $\gamma_{\pi}$ is the lorentz boost of the pion, neutrinos from the soft pion are more likely to have a wider angles, which are not covered by the Far Detector. The energy of neutrinos from pion decay $\left(\pi^{+} \rightarrow \mu^{+}+\nu_{\mu}\right)$ 


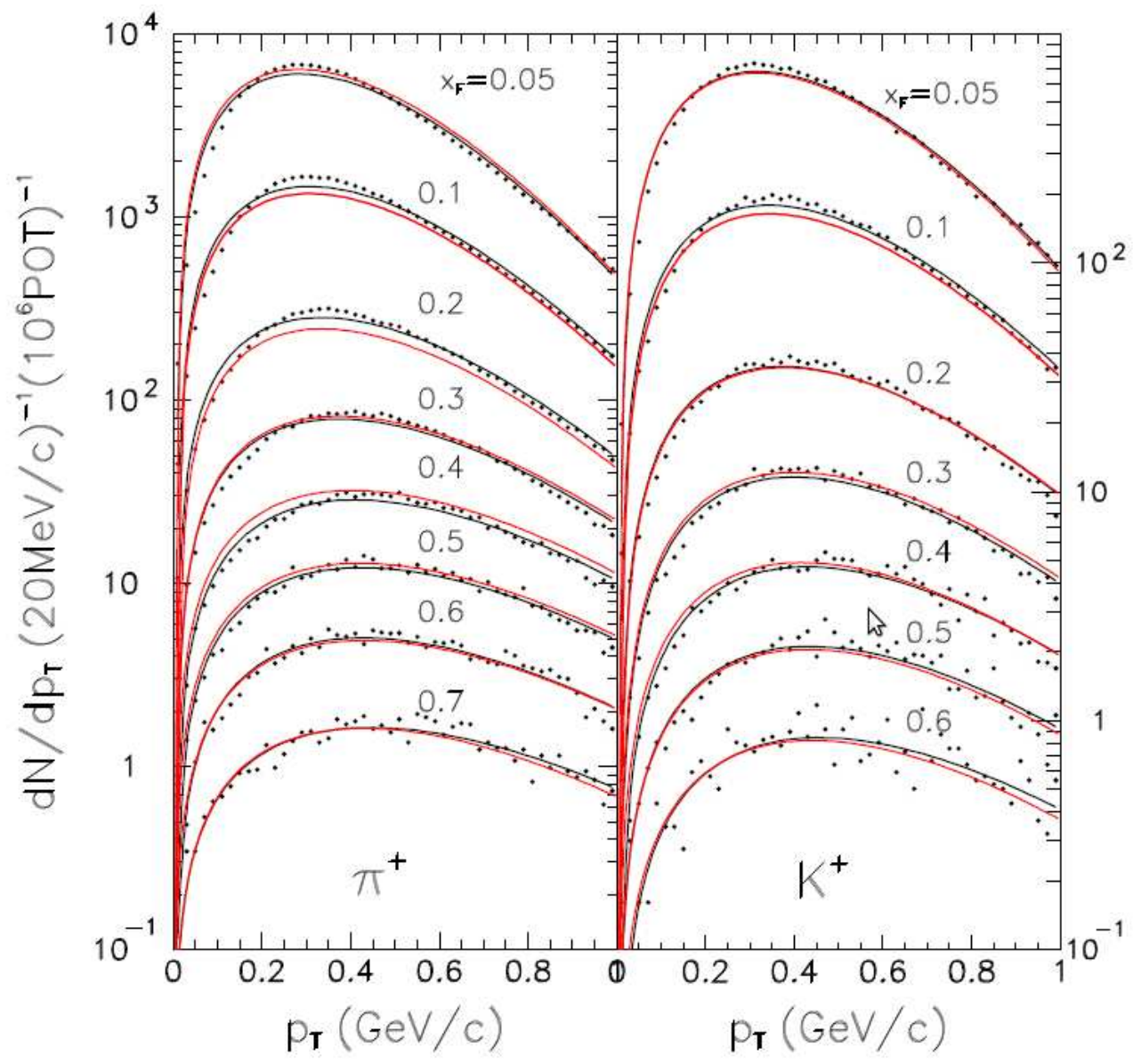

Figure 2.6: The kinematic distributions of $\pi^{+} / K^{+}$production of the NuMI target before(red) and after(black) tuning. This is an example of the effect of the Monte Carlo tuning proceedure. This plot was taken from Zarko Pavlovic[48]. 


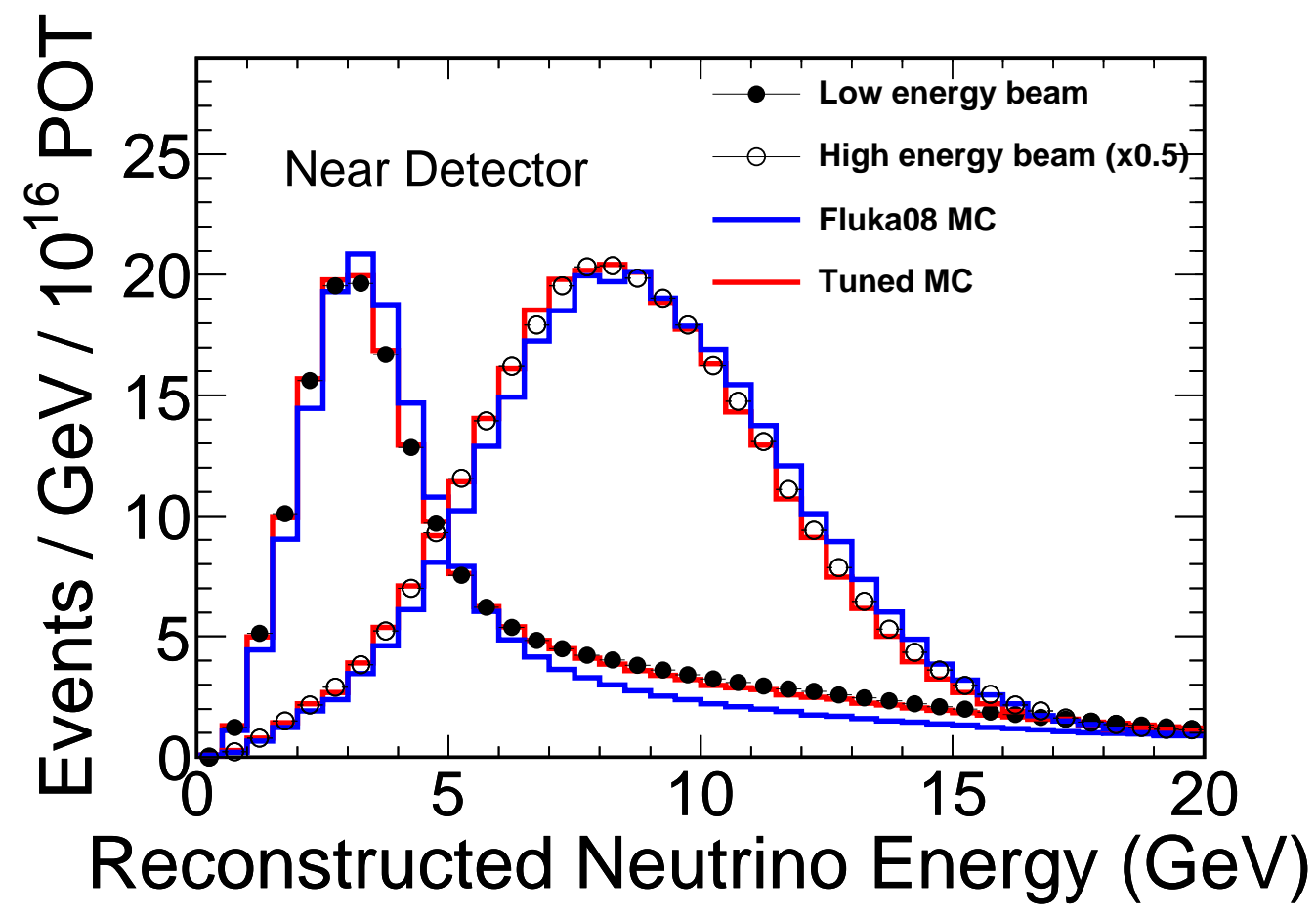

Figure 2.7: The Near Detector neutrino energy spectrum. The black points is the data measured at the Near Detector. The blue histogram is the nominal Monte Carlo using a FLUKA simulation of the target. The red histogram is the tuned Monte Carlo. 
is related to the pion energy by

$$
E_{\nu} \approx \frac{\left(1-\frac{m_{\mu}^{2}}{M^{2}}\right) E}{1+\gamma^{2} \tan ^{2}\left(\theta_{\nu}\right)}
$$

where $m_{\mu}$ and $\mathrm{M}$ are the masses of the muon and pion (or kaon) respectively, $\mathrm{E}$ is the pion (or kaon) energy , $\gamma=E / M$ is the Lorentz boost in the lab frame of the hadron, and $\theta_{\nu}$ is the angle between the neutrino and parent hadron momenta in the lab frame. In addition, soft pions decay more quickly in the NuMI rest frame, producing neutrinos further from the Near Detector than harder pions. For all these reasons, the MINOS Near Detector and Far Detector are sensitive to different ranges of a pion's decay distribution. This leads to differences in the neutrino spectrum.

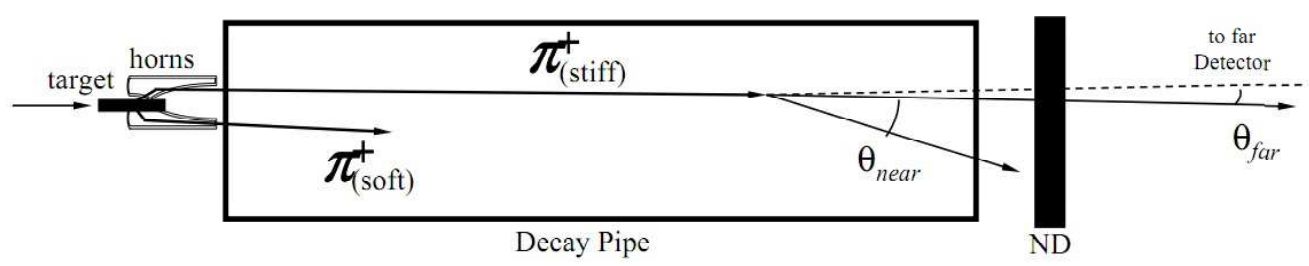

Figure 2.8: The differences between Near Detector and Far Detector spectra are due to a difference in the angular acceptance of the two detectors. Figure taken from [33].

The intricacies of predicting the Far Detector neutrino spectrum with the Near Detector neutrino spectrum must take into account these kinematic differences as well as the loss of events due to the expanding neutrino beam. This 'Near-to-Far' extrapolation of the NuMI beam can be performed using one of two methods. The first method is called the "Far/Near" method and simply predicts the number of events at the Far Detector in a particular energy 
bin, $i$, as:

$$
F_{i}^{\text {Prediction }}=N_{i}^{\text {Data }} \times \frac{F_{i}^{\text {MonteCarlo }}}{N_{i}^{\text {MonteCarlo }}}=F_{i}^{\text {MonteCarlo }} \times \frac{N_{i}^{\text {Data }}}{N_{i}^{\text {MonteCarlo }} .}
$$

This equation can be interpreted in two ways. First, the $F_{i}^{\text {MonteCarlo }} / N_{i}^{\text {MonteCarlo }}$ ratio contains the effects caused by geometric effects like the distance from the NuMI target and the angular acceptance as encoded by the Monte Carlo. Alternatively, it can be interpreted as the Far Detector Monte Carlo being re-weighted by the data-to-Monte Carlo differences seen in the Near Detector to produce a better prediction.

The simplicity of this method is quite alluring; because the Far/Near method does not make distinctions between neutral current and charged current events, it is relatively independent of the neutrino cross and other systematic errors. The pit-fall of this Far/Near extrapolation is its assumption that the Near Detector and Far Detector are absolutely identical, when in reality the two detectors have differences in structure and geometry which could affect the resolution and selection of events. More importantly, the Far/Near method crudely associates a particular energy bin in the Near and Far detectors in spite of the fact that the kinematic distribution of pions that produce neutrinos of a particular energy in the two detectors is different. Since the actual uncertainty in the NuMI beam is hadron production off the target, a better method seeks to recover the hadron spectra and kinematics of the NuMI beam.

The second method of 'Near-to-Far' extrapolation is the 'Beam Matrix' method. This is the main Near-to-Far extrapolation method used by MINOS. In this method, the simple re-weighting of the Near Detector data in equation (2.4) is substituted with a $4^{\text {th }}$ order tensor which sums over the true 
energy at both the Near Detector and the Far Detector as well as the reconstructed energy at the Near Detector. The Beam Matrix method attempts to recover the NuMI pion spectrum from the Near Detector data.

The tensor of the matrix method is composed of multiple matrices, each of which represent a single transformation. Together, these transformations convert the Near Detector data reconstructed energy spectrum into a Far Detector predicted reconstructed energy spectrum. Each of the corrections are derived from Monte Carlo studies. The algorithm to transform a Near Detector to a Far Detector reconstructed energy spectrum is as follows:

1. Tune the Monte Carlo hadron production to best fit the Near Detector data reconstructed energy spectrum.

2. Use this tuned Monte Carlo and knowledge of the efficiency and purity at the Near Detector to estimate of the flux at the Near Detector.

3. Transform this into a flux at the Far Detector (This is the step that uses the Beam Matrix) by 'redecaying' the pions which produced the Near Detector spectrum with the decay kinematics adjusted to produce neutrinos aimed at the Far Detector.

4. Use the predicted flux at the Far Detector and the efficiency and purity of the selection at the Far Detector to estimate the Far Detector event rate.

5. Produce an expected reconstructed neutrino energy spectrum using a True-to-Reco matrix.

The third step uses a probability density matrix, the beam matrix proper, to predict a Far Detector flux using the found true Near Detector 


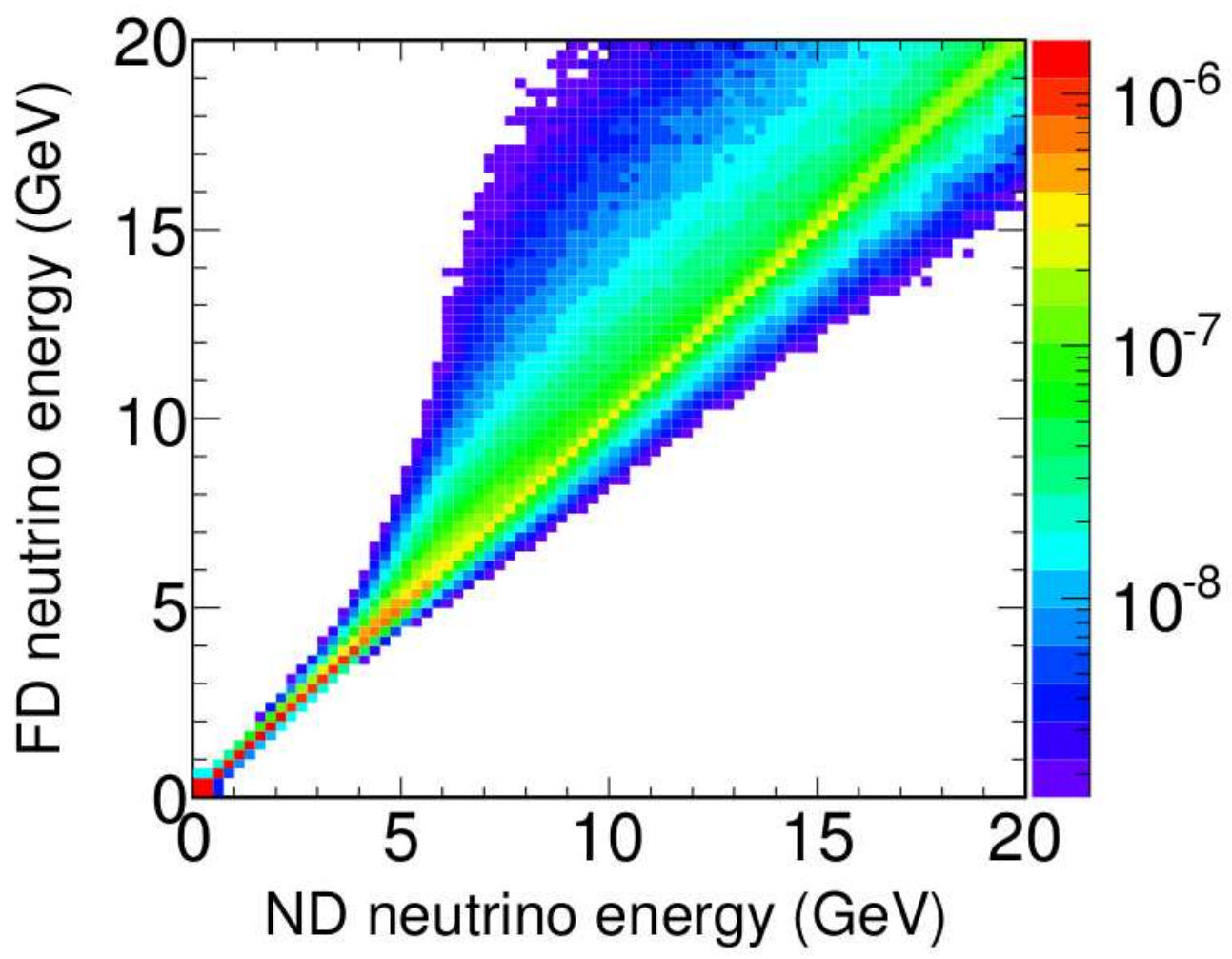

Figure 2.9: The beam matrix for muon neutrinos. This matrix is used to convert a Near Detector $\nu_{\mu}$ true energy spectrum into a Far Detector $\nu_{\mu}$ true energy spectrum 
spectrum. The beam matrix is shown in Figure 2.9. Note the off-diagonal elements to the matrix. This visually demonstrates that a particular Near Detector neutrino energy is associated with a distribution of pion kinematics. These pions can produce a spectrum of energies at the Far Detector. In contrast, the Far/Near method's directly relates Near and Far detector neutrino energy spectra. A matrix for such an assumption would be entirely diagonal, a rough approximation of the matrix derived by the Beam Matrix method. Steps 1 and 2 are mirrored in steps 3 and 4 . These steps take the reconstructed neutrino energy spectrum and convert it into a true neutrino energy spectrum at the Near Detector and vice-versa at the Far Detector. In order to accomplish steps 1,2,4 and 5, the following corrections and transformations must be derived.

- Purity and Efficiency Corrections: The purity and efficiency of the $\nu_{\mu}$ selection must be estimated at both the Near Detector and Far Detector as a function of the reconstructed energy. The purity and efficiency at the Near Detector must be known to tune to the Near Detector Monte Carlo, and the Far Detector purity and efficiency must be estimated to transform the predicted flux at the Far Detector into a reconstructed energy spectrum.

- Reconstructed to true neutrino energy transformation at the Near DetectorIn order to estimate the $\nu_{\mu}$ flux at the Near Detector one most transform the reconstructed energy spectrum into a true energy spectrum. This is matrix is composed using Monte Carlo event true energy and reconstructed energy.

- True to reconstructed neutrino energy transformation at the Far Detector- 
to transform the predicted flux at the Far Detector into a reconstructed neutrino energy spectrum. Again, this matrix is derived by comparing Monte Carlo event true and reconstructed energy.

- Cross-section corrections - To predict the Far Detector event rate from the predicted flux at the Far Detector, one must know the neutrino charged current cross-section. MINOS uses the MODBYRS-4 model, which is written in the NEUGEN simulation [57].

- Detector mass corrections - The Near Detector and Far Detector have significantly different detector masses. The predicted number of events must be calculated per Tonne at the Near Detector and rescaled to the mass of the Far Detector.

This transformative matrix method creates a prediction of the Far Detector reconstructed neutrino energy spectrum in the absence of oscillations. In order to predict the reconstructed neutrino energy spectrum with oscillations at the Far Detector, the true neutrino energy spectrum must be weighted by the probability of oscillations. In the two flavor approximation where only $\nu_{\mu} \rightarrow \nu_{\tau}$ transitions are calculated this is given as

$$
P\left(\nu_{\mu} \rightarrow \nu_{\mu}\right)=1-\sin ^{2}\left(2 \theta_{32}\right) \sin ^{2}\left(1.27 \Delta m_{32}^{2} \frac{L}{E_{\nu}^{\text {true }}}\right) .
$$

The background $\nu_{\tau}$ rate, which increases at a rate proportional to the deficit of $\nu_{\mu}$ must also be added. While more complex, the beam matrix method attempts to correct for various efficiency and contamination issues. In reality, the prediction produced by these two methods differs by less than $5 \%$. The Matrix Method is the main method used to extrapolate the NuMI beam from the Near Detector to the Far Detector and the Far/Near method is used as a 
consistency check. Because the target and running conditions differ for each MINOS run, the Far Detector predicted spectrum is recalculated for each run.

Once a prediction for the Far Detector reconstructed neutrino energy spectrum in the absence of oscillations has been made, the measured Far Detector reconstructed neutrino energy spectrum is compared. The Far Detector reconstructed neutrino energy spectrum is also compared to a prediction with adjustable parameters. The parameters are adjusted such that the loglikelihood is maximized. The log-likelihood function is

$$
\chi^{2}=-2 \ln (\mathcal{L})=2\left[\sum_{j}^{N_{\text {tot }}}\left(N_{j}^{M C}-N_{j}^{\text {Data }}+N_{j}^{\text {Data }}\right) \ln \left(\frac{N_{j}^{\text {Data }}}{N_{j}^{M C}}\right)\right]+\sum_{k}^{N_{\text {systematic }}} \frac{a_{k}^{2}}{2 \sigma_{k}^{2}}
$$

where $a_{k}$ are the different systematic values which are fitted as nuisance parameters, $\sigma_{k}$ are the systematic errors on these values, $N_{\text {tot }}$ is the number of reconstructed bins, and $N_{j}^{M C}$ and $N_{j}^{\text {Data }}$ are the number of events expected by Monte Carlo or seen in data respectively [58]. 


\section{Chapter 3}

\section{The MINOS and MINER $\nu \mathrm{A}$ detectors}

\subsection{Neutrino Detectors on the NuMI beamline}

The MINOS and MINER $\nu \mathrm{A}$ experiments are situated along the NuMI beamline. The MINOS detectors were designed for precision measurements of muon neutrino disapperance through neutrino oscillations, while the MINER $\nu \mathrm{A}$ detector is a high-resolution neutrino interaction detector with multiple target materials. Both detectors are calorimetric sampling detectors which rely on the scintillation light produced by ionizing particles to obtain energy information. These detectors are composed of multiple cross sectional elements stacked along the NuMI axis. By segmenting the scintillator into Z "planes", and each plane into one-dimensional strips, MINOS and MINER $\nu \mathrm{A}$ are able to both estimate the amount and resolve the position of energy deposited by particles in the scintillator.

In this section, we will begin by discussing the physics behind calorimetric detectors. We will then describe in detail the MINOS and MINER $\nu \mathrm{A}$ detectors. 


\subsection{Charged Particle Interactions with Matter}

The MINOS and MINER $\nu$ A detectors rely heavily on charged particle interactions with matter. ${ }^{1}$ In particular, it is expected that particles will lose energy through ionization in the detector. MINOS and MINER $\nu$ A identify particles through the ionization trail left by a particle as it travels. Therefore, understanding ionization is of particular importance. Other particle-detector interactions, such as nuclear scattering and decay often occur as well and aid in particle species identification.

Ionization is the process through which an atom loses an electron. A charged particle traveling through material interacts with the electrons in atoms and can induce ionization. The particle loses energy as it ionizing nearby atoms. The average energy loss of a particle, dE, through a thin material of width, $\mathrm{dX}$, is given by the Bethe-Bloch formula :

$$
\begin{aligned}
-\frac{d E}{d x} & =\frac{4 \pi N_{0} z^{2}}{m_{e} v^{2}}\left(\frac{e^{2}}{4 \pi \epsilon_{0}}\right)^{2} \frac{Z}{A}\left[\frac{1}{2} \ln \left(\frac{2 m_{e} c^{2} \beta^{2} \gamma^{2} T_{\max }}{I^{2}}\right)-\beta^{2}-\frac{\delta(\beta \gamma)}{2}\right] \\
& \approx \frac{4 \pi N_{0} z^{2}}{m_{e} v^{2}}\left(\frac{e^{2}}{4 \pi \epsilon_{0}}\right)^{2} \frac{Z}{A}\left[\ln \left(\frac{2 m_{e} v^{2}}{I\left(1-\beta^{2}\right)}\right)-\beta^{2}\right] .
\end{aligned}
$$

where $\mathrm{m}$ is the mass of the electron, $\mathrm{I}$ is the ionization potential, $\mathrm{z}$ is the charge of the particle, $\mathrm{v}$ is the speed of the particle, $\beta=v / c, N_{0}$ is Avogadro's number (multiplied by the molar mass), $\mathrm{Z}$ is the atomic number and $\mathrm{A}$ is the atomic mass number. $x$ is the path length through the material measured in $\mathrm{g} / \mathrm{cm}^{2}$ (To get the actual path length, one must multiply by the density of the material, $\rho)$. The exact formation has a density effect correction term, $\delta(\beta \gamma)$ and is formulated with $T_{\max } \approx 2 m_{e} c^{2} \beta^{2} \gamma^{2}$. This formula depends only on the particle's energy through $\beta$.

\footnotetext{
${ }^{1}$ This is extensively covered by Leo in [59] and in the "Passage of particles through matter" chapter in $[60]$
} 
For Iron, with a density, $\rho=7.87 \mathrm{~g} / \mathrm{cm}^{3}$, atomic number $\mathrm{Z}=26$, and mass $\mathrm{A}=55.9, m_{e} c^{2}=0.511 \mathrm{MeV}$. This formula becomes

$$
-\frac{d E}{d x} \approx 1.12 M e V / c m \frac{1}{\beta^{2}}\left(\ln \left(3330 \frac{\beta^{2}}{1-\beta^{2}}\right)-\beta^{2}\right) .
$$

The ionization potential can be approximated as $\mathrm{I}=10 \mathrm{Z}$, or found from a table. Figure 3.1 shows the Bethe-Bloch calculated average energy loss for muons, pions and protons in iron. For a typical muon of energy $3 \mathrm{GeV}$, the energy loss through iron is $15 \mathrm{MeV} / \mathrm{cm}$. The dependence of the $d E / d x$ formula on $\beta$ for highly relativistic particles is relatively small, ranging from a minimum of $11 \mathrm{MeV} / \mathrm{cm}$ for a $150 \mathrm{MeV}$ kinetic energy muon to near $20 \mathrm{MeV} / \mathrm{cm}$ at for a $10 \mathrm{GeV}$ muon. Below this, the Bethe-Bloch formula quickly rises as the $1 / \beta^{2}$ factor increases rapidly. Because muons tend to carry a high fraction of the neutrino energy in $\nu+F e \rightarrow \mu^{-}+X$ interactions, they are more likely to be on the slowly-changing high side of the Bethe-Bloch curve.

The Bethe-Bloch formula provides the average energy loss of a particle. This is useful when considering multiple particles traveling through a material. However, the Bethe-Bloch formula has less applicability when considering individual particles traveling through a material. The actual energy loss of a particular particle may widely differ from the mean. The probability distribution of energy loss through a material is given by the Landau distribution, $f(x, \Delta)$, which depends on the thickness of the material, $x$. The Landau distribution peaks at the most probable energy, $\Delta_{p}$, and has an asymmetrically long high-energy tail as seen in Figure 3.2. The width of the Landau function is given as

$$
\begin{aligned}
w & =4 \xi=4 \times(K / 2) \frac{Z}{A} \frac{x}{\beta^{2}} \\
K & \equiv 4 \pi N_{\text {avagadro }} r_{e}^{2} m_{e} c^{2} .
\end{aligned}
$$




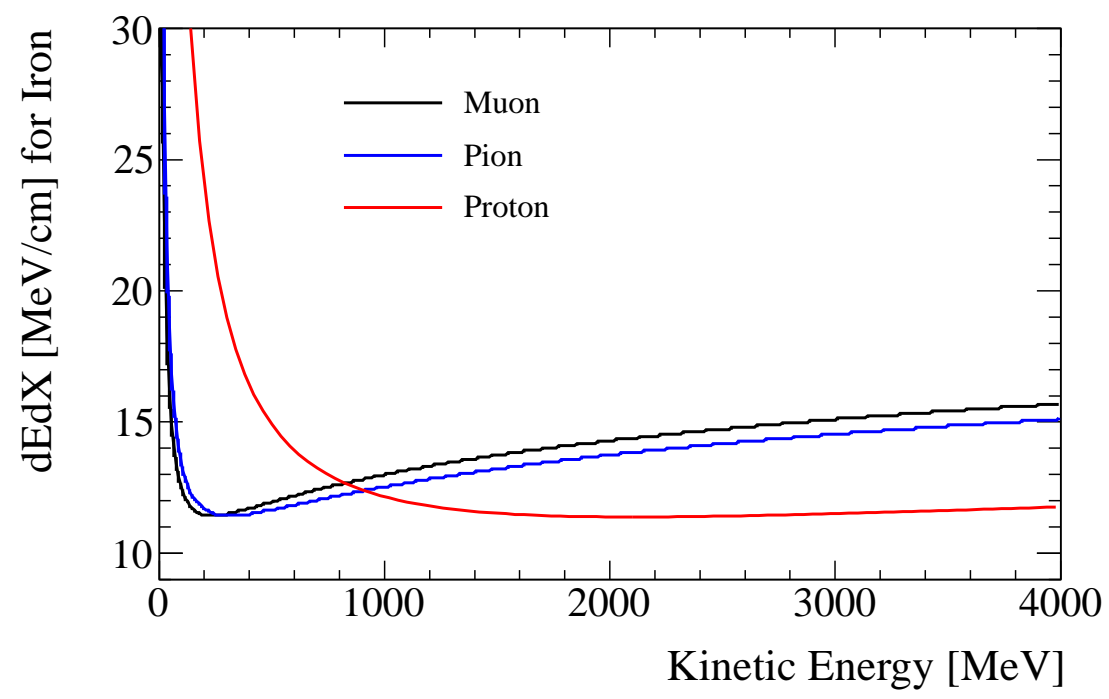

Figure 3.1: The "stopping power" or energy loss per centimeter for particles traveling through iron is plotted versus kinetic energy. The expected stopping power for muons, pions and protons is shown.

The most probable energy loss, $\Delta_{p}$, is given as

$$
\Delta_{p}(x)=\xi\left[\ln \left(\frac{2 m c^{2} \beta^{2} \gamma^{2}}{I}\right)+\ln \left(\frac{\xi}{I}\right)+j-\beta^{2}-\delta(\beta \gamma)\right]
$$

where $\mathrm{j}=0.200$. Note that the most probable energy has a dependence on the thickness of the absorber, $x$, through the variable $\xi . \Delta_{p}$ scales as a $\ln (x)+b$. Thus, the most probable energy reaches a plateau that is not present in the Bethe-Bloch formula. Instead, the Landau distribution at a given energy has a high-energy tail, which predicts that an occasional large energy loss is expected in ionization. ${ }^{2}$

\footnotetext{
${ }^{2}$ As the amount of material traversed increases, an extra term $k=\Delta / E_{\text {max }}$ must be included. This effectively reduces the length of the high energy tail and eventually a gaussian
} 


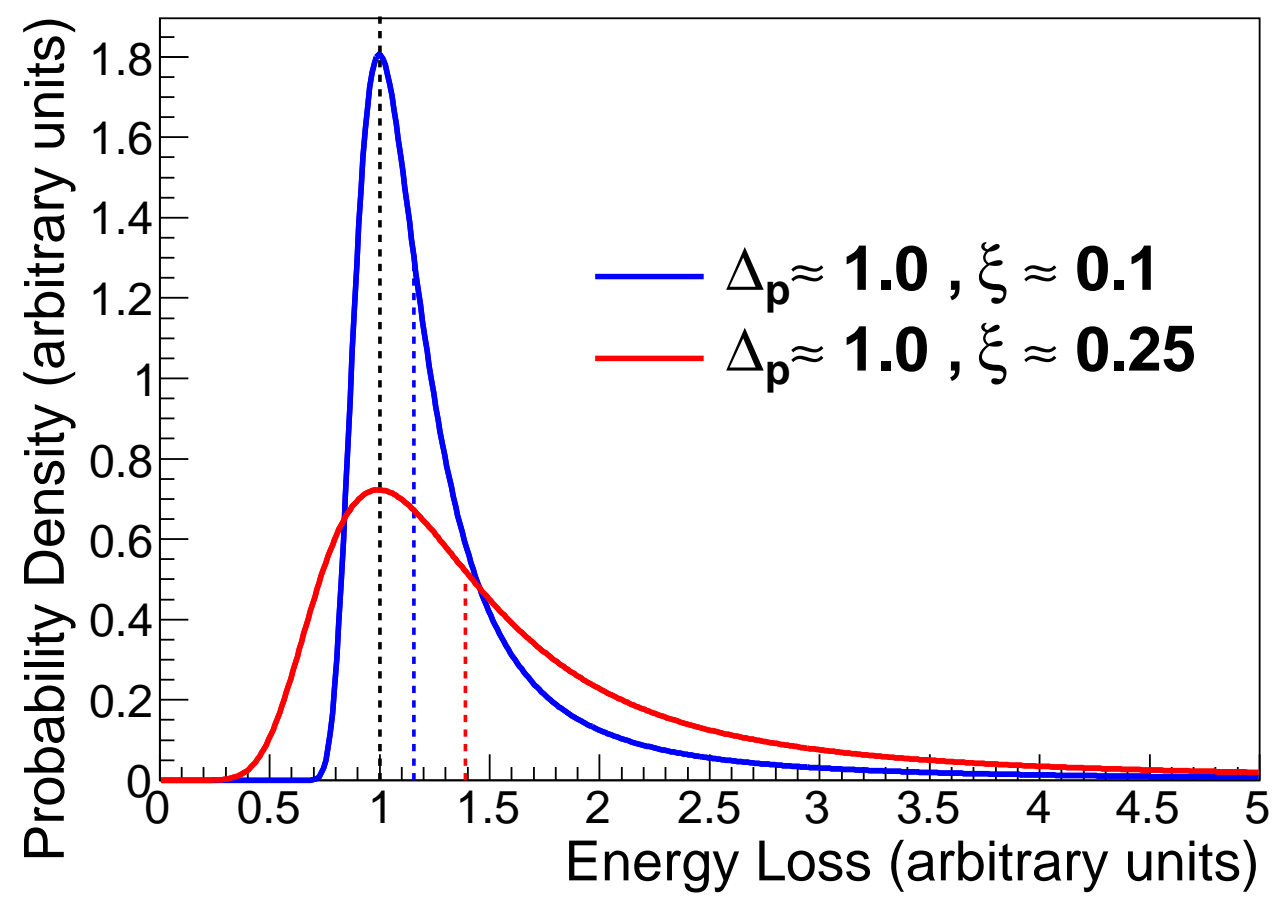

Figure 3.2: A demonstration of the Landau distribution. Both curves are created using the ROOT program approximation of the Landau distribution [61]. These Landau distributions are centered roughly about 1.0 and have a value of $\xi$ of 0.1 (blue) and 0.25 (red). The curves are normalized such that the total probability for each curve is 1.0. The black vertical dashed line is at $x=1.0$ and represents the most probable value, $\Delta_{p}$, for both curves. The blue vertical dashed line is at $x=1.155$ and represents the $50 \%$ probability point (where the probability of having a value less than the point is $50 \%$ ) for the blue curve. The red vertical dashed line is at $x=1.39$ and represents the $50 \%$ probability point for the red curve. The mean energy above the $50 \%$ line for the red curve is roughly 3.25 and 4.7 for the blue curve. The mean energy below these lines is 0.99 for both curves. In addition to having a wider landau shape, hadronic particles will also have non-ionization energy loss, which tends to be high. This will become useful for discriminating muons from other hadronic particles later. 
Both the MINOS and MINER $\nu \mathrm{A}$ detectors rely on scintillation light to detect ionization. The output scintillation light for an ionizing particle is proportional to the energy loss through ionization. This is a common experimental technique [62], and MINOS and MINER $\nu \mathrm{A}$ use similar solid plastic scintillator composed of polystyrene doped with PPO and POPOP fluors[63]. ${ }^{3}$ In Figure 3.3, the Bethe-Bloch energy loss through ionization as measured in polystyrene scintillator used in the MINOS experiment is shown. This demonstrates that scintillator output can be used to measure the energy loss of a muon. The Bethe-bloch curve is used to help determine the conversion from electronic signal to energy measurement [64].

Scattering complicates the reconstruction of particles in neutrino detectors; particles with predictable trajectories are much easier to follow than those with unpredictable direction changes. However, scattering can also be a valuable identifying trait of particles. We can separate these into two scattering processes: multiple elastic and nuclear scattering.

Ionization implies multiple elastic interactions with electrons which ionize atoms along the path of the particle. The large difference in mass between electrons and other particles makes the energy lost to the electron per collision negligible. However, each collision also represents an opportunity for

limit is approached for thick absorbers. This is the Vavilov formulation of energy loss, which is consistent with the Landau formulation for $\mathrm{k}_{\mathfrak{i}} 0.01$. The most probable energy becomes

$$
\Delta_{p}=\left[\ln \left(\frac{2 m c^{2} \beta^{2} \gamma^{2}}{I}\right)+\ln \frac{\xi}{I}+j-\beta^{2}-\delta(\beta \gamma)\right] .
$$

${ }^{3}$ Scintillation light in organic scintillators is the combined light output of fluorescence and phosphorescence processes where a photon is emitted in the decay of a molecule from an excited state to it's ground state. Usually the fast flourescence process dominates and the typical lifetime of this absorption and emission of light is on the order of nanoseconds. 


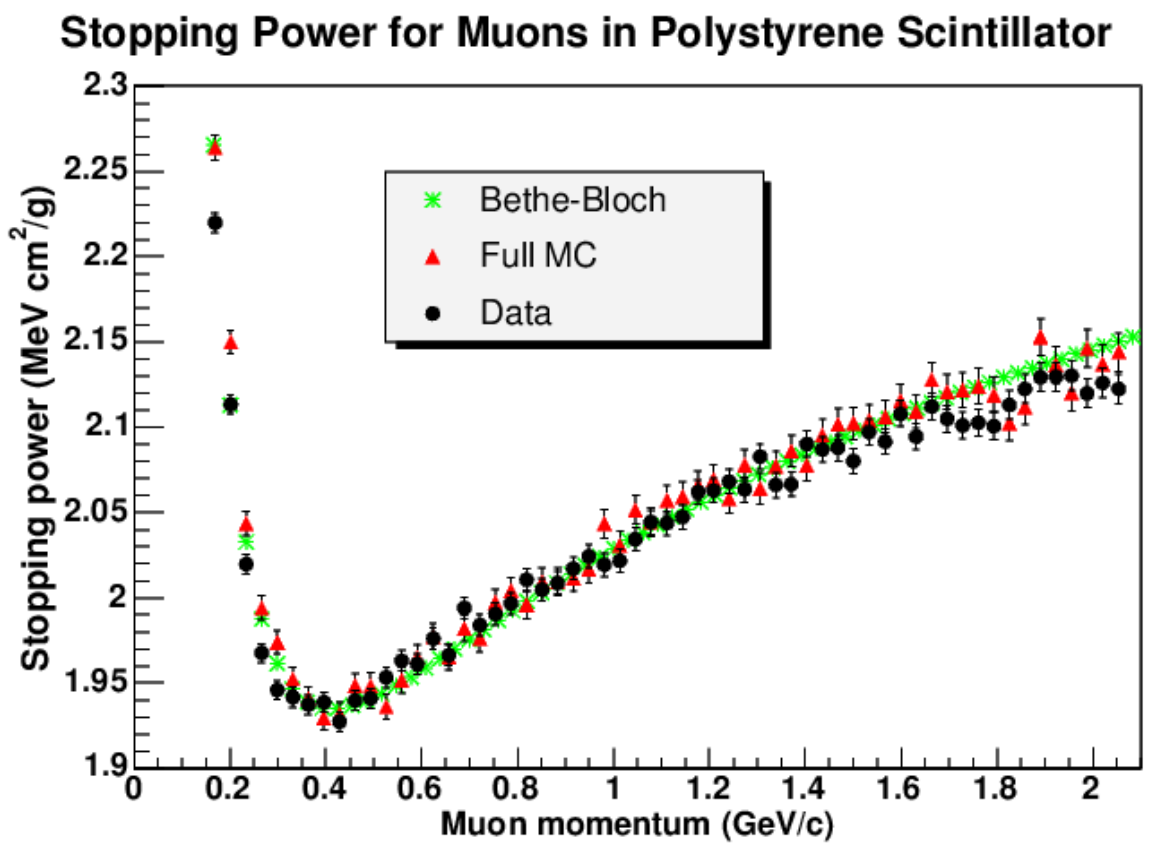

Figure 3.3: A measurement of the energy loss of muons in the MINOS Far detector. The data (shown in black) has been corrected for the length dependence of the fully calibrated detector response. This measure of the BetheBloch curve was an important step in calibrating the MINOS detectors. This figure was taken from [64]. The density of the polysterene was measured to be $1.046 \pm 0.004 \mathrm{~g} / \mathrm{cc}[65]$. 
the momentum direction to change as well. The resulting distribution for this multiple scattering is given by

$$
\begin{aligned}
P(\phi) d \phi & =\frac{2 \phi}{<\phi^{2}>} e^{\frac{-\phi^{2}}{<\phi^{2}}} d \phi \\
<\phi^{2}> & \equiv\left(\frac{z E_{s}}{\gamma \beta^{2} m c^{2}}\right)^{2} \frac{t}{X_{0}} \\
E_{s} & \equiv \sqrt{4 \pi \alpha m_{e} c^{2}} \\
1 / X_{0} & \equiv \frac{4 Z(Z+1) e^{4} N_{0}}{\alpha A m_{e} c^{2}} \ln \left(\frac{183}{Z^{1 / 3}}\right)
\end{aligned}
$$

The width of the distribution is inversely related to the energy and velocity squared.

Nuclear scattering refers to a final state process outside of the incident nucleus of the neutrino event. Such processes are much more likely for pions and protons than for muons. This is related to the final state interactions stated in Chapter 1.

\subsection{The MINOS Detectors}

The MINOS experiment is composed of two detectors. The Near Detector (ND) is located on the Fermilab site, 104 meters underground and 1.04 kilometers downstream from the NuMI target. This detector effectively measures the NuMI beam at time $t_{\nu}=0$, before the neutrinos are predicted to oscillate significantly. The Far Detector (FD) is located in the Soudan Underground Laboratory near Ely, Mn. It is $705 \mathrm{~m}$ underground and $735 \mathrm{~m}$ away from the NuMI target. This distance was selected by the NuMI most peak neutrino energy and the expected value of $\Delta m_{32}^{2}$ during the design of MINOS. 
4 The two detector design of MINOS reduces systematic errors in the oscillation measurement due to flux uncertainties; the neutrino event rate in the Near Detector is used to predict the flux at the Far Detector. Figure 3.4 shows the path traversed by the center of the NuMI beam.

The two MINOS detectors ( Figure 3.5 and Figure 3.6) are functionally similar, meaning that they are composed out of the same materials and basic geometry in order to interact with neutrinos and detect final state particles in a near identical way. This similar design reduces systematic uncertainties due to detector response when comparing data from the two detectors. The detectors are composed of 'planes' made of steel and scintillator. The steel acts as an active target for neutrino interactions and a passive absorber of the final state particles. The scintillator is an active medium, producing light as particles pass through it. This light is collected to obtain calorimetric information. The distance between the planes in both detectors is $\tilde{5} .94$ centimeters from center to center.

Both detectors are placed within a toroidal magnetic field. In the Far Detector, the current carrying coil is placed in the middle of the the detector. In the Near Detector, the coil is placed $4.5 \mathrm{~cm}$ right from the center of the plane (from a NuMI neutrino-eye view). The strength of the magnetic field averages 1.16T in the Near Detector and $1.4 \mathrm{~T}$ in the Far Detector. The magnetic field provides an axially symmetric restoring force to charged particles moving through the detector. This helps prevent particle loss through the sides of the detectors as well as provides a system to identify the muon charge sign. Additionally, the curvature of a muon which traverses the detector in this magnetic

\footnotetext{
${ }^{4}$ At the onset of the design of MINOS, $\Delta m_{32}^{2}$ was thought to be slightly higher than the value known today
} 

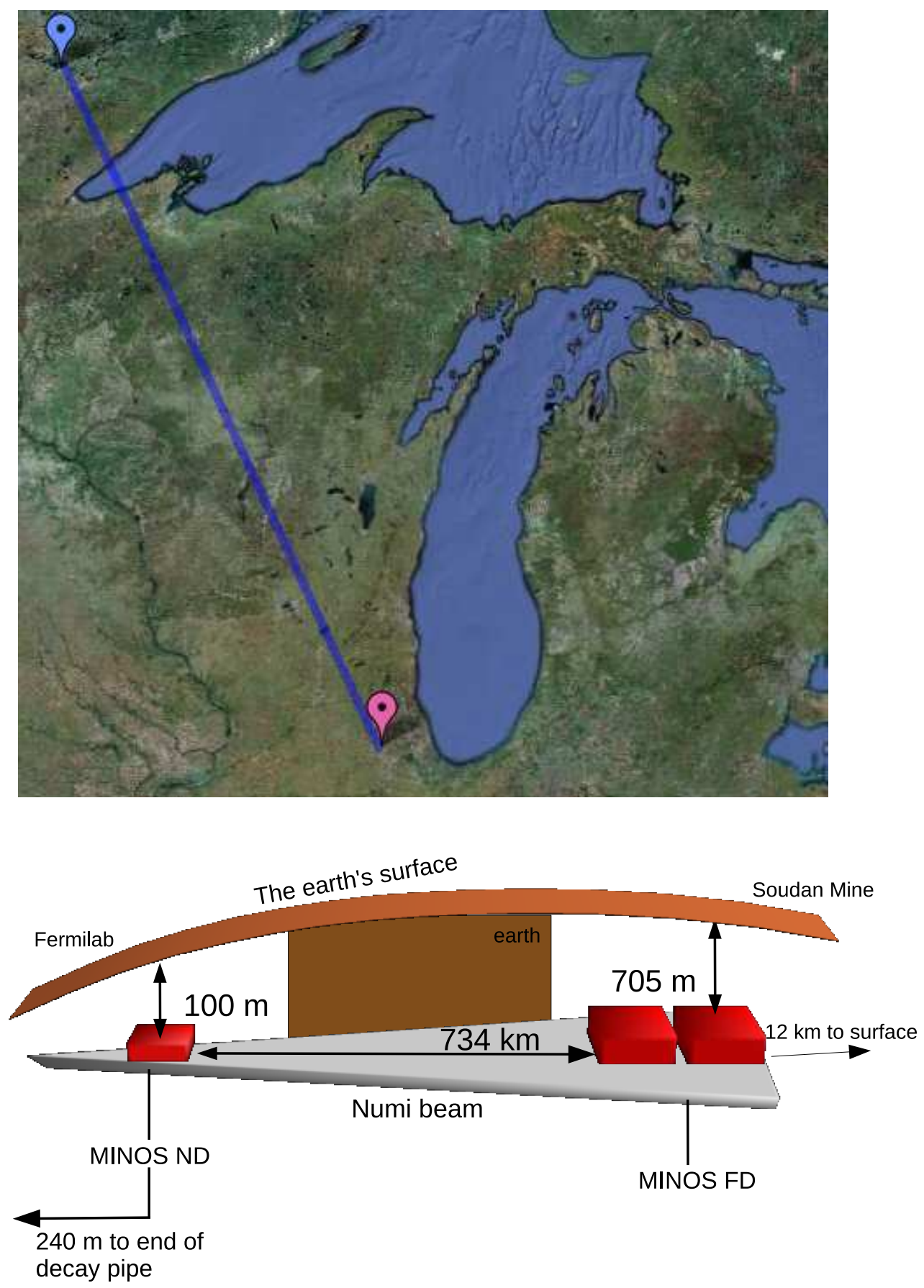

Figure 3.4: The path of the NuMI beam shown in a Google map image (top) [66] and in a cross-sectional diagram (bottom). The blue and purple markers on the satellite image depict where the Far Detector and Near Detector are located. Depictions on the diagram are not to scale. 
field can be used to estimate the muon's momentum. By changing the current direction, the magnetic field will focus particles of opposite electromagnetic charge. Therefore, both "neutrino" ( $\mu^{-}$focusing) and "anti-neutrino" $\left(\mu^{+}\right.$ focusing) magnetic field conditions exist.

As discussed in Chapter 2, the flux at the two detectors differs by orders of magnitude, with the Near Detector observing $10^{6}$ times more events than at the Far Detector per kiloton of detector mass. Therefore, the two detectors have some dissimilarities, especially in size.The Near Detector consists of 282 steel planes. These planes have an irregular $3.8 m \times 4.8 m$ octagonal shape. The first 121 planes of the Near Detector for the "calorimeter" region. This region is designed for detailed information of the interaction vertex. The remaining 161 planes comprise the "spectrometer" region which is designed to measure the momentum of the outgoing muon and act as an absorber. The total mass of the Near Detector is 0.98 ktons.

The Far Detector has 486 steel planes. Each plane has a roughly octagonal shape with an apothem of $4 \mathrm{~m}$ (it stands 8 meters tall and 8 meters wide). The planes are separated into two super-modules with a gap of 1.1 meters separating them. The first super-module is comprised of 249 planes and is 14.78 meters long. The second super-module is comprised of 237 planes and has a total length of 14.10 meters. The total mass of the Far Detector is 5.4 ktons.

Planes which are fitted with scintillator strips are termed "instrumented". This scintillator is in strips, $1 \mathrm{~cm}$ thick and $4.1 \mathrm{~cm}$ wide. In the middle, a wavelength shifting fiber is placed to deliver light to a multi-pixel photomultiplier tube (PMT). In the Far Detector, 484 of the 486 planes are fully instrumented, meaning scintillator covers the entire plane. (The first and last plane in the 
first super-module are uninstrumented) In the Near Detector every fifth plane is fully instrumented, beginning with the first plane. In the calorimeter region of the Near Detector, all other not-fully instrumented planes are partially instrumented. In such planes, only a $3 m \times 3 m$ region of the plane is instrumented and the rest is left bare. In the spectrometer region of the Near Detector, all other planes besides those fully instrumented are left bare. The scintillator strips in MINOS are parallel to one another within a plane. Each instrumented plane has an associated direction defined by the orientation of the scintillator strips. These strip directions are labeled " $\mathrm{U}$ " and "V". They are rotated 45 degrees from the $\mathrm{X}$ and $\mathrm{Y}$ directions. Specifically, $u \equiv(x+y) / \sqrt{2}$ and $v \equiv(y-x) / \sqrt{2}$. The "U" strips are perpendicular to the $\mathrm{U}$ defined view. (and similarly for the "V" view strips) Data from the set of U-positioned strips or $\mathrm{V}$-positioned strips can be viewed versus the longitudinal position (Z-position) of the plane that strip was located in. This creates two "views" of the MINOS data which contain independent spatial information. These are called the U-view and the V-view.

In Figure 3.7 a Monte Carlo simulated event in the MINOS Near Detector is shown. This charged current deep inelastic scattering event has a muon that is well contained in the MINOS detector and curves slightly due to the magnetic field. The hadronic shower is not well resolved spatially, but it's energy is well known through the collected scintillation light. In Figure 3.8 a Monte Carlo simulated charged current quasi-elastic event in the Far Detector is shown. The muon curvature is only apparent in one view. This demonstrates the need for multiple, independent views to collect spatial information. In Figure 3.9 and Figure 3.10 simulated neutral current deep inelastic scattering events are shown for Near Detector and Far Detector. Note the lack of 


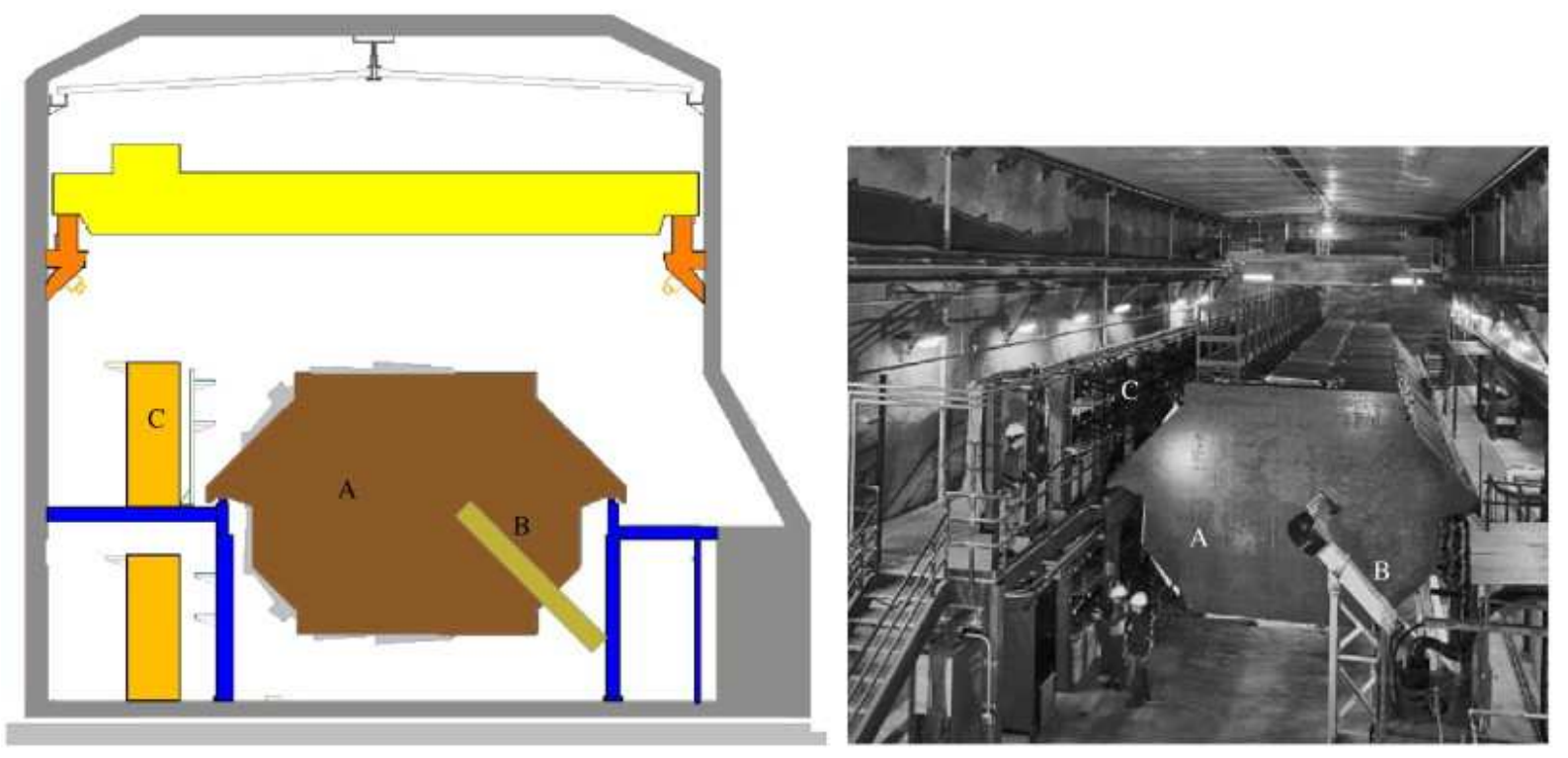

(a) The MINOS Near Detector- cross sectional view diagram(left) and picture(right). Identified are the detector $(\mathrm{A})$, the electronic racks $(\mathrm{B})$ and the current coil $(\mathrm{C})$

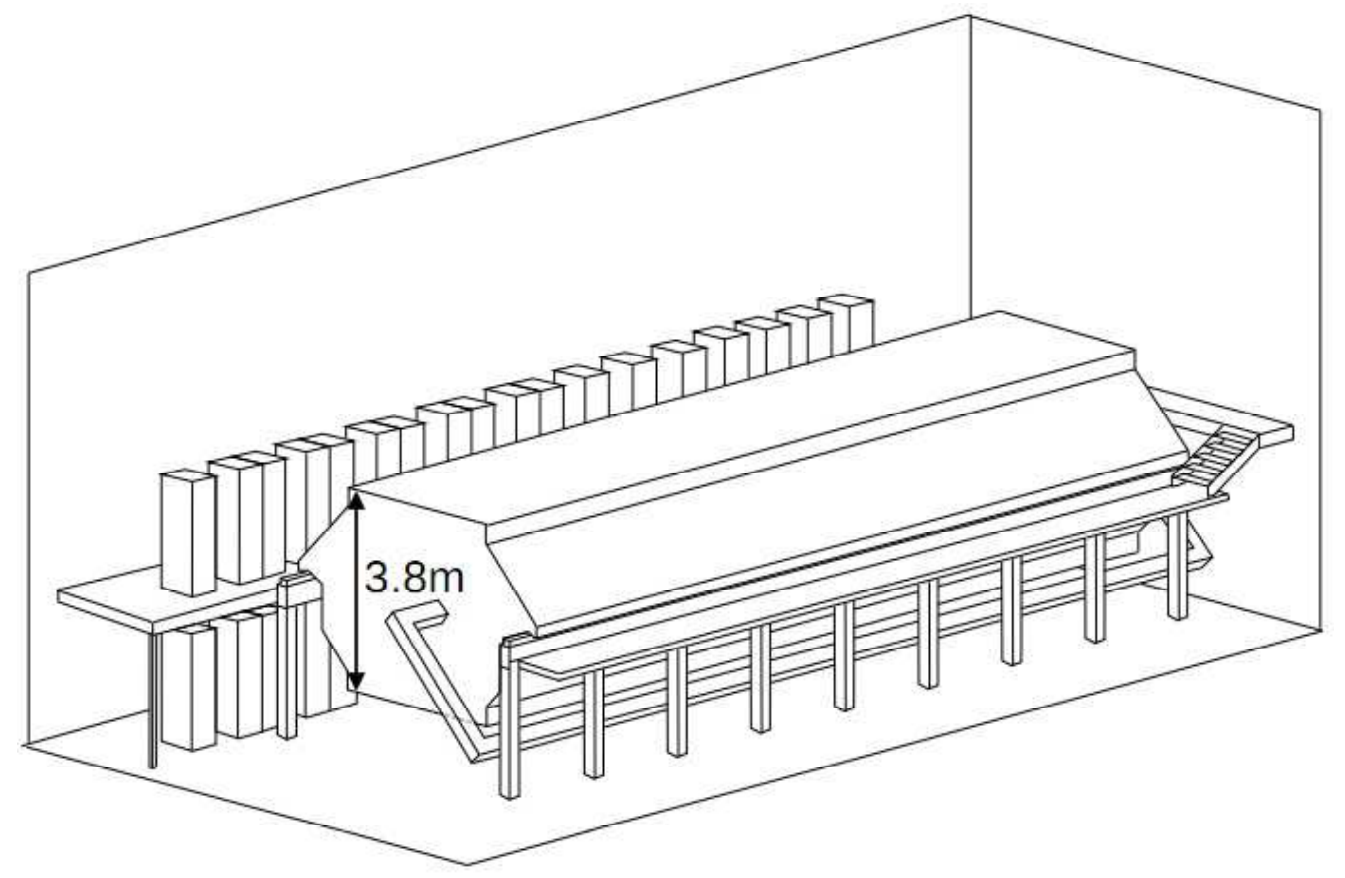

(b) The MINOS Near Detector- 3D view

Figure 3.5: The MINOS Near Detector (ND). Figure taken from [33] and [67] 

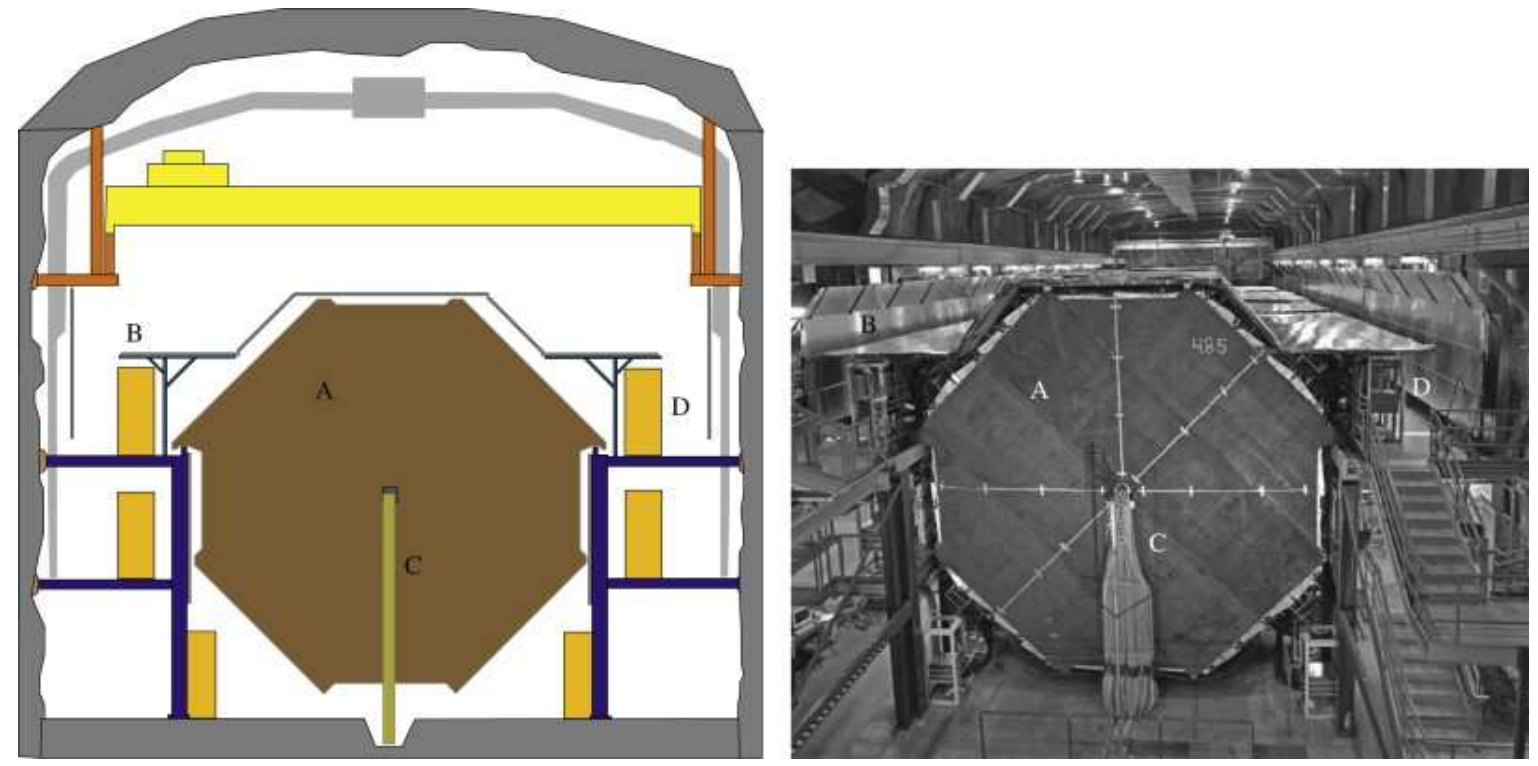

(a) The MINOS Far Detector- cross sectional view diagram (left) and picture (right). Identified are the detector (A), the cosmic ray veto shield (B), the current coil (C) and the electronic racks (D)

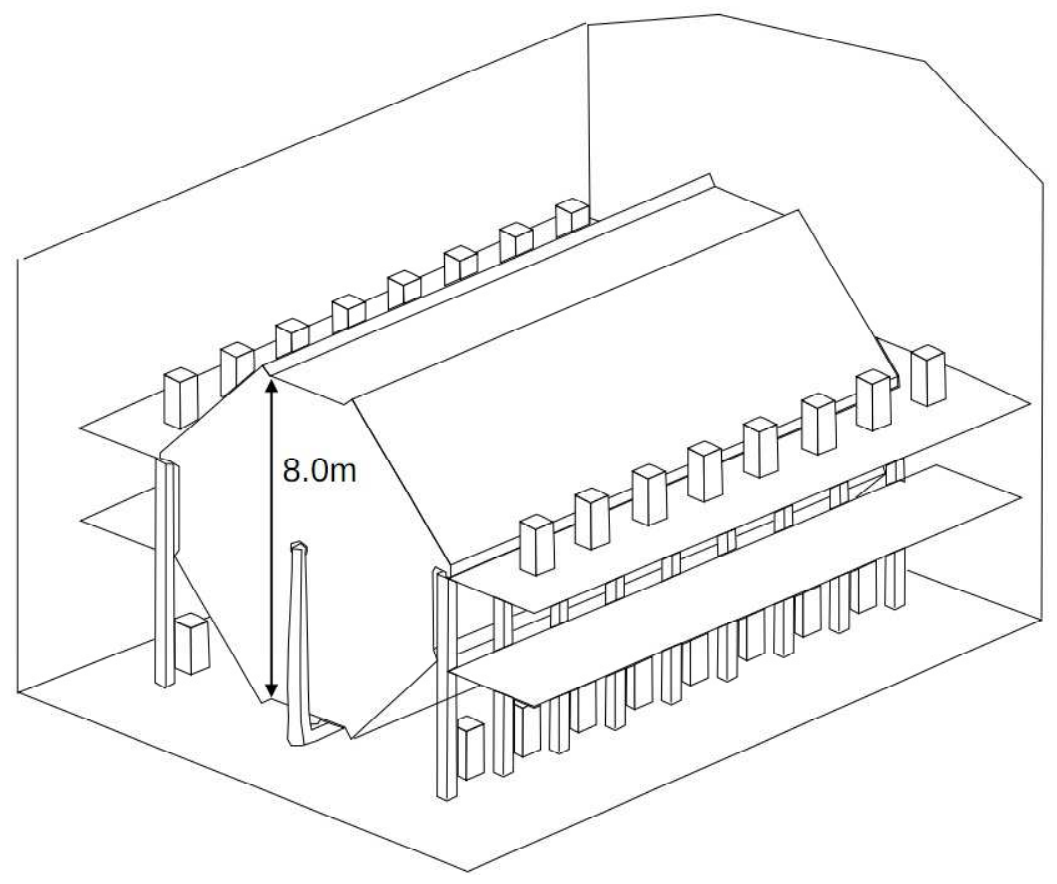

(b) The MINOS Far Detector-3D view

Figure 3.6: The MINOS Far Detector (FD). Figure taken from [33] and [67]. 

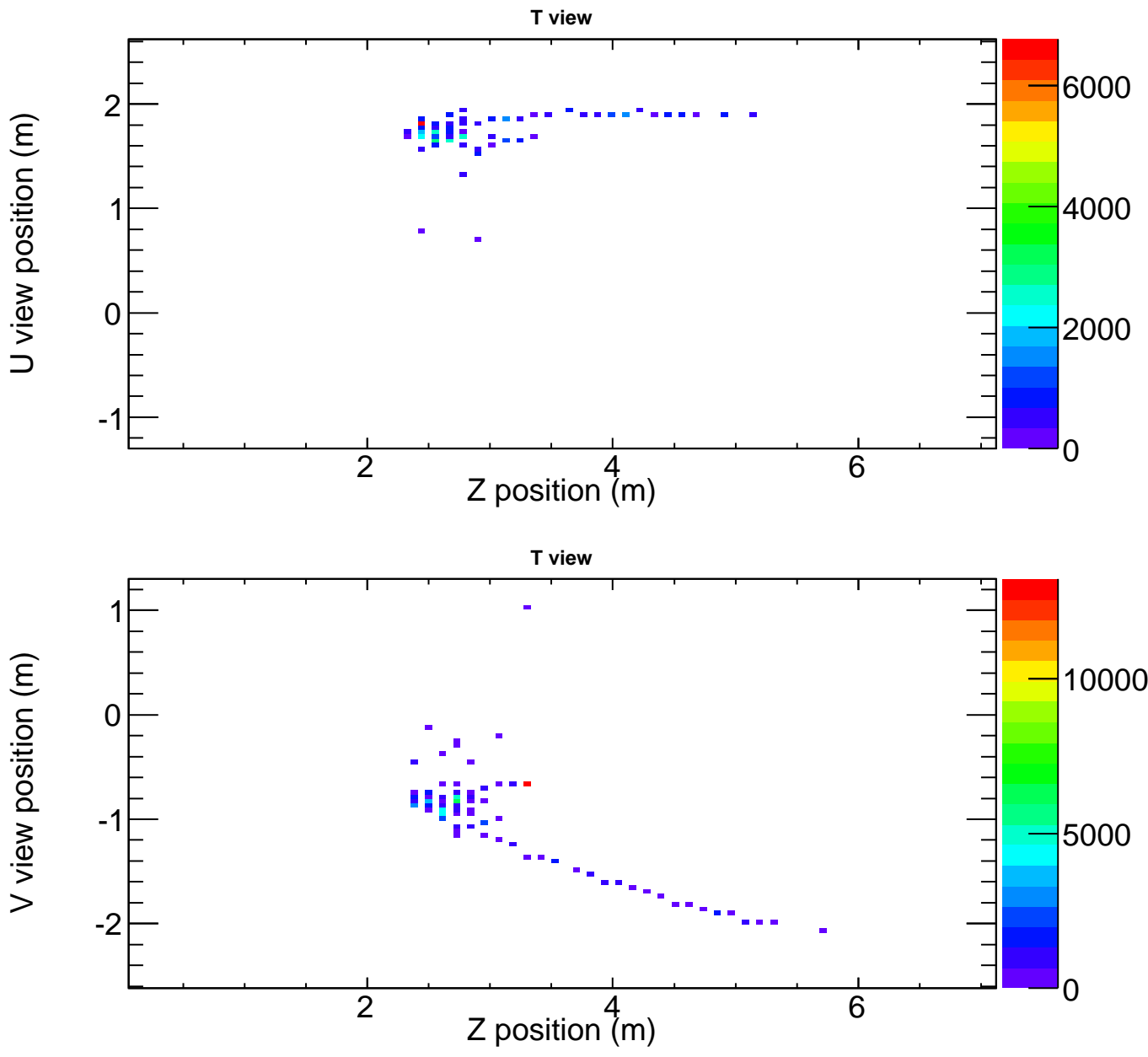

Figure 3.7: A simulated charged current deep inelastic scattering event in the MINOS Near Detector (ND). The true neutrino energy is $10.5 \mathrm{GeV}$ and the reconstructed energy is $10.8 \mathrm{GeV}$. 

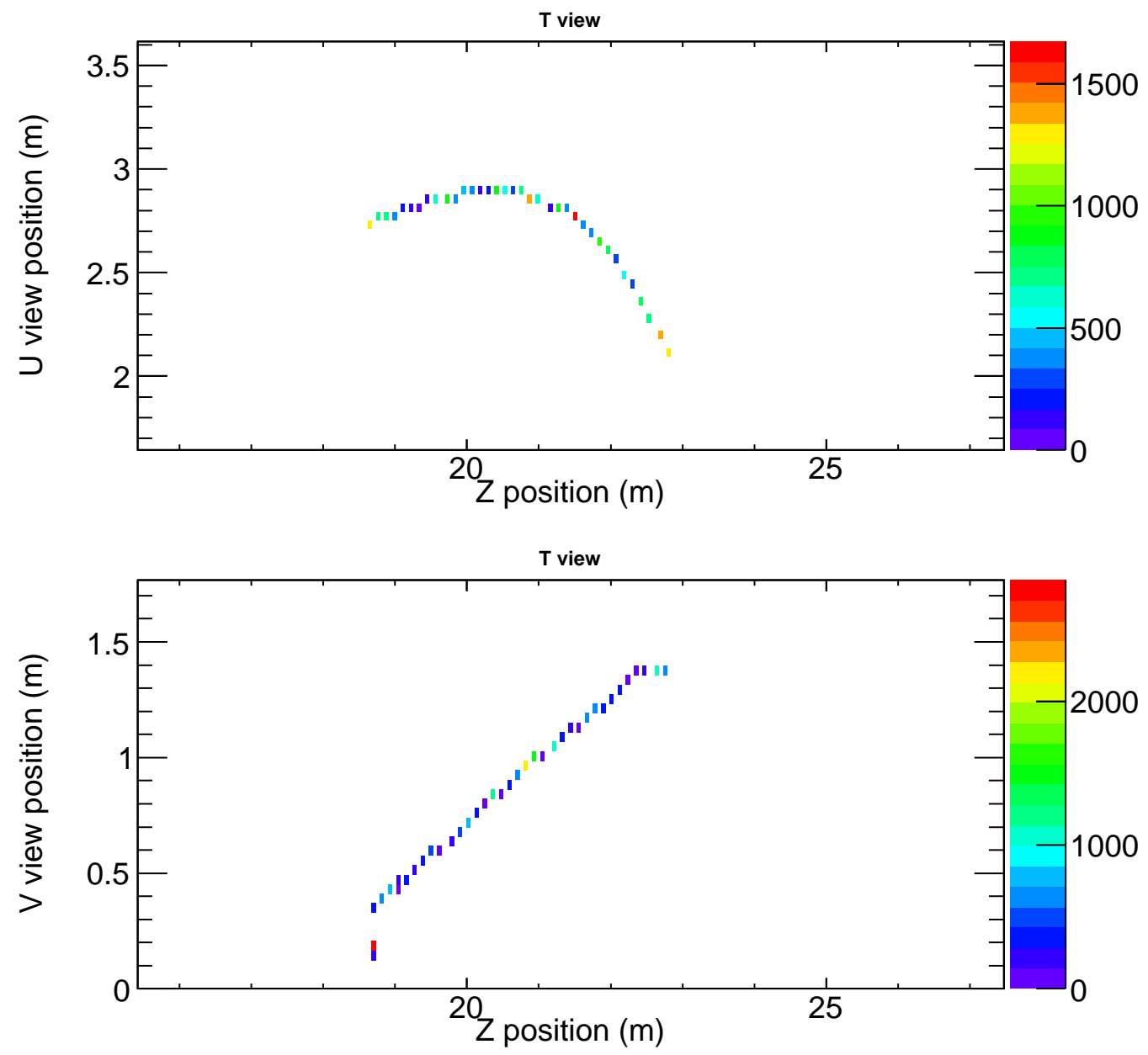

Figure 3.8: A charged current quasi-elastic event in the MINOS Far Detector (FD). The true neutrino energy is $3.31 \mathrm{GeV}$ and the reconstructed energy is $3.44 \mathrm{GeV}$. 

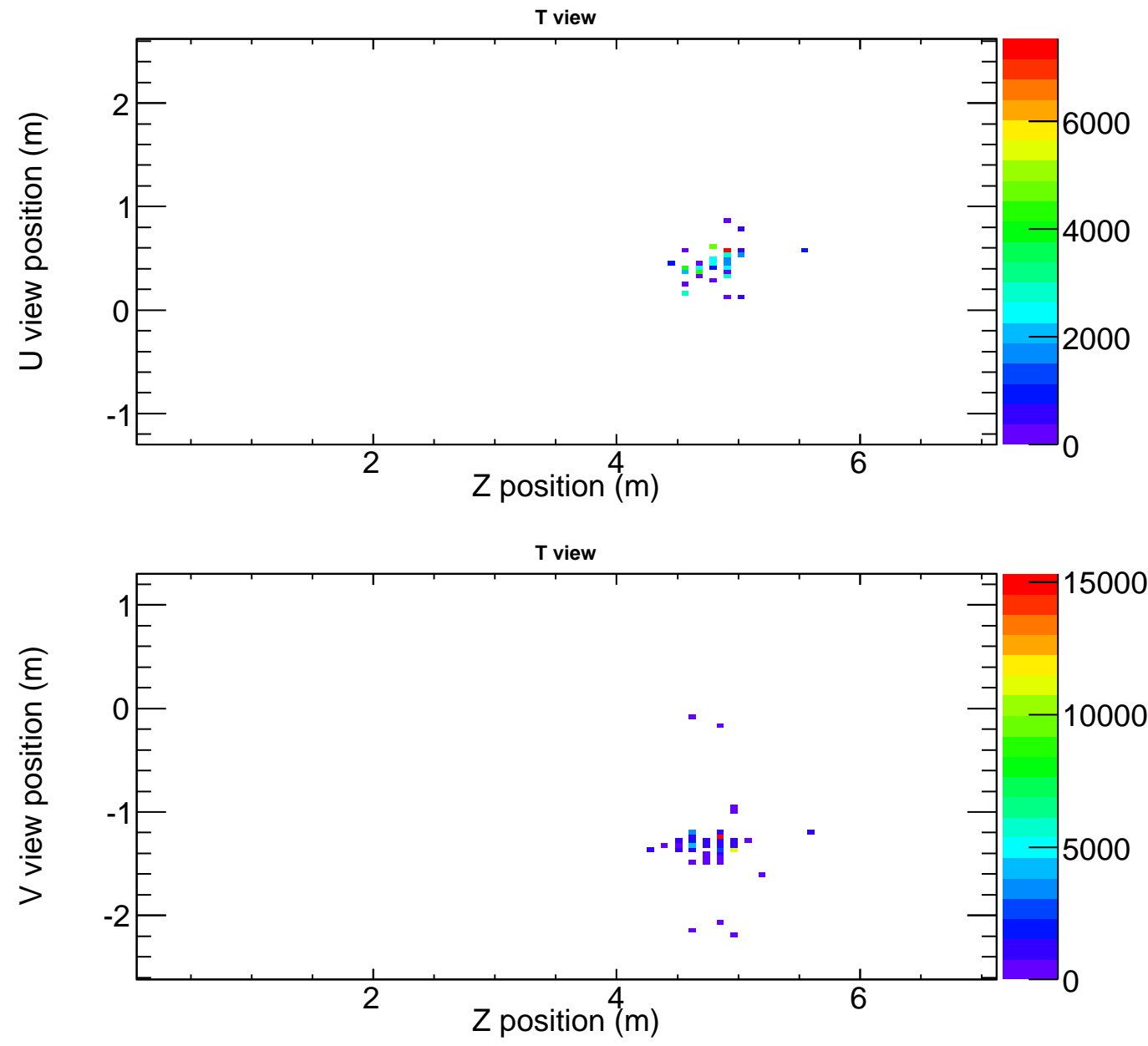

Figure 3.9: A neutral current deep inelastic scattering event in the MINOS Near Detector (ND). The true energy is $13.3 \mathrm{GeV}$ and the reconstructed energy for calorimetry is $9.51 \mathrm{GeV}$. 

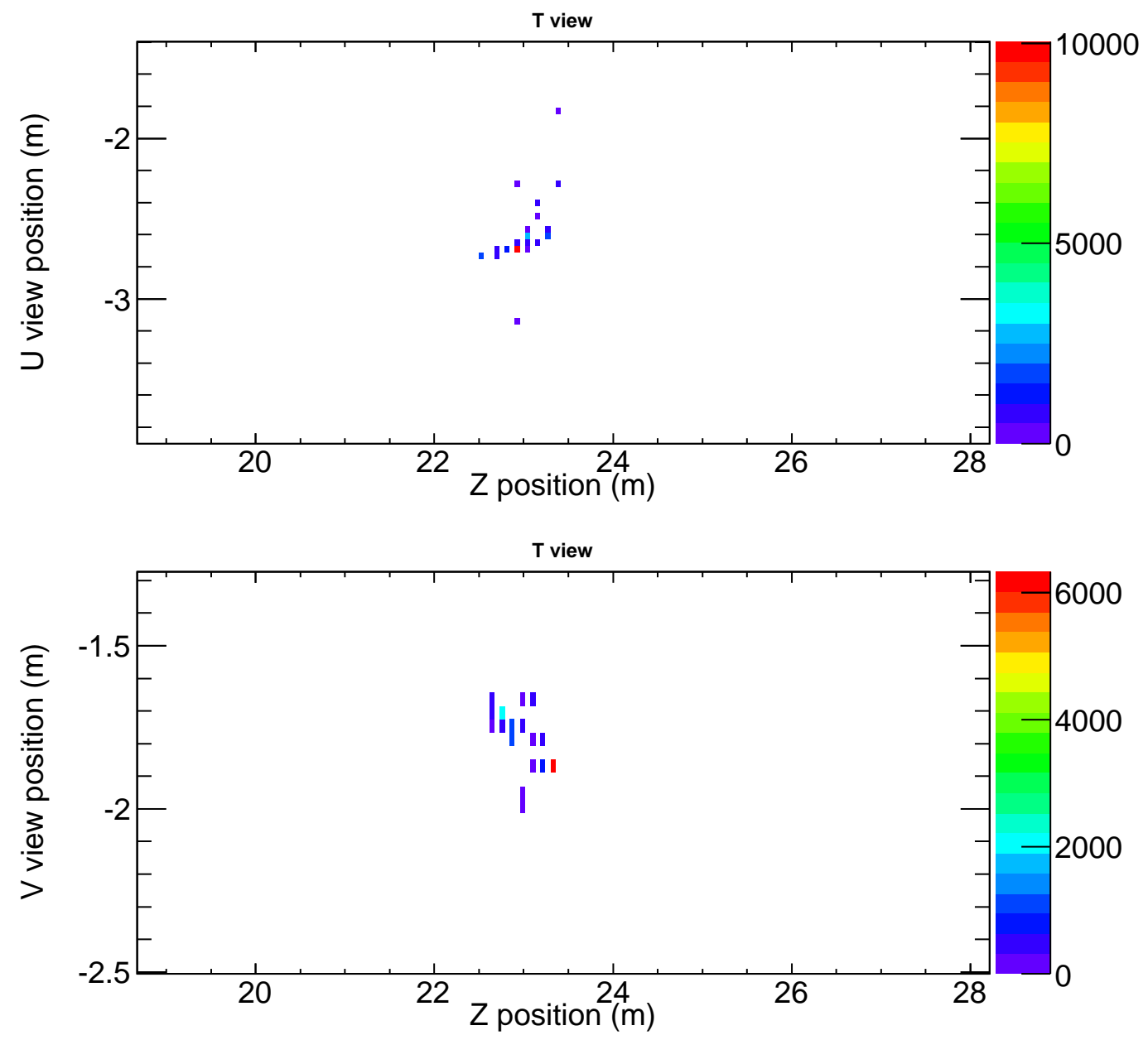

Figure 3.10: A neutral current deep inelastic scattering event in the MINOS Far Detector (FD). The true energy is $22 \mathrm{GeV}$ and the reconstructed energy is $4.5 \mathrm{GeV}$. 
muon. In Figure 3.10 a track is reconstructed in this hadronic shower. This is a potential background to the charged-current $\nu_{\mu}+F e \rightarrow \nu_{\mu}+X$ sample selection.

\subsubsection{The MINOS Fiducial Volumes}

Generally, the MINOS experiment only uses events whose reconstructed vertex is within a fiducial volume. This helps ensure that the events MINOS considers for analysis are well understood and instrumented region. Events outside the fiducial volume of each detector have poor energy resolution and are usually not suited for use in an oscillation analysis. Later, we will briefly describe an analysis which will use events outside of the fiducial volume of the Far Detector.

The fiducial volume for the Near Detector is the cylindrical volume defined by a circle with radius $1 \mathrm{~m}$ centered about the NuMI axis, which is roughly $1.48 \mathrm{~m}$ from the Near Detector coil in the $\hat{x}$ direction and $0.24 \mathrm{~m}$ in the $\hat{y}$ direction. Because the fiducial voume follows the path of the NuMI beam, it slants downward at a rough 3.3 degree angle from horizontal. The front edge of the cylinder is $1 \mathrm{~m}$ downstream of the front face of the Near Detector. The Z-axis of the cylinder is roughly $4 \mathrm{~m}$.

The fiducial volume for the Far Detector is separated into two nonintersecting volumes - one for each supermodule. In the Far Detector, the coil is at the center of the detector. Therefore, a torus, rather than cylinder shape is used to exclude events with vertices too close to the coil. For each

supermodule, the torus has an outer radius of $\sqrt{14} \mathrm{~m}$ and an inner radius of $0.5 \mathrm{~m}$. The first four planes of each supermodule are excluded as well as the last eight planes of the first supermodule and the last twenty planes of the 
second supermodule.

\subsection{The MINER $\nu$ A Detector}

The MINER $\nu \mathrm{A}$ detector is designed for precision measurements of neutrino cross sections. It is located about 2 meters in front of the MINOS Near Detector and is centered along the NuMI z axis. The MINER $\nu$ A detector is composed of several sub-detectors, each with different functionality in mind. A sub-detector is built from multiple cross section modules and the active, data collecting region of the detector can be thought of as a series of module combinations with every module composed of different materials, but the same hexagonal geometry . The stacked modules together make a hexagonal prism shape, as seen in Figure 3.11. The composite stacked-module detector stands roughly $3.5 \mathrm{~m}$ wide by $4 \mathrm{~m}$ tall with a longitudinal (along the beam-line) width of $4.6 \mathrm{~m} .{ }^{5}$ The materials and design of MINER $\nu \mathrm{A}$ is similar to MINOS in that the detector is composed of planes of scintillator strips with alternating directions along with planes of heavier, passive target material.

The MINER $\nu \mathrm{A}$ detector is comprised of an inner detector (ID) and an outer detector (OD). Each MINER $\nu \mathrm{A}$ module contains cross section of the ID and OD sub-detectors (see Figure 3.12). As is perhaps suggested by the name, the OD surrounds the ID and has the geometry of an "empty hexagon". The OD is divided into six towers. Each tower covers a single side of the MINER $\nu \mathrm{A}$ ID and adjacent towers are at a 120 degree angle. The length of the tower increases with radius, such that the gaps between adjacent towers remain constant. Within the OD towers, scintillator strips are placed

\footnotetext{
${ }^{5}$ The MINER $\nu$ A experiment has not published a detector-description paper yet. Most of the information here comes from their unpublished technical design report [68].
} 


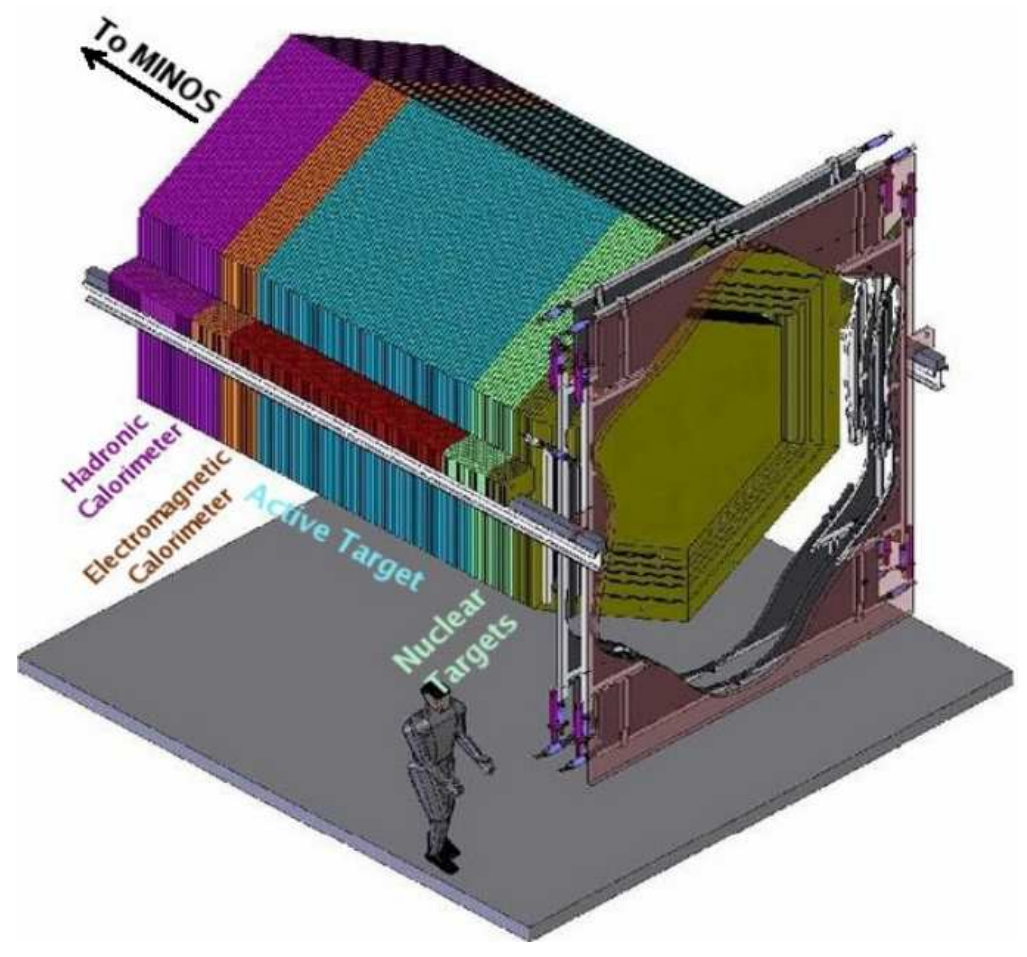

Figure 3.11: A three dimensional representation of MINER $\nu$ A. This figure is taken from the conceptual design report [68]. After this conceptual design was made, modifications to the MINER $\nu$ A detector design took place. This included the addition of a upstream passive helium target, and a veto shield 
perpendicular to the radius at the middle of the tower (which is parallel to the sides of the hexagon). The distance between strips ends in neighboring towers is roughly $2.79 \mathrm{~cm}$. The scintillator strips are grouped in doublets. Each strip is $1.9 \mathrm{~cm} \times 1.5 \mathrm{~cm}$ in width. Each doublet is called a "story" and there exist 4 stories in every detector. Because the OD increases in length with radius, the scintillator strips in each story are of different lengths. The first story, which is closest to the inner detector, has scintillator strips which are 52.66 $\mathrm{cm}$ long, the second story has scintllator strips which are $56.38 \mathrm{~cm}$ long, the third story has scintillator strips which are $60.16 \mathrm{~cm}$ long, and the fourth story has scintillator strips which are $66.50 \mathrm{~cm}$ long. ${ }^{6}$

The ID is similarly hexagonal in shape with an apothem of $41.9 \mathrm{~cm}$. A gap of $1.475 \mathrm{~cm}$ separates the inner detector from the outer detector. Each instrumented module contains two planes of 127 scintillator strips. Unlike the MINOS detectors which have two views, the MINER $\nu \mathrm{A}$ detector employs three independent views, $\mathrm{X}, \mathrm{U}$ and $\mathrm{V}$. The $\mathrm{X}$ view is equivalent to the NuMI defined $\mathrm{X}$ view coordinate. The $\mathrm{U}$ view is defined by $u \equiv(x-\sqrt{3} y) / 2$ and the $\mathrm{V}$ view is defined by $v \equiv(x+\sqrt{3} y) / 2$. Each module contains two of these views, either $\mathrm{X}$ and $\mathrm{U}$ or $\mathrm{X}$ and $\mathrm{V}$. The overlap of information between these three views allows MINER $\nu$ A to verify spatial information of energy deposits. The MINER $\nu \mathrm{A}$ strip coordinate system $(\mathrm{X}, \mathrm{U}, \mathrm{V})$ can be compared to the MINOS coordinate system. These are shown side by side in Figure 3.13.

By changing the material in the module ID area, the MINER $\nu \mathrm{A}$ experiment is able to further subdivide the ID into sub-detectors. (see Figure 3.14 These are:

\footnotetext{
${ }^{6}$ These numbers come from technical drawings by Robert Flight, at the University of Rochester.
} 


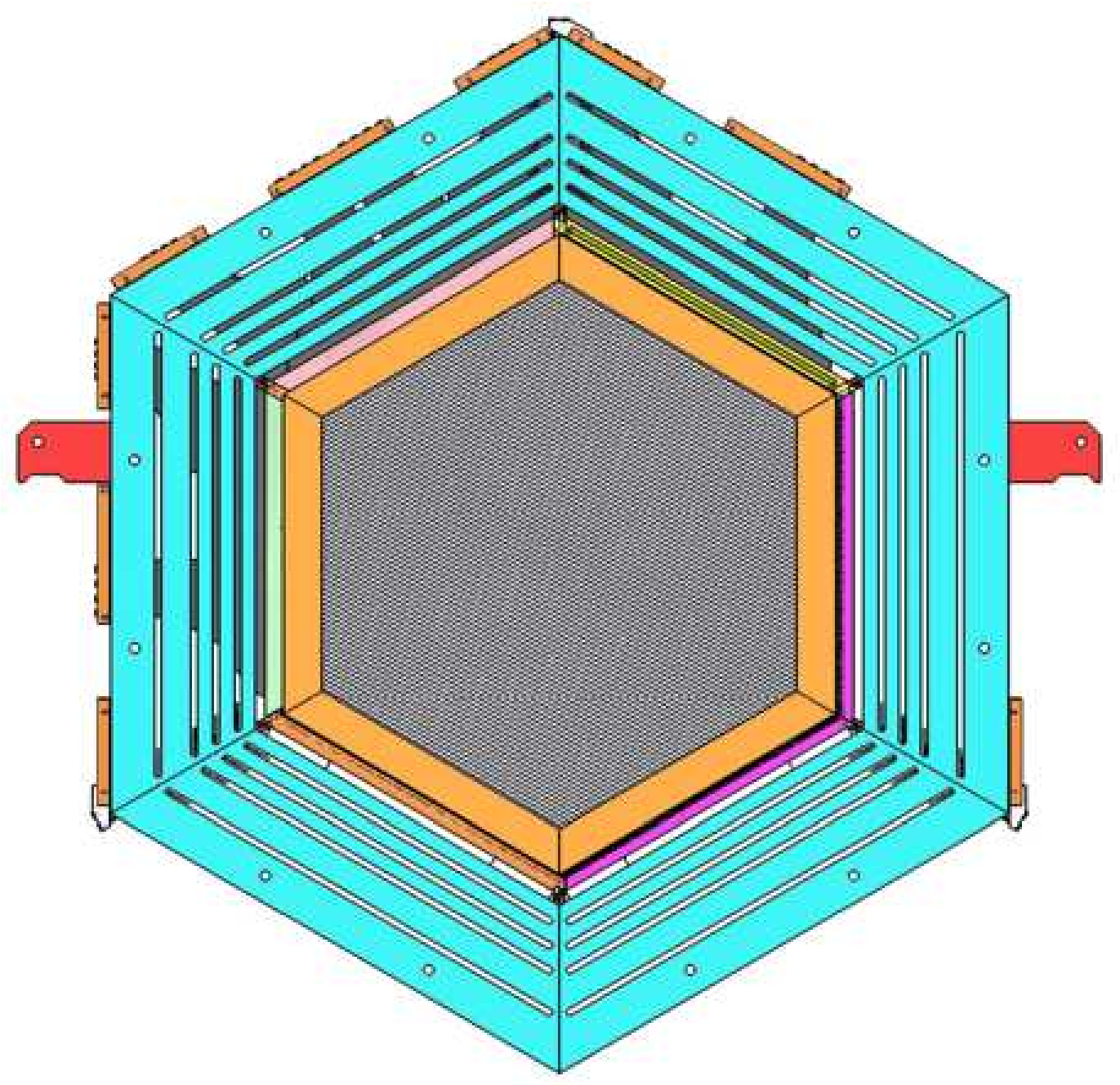

Figure 3.12: A single minerva module - beam view. The grey region is the 'inner detector' region, which can be composed of scintillator strips or various passive target materials. The blue region which surrounds the inner detector region is the outer detector. The outer detector is composed of six towers, one for each side of the hexagon. This steel frame has four 'stories' of scintillator strips, which are parallel to the sides of the inner detector. Hooks on the side are used to hang each module(shown in pink). 


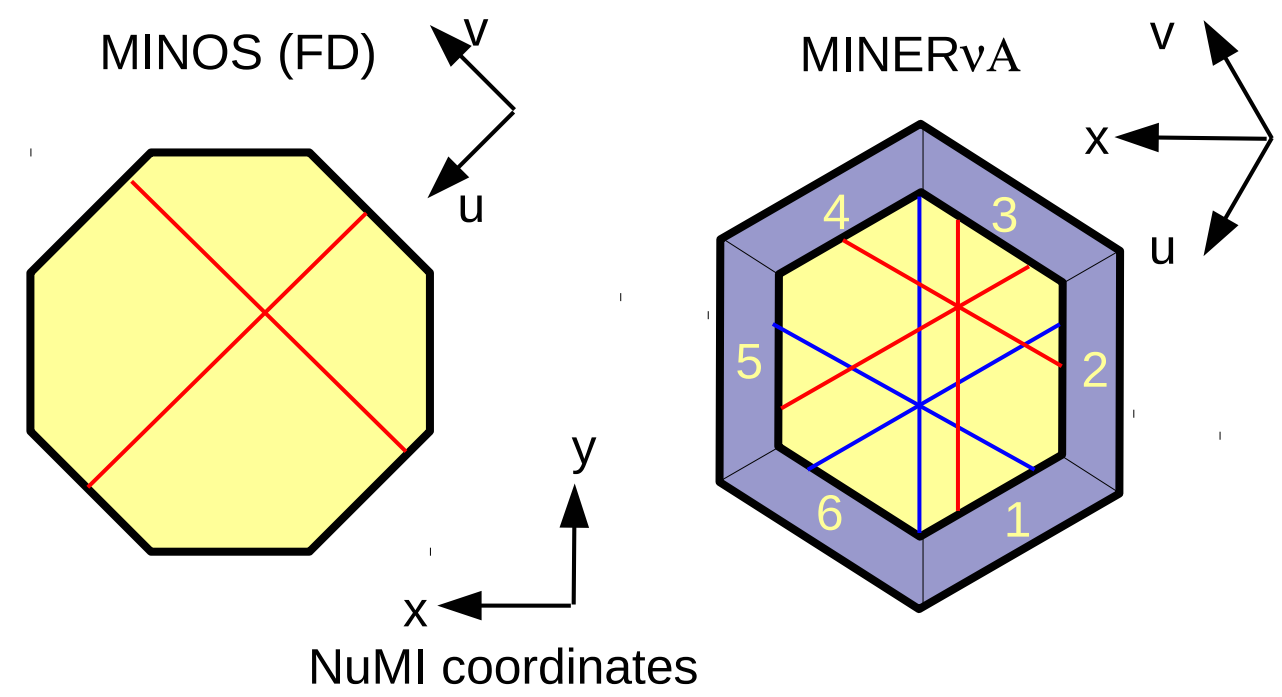

Figure 3.13: A diagram of the MINOS and MINER $\nu$ A detectors. Here the local coordinate systems are explicitly drawn next to the cross section of the detector. For the MINER $\nu$ A detector, the outer detector stories are labeled (1$6)$. The lines drawn represent scintillator strips. Scintillator strips in different views together specify a position in the detector. For MINOS the two views are orthogonal. For MINER $\nu \mathrm{A}$, the three views are rotated 60 degrees from each other. The blue and red lines demonstrate that this extra view helps sort out combinatorial problems in multi-track events 
- A 'scintillator only' Tracker Region, to obtain high resolution. Each module is composed entirely of planes of scintillator strips.

- A scintillator and passive Nuclear Target material region upstream of the Tracker region. Each module is composed of a combination of nuclear target materials or scintillator strip planes. Passive nuclear target modules are separated by multiple scintillator modules. The passive targets include, iron, lead, water, carbon, and helium. Each target module has a unique combination of these materials.

- An electromagnetic calorimeter (ECAL) region directly downstream of the Tracker region. Each module is composed of scintillator planes with a $0.2 \mathrm{~cm}$ lead sheet applied per scintillator.

- A hadronic calorimeter (HCAL) region directly downstream of the ECAL region. Each module is fitted with scintillator strip planes and a 1-inch thick iron sheet is applied per plane.

Additionally, Tracker region modules, a second 'frame' is placed $30 \mathrm{~cm}$ from the edge of the ID and $0.2 \mathrm{~cm}$ thick at the beginning of each module and between scintillator planes. This region is called the Side ECAL. A veto plane at the beginning of MINER $\nu \mathrm{A}$ detects incoming particles to help identify muons that did not originate from neutrino events in the MINER $\nu \mathrm{A}$ detector. After the initial design of the MINER $\nu \mathrm{A}$ detector, a helium tank was added as an additional upstream target between the first veto wall and the first module. The helium is uninstrumented and acts as a passive target. These changes can be seen in Figure 3.15.

In the MINER $\nu \mathrm{A}$ ID, instead of the rectangular scintillator strips used by the MINOS experiment, the MINER $\nu \mathrm{A}$ scintillator strips are triangular 


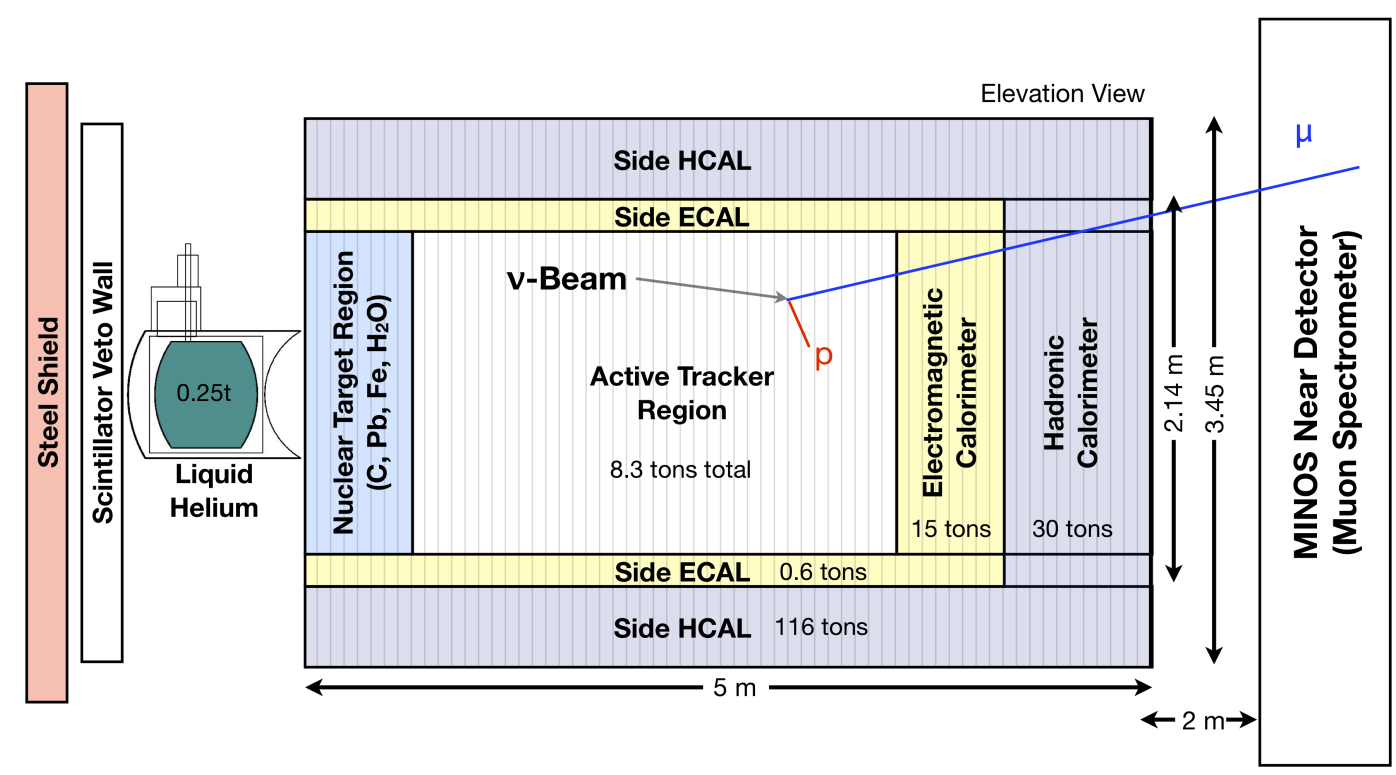

Figure 3.14: A schematic of the MINER $\nu \mathrm{A}$ detector with the different regions of the detector labeled.

with a maximum length of $3.3 \mathrm{~cm}$ and a minimum base to corner length of $1.4 \mathrm{~cm}$. These scintillator strips are fitted such that a plane with height 1.4 $\mathrm{cm}$ is created. A wavelength shifting fiber in the center of the scintillator strip carries light from the scintillator strip to a PMT, as in the MINOS experiment. The purpose of the redesigned strips was to obtain higher resolution spatial information using the energy left in each scintillator as a measure of the muon direction.

The advantage of the MINER $\nu \mathrm{A}$ geometry requires more explanation. The downstream and side ECAL and HCAL regions were proposed to increase containment of high-energy hadronic particles and muon tracks. This is of particular concern or MINER $\nu \mathrm{A}$ because of the lack of a magnetic field. Downstream of the fully active tracker region, the ECAL region with $2 \mathrm{~mm}$ lead foil absorbers dispersed with scintillator. Additionally, along the sides 


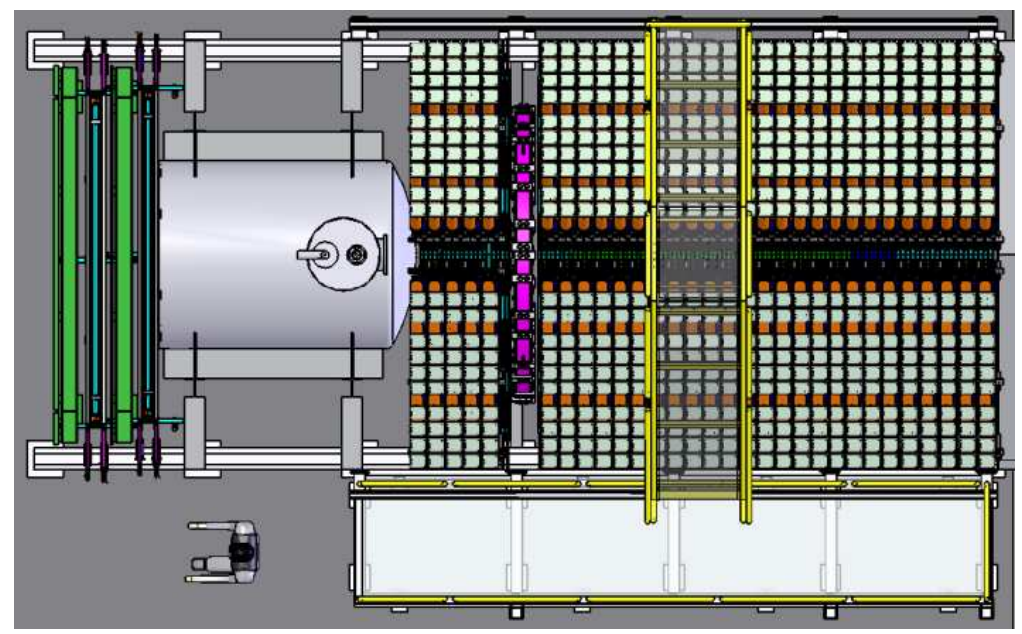

(a) MINER $\nu \mathrm{A}$ as constructed, Top view

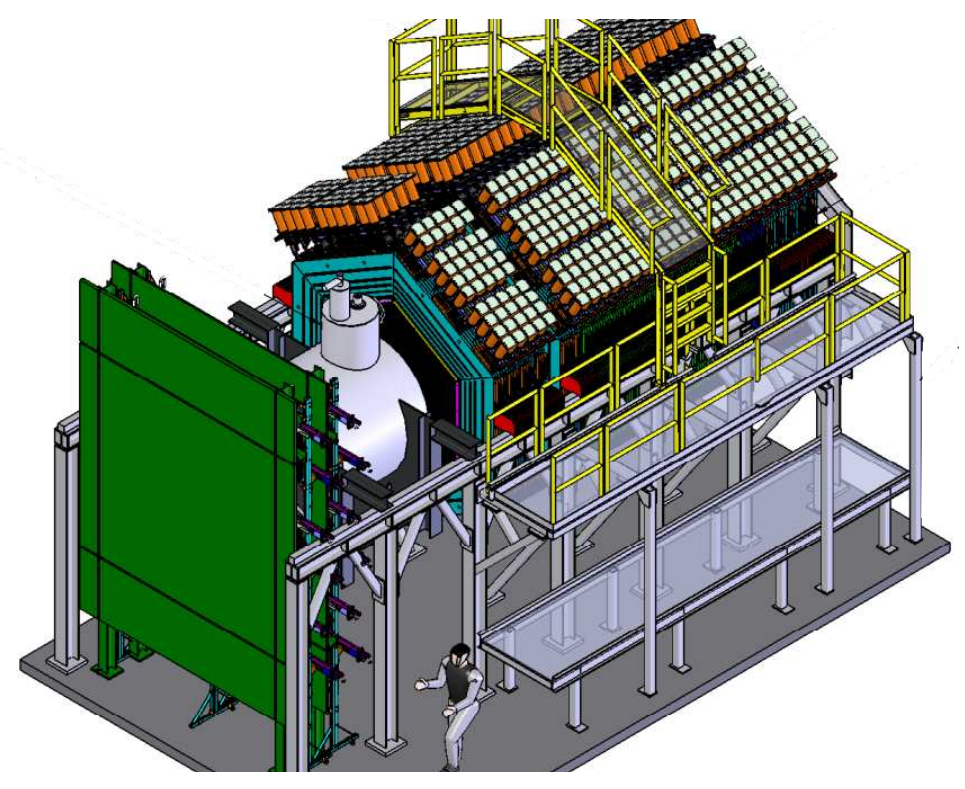

(b) MINER $\nu \mathrm{A}$ as constructed, 3D view

Figure 3.15: The MINER $\nu$ A detector as constructed. These were taken from technical drawings by Robert Flight. (a) shows the MINER $\nu$ A detector from the top. From left to right, the veto shield (in green), the helium target (the grey block), and the modular part of MINER $\nu \mathrm{A}$. (A small water target is seen in purple). (b) shows a 3D view of MINER $\nu \mathrm{A}$. Here auxillary facilities, like the walkway and stairs are shown. 
of thee tracker region module, additional lead foils are place. Downstream of the ECAL resides the HCAL, which has steel modules inter-dispersed with scintillator modules.

As discussed before, modules are composed of an ID region which contains either target or scintillator material and an OD region, which is composed of a steel frame, inter-dispersed with scintillator. The OD scintillator strips are parallel to the sides of the MINER $\nu \mathrm{A}$ hexagon. Each hexagon side is associated with an OD "Tower", as shown in Figure 3.11. The radial geometry of the OD allows the MINER $\nu \mathrm{A}$ experiment to monitor particle trajectories as they leave the detector in the direction they are lost. The directions of the outer detector scintillator strips reflects the possible directions of the inner detector strips - 3 distinct directions exist.

The MINER $\nu \mathrm{A}$ experiment uses the MINOS Near Detector as an additional calorimeter for high-energy muons that have a high probability to leave through the downstream end of the MINER $\nu \mathrm{A}$ detector. Because MINOS has a magnetic field, muons that exit MINER $\nu \mathrm{A}$ and enter MINOS through the front face are likely to be well contained. Even those that are not well contained have good energy estimates through measuring the curvature of th muon. In Figure 3.16 a neutrino event that begins in the MINER $\nu \mathrm{A}$ detector produces a muon that travels out the back of the MINER $\nu \mathrm{A}$ detector and enters the MINOS detector.

The visualization of neutrino events in the MINER $\nu \mathrm{A}$ detector is much finer than in the MINOS detector. Figure 3.18 shows a neutral current event (with high energy) where the hadronic particles are clearly seen in the detector. Similarly Figure 3.19 shows a charged current event with multiple particle tracks, only one of which is caused by a muon. This demonstrates the need for 
a refined particle recognition algorithm which separates charged current and neutral current events and futher identifies the muon with complex charged current scatters. 

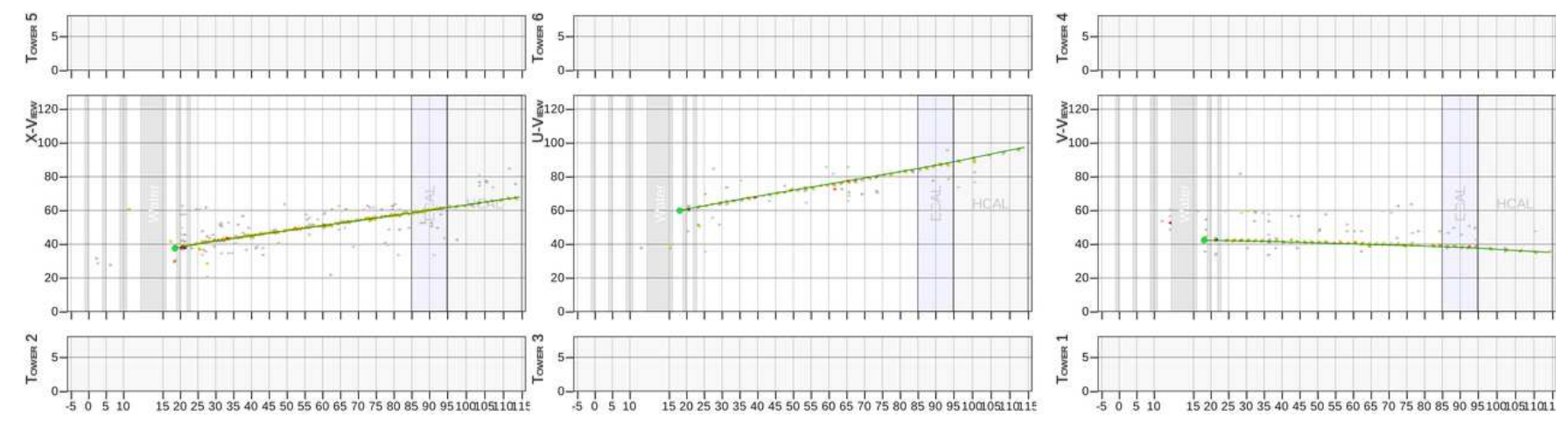

Minos U View

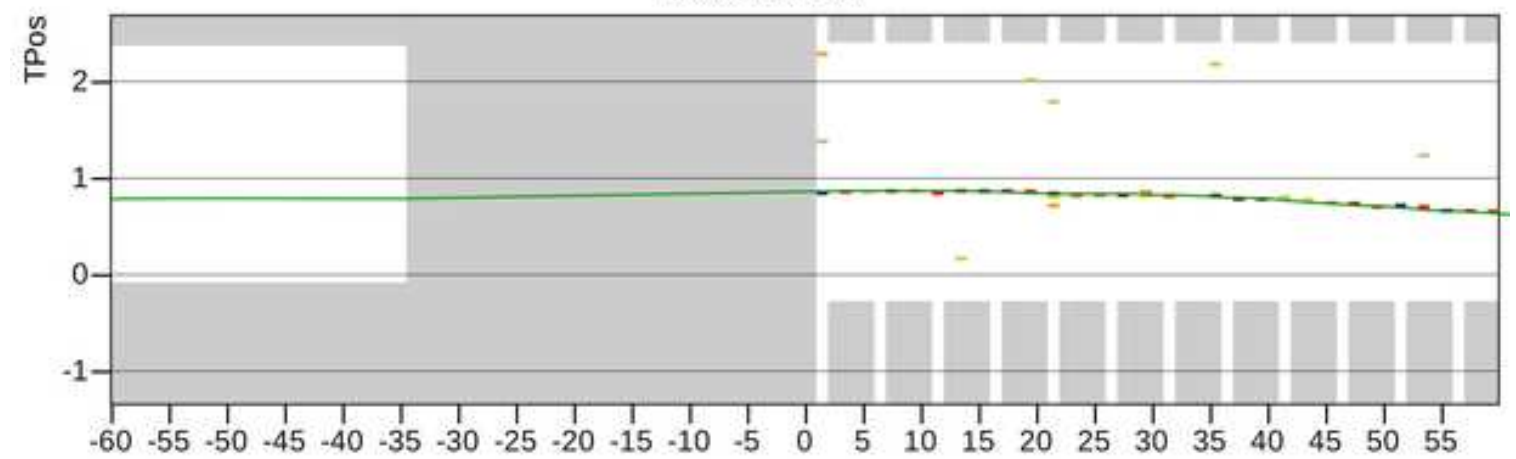

Figure 3.16: Simulation of a charged current quasi-elastic neutrino event in the MINER $\nu \mathrm{A}$ detector. This event has an ideal topology, where the muon enters the MINOS Near Detector where its energy and sign can be easily determined 

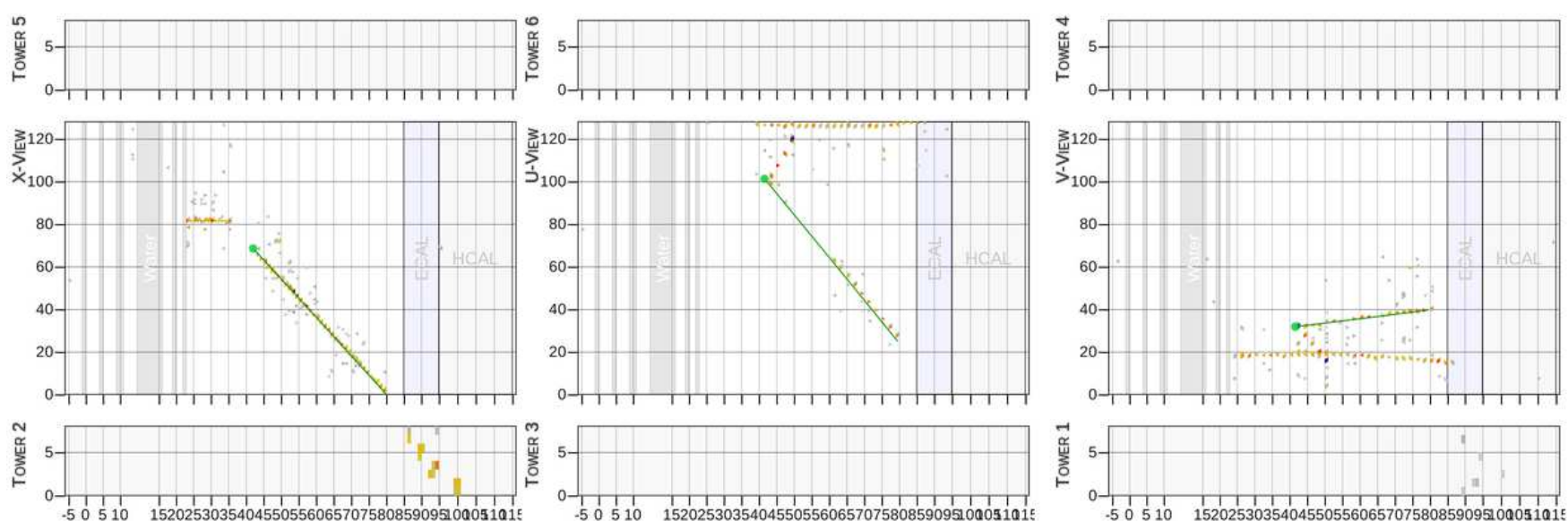

Figure 3.17: A neutrino event in the MINER $\nu$ A detectors where a long, minimum ionizing track indicative of a muon is seen leaving the detector. The event begins in the Tracker region and leaves through the side ECAL. It then traverses the OD region before leaving the detector. $\mathrm{X}, \mathrm{U}$, and $\mathrm{V}$ views are shown from right to left. Note that another unreconstructed event is seen. 

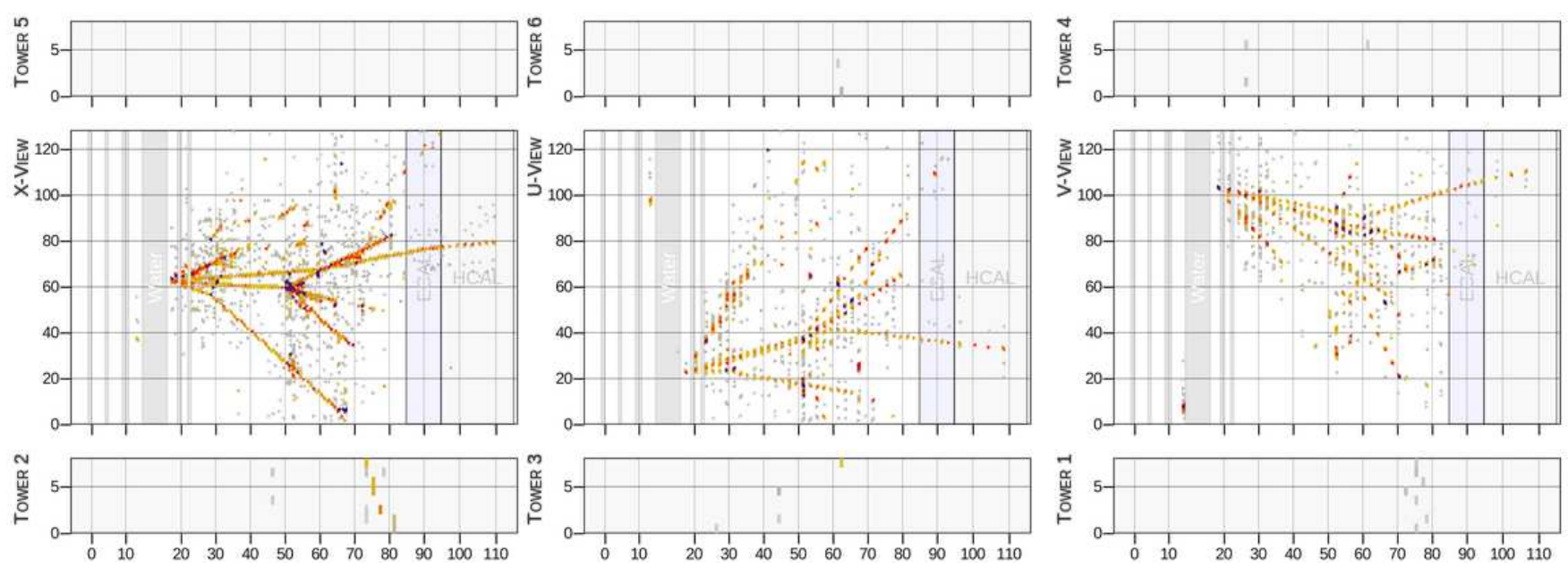

Figure 3.18: A simulated $21 \mathrm{GeV}$ NCDIS neutrino interacts in the MINER $\nu \mathrm{A}$ detector. Visible final state particles include a $4.3 \mathrm{GeV} \pi^{+}$, a $1.3 \mathrm{GeV} \pi^{0}$ that leaves a soft electromagnetic shower and a lower energy pion and proton. A $10 \mathrm{GeV}$ neutrino leaves undetected. The high resolution detector easily visualizes the energetic final state hadrons. A second $\pi+C$ reaction is seen. Reconstructing complex final state interactions is one of the difficulties of MINER $\nu \mathrm{A}$ 

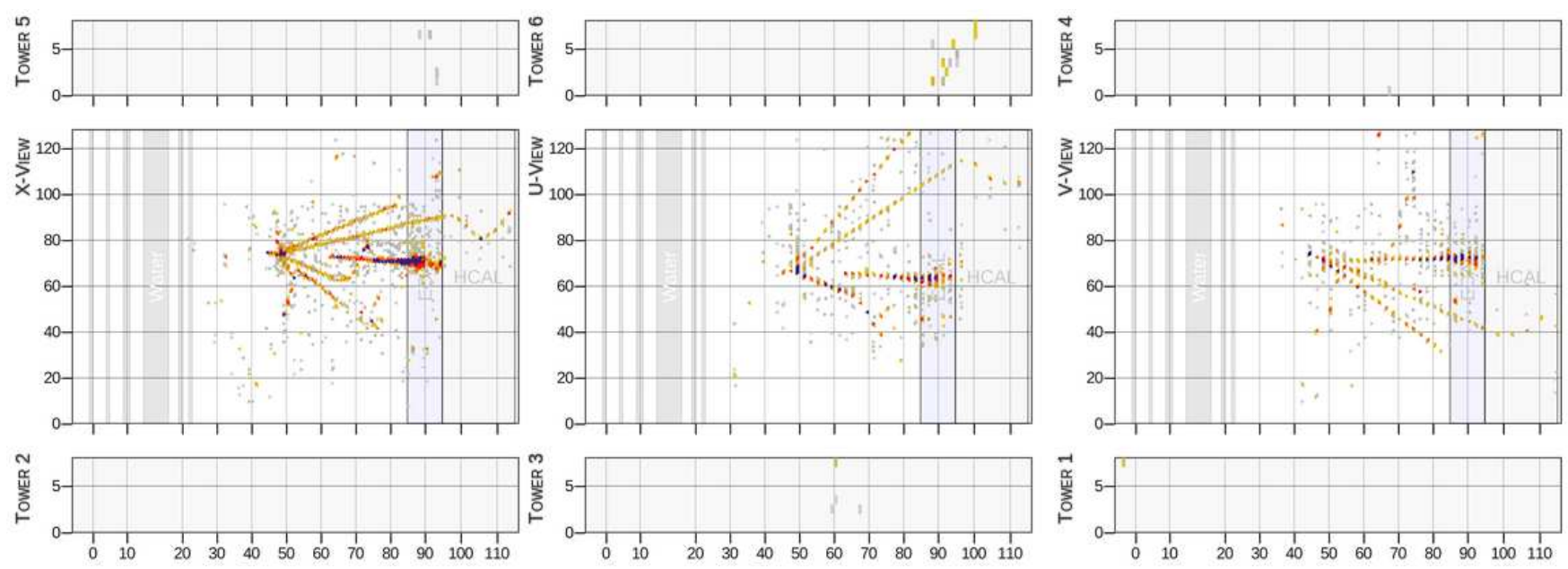

Figure 3.19: A simulated $10 \mathrm{GeV}$ CCDIS neutrino interacts in the MINER $\nu$ A detector. Visible final state particles include an electromagnetic shower from a $3 \mathrm{GeV} \pi^{0}$ decay, a $1.8 \mathrm{GeV}$ pion that multiple scatters in the $\mathrm{HCAL}$, a $1.6 \mathrm{GeV}$ muon that leaves the MINER $\nu \mathrm{A}$ detector through the OD and other lower energy pions 


\section{Chapter 4}

\section{MINOS Charged Current Event Identification}

In order to observe the disappearance of muon neutrinos, it is imperative to separate charged current and neutral current events. NuMI is a source of muon neutrinos. Therefore, we focus on identifying the muons produced in charged current muon neutrino events. It is unlikely that a neutral current event would produce an identifiable muon; most neutral current events appear as hadronic showers in the MINOS detector and have no reconstructed tracks or very short tracks from high-energy hadronic particles. We can reduce

the process of identifying charged current muon neutrino events to identifying muons in events. Events with trackable muons are overwhelmingly charged current muon neutrino interactions. In this chapter, we will discuss methods to identify charged current events through their signature muon track while maintaining a low neutral current contamination fraction. Specifically, variables which separate minimum-ionizing particle tracks from tracks created by hadronic particles are developed and used as inputs to a k-Nearest-Neighbor classification algorithm. 


\subsection{Previous Algorithms for Muon Identification}

The charged current identification method previously used by MINOS was built by Rustem Ospanov [69]. ${ }^{1}$ The method utilizes many of the properties of minimum-ionizing particles discussed in Chapter 3. Muons lose energy almost exclusively through ionization and a muon's ionization rate is flat for energies typical in few $\mathrm{GeV}$ neutrino interactions. Variables which strongly separate muons from other particles typically probe this minimum ionizing behavior.

The work by Ospanov revealed that for the majority of neutrino energies relevant to MINOS, three traits which were strongly correlated with minimum ionizating muons in independent ways. That is, the trait is not strongly correlated with the presence of another trait by mathematical identity. Therefore, the use of multiple identifying traits provides independent verification of a particular track's muon-likeness. The first trait was the length of the ionization track; with a typical energy loss of $16 \mathrm{MeV}$ per plane in the MINOS detector and the absence of nuclear interactions, a $1 \mathrm{GeV}$ muon will traverse up to 60 planes of the detector. This is a muon's most obvious feature and the easiest one to quantify; we use the number of active (scintillator) planes the track has passed through as the first measure of minimum ionization. In Figure 4.1(a), we contrast a Monte Carlo depiction of track lengths for a charged current muon-like track with tracks identified as hadron particles using the Monte Carlo truth information.

The second trait which probed minimum ionization muons was the average amount of energy lost along their track. Because muons are mini-

\footnotetext{
${ }^{1}$ The analysis was a critical component to the $2008 \operatorname{MINOS} \nu_{\mu}$ disappearance analysis.
} 
mum ionizing, the energy lost per plane in MINOS is nearly the same for all muons. Earlier this was calculated to be around $15 \mathrm{MeV}$ per centimeter of steel (and about 1/7th of that in plastic. see Figure 3.3). This value is well known and near constant with muon energy. The variable which probes this facet of minimum ionization for muons is the mean energy per plane. The energy is summed in units of "minimum ionizing particle energy" or MIPs and, therefore, the final variable is in units of MIPs/plane. In Figure 4.1(b), we contrast the average energy behaviour of muon and hadronic particle tracks. This demonstrates the separation of this variable.

The third trait which was correlated with a muon's minimum ionizing trail was the fluctuation in energy loss along the length of the track. Energy loss through ionization has a well-known shape - the Landau distribution. The Landau distribution allows for fluctuations in energy along the length of a track for a minimum-ionizing particle. However, hadronic particles undergo nuclear interactions. Nuclear interactions, such as $\pi^{-}+p \rightarrow \pi^{0}+n$ or $K^{-}+p \rightarrow K^{0}+n$, result in large energy depositions. Furthermore, the width of the Landau distribution is proportional to $1 / \beta$. Therefore, lower $\beta$ particles, like pions and protons, will have larger fluctuations. Because the Landau distribution is asymmetric and these particles already have a higher mean energy loss through the Bethe-Bloch formula, it is more likely that pions and protons will exhibit large spikes in energy along their track. In order to quantify this, MINOS takes the energy deposited on each plane by the muon track and orders the ionization energy from least to greatest. The vector is divide in half (from 0 to $n / 2$ and $n / 2$ to $n$, where $n$ is the number of ionization planes) and the two halves are summed and divided such that the signal fluctuation variable is defined as $V_{l h}=\sum_{i<n / 5} x_{i} / \sum_{i \geq n / 5} x_{i}$. This variable is shown in Figure 4.1 
on the bottom left.

In addition to these three ionization-based discriminators, a fourth variable was created to ensure that a particle was not closely associated with a shower. This reflects the topological differences between charged current and neutral current events. charged current events consist of a hadronic shower and a separate muon track whereas neutral current events consist soley of a hadronic shower. Therefore, any track from a neutral current event should be closely associated with the shower. It is possible for a hadronic particle from that shower to be energetic enough to lose energy through minimum ionization. The muon should be less associated with the hadronic shower than a particle created from within. This variable was called the "track/plane pulse height" variable, defined as the ratio of the sum of the energy on the track to the sum of the energy on and near the track (within 4 scintillator strips). For example, in the event shown in Figure 3.7, a track is shown which would have a low value for this ratio if one considered only hits below $\mathrm{Z}=2.5 \mathrm{~m}$ because there is an abundance of nearby shower energy. After $Z_{i} 4.0$, the ratio for this same event would increase towards 1.0 , since no nearby shower energy is seen. This variable is shown in Figure 4.1 on the bottom right. ${ }^{2}$

These four discriminating variables were combined using a k-NearestNeighbor $(\mathrm{k}-\mathrm{NN})$ technique. The k-Nearest-Neighbor technique is described in Appendix A. This technique allows one to create a single separation parameter from the individual 'input' discriminating variables. A set of Monte Carlo training events and their given values for the four discriminators is given to the $\mathrm{k}-\mathrm{NN}$ algorithm. The separation parameter value for each event is the

\footnotetext{
${ }^{2}$ Pulse height refers to a measure of the activity in a scintillator strip and is for our purposes equivalent to the energy deposited in arbitrary units.
} 


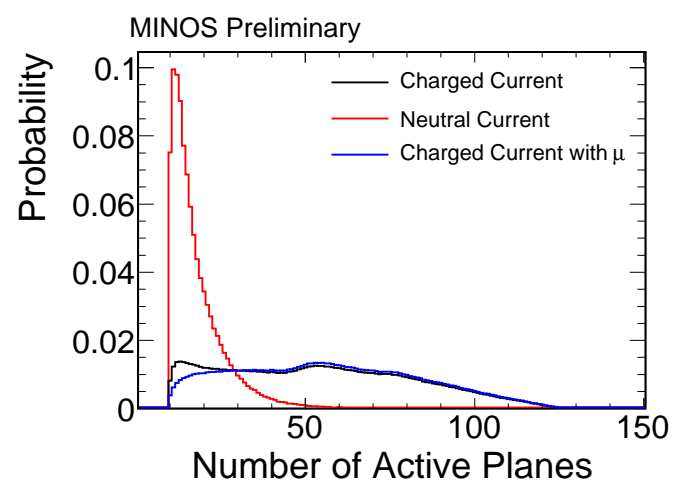

(a) Number of Active Planes

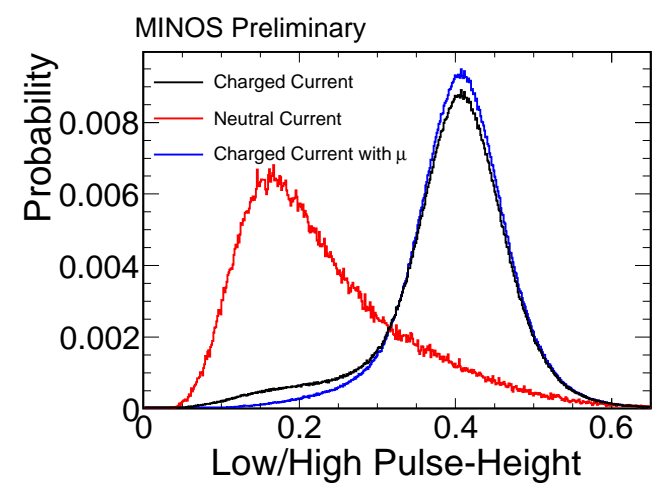

(c) Signal Fluctuation

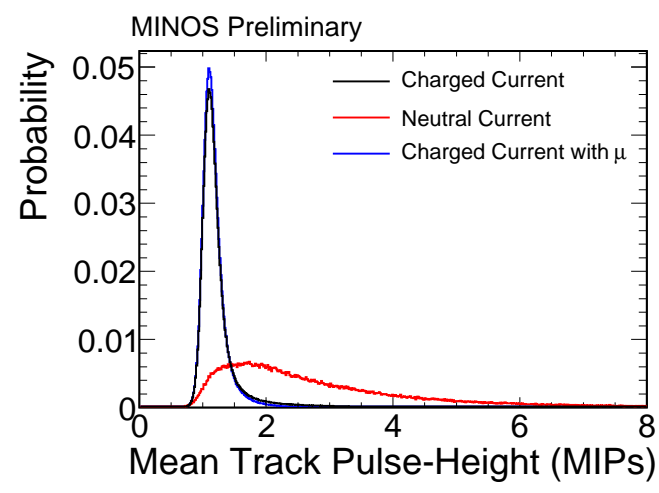

(b) Mean Energy Deposited

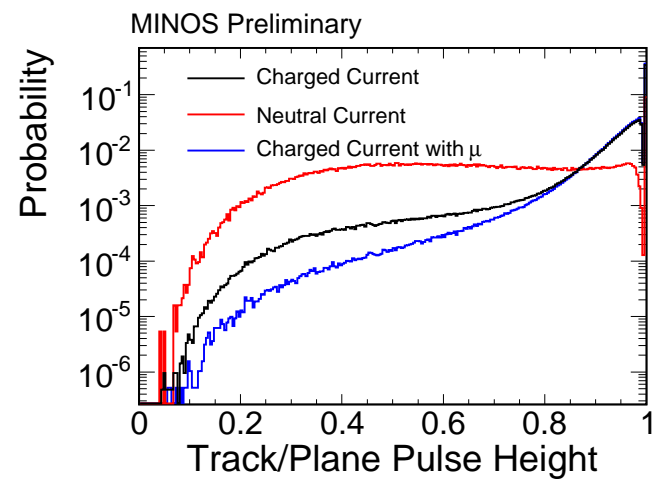

(d) Track Energy / Plane Energy

Figure 4.1: These plots are area normalized distributions of the the minimumionizing discrimination variables in the Near Detector. (a) is the number of active planes in the track (b) is the mean pulse-height in the track after removing the first $20 \%$ in MIPs. These are the discriminating variables which are used in $\mu \mathrm{ID}-\mathrm{A} . \mu \mathrm{ID}-\mathrm{A}$ is the basis of the charged current event selection used in the $2008 \nu_{\mu}$ disappearance analysis. (c) is the ratio of the sum of lower than the median energy deposition to higher than median energy deposition. This describes the fluctuation of energy deposition along the track. (d) is the track to plane variable, which is the ration of energy on the track to energy on the surrounding plane and describes how well separated the track is from the shower. The phrase 'pulse-height' is equivalent to the energy deposited (and seen) per plane. . The red curve shows the neutral current distribution, the blue and black curves show the charged current distribution for all charged current events (black) and those where the muon was found (blue). 
fraction of charged current events from this Monte Carlo "training sample" which have similar values of the four discriminators. Events with a value of the separation variable larger than some defined threshold are labeled as charged current events. MINOS can easily adjust the strictness of the charged current selection by adjusting the separation parameter threshold. A separate set of discriminating variables exists for the Near Detector and Far Detector. Therefore, this algorithm is performed separately for the two detectors. The discriminating variables for Monte Carlo and data are shown in Figure 4.2. For the purposes of clarity and brefity in the text, we call the parameter based on the k-NN separation parameter based on these four discriminating variables the $\mu I D-A$.

$\mu \mathrm{ID}$-A for the Near Detector is shown in Figure 4.3, where it is labeled the "CC/NC separation parameter". This parameter was initially developed by Rustem Ospanov for use in the 2008 MINOS $\nu_{\mu}$ analysis [69]. The remainder of this chapter will describe an anaysis to refine the charged current event identification ability by adding a new separation parameter. In addition to the analysis described in the next section, the variables and $\mu \mathrm{ID}-\mathrm{A}$ described were reevaluated in order to incorporate improvements to the simulation and reconstruction and to keep compatibility with other components of the analysis. This maintainance was part of the work to provide MINOS with a charged current event identification.

\subsection{The Challenges of Muon Identification at Low En- ergy}

Identifying charged current muon neutrino events at low energy suffers

from some interesting statistical problems. First, neutral current events are 


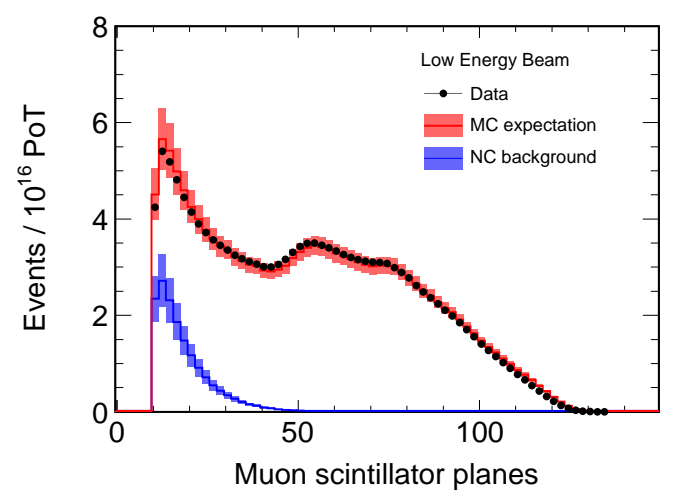

(a) Number of Active Planes

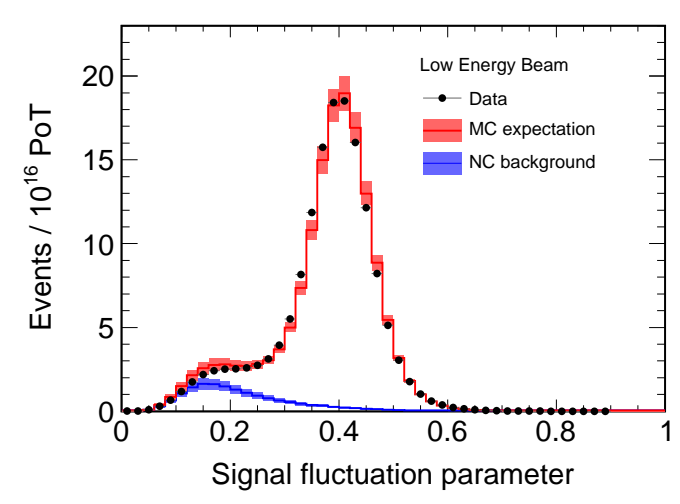

(c) Signal Fluctuation

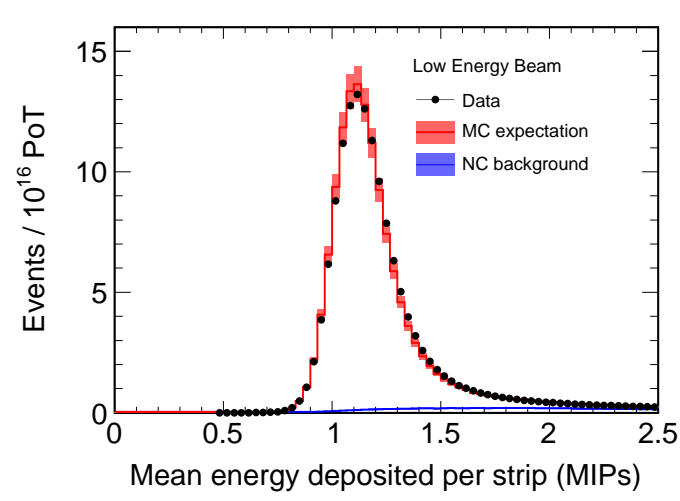

(b) Mean Energy Deposited

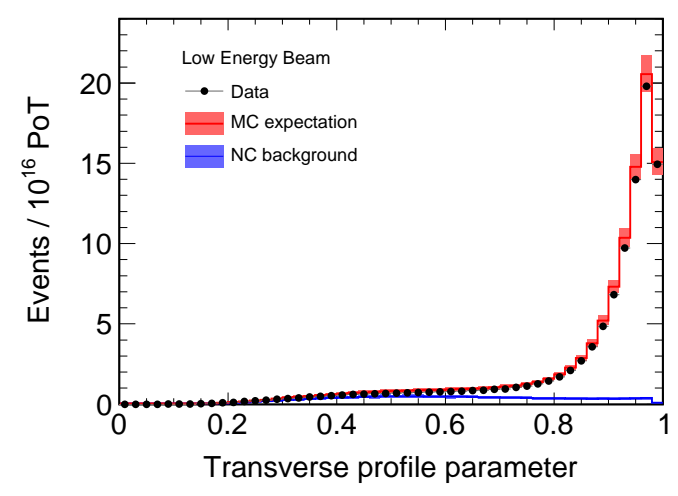

(d) Track Energy / Plane Energy

Figure 4.2: Data to Monte Carlo comparisons for the discriminating variables used in $\mu$ ID-A. The data and Monte Carlo used here are from the LE010185N running. Note that the number of neutral current events is much smaller than charged current events. Generally, the neutral current cross section is lower than the charged current cross section. Additionally, events must contain a track in order to be included in the above histograms. This is a requirement easily satisfied by most charged current events, and less commonly satisfied by neutral current. (d), the "Track Energy/ Plane Energy" is shown on a linear scale here as opposed to the log scale in Figure 4.1. These plots were provided by A. Blake [70]. 


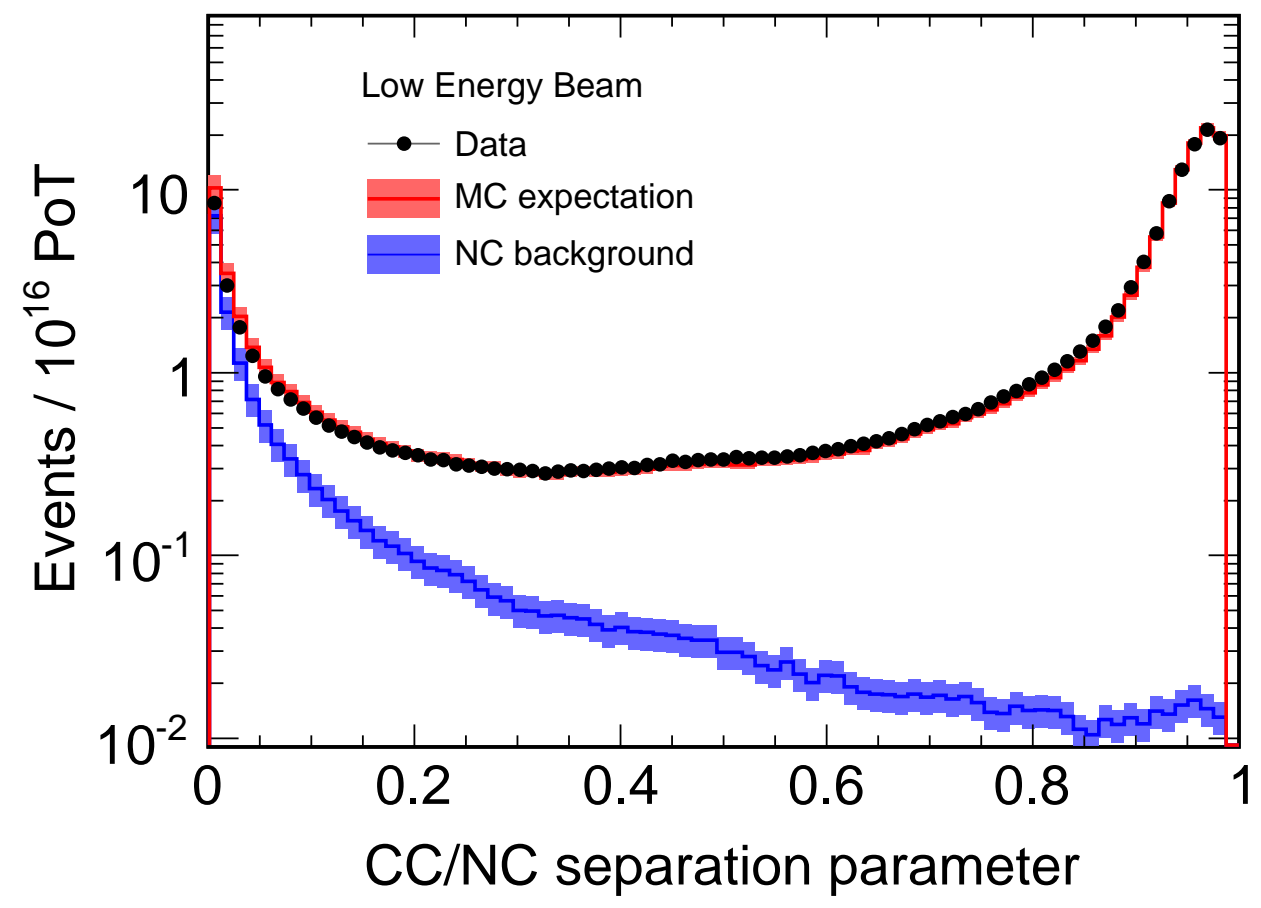

Figure 4.3: $\quad \mu \mathrm{ID}-\mathrm{A}$ or "CC/NC separation parameter for data and Monte Carlo events in the fiducial volume of the ND. This is a k-NN interpolation of the four muon variables shown in Figure 4.2. The input variables for this strong muon identifier are shown area normalized in Figure 4.1. The red curve represents the total Monte Carlo. The blue curve is the neutral current fraction of that Monte Carlo. The black points are the ND data. 
reconstructed at an energy lower than their true energy. This is because the neutrino in the final state of a neutral current event leaves undetected with a fraction of the original neutrino's energy. The hadronic particles that result from a neutral current event have lower energies than most muons and, because they are not minimum ionizing, do not travel far in the MINOS detectors. If a track is reconstructed from the resulting hadronic shower of a neutral current event, the event mimics a lower energy charged current event with a short muon. Although there tend to be more reconstructed charged current events than neutral current events overall, at low reconstructed energies neutral current events dominate.Secondly, at the FD we expect to see even less charged current events at low energies because the maximum deficit is expected to occur in this region. An analysis to increase the sensitivity at low energy will expect to increase the potential to see low energy muon neutrino events at the far detector, but because of oscillations, such events are not expected to be seen. Even with all these difficulties, can we quantify the hand-scan findings and develop new discrimination techniques to help identify muon neutrino charged current events in a high-background environment?

The separation given by $\mu \mathrm{ID}-\mathrm{A}$ in Figure 4.3 is exceptionally good. However, this figure includes events of all energies and its implications of the efficiency can be misleading; high energy charged current events can be easily identified by the long, high-energy muon that is seen in the final state. At lower energies, $\mu \mathrm{ID}$-A becomes more dependant on the three weaker variables. Because of this, the separation of $\mu \mathrm{ID}$-A diminishes at low energies. Resolving the low edge of this dip is essential if one wants to definitively demonstrate the specific functional form for the depletion of events in the energy spectrum observed by MINOS. Variations in this form could indicate the depletion of 
events is due to something other than quantum mechanical oscillations.

The loss of efficiency is compounded by an implicit selection on the event length to form a track, and thus, a charged current event muon track. The last three discriminators in Figure 4.1 are calculated after removing the first $20 \%$ of the track by range in each view. That is, if a track were 100 planes long, only the last 80 planes would be used. Because of this 20/80 rule, there is an implicit requirement that a track must be at least 10 planes long in order to be considered for selection as a muon. A track must have a minimum of five planes for the first $20 \%$ (and no more) to be removed. A 0.3 MIP cut is enforced before the 10-plane selection is made. The total requirement is that there must be 5 planes in each view with at least $0.3 \mathrm{MIP}$ or more energy to be considered a muon.

Figure 4.4 shows the separation variables discussed in Section 4.1 (and used as the input variables to $\mu \mathrm{ID}-\mathrm{A}$ ) for neutrino events with $E_{\nu}^{\text {reco }}>3 \mathrm{GeV}$ and with tracks which are less than 20 planes long. This low energy, short track sample displays a much weaker separation than the variables for all events in Figure 4.1.

Figure 4.5 shows the number of events lost by the selection of charged current events used in the 2008 MINOS $\nu_{\mu}$ analysis as a function of reconstructed neutrino energy for Monte Carlo ND fiducial events. This analysis selected events with a value of $\mu \mathrm{ID}$-A greater than 0.3 as part of the charged current event sample (this is discussed in more detail in the next chapter). Note the dramatic rise at low energy. This motivated a hand-scan of lowenergy Monte Carlo events to attempt to understand how we can better identify low energy muon-neutrino events [71]. This hand-scan found qualitatively that hadronic particles often had a distinguishing large deposition of energy 
at the end of the track (and sometimes along the track). Such large energy depositions are characteristic of capture processes such as $\pi^{-}+p \rightarrow \pi^{0}+n$ or

$K^{-}+p \rightarrow K^{0} n$, which result in a large amount of visible energy from neutral pion decays or neutron capture. An example of such a large energy deposit at the end of a track is shown in Figure 4.6. In contrast, an event with no such deposit is shown in Figure 4.7. This is more characteristic of a low-energy muon ranging out within the detector.

\subsection{Identifiers of Low-Energy Muons}

In order to overcome the shortcomings at low energy of $\mu \mathrm{ID}-\mathrm{A}, \mathrm{MINOS}$ began to emphasize the development of new methods to recover low energy events. The easiest method of including more low-energy events increase the charged current sample at low energy by removing the implicit 10 planes lower limit on track length imposed by the input variables of $\mu \mathrm{ID}-\mathrm{A}$. However relaxing this requirement increases the pool of potential muon neutrino events, it also increases backgrounds substantially. Because the new events would automatically fail $\mu \mathrm{ID}-\mathrm{A}$ due to their length, a new identification variable was necessary to reduce the increased background down to a manageable level.

In this section, we describe the development of discriminating variables for low-energy muon identification. This is indeed a 'needle-in-a-haystack' analysis, where we expect to be overwhelmed by large backgrounds, in search of a couple percent increase in signal strength (which will translate to a few events below $2 \mathrm{GeV}$ ). Because we wish to regain events that fail $\mu \mathrm{ID}-\mathrm{A}$, the variables developed in this section are integrated into a new k-NN based muon 


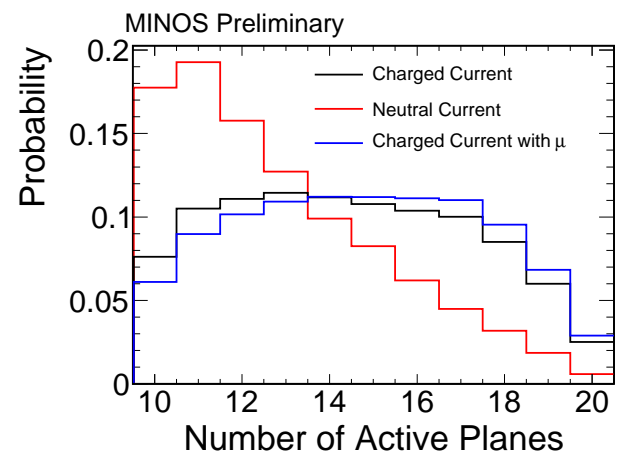

(a) Number of Active Planes

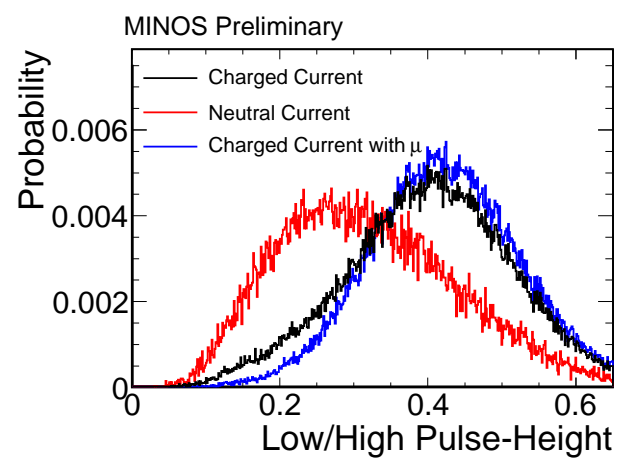

(c) Signal Fluctuation

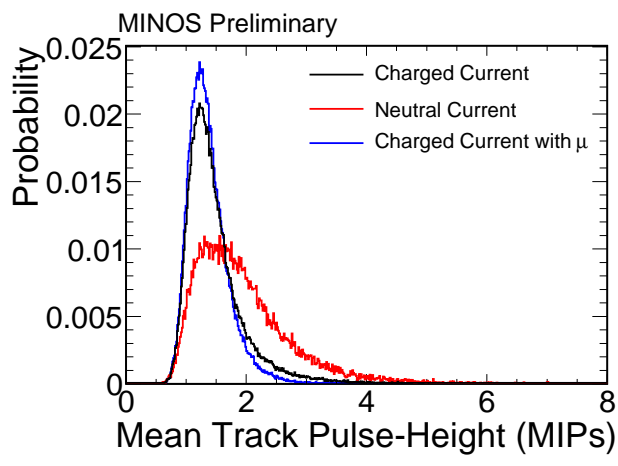

(b) Mean Energy Deposited

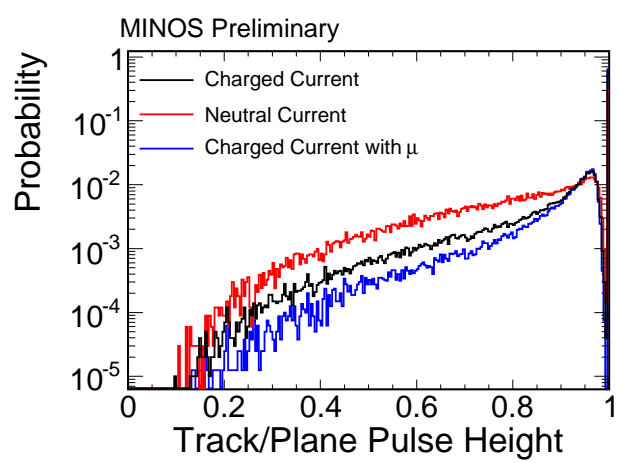

(d) Track Energy/Plane Energy

Figure 4.4: The $\mu \mathrm{ID}-\mathrm{A}$ variables for events with tracks less than 20 planes in length and less than $3 \mathrm{GeV}$ in reconstructed energy. We see that the separation between tracks (muons) from charged current events and tracks (hadrons) from neutral current events is smaller than the separation for all events as shown in Figure 4.1. This is ND monte carlo and data area normalized. 


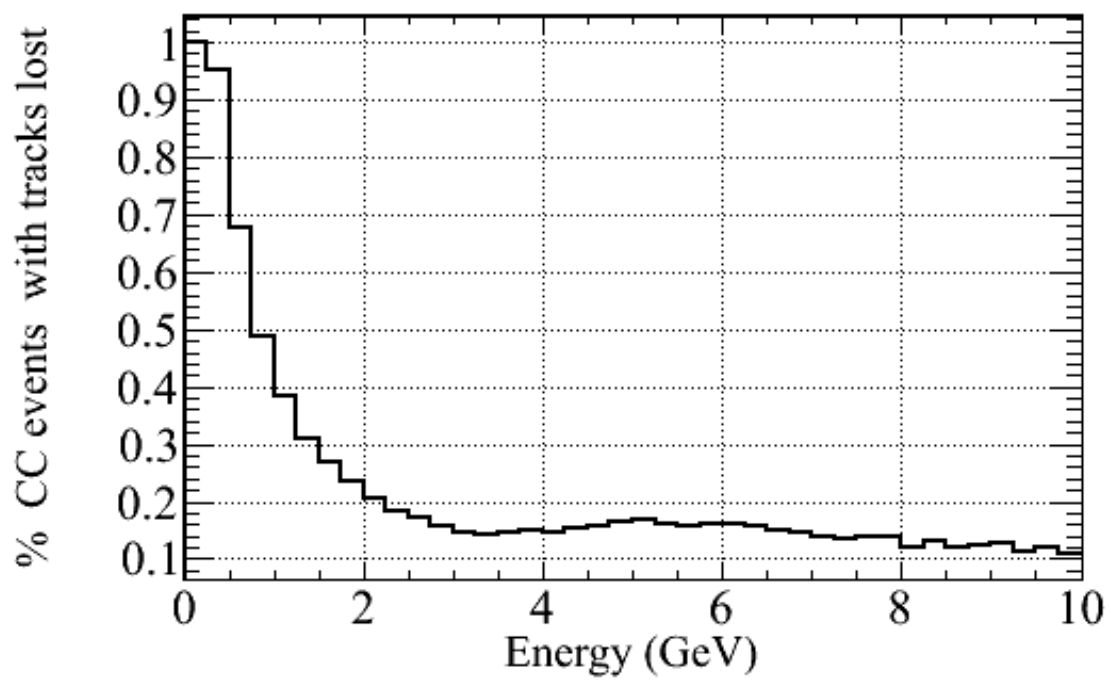

Figure 4.5: Percent of events which have a value of $\mu \mathrm{ID}-\mathrm{A}$ less than 0.3 and are, therfore not selected versus reconstructed neutrino energy in GeV. Note that this this fraction increases below $3 \mathrm{GeV}$, until nearly all muons are lost. 

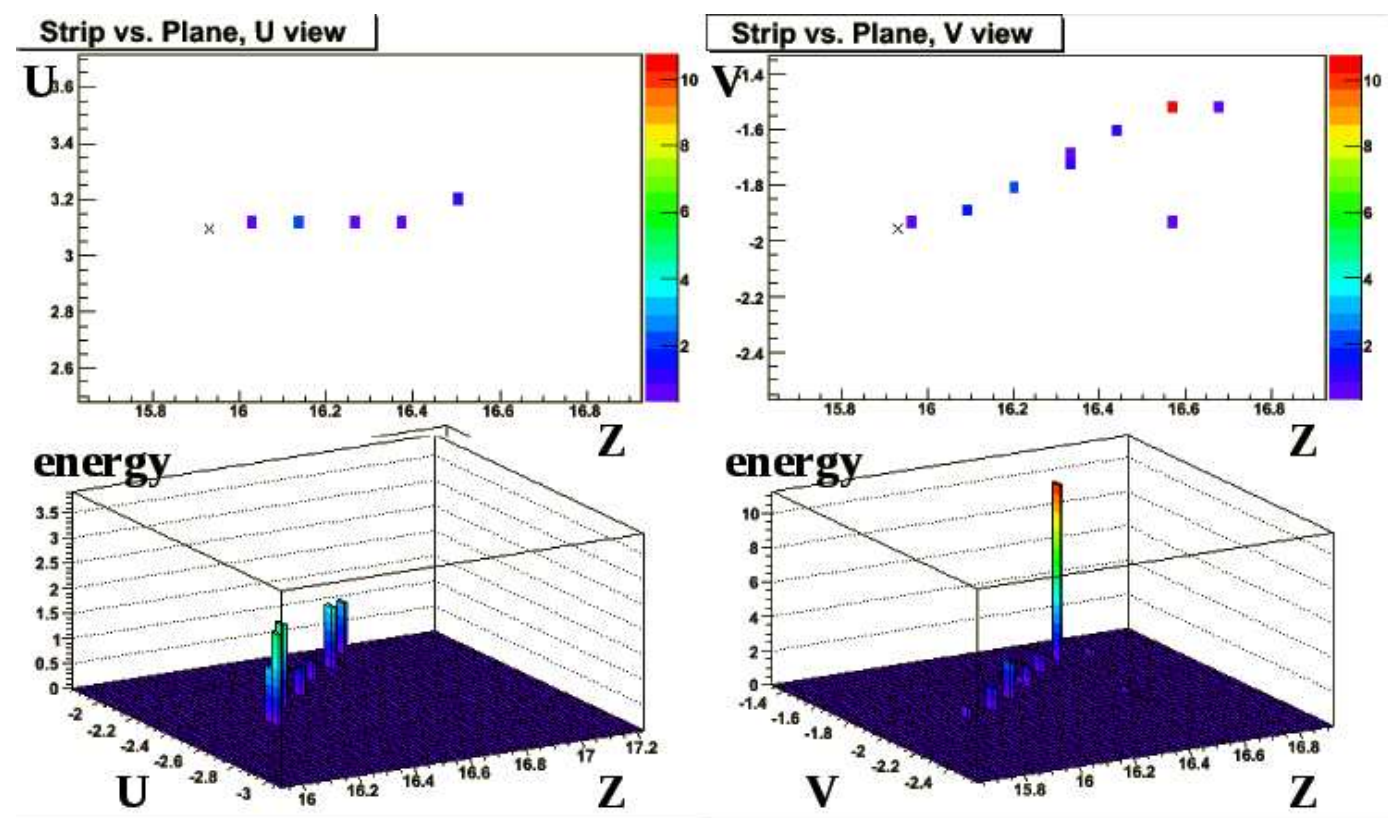

Figure 4.6: A short non-muon track. This is the background to short muon tracks. Notice the large amount of energy lost at the end of the track, indicating non-minimum ionizing energy loss. Energy is given in units of "MEU", which stands for muon energy unit. A single MEU is the average amount of energy lost in the scintillator of one plane by a muon which strikes the plane perpendicularly. This particular event display is from a hand scan of low-energy events [71]. 


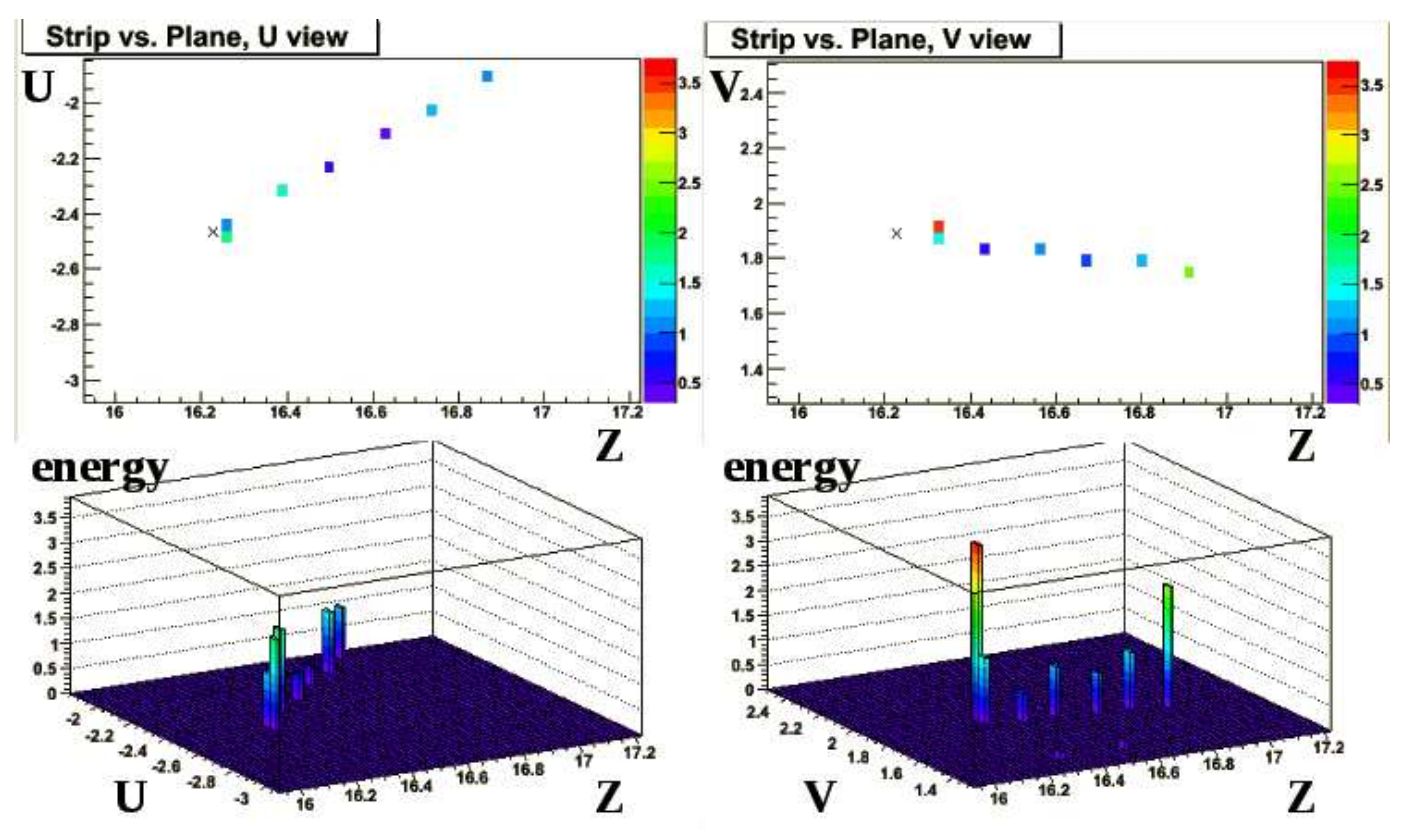

Figure 4.7: A short muon track. We wish to reclaim tracks like this, which are short, yet muon like. Energy is given in units of "MEU", which stands for muon energy unit. A single MEU is the average amount of energy lost in the scintillator of one plane by a muon which strikes the plane perpendicularly. This track has no large deposit of energy. This particular event is from a hand scan of low energy events[71]. 
particle identifier. ${ }^{3}$ This low energy identifier would consider events rejected by $\mu \mathrm{ID}-\mathrm{A}$, giving events a second chance to be included in the charged current event sample. Like the strong variables, these low energy variables would be evaluated separately for the ND and FD, creating a muon identifier which is tailored to each detector.

By removing the 10-planes requirement on track length, the low boundary of the "Number of Active Planes" variable decreased and the range increased. Although the goal of this study was to increase the acceptance at low energy, identifying high-energy muon neutrinos is easily done if one keeps this variable. Even at short track lengths, the number of scintillator planes is a strong identifier; muons are much more likely to have 10 planes than 9. Therefore, the number of active scintillator planes is the first low-energy muon identification variable. The revised active planes variable is shown in Figure 4.8 (a) area normalized and in Figure 4.9 (a) for all events in the fiducial volume of the ND for Monte Carlo and data.

From the hand scan which instigated this study, we qualitatively found that the pion and proton tracks can be distinguished by the energy deposition at the end of the track, characteristic a nuclear interaction. Although a muon's energy loss does increase as it travels towards the low $\beta$ section of the BetheBloch formula, usually the muon stops before a large amount of energy is deposited at the end of the track. The large increases in energy are indicative of a nuclear interaction. To quantify this, we considered the energy in the last five planes of the track. This variable implicitly requires that a track must

\footnotetext{
${ }^{3}$ Additionally, the training sample size increases dramatically for each new 'dimension' of the k-NN feature space, making a 7 or 8 dimensional space harder to manage. Furthermore, the k-NN algorithm does not handle correlated variables well. The features in $\mu \mathrm{ID}-\mathrm{A}$ and $\mu \mathrm{ID}-\mathrm{B}$ are likely to have some correlations.
} 
pass through at least five planes. However, this is already required by the reconstruction; There must be at least 3 'hits' on separate planes in each view for a collection of hits to be considered a track. This new variable is shown in Figure 4.8 (b) area normalized and in Figure 4.9 (b) for all events in the fiducial volume of the ND for Monte Carlo and data.

After another round of hand scanning, we found that a muon's trajectory was relatively straight, only curving because of the magnetic field. In contrast, particles with hadronic interactions have a higher probability to, suddenly move in a new direction. In addition to these physical differences, often track reconstruction can be confused by large hadronic showers. It is not uncommon for short 'tracks' to be a meaningless connection of hits, which are clearly not associated by eye. Such fake tracks are often extremely straight, suggesting that the track finder only connected these hits because of a promising but ultimately meaningless line, or very scattered. Because the U and V strip views of MINOS are completely independent, it is necessary for there to be two variables, one for each view. The k-NN algorithm will be used later to create a single separation parameter. Therefore, it was deemed unnecessary to combine the information of these two scattering variables before this inclusion.

The scattering variable for each view is a function of the pearson coefficient (for the $\mathrm{U} / \mathrm{Z}$ and $\mathrm{V} / \mathrm{Z}$ views), which describes the linearity of a group of points. These consist of two independent variables. A pearson coefficient is defined as

$$
\rho=\frac{\sum_{i} x_{i} z_{i}}{N \sigma_{x} \sigma_{z}}
$$

where $\mathrm{x}$ and $\mathrm{z}$ are the transverse and longitudinal position of the hit ( transverse position is known in one direction, $\mathrm{U}$ or $\mathrm{V}$ only). Unfortunately, the pearson coefficient by itself is not enough to quantify the straightness of the 
track; most tracks are relatively straight, so the differences in "scattered" tracks and "straight" tracks are confined to a relatively small region near a pearson coefficient of one. To magnify these differences, the scattering variable, $\phi$, is

$$
\phi=\frac{0.01}{1.01-\rho}
$$

This quantity is shown in Figure 4.8 area normalized, and in Figure 4.9 (b) for Monte Carlo and data events in the ND fiducial volume for a single view.

In Figure 4.10 the three new variable definitions are shown for events in the near detector where the number of active scintillator planes is less than 20 and the total reconstructed energy is less than $3 \mathrm{GeV}$. Comparing this to the variables in Figure 4.4, it is obvious that the number of active planes in this region has more separation due to the fact that we have allowed the shortest tracks, below 10 planes in length into this sample. Such tracks are mostly from neutral current events, giving the effect of a larger separation. The end of track energy seen in Figure 4.10 (b) has significantly more separation than its comparable variable in the Figure 4.4, the mean energy per plane. Finally the scattering variable has separation at the edges, demonstrating that neutral current events are more likely to have extremely scattered tracks, which have a value near zero, or extremely straight tracks, which have a value near one. Charged current events are more evenly spread, demonstrating that a muon's deviation from a straight line has a different distribution. Although this variable does not have significant separation, it is far better than the track/plane energy ratio shown in Figure 4.4 (d) and is near equivalent to to the signal fluctuation variable Figure 4.4 (c). Furthermore, because two such variables exist in for the two independent views, the separation of the combined variable is much stronger. 


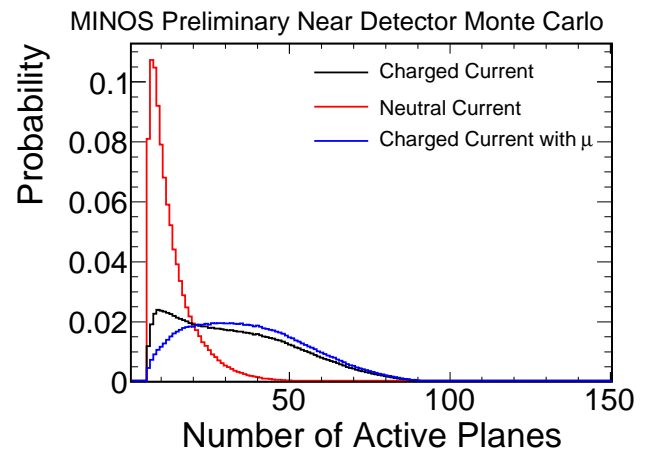

(a) Number of Active Planes (revised)

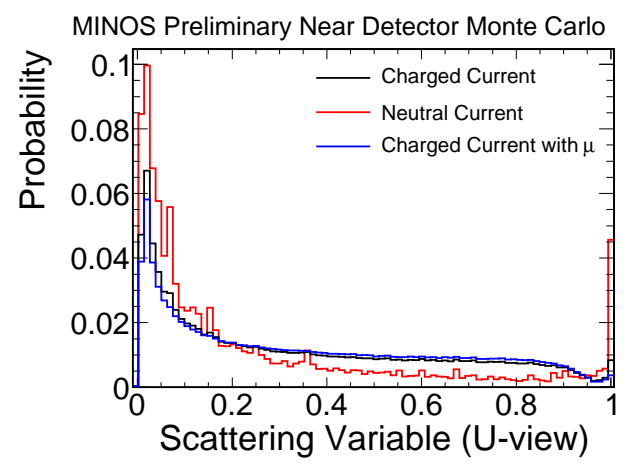

(c) Scattering Variable

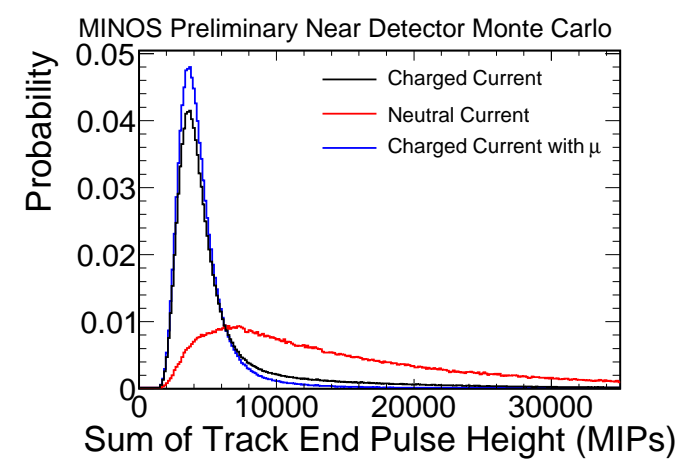

(b) End of Track Energy Deposition

Figure 4.8: The low-energy muon variables. (a) is the number of track planes, including events with less than 10 planes per view. (b) is the energy at the end (last 5 planes) of the the track. (c) is the scattering variable for the $\mathrm{U}$ view. The $\mathrm{V}$ view scattering variable looks identical to this. 


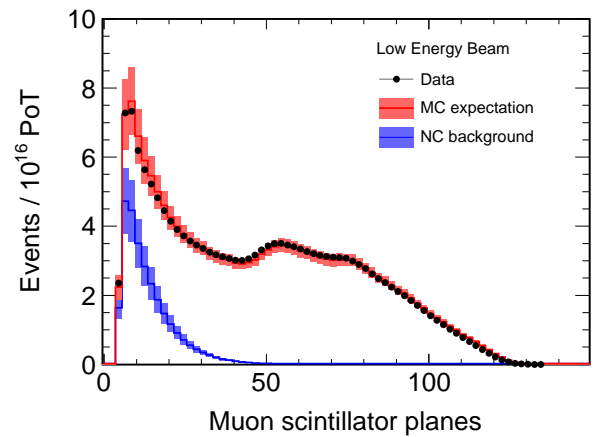

(a) Number of Active Planes (revised)

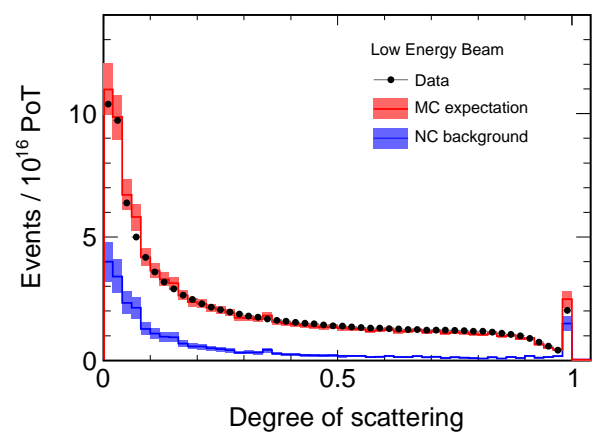

(c) Scattering Variable

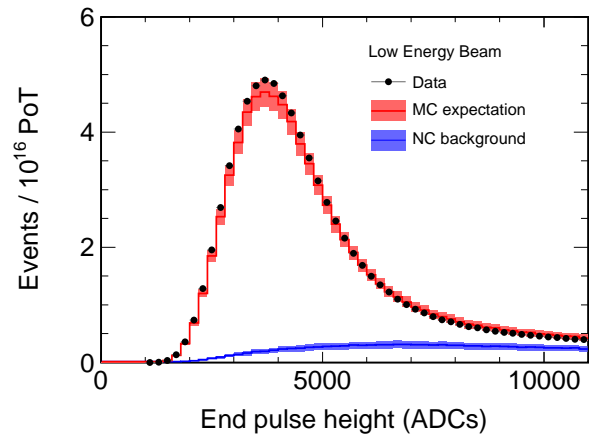

(b) End of Track Energy Deposition

Figure 4.9: The variables developed for low-energy muon identification. The revised number of active planes variables (including all track planes) is shown in (a). (b) is the total ADC count in the last five planes of the track. This is a measure of the energy at the end of the track. (c) is the scattering variable for a single strip direction. There is independent scattering in both strip views of the MINOS detectors. These plots were provided by A. Blake [70]. 


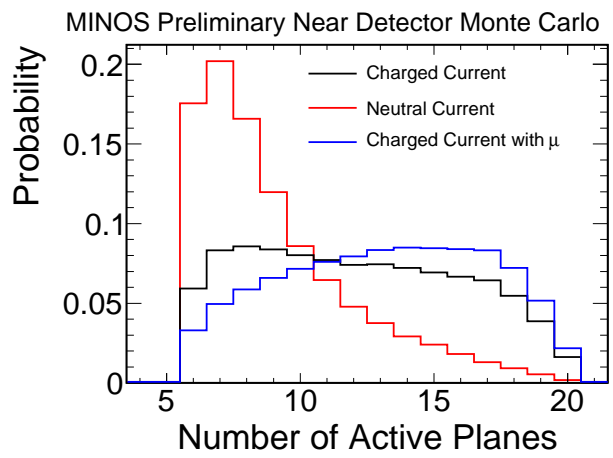

(a) Number of Active Plane(revised)

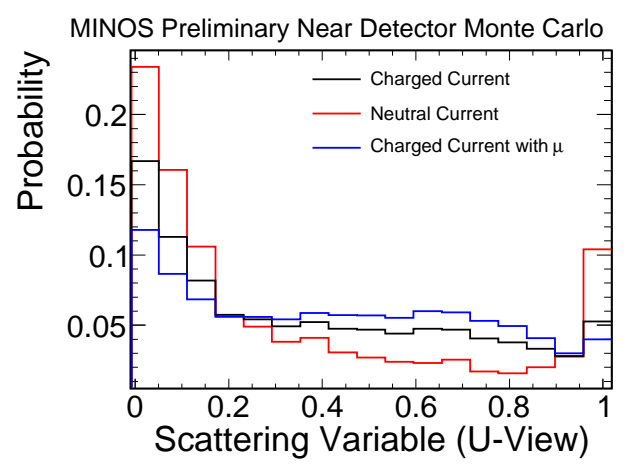

(c) Scattering Variable (U view)

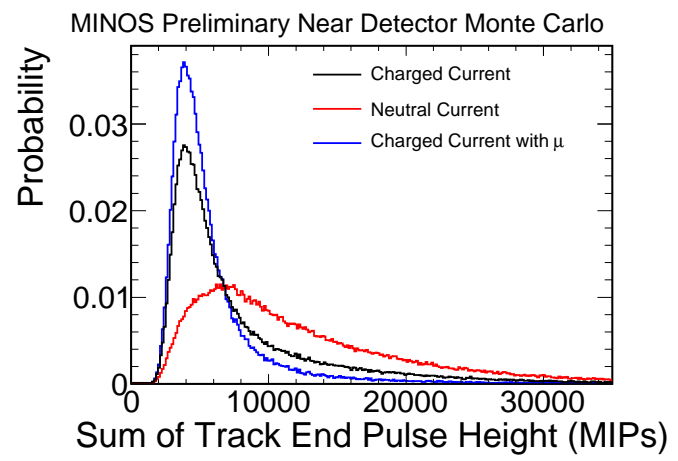

(b) End of Track Energy Deposition

Figure 4.10: The discriminating variables developed for low-energy muons. The events in these plots have less than $3 \mathrm{GeV}$ reconstructed energy and less than 20 planes. The variables shown are (a) the number of active scintillator planes passed by the track, (b) energy deposited at the end of the track in units of ADC, and (c) the scattering variable in the $U$ view. An identical variable exists for the $\mathrm{V}$ view. This is ND Monte Carlo area normalized. 
The "CC/NC separation parameter (low energy)", which in the text shall be called $\mu \mathrm{ID}-\mathrm{B}$, is shown in Figure 4.11 for ND fiducial data and Monte Carlo. This low-energy $\mathrm{CC} / \mathrm{NC}$ separation parameter incorporates the four low-energy variables described in this section (including the two scattering variables) into a single low-energy $\mathrm{k}-\mathrm{NN}$ variable. Together, the prevously developed $\mu \mathrm{ID}-\mathrm{A}$, and the the newly developed $\mu \mathrm{ID}-\mathrm{B}$, can sample more positions in the precision-recall curve (efficiency and purity of the selection) and increase the MINOS charged current selection without increasing the neutral current background to levels which would increase the systematic error of the MINOS-measured oscillation parameters. 


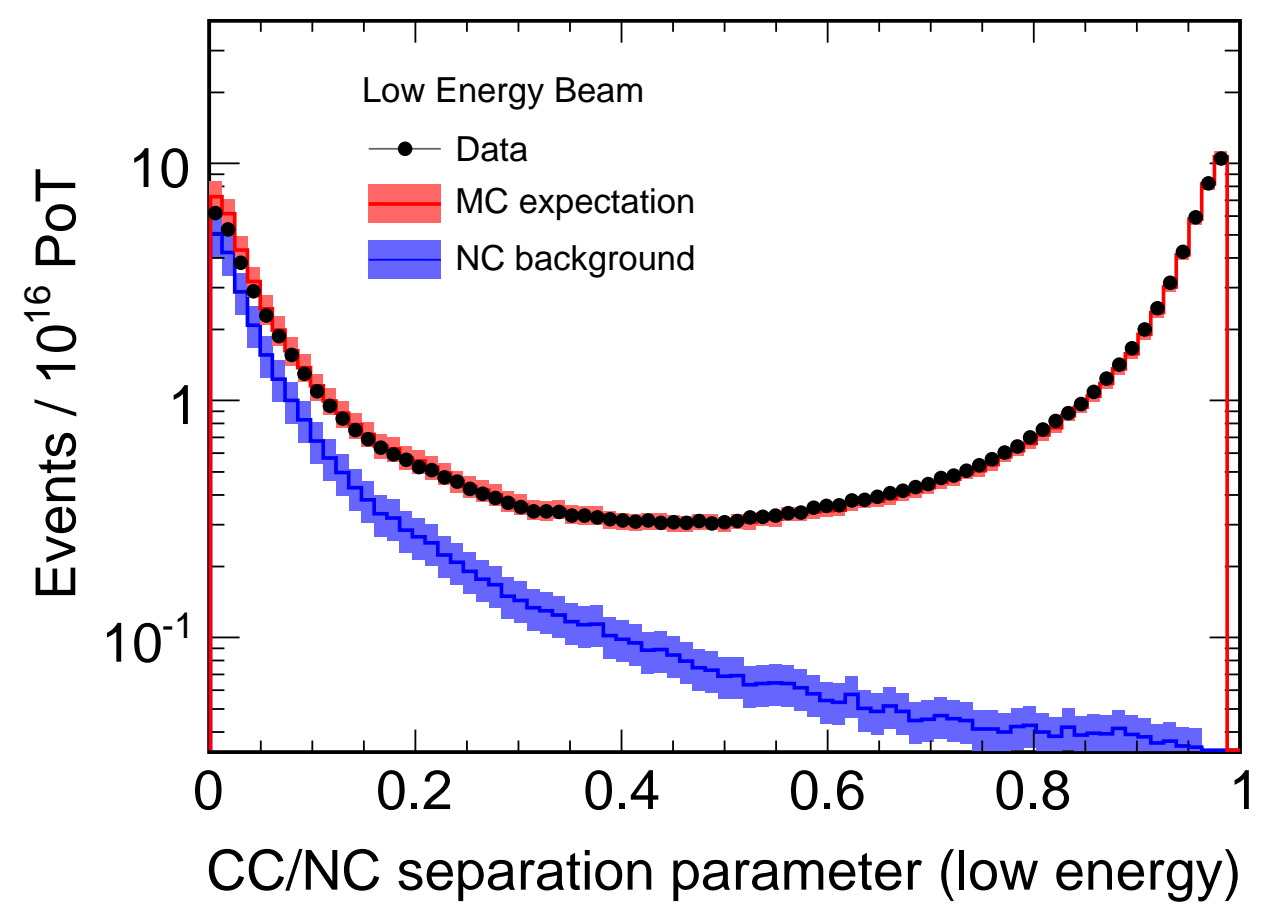

Figure 4.11: $\mu \mathrm{ID}-\mathrm{B}$, or "CC/NC separation parameter (low energy)". This uses a k-NN algorithm to incorporate data from the four low-energy muon variables ( the number of scintillator planes, the end of track pulse-height, and the two scattering variables, one for each view shown in Figure 4.9). 


\section{Chapter 5}

\section{The Neutral Current Background}

Neutral current contamination of the charged current selection is a concern for the MINOS $\nu_{\mu}$ disappearance analysis, as discussed previously in Chapter 4. Although $\mu \mathrm{ID}-\mathrm{A}$ and $\mu \mathrm{ID}-\mathrm{B}$ produce an overall clean selection of charged current events, it is still possible that some neutral current events will be mis-identified by the charged current selection algorithms of Chapter 4 and thus contaminate the charged current sample. This contamination rate increases at low energies and the percent of selected events which are neutral current events rises to nearly $6 \%$ below $2 \mathrm{GeV}$ for the unoscillated sample. The neutral current contamination for $E_{\nu}<2 G e V$ is particularly important because this is the region in which charged current events will have the maximum disapperance through oscillations. Therefore, the neutral current contamination here partially obscures the oscillation effect.

The MINOS $\nu_{\mu}$ disappearance analysis measurements of the oscillation parameters varies the neutral current normalization as part of the fit. Therefore, the neutral current background is accounted for statistically. However, the neutral current background introduces a related systematic error due to uncertainties in Monte Carlo modeling of neutral current events. This systematic error is a primary contributor to the systematic error of the measurement of $\sin ^{2}\left(2 \theta_{32}\right)$, which depends on the depth of the oscillation minimum (see

the discussion in Chapter 1). The main contributions to the neutral current 
mis-modeling are from uncertainties in the neutral current cross section [72], kinematics of Z-boson exchange, hadronization, intra-nuclear modeling, and the modeling of hadron behavior in the MINOS detector. Compounding these general uncertainties, the remnant neutral current background is composed of events from thescattering of high-energy neutrinos, which produce a low multiplicity of particles in the hadronic break-up of the struck nucleus. Such scatters, while rare, are not well understood or measured by current neutrino scattering data. Therefore, neutrino Monte Carlo estimates which are based on these measurements fail to accurately describe the rate and kinematics of such events.

\subsection{Estimating the Neutral Current Background}

The systematic error related to the neutral current contamination must be estimated either by taking uncertainties from different Monte Carlo models or from deriving an uncertainty estimate from data. In light of the large systematic uncertainty that neutral current events cause in the measurement of $\sin ^{2}\left(2 \theta_{32}\right)$, we adopt a data-driven approach [73]. The analysis presented here begins by selecting an identified set of charged current $\nu_{\mu}+\mathrm{Fe} \rightarrow \mu+X$ events and removing the detector hits associated with the muon track. This procedure leaves a hadronic shower of particles recorded in the detector, much as would be expected in a neutral current $\nu_{\mu}+\mathrm{Fe} \rightarrow \nu_{\mu}+X$ scattering event. As shown in Figure 5.1, one can then study the hadronic shower $X$ to record with what frequency a collection of hits in the shower mimics a muon-like track. This procedure, then, utilizes the neutrino data recorded in MINOS to determine a probability that a neutral current event contaminates the charged current sample. 

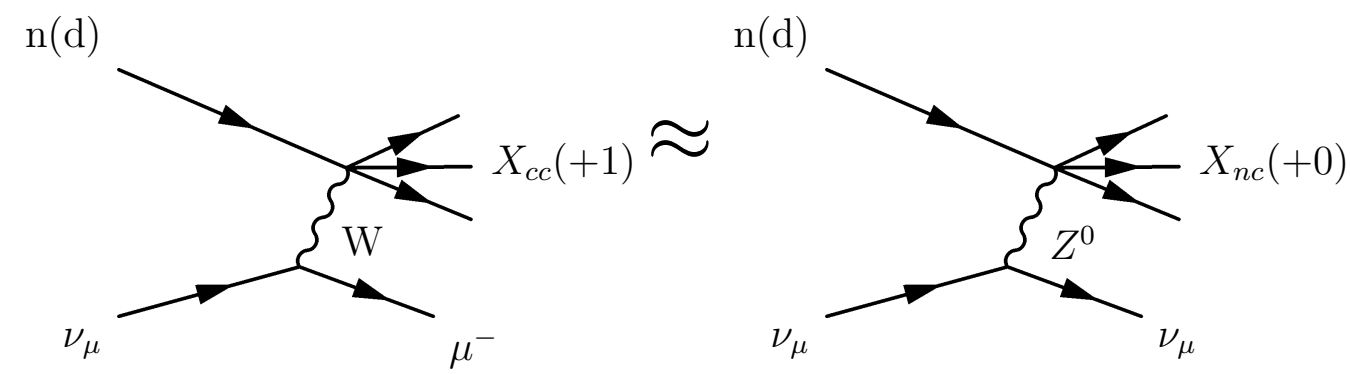

Figure 5.1: Feynman diagrams of a charged current (left) and neutral current (right) neutrino interaction. Removing the $\mu$ from the charged current event reduces it to a hadronic shower, similar to what is seen in a neutral current event. MINOS uses such muon-removed charged current events as a source of hadronic showers, independent of neutral currentcross-sections. This allows MINOS to measure differences in hadronization betweeen data and Monte Carlo.

The procedure, while utilizing the data, assumes that the kinematics of $Z^{0}$ boson exchange and $W^{ \pm}$exchangeare similar. There are some differences, such as the net charge of the shower $X$, which differs by one unit. Monte Carlo modeling of hadronic showers, however, indicates that the hadronic showers are nearly identical for charged current and neutral current events. Because muon-removed charged current events (abbreviated MRCC) are derived from charged current events, they can be derived from both data and Monte Carlo. This allows us to study any residual differences in the Monte Carlo and data hadronic shower modeling and apply such differences as corrections to the data-driven estimate of shower modeling of neutral current events. Thus, by comparing muon-removed charged current events in data and Monte Carlo one can infer valuable information about the mis-modeling of showers in the Monte Carlo charged current events and, through similarity, neutral current events.

The neutral current background distribution can be weighting by the 
muon-removed charged current data/Monte Carlo ratio:

$$
N C_{\text {data }} \approx N C_{\text {MonteCarlo }} \times \frac{M R C C_{\text {data }}}{M R C C_{\text {MonteCarlo }}}
$$

where $N C_{\text {data }}$ is the neutral current data distribution, $N C_{\text {MonteCarlo }}$ is the neutral current Monte Carlo distribution, $M R C C_{\text {data }}$ is the muon-removed charged current data distribution and $M R C C_{\text {MonteCarlo }}$ is the muon-removed charged current Monte Carlo distribution. By correcting for mis-modeling, this ratio is likely to give an estimate of the neutral current background in data which is closer to the true neutral current background. The range of the $M R C C_{\text {data }} / M R C C_{\text {MonteCarlo }}$ ratio is an approximation of how much the Monte Carlo hadronic modeling differs from the true distribution.

The above procedure provides only a probability that a neutral current event will fake a charged current event. An independent estimate must be made of the absolute number of neutral current events in the MINOS $\nu_{\mu}$ charged current sample. This absolute normalization depends on the charged current and neutral current cross sections and the rate at which muons versus hadronic particles are tracked. These values are not solely addressed by the mis-modeling of showers. Furthermore, the number of muon-removed charged current events per POT depends on the hadronic energy distribution of charged current events. Therefore, the muon-removed charged current analysis does not address cross section and track reconstruction uncertainties and their effect on the neutral current background normalization. This is accomplished by comparing Near Detector data and Monte Carlo particle identification distributions ( the $\mu \mathrm{ID}-\mathrm{A}$ and $\mu \mathrm{ID}-\mathrm{B}$ ). The Monte Carlo is divided into it charged current and neutral current distributions and the normalization of these distributions is varied such that a best-fit between Near Detector data and Monte 
Carlo is achieved. Assuming that all the differences between data and Monte Carlo are attributable to normalization uncertainties, the best fit indicates the amount the neutral current normalization must change to achieve a best-fit to data.

There is significant evidence that the Monte Carlo overestimates the neutral current contamination of the charged current selection [33, 73]. In particular, distributions with significant separation between charged current and neutral current events appear to have more separation in data than in Monte Carlo. For example, the separation variables shown in the 2008 MINOS Physical Review D. publication ([33], Figure 22), or Figure 4.3 and Figure 4.11 of this thesis. In the analysis detailed in the 2008 MINOS Physical Review D. publication[33], a direct fit of the data suggests that the neutral current background is actually $37 \%$ smaller for than predicted by the Monte Carlo (as seen in Table VI of the 2008 MINOS PRD [33]). For this same analysis, the neutral current background systematic error was set at $50 \%$. This error was derived from an analysis which used muon-removed charged current events as an alternate source of the neutral current like events. This fake neutral current background along with the expected charged current signal was used to fit the data by varying the relative normalization of the two distributions. The scaled muon-removed charged current distribution and nominal neutral current Monte Carlo distribution had a maximal difference between 0 and 4 GeV of 0.503 (at 1-2 GeV). This suggested that the neutral current background could be reduced by $50 \%$ in this region without affecting fitting of the charged current signal.

An improved study of the neutral current background using muonremoved charged current events was pioneered by John Marshall [73, 74]. 


\begin{tabular}{c|c} 
Reco $E_{\nu} / \mathrm{GeV}$ & neutral current Monte Carlo Corrections (Eff \& Norm) \\
\hline $0-1$ & $-26.7 \% \pm 15.8 \%$ \\
$1-2$ & $0.8 \% \pm 8.8 \%$ \\
$2-4$ & $2.9 \% \pm 7.4 \%$ \\
$4-6$ & $-14.3 \% \pm 10.6 \%$ \\
$6-12$ & $-9.0 \% \pm 9.0 \%$ \\
$12-100$ & $-11.3 \% \pm 10.6$ \\
\hline
\end{tabular}

Table 5.1: The results from a previous study by John Marshall. Adding the corrections in quadrature,a systematic uncertainty of $25 \%$ is reached

These studies were completed with both a predecessor particle ID and $\mu$ ID-A. Marshall computed the ratio given in equation (5.1) to estimate corrections to the 'efficiency' of neutral current events in the charged current selection. Marshall then performed a normalization study, fitting the neutral current background integral to produce the best fit with data and Monte Carlo. A more appropriate systematic error was found using this technique. This error is the quadrature the mean difference in the muon-removed charged current ratio study (20\%) and the error in the normalization study (10\%). The total error was given as $25 \%$. The results from Marshall's studies are summarized as follows in Table 5.1, which shows the efficiency and normalization corrections to the neutral current Monte Carlo in energy bins.

Marshall's study was performed before $\mu$ ID-B had been developed and improvements to the muon removal algorithm and event reconstruction has been integrated. Additionally, Marshall's study only divided the information into energy bins, and not by inelasticity, $(\mathbf{y})$, defined as $\mathbf{y}=E_{h a d} / E_{\nu}=$ $1-\left(E_{\mu} / E_{\nu}\right)$. Inelasticity is an important quantity for the muon-removed charged current analysis because differences in the inelasticity distribution between muon-removed charged current events and neutral current events could 
be caused by the differences in exchanging a $W^{ \pm}$or $Z^{0}$ boson. Furthermore, differences in the inelasticity distribution between muon-removed charged current data and muon-removed charged current Monte Carlo could indicate differences in hadron formation. In distributions of the raw hadronic energy, these effects are convoluted with the neutrino energy, whose effects dominate. This chapter outlines an updated version of Marshall's studyimproved reconstruction, Monte Carlo and muon removal algorithms.

\subsection{Muon Removal Procedure}

The muon removal analysis proceedure is used to create a data and Monte Carlo samples of hadronic showers independent of the simulation [7577]. ${ }^{1}$ The muon track removal process seeks to remove all of the muon energy depositions from the event, while keeping the hadronic shower in tact. A new muon removed list of hits ${ }^{2}$ is created from the remaining energy in the event. The first action of the muon removal algorithm is to identify a maximum shower plane, which is defined as the plane 6 planes away from the track vertex. Any information after this point is not included. The remaining strip information in the event is examined. Strips not included in a track are automatically kept. If a strip was part of a track in the original event, it is examined more carefully to determine whether its energy should be included. If the track is determined not to be a muon, its parent digits are retained for the next reconstruction pass. Otherwise, the energy in the strip is reduced by $(1-1 /(Q \cos (\theta)$ if it has more than 1.2 minimum ionizing particle (MIP) units

\footnotetext{
${ }^{1}$ This muon removal technique is used extensively by other MINOS analyses. In particular, the $\nu_{\mu} \rightarrow \nu_{e}$ analysis uses a "Muon Removed charged current with simulated electron" sample (MRE) as an independent sample of electron neutrino-like events [78-80].

${ }^{2}$ In MINOS, this is called the DigitList.
} 
of energy. The event information, without the energy assumed to be from the muon, is reprocessed with the same reconstruction used for regular data and Monte Carlo.

In Figure 5.2, a Monte Carlo charged current event is shown before muon removal. The same event after the muon removal algorithm is shown in Figure 5.3. In both figures, two plots show the hits (bottom) and the energy(top) which is removed from each event. The red colored hits are hits which have been included in a track object. Notice that a track is found in Figure 5.3 after muon removal even though there was no second track found in the original event. This track is not composed from the same energy deposits as the muon in the original event. After the track removal, the track reconstruction algorithm has found an independent track within the shower. This track could be a random collection of hits, or an actual hadronic particle. This is evidence of the tracking algorithm's dependence on the entire event topology.

In order to compare the muon-removed charged current and neutral current distributions, they must be scaled to the same exposure of the NuMI beam (given in POT, or protons on target). The POT of the muon-removed charged current data and Monte Carlo files are given in Table 5.2. In practice, the POT count before muon removal is much higher than after muon removal (which is reasonable, since there are more events total). The flux reweighting gives rise to fluctuations in the data/Monte Carlo ratio. However, overall, the distributions for using POT counting and weights before and after muon removal are very similar.

Figure 5.4 shows the distribution of reconstructed neutrino energy, $E_{\nu}$, and inelasticity, $\mathbf{y}$, for a set of events which have a reconstructed track af- 

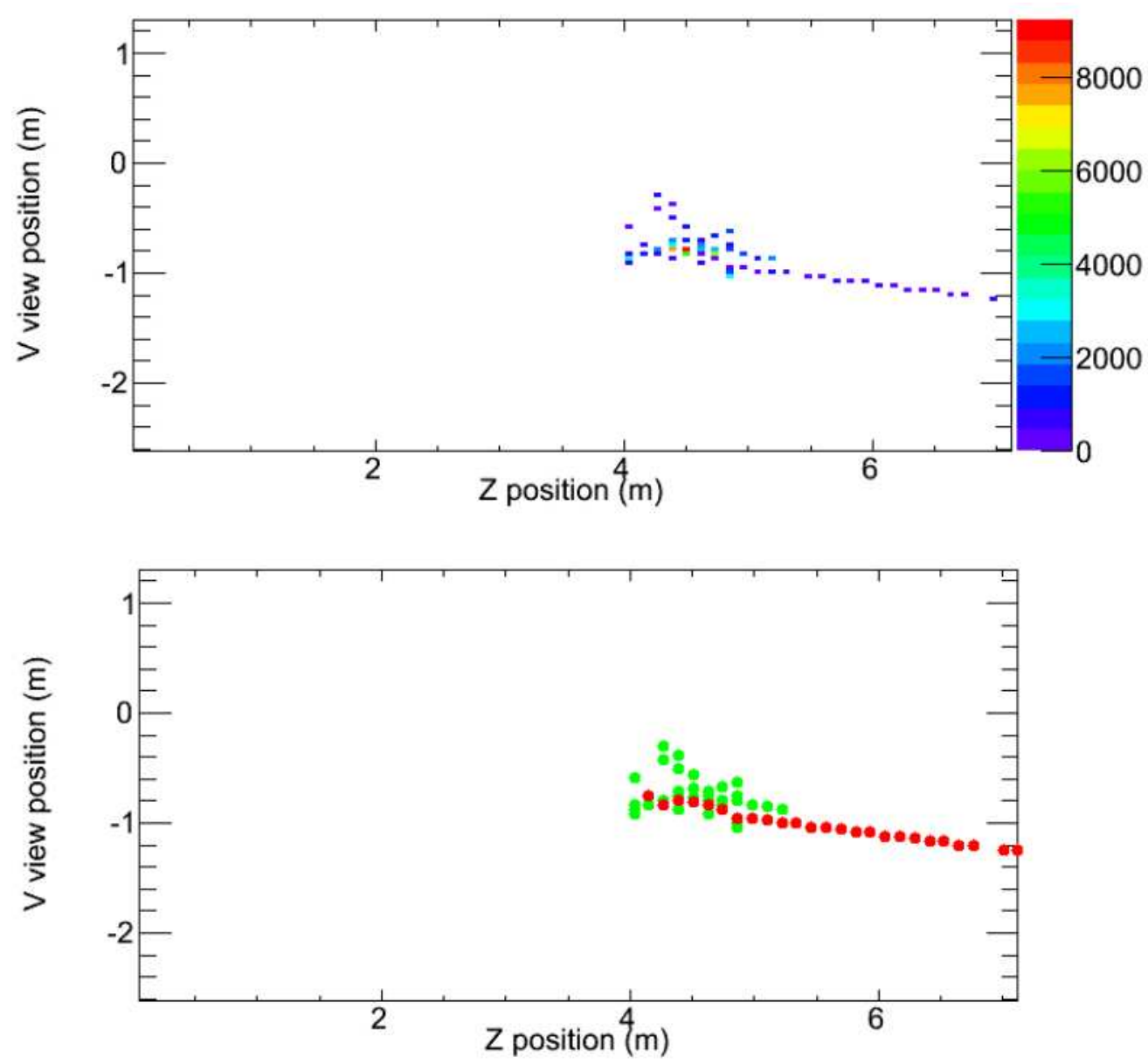

Figure 5.2: A muon event after is shown in the $\mathrm{V}-\mathrm{Z}$ view. The top graph indicates the ADC deposited at each hit. The bottom graph depicts the track (red) and shower (green) hits within the event 

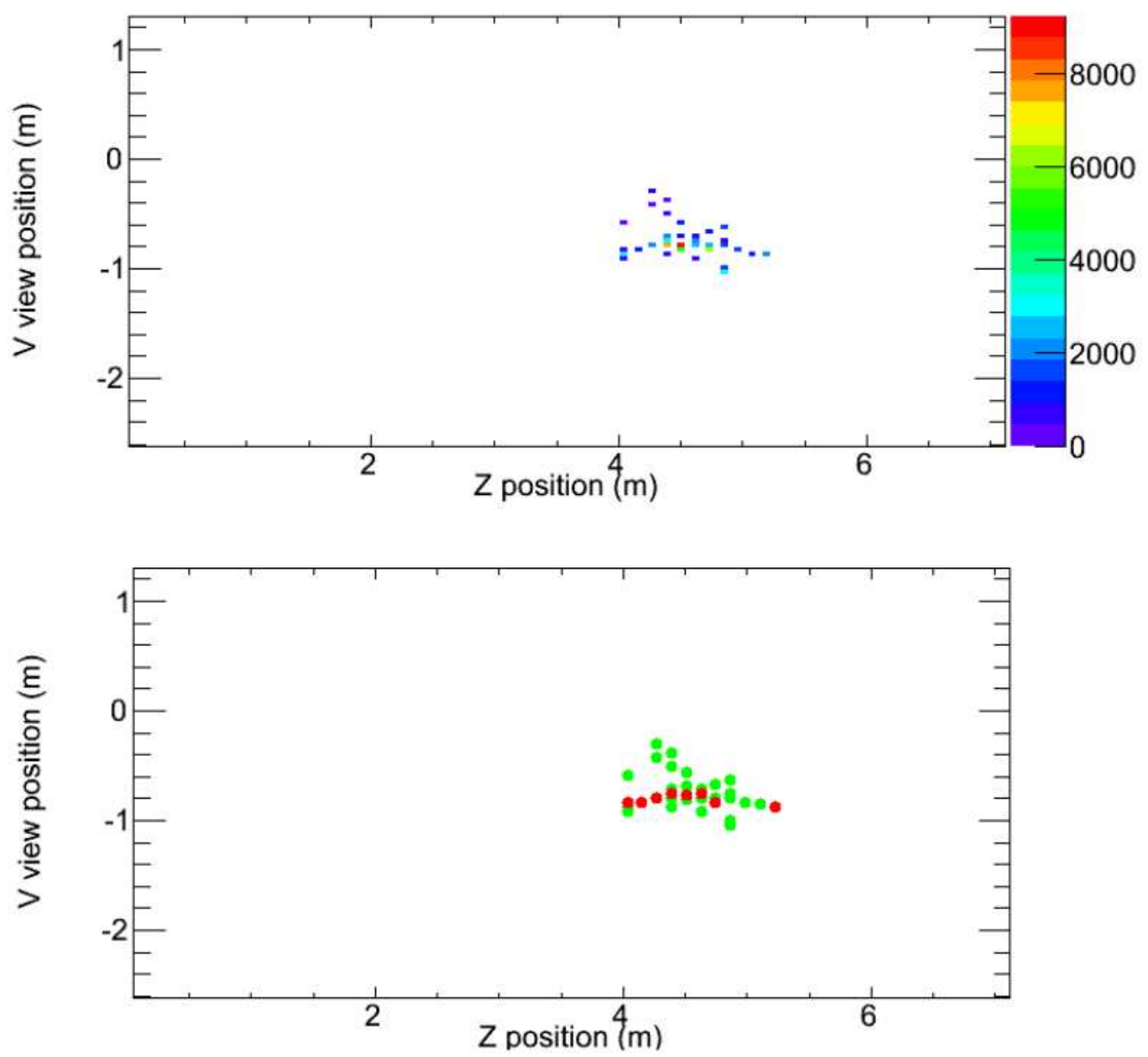

Figure 5.3: A muon event after is shown in the $\mathrm{V}-\mathrm{Z}$ view. The top graph indicates the ADC deposited at each hit. The bottom graph depicts the track (red) and shower (green) hits within the event 


\begin{tabular}{|l|l|l|l|}
\hline & Muon-Removed POT & Original POT & Ratio \\
\hline Monte Carlo & $3.51 \times 10^{19}$ & $12.06 \times 10^{19}$ & 0.29 \\
data & $4.8 \times 10^{19}$ & $15.34 \times 10^{19}$ & 0.31 \\
\hline data/Monte Carlo & 1.38 & 1.27 & 1.08 \\
\hline
\end{tabular}

Table 5.2: A table which shows the difference in the POT count from the original non-muon-removed files to the muon removed files

ter the event has been stripped of its initial muon. That is, in events such as Figure 5.3, the track reconstruction algorithm is re-executed after muonremoval. For the events in Figure 5.3, a second track-like collection of hits is found. The energy and inelasticity are recalculated. The 'muon' in this event is a forged from the hadronic shower of the event in Figure 5.2. Overall, the agreement between data and Monte Carlo is very good except at low energies, where the Monte Carlo over-predicts data, and at high inelasticities, where the Monte Carlo under-predicts data. Note that while a track has been found and these distributions represent an independent source of hadronic showers with reconstructed tracks, not all of the events in these distributions are necessarily background to the charged current signal. That is, these events still need to pass the charged current selection described in Chapter 6.

A question that must be answered before we continue is whether to use the POT and beam weights calculated before or after muon removal. Using the POT weighting before muon removal introduces a subtle dependence on the neutral current and charged current kinematics. However, using the beam weights calculated after muon removal is incorrect because the event should be weighted by the original event structure. In Figure 5.5 the differences in using the POT counting and flux reweighting before and after muon removal are shown. Each entry is the inelasticity of the 'parent' of a muon-removed event 


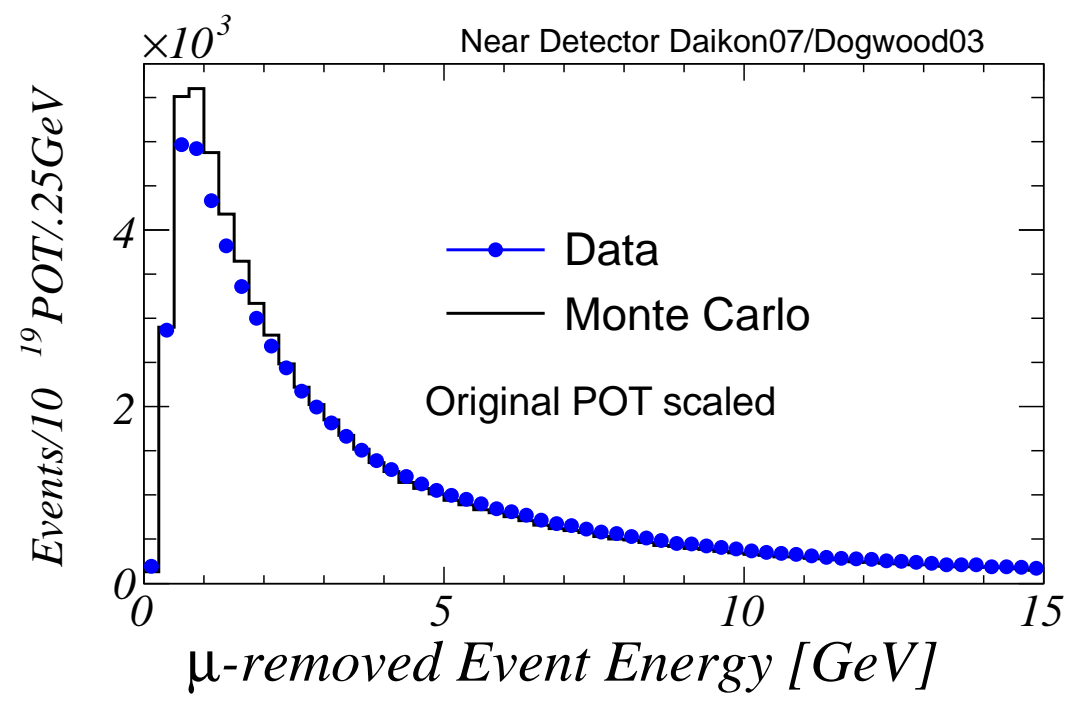

(a) Reconstructed neutrino energy

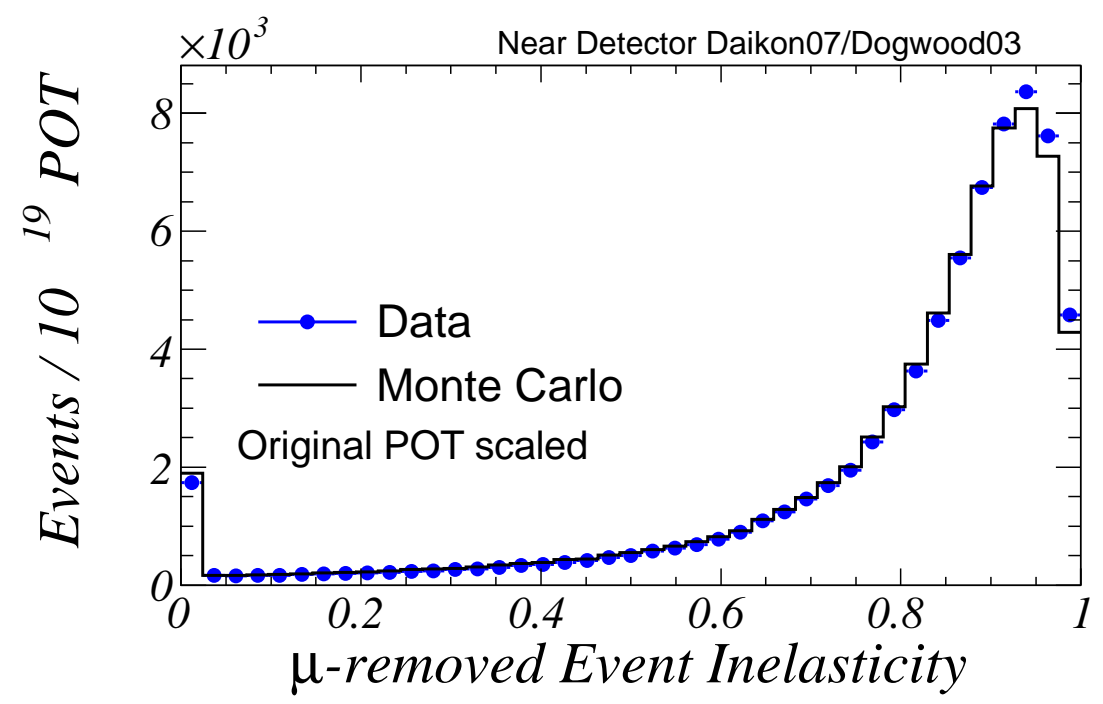

(b) Reconstructed inelasticity

Figure 5.4: The (a) reconstructed neutrino energy and (b) inelasticity for data and Monte Carlo muon-removed charged current events. That is, the original event was found to have a muon-like track. This track is stripped from the event and the event is reexposed to the track reconstruction algorithm and a new track is reconstructed. These muon-removed events are a source of hadronic showers independent of the neutral currentcross-sections. If the event without the muon is reconstructed to be charged current like, it represents a background to the true-charged current signal, which seeks to identify muons (di-muon events are very rare). 
(ratios of the data and Monte Carlo are shown in the bottom two subplots). The middle two plots show the parent event inelasticity after a $\mu \mathrm{ID}-\mathrm{A}>0.3$ selection. The data and Monte Carlo agreement is relatively unchanged. As it turns out, POT scaling does not matter because the neutral current systematic error estimate into two separate analyses: a normalization analysis and a study of the neutral current data/Monte Carlo ratio's dependence on energy and inelasticity. The normalization study does not use muon-removed charged current events. In the muon-removed charged current event studies, only the shape of the distribution is important. The beam re-weighting before and after muon removal does matter. In Figure 5.5, it is apparent that the beam re-weighting changes the Monte Carlo distribution, leading to variability in the data/Monte Carlo ratios. Therefore, using the POT scaling and beam re-weighting for the original events is what is used for the studies discussed in the rest of this chapter.

\subsection{The Neutral Current Background Shape}

The previous analyses of neutral current background estimates using muon-removed charged current events only considered comparing neutral current and muon-removed charged current events in bins of energy. While satisfactory, this method integrates over the more important quantity, the inelasticity. The inelasticity probes what fraction of the energy is given to the

hadronic shower. It will become apparent that the differences between data and Monte Carlo in muon-removed charged current events exhibits a stronger linear dependence with inelasticity than with energy. A more through analyses would divide the distributions of neutral current and muon-removed charged current events both by energy and by inelasticity, such that these differences 


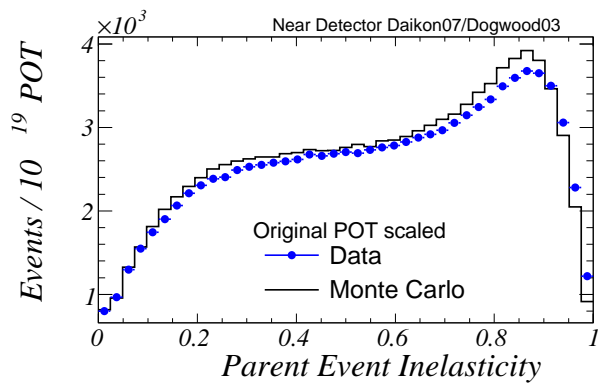

(a) Before muon removal POT counting

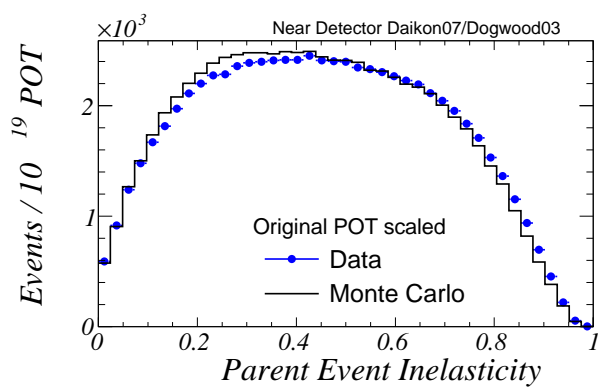

(c) Same as (a) for $\mu \mathrm{ID}-\mathrm{A}>0.3$

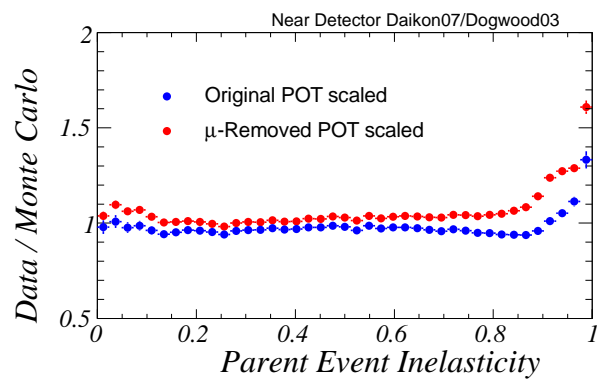

(e) data/Monte Carlo for (a) and (b)

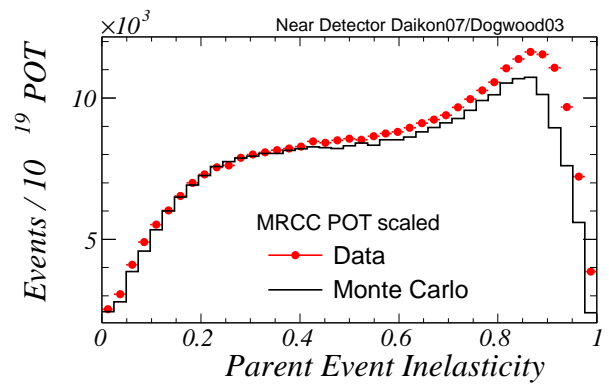

(b) After muon removal POT counting

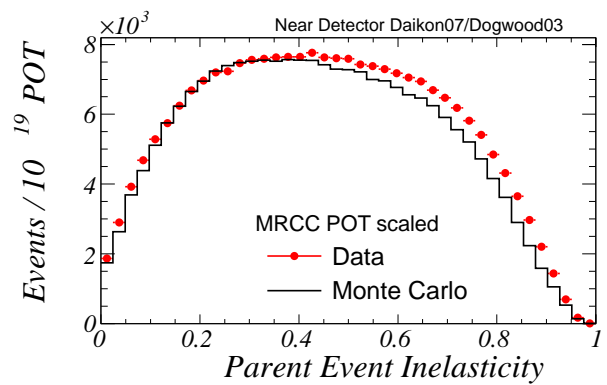

(d) Same as (b) for $\mu$ ID-A $>0.3$

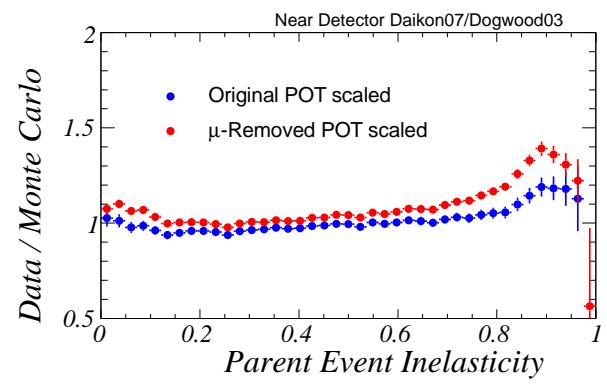

(f) data/Monte Carlo for (c) and (d)

Figure 5.5: The inelasticity of the parent event in data and Monte Carlo (reweighted flux) using the (a) the POT counting before muon removal and (b) the POT counting after muon removal, and the same after selecting reconstructed parent charged current events (parent $\mu \mathrm{ID}-\mathrm{A}>0.3$ ) in (c) and (d) respectively. In (e) and (f) the ratio of data and Monte Carlo are shown before (e) and after(f) the charged current selection. The differences in beam re-weighting (before or after the muon removal) are responsible for the shifts in the shape. Whereas the overall POT normalization adjusts the overall data to Monte Carlo ratio. 

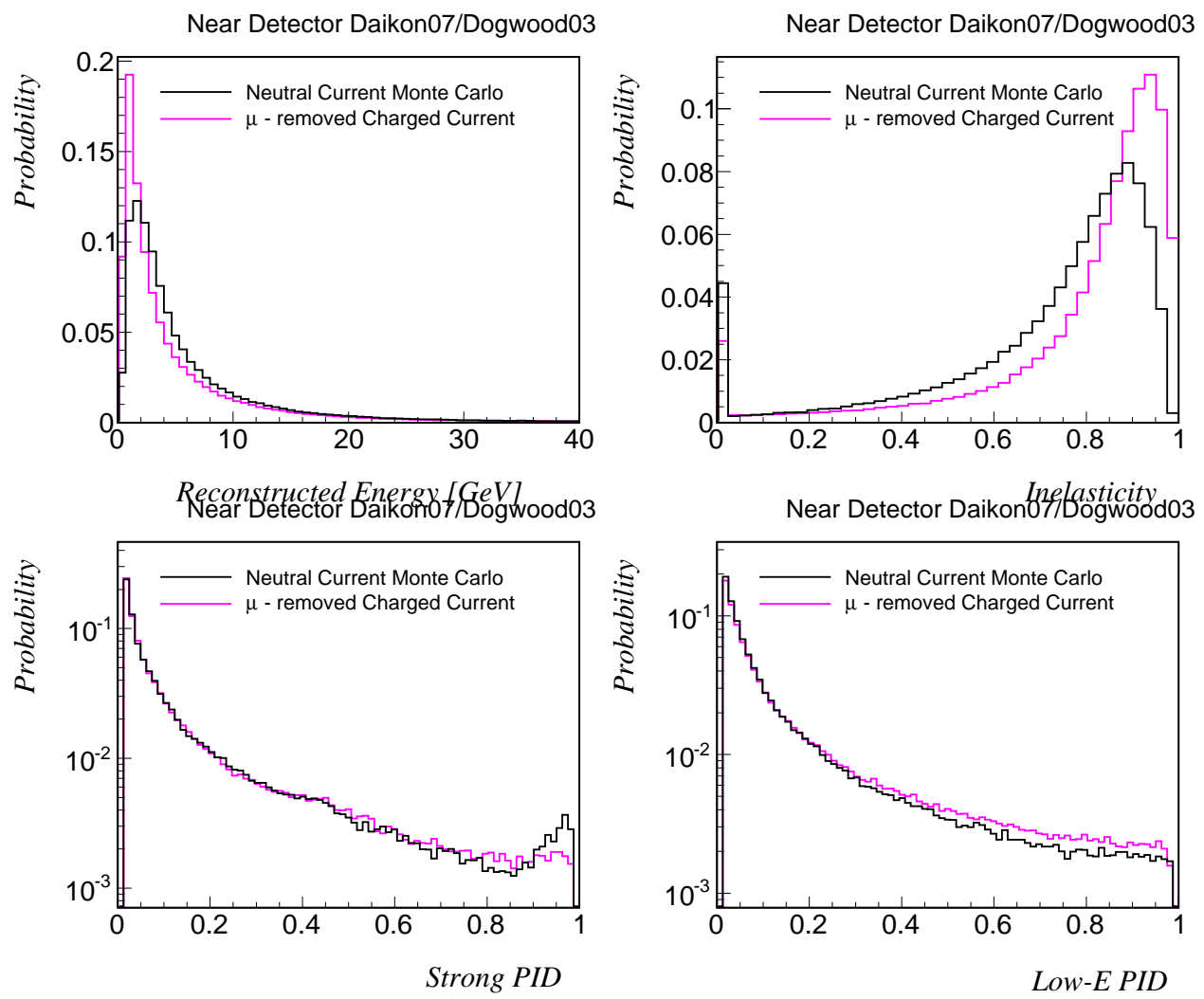

Figure 5.6: Various distributions for muon-removed charged current events derived from events with $\mu \mathrm{ID}-\mathrm{A}>0.3$ and true Monte Carlo neutral current events. These distributions include the (a) reconstructed neutrino energy, (b) reconstructed inelasticity, (c) $\mu \mathrm{ID}-\mathrm{A}$, and (d) $\mu \mathrm{ID}-\mathrm{B}$.

can be incorporated and a better weighting of the neutral current distribution can be obtained. This is accomplished in one of the following analyses.

In Figure 5.6 the kinematic and particle-ID distributions (area normalized) are shown for muon-removed charged current events where the original event has a $\mu \mathrm{ID}$-A greater than the threshold given in Figure $6.1\left(\Theta_{\text {strong }}=0.3\right)$ and true Monte Carlo neutral current events. It is apparent that there are some broad differences in the muon-removed charged current and neutral current distributions. 
For comparison with Figure 5.6, the neutral current and muon removed charged current events which pass the thresholds for selection are compared in Figure 5.7. The neutral current and muon-removed charged current energy and inelasticity distributions are similar in Monte Carlo. Although the distributions in Figure 5.6 show differences between the neutral current and muon-removed charged current events, the distributions for events above the thresholds given in Figure 6.2 are much more similar, suggesting that nearly all the differences have been removed by only looking at muon-removed charged current and neutral current events that represent a background to the selection of charged current events. At this point, it has been established that
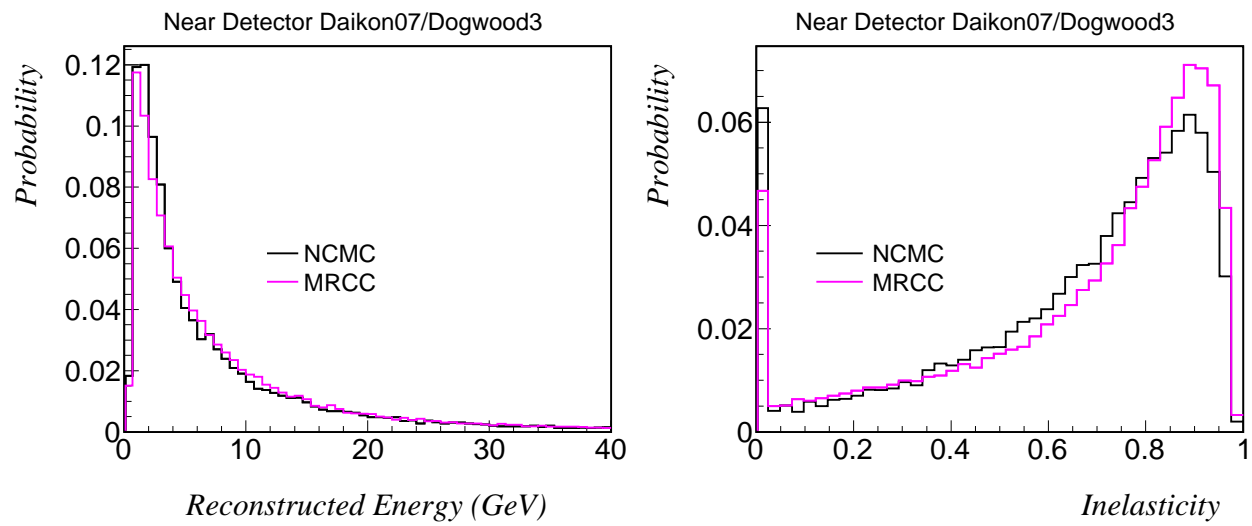

Figure 5.7: Comparison of neutral current Monte Carlo to muon-removed charged current events (MRCC). Note that these plots are area-normalized. Also, muon-removed charged current seems to be higher at high inelasticity. This does not mean that the neutral current Monte Carlo is overestimated at high inelasticity as neutral current Monte Carlo and muon-removed charged current Monte Carlo are intrinsically different (Daikon04/Dogwood7 Monte Carlo and reconstruction).

muon-removed charged current data and Monte Carlo events come from similar distribution charged current events. Furthermore, the muon-removed charged 
current Monte Carlo and neutral current Monte Carlo are also similar in there kinematic distributions such as their reconstructed energy and inelasticity. This is sufficient evidence of the ability of muon-removed charged current events to act as an independent source of hadronic showers.

Not all the muon-removed charged current events mimic the neutral current background. Only muon-removed charged current events which have values of $\mu \mathrm{ID}-\mathrm{A}$ or $\mu \mathrm{ID}-\mathrm{B}$ above the thresholds given in equation (6.2) are events which mimic the neutral current background to the charged current selection. Before this subset of events is discussed in detail, we consider the distribution of muon-removed charged current events for $\mu \mathrm{ID}-\mathrm{A}$ and $\mu \mathrm{ID}-\mathrm{B}$ to ensure there is no significant difference in the fraction of muon-removed charged current events selected in data and Monte Carlo. In Figure $5.8 \mu \mathrm{ID}$ $\mathrm{A}$ and $\mu \mathrm{ID}-\mathrm{B}$ distributions for all muon-removed charged current data and Monte Carlo events are shown. There is excellent agreement overall, with a slight under-estimation by the Monte Carlo of data at high $\mu$ ID-B values, but over-estimation of the neutral current distribution at low $\mu \mathrm{ID}-\mathrm{A}$ and $\mu \mathrm{ID}-\mathrm{B}$ values. Interestingly, the comparison of muon-removed charged current data and Monte Carlo as a function of $\mu \mathrm{ID}-\mathrm{A}$ and $\mu \mathrm{ID}-\mathrm{B}$ is similar to the data and Monte Carlo comparisons of $\mu \mathrm{ID}-\mathrm{A}$ and $\mu \mathrm{ID}-\mathrm{B}$ distributions for the nominal Monte Carlo. That is, in Figure 4.11 and Figure 4.3 we see an excess of Monte Carlo prediction at low PID values, similar to what is shown in Figure 5.8.

\subsection{The Neutral Current Systematic Error}

As previously stated, muon-removed charged current events that pass the charged current selection mimic neutral current events that contribute to the charged current-sample background. Therefore, comparing data and 


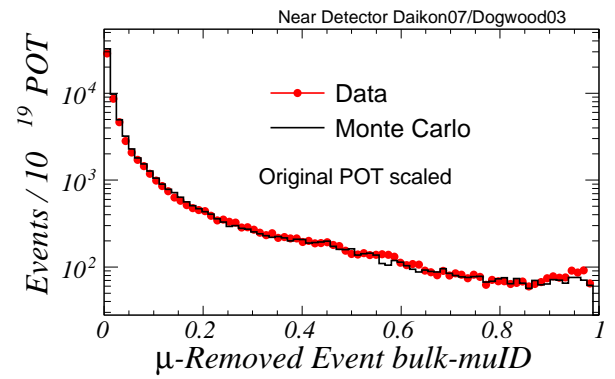

(a) $\mu \mathrm{ID}$-A for $\mu$-removed events

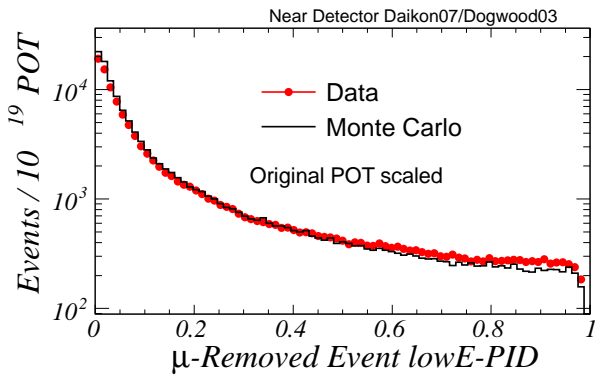

(b) $\mu \mathrm{ID}$-B for $\mu$-removed events

Figure 5.8: The $\mu \mathrm{ID}-\mathrm{A}$ and $\mu \mathrm{ID}-\mathrm{B}$ (see Chapter 4) for muon-removed charged current events. These are charged current showers in which the original muon has been removed from the event and the tracking algorithm re-performed on the remaining hits in the detector. For the events such as those in Figure 5.3, the algorithm reconstructs a second track in the event and we can compute the $\mu \mathrm{ID}$ event separation parameter to define how CC-like the new event is. The Monte Carlo overpredicts the muon-removed charged current event rate at low $\mu \mathrm{ID}$ values and the muon-removed charged current data and Monte Carlo are equal at higher PID values. 
Monte Carlo muon-removed charged current events can be used to study hadronic shower mis-modeling. These mis-modeling studies compare the inelasticity and reconstructed energy distributions for muon-removed charged current data and Monte Carlo events. First the reconstructed energy and inelasticity are considered separately. Figure 5.9 and Figure 5.10 the data to Monte Carlo ratio is fitted with an appropriate approximate function. Then, the muon-removed charged current data to Monte Carlo ratio is simultaneously binned in both energy and inelasticity. This combined study is fitted with a single constant value.

In Figure 5.9, the reconstructed energy distribution of muon-removed charged current data and Monte Carlo events are compared and the ratio of data to Monte Carlo is shown versus versus reconstructed energy of the muon-removed charged current event. This ratio is taken and fitted with the following equation

$$
\frac{M R C C_{\text {data }}}{M R C C_{\text {MonteCarlo }}}=\Delta(x)=1.0+\frac{A}{x+B}+\frac{C}{x^{2}+D}
$$

where $x$ is the energy in $\mathrm{GeV}$. The values and errors on parameters $\mathrm{A}, \mathrm{B}, \mathrm{C}$, and $\mathrm{D}$ for equation (5.2) are shown in the ratio plot of Figure 5.9 (b). This equation gives a rough parametrization of the muon-removed charged current data/Monte Carlo ratio as a function of energy. While the overall ratio of muon-removed charged current data to Monte Carlo is greater than 1, at low energies, where the neutral current background seriously affects the oscillation measurement, the muon-removed charged current data/Monte Carlo ratio is less than 1. This suggests that the neutral current background in this region is smaller than predicted by Monte Carlo. However, parameterizing by neutrino energy is perilous. This is because the desired physics measurement, neutrino oscillations, are also energy dependent. 

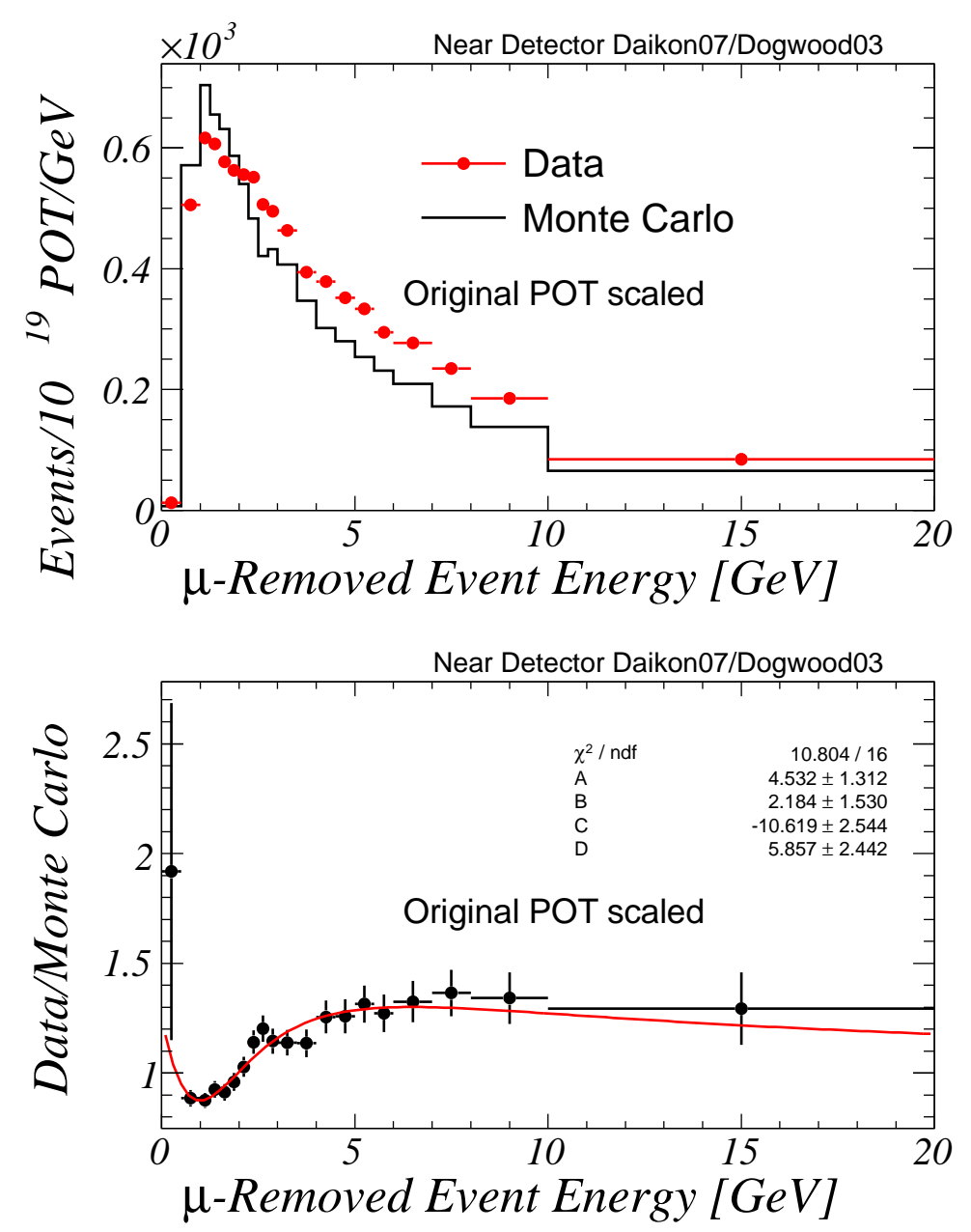

Figure 5.9: Energy spectrum of muon removed Monte Carlo and data after the PID cut. Note the energy dependence in the difference between Monte Carlo and data. Right: ratio of Monte Carlo over data 
The muon-removed charged current data and Monte Carlo inelasticity distributions and ratio are plotted in Figure 5.10. This ratio is parameterized with a fifth order polynomial. The values of the constants and their errors are shown in are shown in Figure 5.10 (b). They are related to the fifth order polynomial by

$$
\frac{M R C C_{\text {data }}}{M R C C_{\text {MonteCarlo }}}=\Delta(\mathbf{y})=A+B \mathbf{y}+C \mathbf{y}^{2}+D \mathbf{y}^{3}+E \mathbf{y}^{4}+F \mathbf{y}^{5}
$$

The fifth order polynomial fit is shown as a red curve in Figure 5.10.

In Section 5.5, this polynomial parameterization is used to re-weight the neutral current Monte Carlo distribution using

$$
N C_{\text {data }}=N C_{\text {MonteCarlo }} \times \Delta(Y)
$$

where $N C_{\text {data }}$ is the neutral current data spectrum and $\Delta(\mathbf{y})$ is the distribution in muon-removed charged current data-Monte Carlo ratio. This is to bring the data into closer agreement with the Monte Carlo and to help ensure that the normalization scaling is independent of the scaling which is developed from the muon-removed charged current data-Monte Carlo ratios. The effect of this correction to the neutral current Monte Carlo distribution is shown in Figure 5.11. As expected, this raises the number of neutral current events at high inelasticity and reconstructed energy and reduces the number of neutral current events at low energy and low inelasticity.

The combination of energy and inelasticity can give one a 2-Dimensional array of data/Monte Carlo ratios. This is shown in Figure 5.12. Figure 5.12 also shows a histogram of the ratios from this two-dimensional array. This histogram has an average of 1.06 with an RMS of roughly 0.15 . The 1.06 value suggests an underprediction of the Monte Carlo over all energies. Recall 

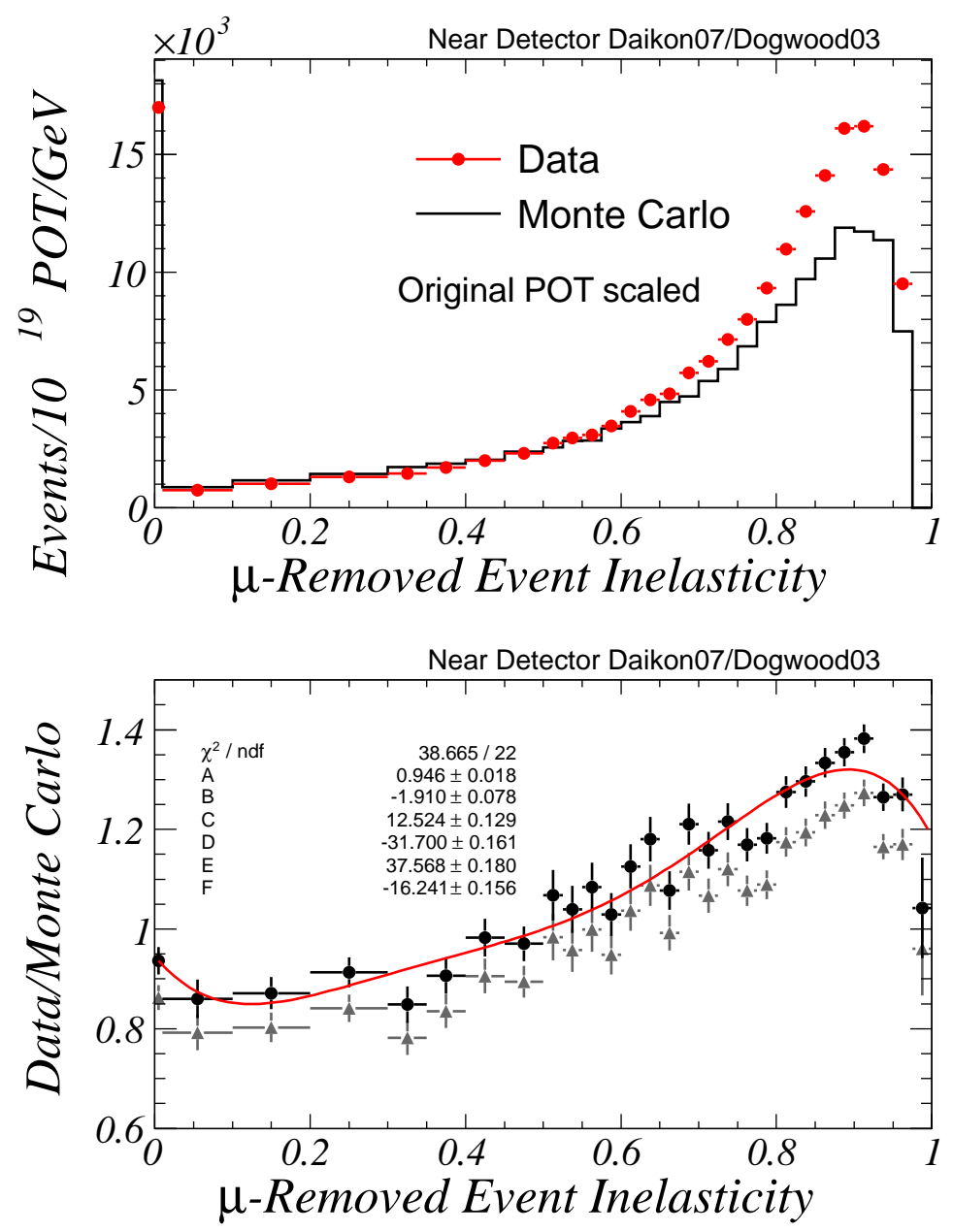

Figure 5.10: The inelasticity distribution of muon removed Monte Carlo and data for events which pass the thresholds given in equation (6.2). Such events mimic the neutral current background to the charged current selection. Note the strong inelasticity dependence in the difference between Monte Carlo and data. Bottom: The ratio of Monte Carlo over data. On the right-hand plot the smooth function is shown as well 


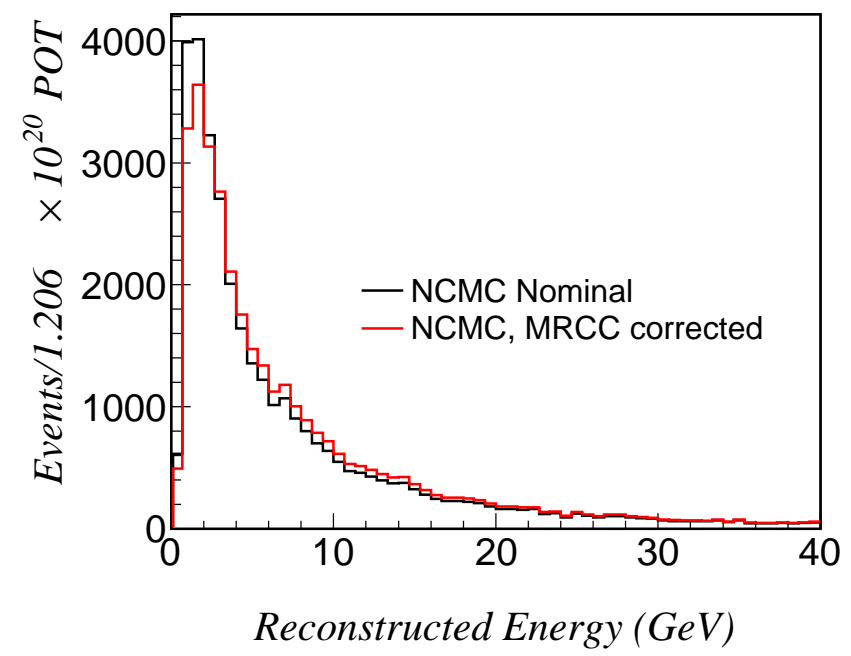

(a) MRCC Energy

Near Detector Daikon07/Dogwood3

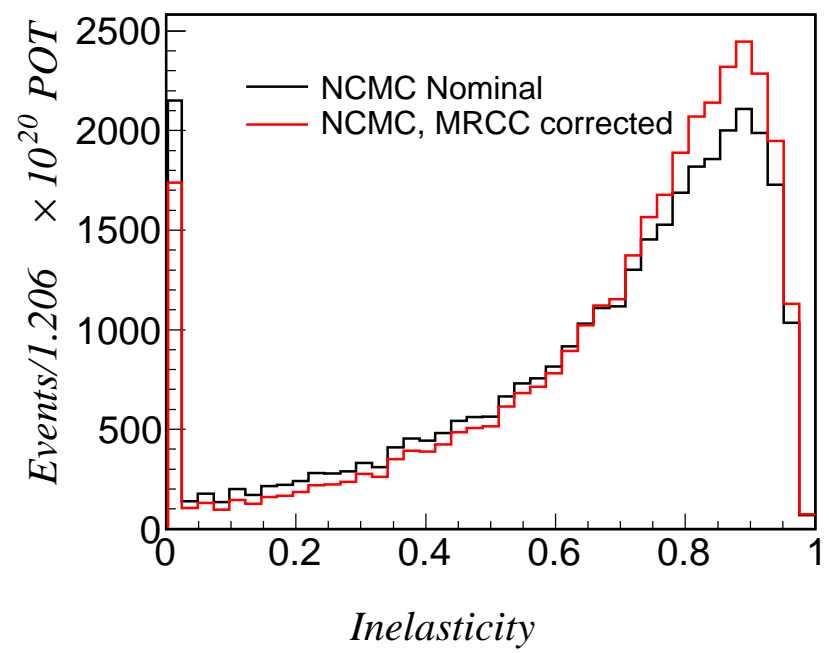

(b) MRCC Inelasticity

Figure 5.11: The energy (top) and inelasticity (bottom) for (black curves) neutral current Monte Carlo events which contaminate the charged current sample (pass the thresholds given in equation (6.2) and (red curves) the same neutral current Monte Carlo contamination corrected by equation (5.3), with values from Figure 5.10, which parameterizes the degree to which MRCC Monte Carlo and MRCC data hadronic showers differ. 
that we are interested in the range of the data to Monte Carlo ratio, not the overall value, which will be better estimated by the normalizatoin study. The 0.15 value for the RMS is the basis for the number we quote as the uncertainty in the hadronic shower modeling of the neutral current background. based on this muon-removed charged current study. A fit of a zeroth order polynomial to Figure 5.12 returns a value of

$$
\Delta=\frac{\text { MRCCData }}{\text { MRCCMonteCarlo }}=1.06 \pm 0.15
$$

The $\chi^{2} / D O F$ value for this fit is $408 / 44$. The central value of 1.06 is not the amount by which we will correct the Monte Carlo, since this averages over all the 2-dimensional energy-inelasticity span in Figure 6.12 and we must extrapolate to the particular energies and inelasticities of relevance for the $\mathrm{NC}$ sample which contaminates the CC sample. The only quantity of relevance is the $15 \%$ RMS. The $15 \%$ fitted uncertainty in this scale factor will be used as the uncertainty in hadronic shower modeling by the Monte Carlo because this fit is the extent to which we can constrain the Monte Carlo hadronic shower modeling using the muon-removed charged current Monte Carlo and data. It is satisfying that this error is comparable to the scaling seen in Figure 5.11.These values indicate that the nominal MINOS Monte Carlo is consistent with our data in terms of predicting the prevalence of charged current-like tracks in hadronic showers, and has the nice feature of using data to constrain the extent to which the Monte Carlo can be off. 


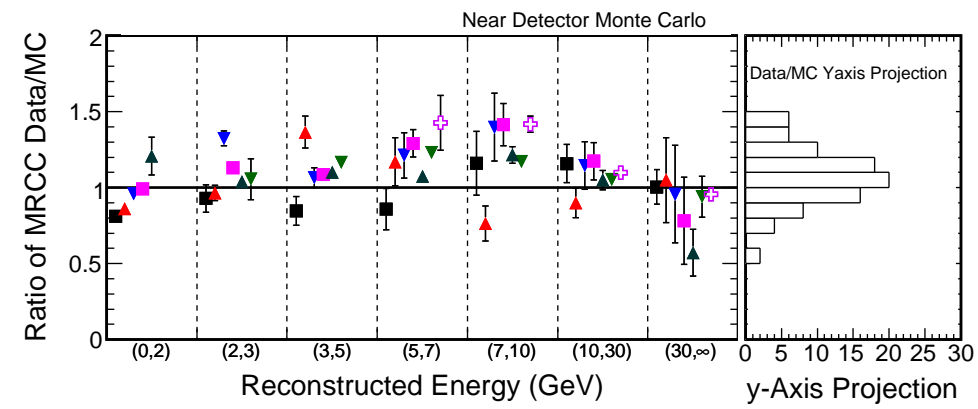

- $0.0 \leq$ inelasticity $<0.3$

$\Delta \quad 0.3 \leq$ inelasticity $<0.5$

$\checkmark \quad 0.5 \leq$ inelasticity $<0.6$

- $\quad 0.6 \leq$ inelasticity $<0.7$

$\Delta \quad 0.7 \leq$ inelasticity $<0.8$

$\checkmark \quad 0.8 \leq$ inelasticity $<0.9$

↔ $0.9 \leq$ inelasticity $<1.0$

Figure 5.12: The ratio of muon-removed charged current data/Monte Carlo for events that pass the event selection. This is shown for different bins of energy and inelasticity. Due to its complicated nature, this was not fitted with a smooth function

\subsubsection{Using the kNN Shower Energy Estimate}

One of the analysis improvements for the current analysis, is the inclusion of a kNN-based shower energy estimator. The new energy estimator uses different distributions of the shower topology in Monte Carlo and a k-Nearest Neighbor algorithm to interpolate the energy of the shower [81]. Much of this will be discussed in later chapters and in the appendix discussing the $\mathrm{k}$ Nearest Neighbor algorithm. The new shower energy estimate is different from the calorimetric energy estimate and produces different reconstructed energy and inelasticity distributions. We expect that this new energy estimate will bring data and Monte Carlo into closer agreement, since the new reconstructed energy is a better estimate of the true energy. Generally, the studies described in this section which do not use the k-NN energy estimate are valid because

- The new kNN energy should not change the number of events selected and does not affect the PID

- The original reconstructed energy is maintained throughout the analysis 

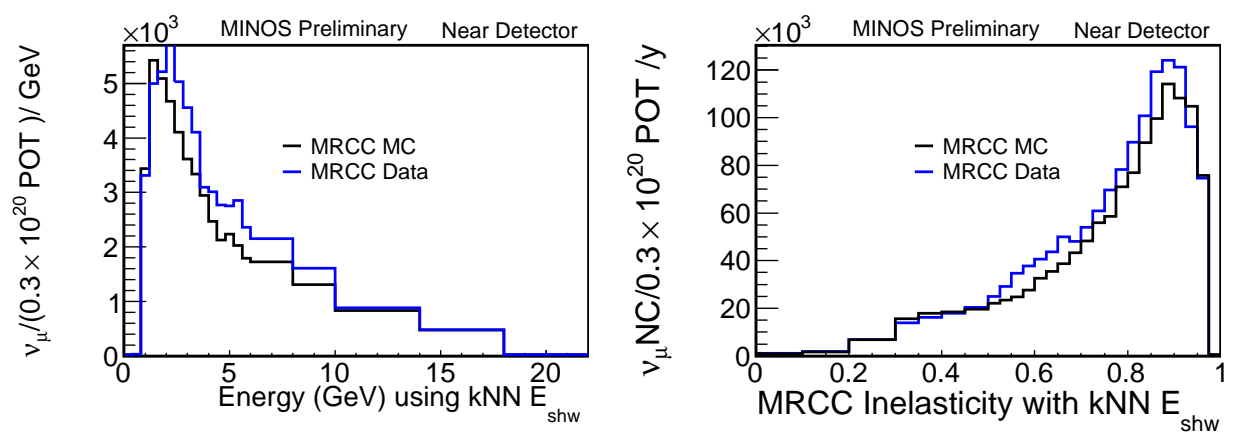

Figure 5.13: The muon-removed charged current data and Monte Carlo reconstructed energy and inelasticity distributions. In these distributions data and Monte Carlo are very similar

and any weighting of the Monte Carlo can still be accomplished through this variable

- The effect of the kNN energy should be the same on Monte Carlo and data.

However, it is necessary that we study the effects of the k-NN energy estimator on the neutral current and muon-removed charged current distributions. In this section, we will consider the effect of the k-NN energy estimate on the neutral current and muon-removed charged current event reconstruction. In Figure 5.13, the k-NN shower energy estimate has been used to create the reconstructed energy and inelasticity. The effect of the k-NN shower estimator is to bring MRCC data and Monte Carlo into closer agreement.

In Figure 5.14 the neutral current and muon-removed charged current area normalized distributions of reconstructed energy and inelasticity are shown for events which pass the thresholds in equation (6.2). In these distributions and in the distributions shown in Figure 5.13, it is obvious that the 
inelasticity in general been raised, with less events having an inelasticity of zero. This is equivalent to stating that the event has no hadronic shower and all of the neutrino energy is transferred to a single final state particle (which is assumed to be a muon although for both the neutral current and muonremoved charged current distributions we know that the final state particle is not a muon). The neutral current and muon-removed charged current distributions are in closer agreement, similar to the muon-removed charged current data and Monte Carlo. In summary, the k-NN energy estimate has the effect of smoothing the distributions into closer agreement.

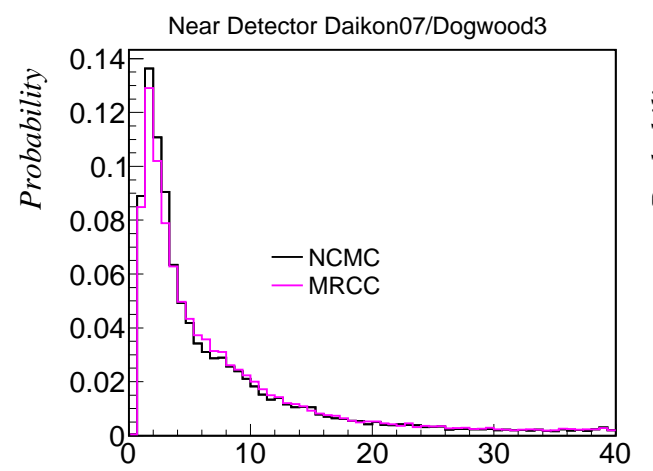

Reconstructed Energy with kNN [GeV]

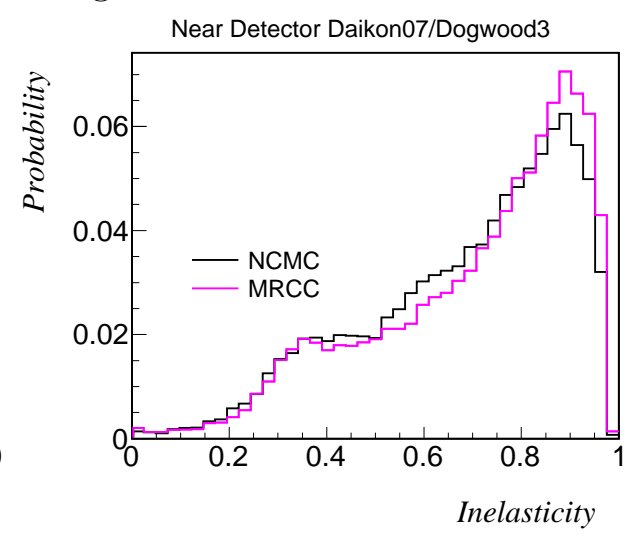

Figure 5.14: The neutral current and muon-removed charged current Monte Carlo reconstructed energy and inelasticity distribution. In these distributions neutral current and muon-removed charged current are very similar.

In Figure 5.15, the neutral current Monte Carlo is shown before and after re-weighted by the inelasticity weight given before. This inelasticity weighting equation was computed without the k-NN energy estimate but the quantities plotted here are with the k-NN energy estimate. Each event is weighted by its pre-k-NN estimate inelasticity, but shown for the inelasticity and reconstructed energy after using the k-NN energy estimate. The trend 
of these weightings on the neutral current distribution is the same as without the kNN shower energy. High inelasticities, high energy events are weighted heavier than those at low inelasticity and low energy. This is expected, as the k-NN energy estimate only improves the accuracy of our energy estimate. We do not expect the values between the k-NN estimated energy and the calorimetric estimated energy to be significantly different.
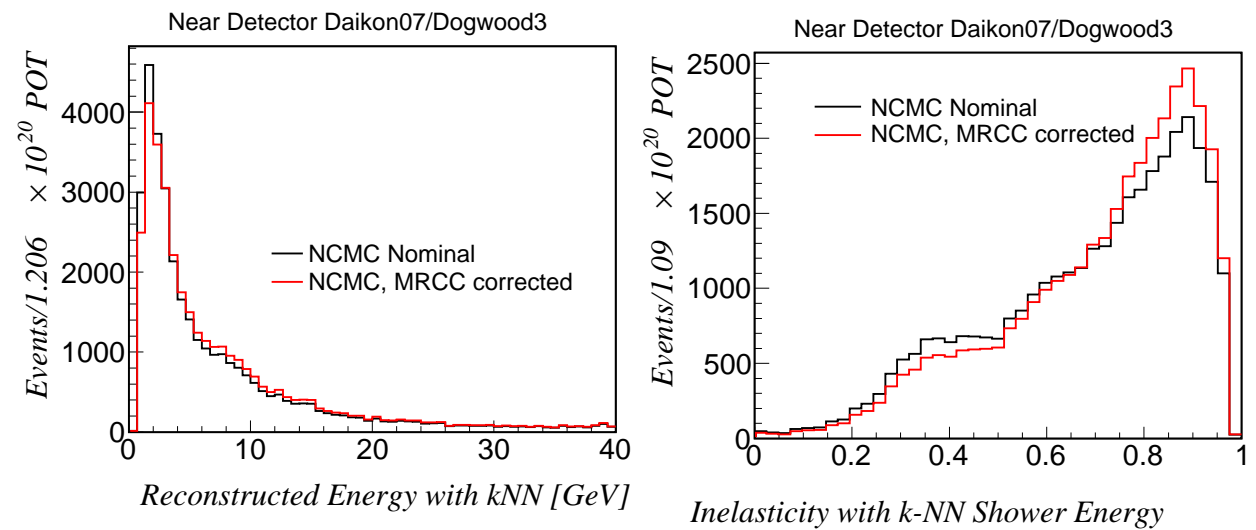

Figure 5.15: This shows the nominal neutral current background (black) and the neutral current background weighted by the function equation (5.3) (red) with kNN shower energy.

In summary, the k-NN energy estimate does impact the muon-removed charged current and neutral current energy and inelasticity distributions. However its effect is mostly to move low inelasticity and low energy events to higher inelasticities and energies. Because the muon-removed charged current Monte Carlo overestimates data at low inelasticity and energy and underestimates data at high energy and high inelsticity, this has the effect of bringing data and Monte Carlo into closer agreement. Therefore, our maximum error is given by the distributions using only the calorimetric energy estimates. 


\subsection{The Neutral Current Background Normalization Er- ror}

The muon-removed charged current data-Monte Carlo comparison studies discussed in the previous section provide a constraint on the size of the Monte Carlo shower mis-modeling contribution to the neutral current systematic error. However, nothing is learned about the neutral current normalization, as both the muon-removed charged current event are completely independent of neutral current cross sections. The total neutral current systematic uncertainty is a combination of shower modeling uncertainties, which can lead to distortions in the neutral current Monte Carlo distribution shapes, and a cross section and track reconstruction uncertainty, which will lead to overall increases and decreases of neutral current background in the charged current event sample.

To estimate the normalization contribution to the neutral current systematic error, we use the PID distribution for charged current candidates in the Near Detector for both data and Monte Carlo. The two components of the Monte Carlo (charged current, and neutral current) are scaled separately until a best-fit is reached between the Near Detector data and Monte Carlo. This scale fitting is done by assuming that all the discrepancy between data and Monte Carlo is due to neutral current normalization (an unlikely claim) and again by scaling both distributions and finding the absolute best-fit of data scaling both charged current and neutral current events. This is a produces an

estimate for a maximum normalization uncertainty. The process of this study is as follows:

- Begin with three histograms - data, charged current Monte Carlo and 
neutral current Monte Carlo that has been re-weighted by one of the smooth functions from the muon-removed charged current analysis.

- Using one of the $\mu \mathrm{ID}-\mathrm{B}$ perform the following fits:

- The data histogram is maintained while the neutral current and charged current Monte Carlo histograms are scaled to minimize the log-likelihood that the data is composed of the neutral current Monte Carlo and charged current Monte Carlo.

- The ROOT function TFractionFitter returns the percent of charged current and neutral current that best fits the data, and an error for this number

This study was done twice, once with a fixed charged current Monte Carlo and once where the normalization of the charged current Monte Carlo can vary as well. The results for both of these fits along with the unfitted charged current and neutral current values are shown in Table 5.5. The $\mu \mathrm{ID}-\mathrm{B}$ was used as the fitting distribution because it is relatively independent of the inelasticity and energy distributions. Therefore, we can hope that this error estimate will not be directly affected by the effects seen in the previous sections. In the first row of Table $\mathrm{C}$ the relative percentage of charged current and neutral current events in the nominal Monte Carlo is shown. The second row of Table $\mathrm{C}$ shows the fitted value of the charged current and neutral current populations after just the neutral current distribution normalization is adjusted to improve the fit to the Near Detector data. The third row shows the relative charged current and neutral current populations after both the charged current and neutral current components are floated in a fit to the ND data. The charged 


\begin{tabular}{|l|l|l|l|l|l|}
\cline { 2 - 5 } \multicolumn{1}{c|}{} & \multicolumn{2}{l|}{$\begin{array}{l}\text { Percentage of the Total } \\
\text { Distribution }\end{array}$} & \multicolumn{2}{l|}{$\begin{array}{l}\text { Original } \\
\text { scaled by }\end{array}$} & \multicolumn{2}{|c|}{ distribution } \\
\cline { 2 - 5 } & $\begin{array}{l}\text { charged } \\
\text { current }\end{array}$ & $\begin{array}{l}\text { neutral cur- } \\
\text { rent }\end{array}$ & $\begin{array}{l}\text { charged } \\
\text { current }\end{array}$ & $\begin{array}{l}\text { neutral cur- } \\
\text { rent }\end{array}$ & $\chi^{2}$ \\
\hline Before & $96.6 \%$ & $4.06 \%$ & 1.0 & 1.0 & NA \\
\hline Fixed & $96.6 \%$ & $3.8 \pm 0.13 \%$ & 1.0 & $.935 \pm .032$ & 5.39 \\
\hline Unfixed & $96.0 \pm 0.3 \%$ & $3.94 \pm 0.14 \%$ & $.994 \pm 0.004$ & $0.97 \pm 0.003$ & 5.33 \\
\hline
\end{tabular}

Table 5.3: Normalization values for neutral current and charged current Monte Carlo before and after re-weighting. The columns represent (1) the name of the fit,the percentage of (2) charged current and (3) neutral current in the total Monte Carlo, the value by which the nominal (4) charged current and (5) neutral current Monte Carlo distributions are scaled, and (6) the $\chi^{2}$ value of the fit. The rows represent (A) the "Unfitted" original values of the charged current and neutral current Monte Carlo. In this row we see the nominal amount of charged current, and neutral current Monte Carlo. Because there is no fit, the $\chi^{2}$ is not found. (B) the "charged current Fixed" fixed of the charged current and neutral current Monte Carlo. In this row, the charged current distribution is held fixed and only the neutral current Monte Carlo varies. (note that the scale factor for the charged current distribution is 1.0) and $(\mathrm{C})$ the "charged current Unfixed" fit of the charged current and neutral current Monte Carlo. Here both the charged current and neutral current Monte Carlo distributions are allowed to vary (with a range in the charged current direction of $\pm 1.6 \%$ ). the charged current normalization error). Note that the $\chi^{2}$ values of the fits are reasonable. 
current component is constrained by our charged current normalization error of $1.6 \%$.

In Figure 5.16 we see the Near Detector data, charged current Monte Carlo and muon-removed charged current scale ed neutral current Monte Carlo before and after scaling for data-Monte Carlo best fit. This corresponds to the fit of the third row in table Table 5.5. As can be seem the PID distribution in Monte Carlo better describes the data after the fit.

Figure 5.17 shows result from a similar normalization fitting except with leaving the charged current distribution stationary and only fitting the neutral current distribution.

The studies shown in this chapter were performed with an updated Monte Carlo and reconstruction algorithms. However, the original reconstruction and Monte Carlo versions used in these studies were slightly older. The older version gave a slightly larger value for the systematic error. In order to err on the side of caution, the final systematic used the older result, rather than the result using the updated Monte Carlo and reconstructions. Although the answer is slightly different, the methodology used in both cases is exactly the same. Therefore, a description of the study is not required. The study with the original Monte Carlo and reconstruction is discussed in Appendix C.

\subsection{Summary}

The combined uncertainty is the addition of the uncertainty in maximum normalization and the muon-removed charged current study efficiency error in quadrature. Using the spread of the muon-removed charged current data to Monte Carlo as the estimate of the neutral current shower modeling 


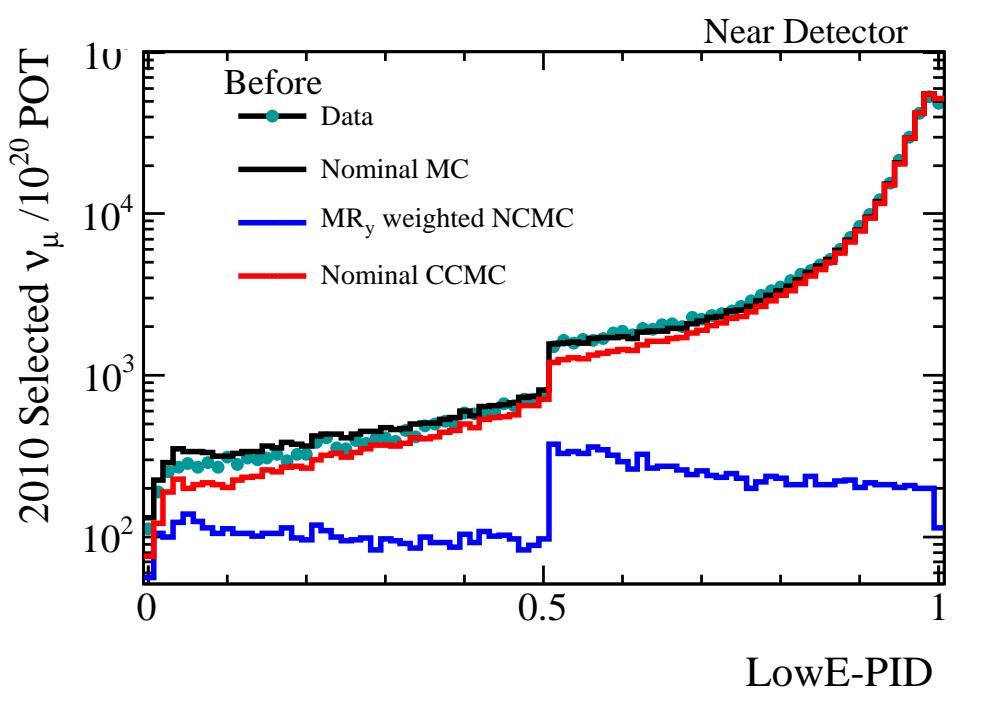

(a) Before

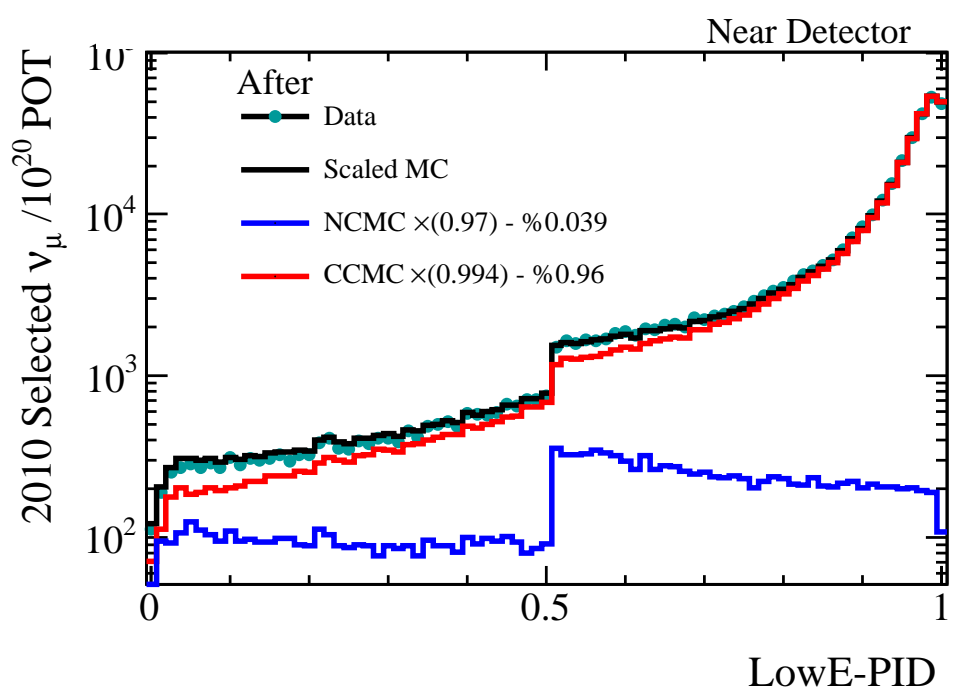

(b) After

Figure 5.16: As a function of $\mu \mathrm{ID}-\mathrm{B}$ using the thresholds in equation (6.2). the charged current Monte Carlo, neutral current Monte Carlo weighted by the smooth energy scaling and near detector data before (left) and after(right) varying the neutral current Monte Carlo normalization while keeping the charged current Normalization fixed. 144 


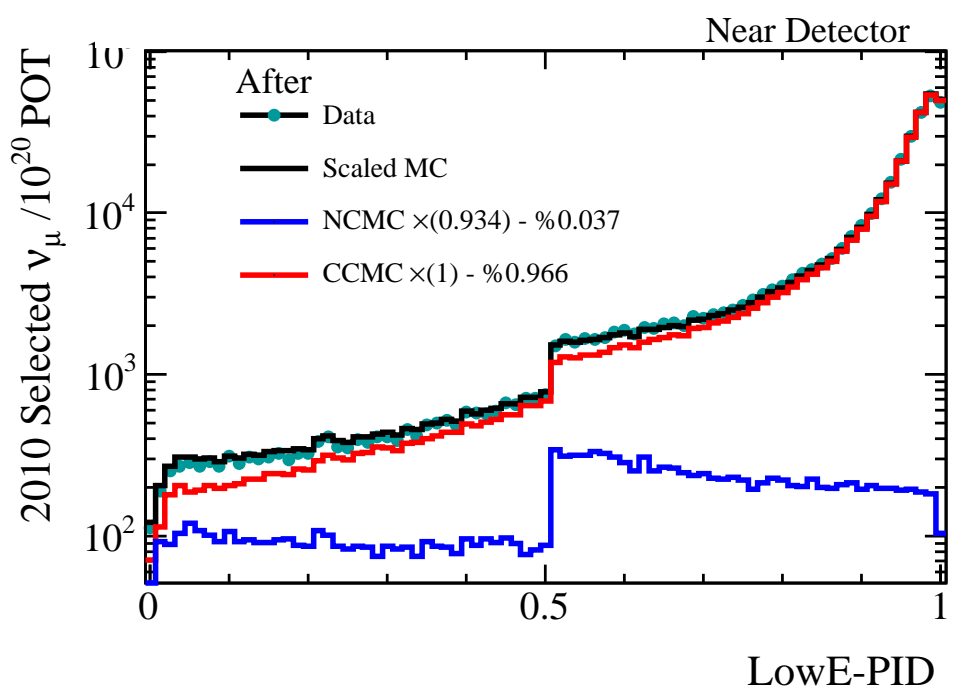

Figure 5.17: As a function of $\mu$ ID-B using the thresholds in equation (6.2). the charged current Monte Carlo, neutral current Monte Carlo weighted by the smooth energy scaling and near detector data before (left) and after(right) varying the neutral current Monte Carlo normalization while keeping the charged current Normalization fixed 
uncertainty and the maximum difference in the neutral current background from the normalization study gives a total error of

$$
\sigma_{\text {neutralcurrent }}=\sqrt{(15 \%)^{2}+(7 \%)^{2}}=17 \%
$$

A $20 \%$ error is considered a safe estimate of our neutral current systematic uncertainty. This value comfortably covers the errors due to shower modeling, track reconstruction, and the neutral current cross section. ${ }^{3}$

\footnotetext{
${ }^{3}$ In the published analysis, the normalization uncertainty was quoted to be a larger number due to some last minute discrepancies discovered when reconstruction algorithms were changed. However, the $20 \%$ overall systematic uncertainty was sufficient to cover both results. These effects are discussed in Appendix C.
} 


\section{Chapter 6}

\section{Optimization of the Charged Current Selection Threshold}

In Chapter 4, we presented a method for extending the selection of charged current events to very low neutrino energies. This extension utilized a similar k-NN based alogirthm used in the previous MINOS analysis [69], but removed some of the biases that lead to decreased efficiency at low energy. In order to increase the charged current sample without increasing the neutral current background, a new low-energy k-NN $\mu \mathrm{ID}$ paramter, $\mu \mathrm{ID}-\mathrm{B}$, was introduced. $\mu \mathrm{ID}-\mathrm{B}$ is not as selective as $\mu \mathrm{ID}-\mathrm{A}$, so it must be utilized with care or it will allow more neutral current events to contaminate the charged current selection. In this chapter, we discuss an optimized selection which properly balances the need to aquire more events at low energy and the desire to keep the neutral current background of the charged current sample at a minimum.

Recall that the purpose of increasing the number of low-energy neutrino events is not for the precision measurement of the oscillation quantities, but to have a better distribution of events around the oscillation minimum. Other theories of neutrino disappearance, such as quantum decoherence or neutrino decay $[32,82]$ predict a very different functional form from that shown in Figure 1.1. Thus, the ability to acquire more events with $E<2 \mathrm{GeV}$ allows the MINOS experiment to provide a convincing demonstration that these other models are not viable descriptions of neutrino disappearance. 
$\mu \mathrm{ID}-\mathrm{B}$ was designed to act as a pathway for otherwise excluded events to be included into the charged current event sample. There are two obvious methods of incorporating $\mu \mathrm{ID}-\mathrm{B}$. The first is to select events which pass both $\mu \mathrm{ID}-\mathrm{A}$ and $\mu \mathrm{ID}-\mathrm{B}$. The second method is to pass events which pass either $\mu \mathrm{ID}-\mathrm{A}$ and $\mu \mathrm{ID}-\mathrm{B}$. However, the first method will not work because the events below 10 planes would be lost, as they are all rejected by $\mu \mathrm{ID}-\mathrm{A}$, and $\mu \mathrm{ID}-\mathrm{B}$ may reject events accepted by $\mu \mathrm{ID}$-A. Therefore, the second method of using a logical $(V)$ is used.

The question of how to accept events using these two PIDs is reduced to where the thresholds should be placed on these two charged current selection parameters. Events with a value above a selection threshold are accepted as part of the charged current event sample. Previously $\Theta_{A}$ was set at [33]

$$
\Theta_{A}=0.3
$$

However, $\mu \mathrm{ID}-\mathrm{B}$ had not been developed at this point (the threshold is effectively set at one, $\left.\Theta_{A}=1.0\right)$. Because we have introduced $\mu \mathrm{ID}-\mathrm{B}$, we must reevaluate the thresholds, $\Theta_{A}$ and $\Theta_{B}$, such that we can discover a combination of values which increase our selection at low enrgy and maintaining a low neutral current background. There are many factors that need to be considered when optimizing the selection of charged current events. These include:

1. the ability to discriminate between non-oscillation models like decay and decoherence and oscillations,

2. the statistical errors on the oscillation measurement, and

3. the neutral current systematic error. 
Through analysis of these three factors, the final thresholds for $\mu \mathrm{ID}-\mathrm{A}, \Theta_{A}$, and $\mu \mathrm{ID}-\mathrm{B}, \Theta_{B}$, were decided as

$$
\Theta_{A}=0.25, \text { and } \Theta_{B}=0.5
$$

Studies to optimize the threshold values are described in the following sections. In brief, these thresholds optimizes the discrimination against alternate neutrino disapperance models [32, 82] while maintaining sensitivity for oscillations. In Section 6.1 the sensitivity to oscillations over decay and decoherence will be discussed. In Section 6.2, we study the effect on contours of the oscillation analysis, and in Section 6.3, we study the effect of increasing the neutral current background on the systematic error. These studies were performed with the analysis improvements which will be discussed in Section 7.2. Wrong-sign events were also included.

\subsection{Model Sensitivity}

In order to measure the sensitivity of model discrimination on the charged current selection, we simulated a 'fake data' sample of events in the MINOS Far Detector. This is created by applying an oscillation model to Monte Carlo events. The oscillation parameters used for producing the fake data sample were

$$
\Delta m_{32}^{2}=2.38 \times 10^{-3} e V^{2}, \text { and } \sin ^{2}\left(2 \theta_{32}\right)=1.0
$$

Because the fake data is oscillated, we expect it to find that the fake data is consistent with neutrino oscillations and inconsistent with models of neutrino decay or neutrino decoherence[32, 82]. Therefore, we would expect the log-likelihood for a fake data sample to be consistent with either decay or 
decoherence models to be larger than the log-likelihood for the fake data to be consistent with the oscillation model. We use the difference between the log-likelihoods (of decay or decoherence versus oscillation models) as a metric of the inconsistency of these models. This metric was evaluated as a function of the thresholds for the charged current selection. Ideally, the best thresholds would maximize this difference between oscillation and decay or decoherence models.

Figure 6.1 shows the result of a fake data study in which the oscillated FD fake data was fit to a generic decay model and Figure 6.2 is the similar graph comparing the oscillation hypohtesis to a generic decoherence model. In both cases, the likelihood was computed for the decay or decoherence hypothesis and for the best oscillation fit. The difference between these two log likelihoods is plotted on a color axis versus the threshold values for the two PIDs. The $\mathrm{Y}$-axis represents the threshold of $\mu \mathrm{ID}-\mathrm{B}, \Theta_{B}$ and the $\mathrm{X}$-axis represents the threshold value of $\mu \mathrm{ID}-\mathrm{A}, \Theta_{A}$. Therefore, values up and to the right are higher thresholds and stricter requirements for the charged current selection and values down a to the left are lower threshold values and a more relaxed charged current selection.

Figure 6.3 shows the oscillation hypothesis log-likelihood by itself. Note that the scale on this plot is significantly different than the color scales on Figure 6.1 and Figure 6.2. The oscillation model hypothesis is very weakly associated with the thresholds for the two PIDs and its shape the oscillation likelihood is much smaller than decay or decoherence likelihoods and its shape seems to be determined by statistical fluctuations and therefore not reliable.

Two points are highlighted on each of the plots. The star represents $\mu$ ID-A threshold at 0.3 used for the MINOS $\nu_{\mu}$ disappearance analysis in 2008. 


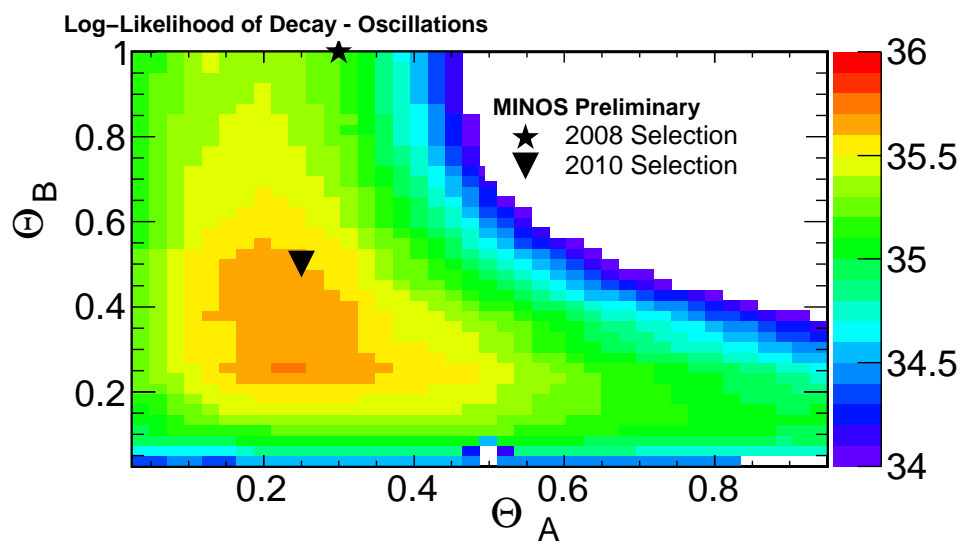

Figure 6.1: Difference in the log-likelihood of the decay and oscillation hypotheses for various different values of the thresholds for $\mu \mathrm{ID}-\mathrm{A}$ (X-axis) and $\mu \mathrm{ID}-\mathrm{B}$ (Yaxis). Larger differences between the log-likelihoods represent a stronger disfavorment of the neutrino decay hypothesis.

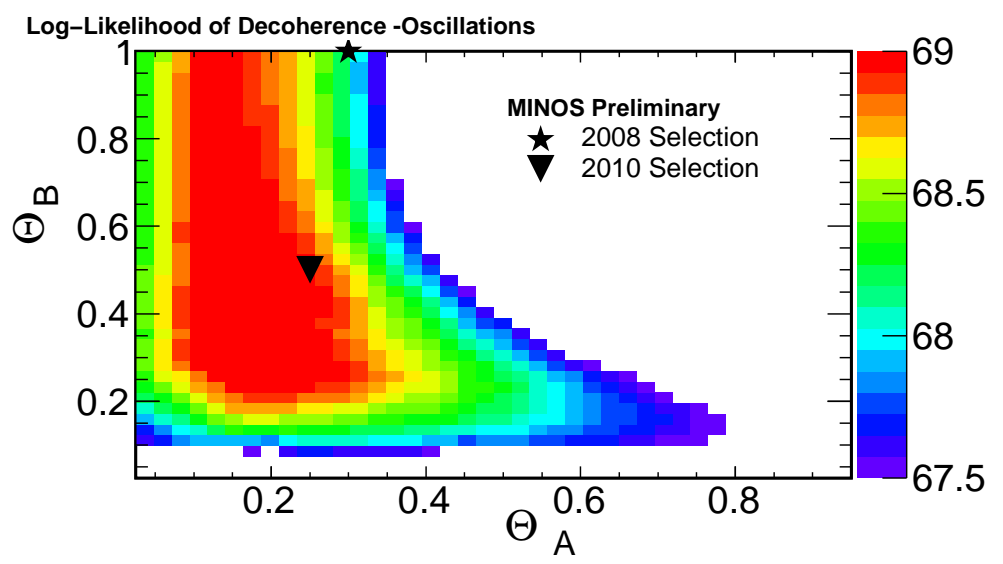

Figure 6.2: Difference in log-likelihood for decoherence and oscillation hypotheses for various different values of the thresholds for $\mu \mathrm{ID}-\mathrm{A}$ (X-axis) and $\mu \mathrm{ID}-\mathrm{B}$ (Yaxis). Larger differences between the log-likelihoods represent a stronger disfavorment of the neutrino decoherence hypothesis. 


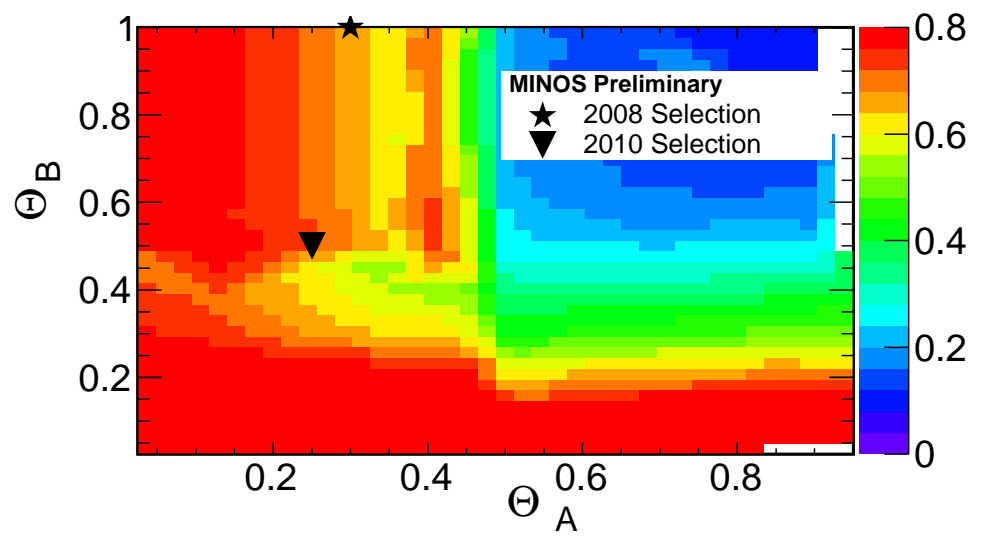

Figure 6.3: Log-likelihood for oscillation hypothesis on fake data. Here, the changes in the log-likelihood are smaller than they are for the decay and decoherence hypotheses.

Its placement at the top of the "Alternate $\mathrm{kNN"} \mathrm{(or} \mu \mathrm{ID}-\mathrm{B}$ ) represents the fact that $\mu \mathrm{ID}-\mathrm{B}$ did not exist in 2008. Therefore, the $2008 \nu_{\mu}$ disappearance analysis effectively had a threshold at 1.0, where no events accepted by $\mu$ ID-B were accepted. The triangle represents the final optimized threshold as stated in equation (6.2). We see that this value is near a point of optimization, where the difference between the log-likelihood of the decay and decoherence hypotheses and the oscillation hypothesis is maximized. However, it is right and upwards of this maxima, due to the need to reduce systematic errors due to neutral current backgrounds.

\subsection{Oscillation Sensitivity}

In addition to discriminating between decay, decoherence and oscillation hypotheses, the selection of charged current events must also optimize the MINOS measurement of the oscillation parameters, $\Delta m_{32}^{2}$ and $\sin ^{2}\left(2 \theta_{32}\right)$. 
In this section we present a series of studies to understand the effect of the charged current selection on the oscillation parameters. In the first study, the allowed region of $\Delta m_{32}^{2}$ and $\sin ^{2}\left(2 \theta_{32}\right)$ values were measured at different values of the thresholds $\Theta_{A}$ and $\Theta_{B}$. We seek to set the selection values such that they would not increase the allowed region of values for $\Delta m_{32}^{2}$ and $\sin ^{2}\left(2 \theta_{32}\right)$ beyond the area set by using simply the $\mu \mathrm{ID}-\mathrm{A}$ selection set in equation (6.1).

In Figure 6.4(a), the contours for a PID cut at $\Theta_{A}=0.025$ and $\Theta_{B}=$ 0.025 in red and the contours for threshold given by equation (6.1) in black. Because these thresholds are very near zero, the red curve represents how a relaxed charged current selection can adversely affect the contours. The values of the red curve allow enough neutral current events into the event selection that the overall shape of the oscillation contours primarily in the direction of $\sin ^{2}\left(2 \theta_{32}\right)$. Note, however, that the difference in $\Delta m_{32}^{2}$ is very small, especially when $\sin ^{2}\left(2 \theta_{32}\right)=1$.0.In Figure 6.4(b) the contours for the values in Figure 6.2 (and used in the current analysis) are compared to the selection for the 2008 MINOS $\nu_{\mu}$ disappearance analysis. Note that the contours are basically the same for the two selections. In fact, above some minimum threshold (around 0.2 for both $\mu \mathrm{ID}-\mathrm{A}$ and $\mu \mathrm{ID}-\mathrm{B})$, the $\nu_{\mu}$ disappearance analysis is not greatly affected by the charged current selection.

Because the differences in the oscillation contours are very small, it was necessary to consider the effect changing the thresholds would have on the oscillation measurement in a different way. We now consider the effect of the charged-current selection on the likelihood of oscillations to describe the behavior of the oscillated vake data. This is different from comparing decay and decoherence from oscillations because we are not considering other possible hypotheses for neutrino disappearance. 


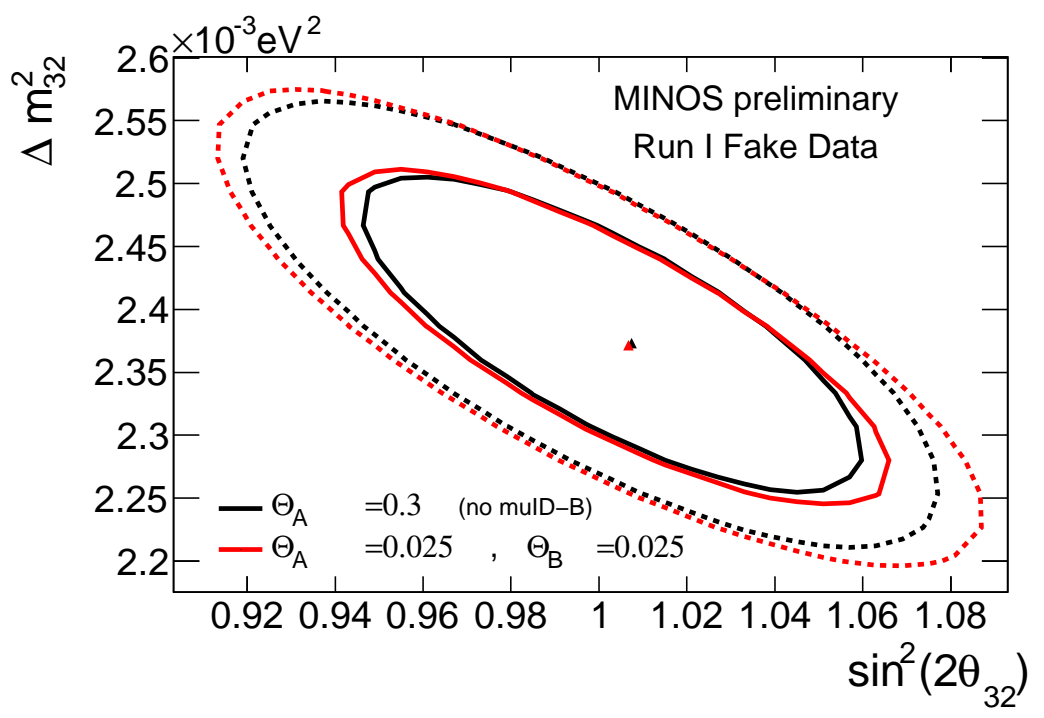

(a)

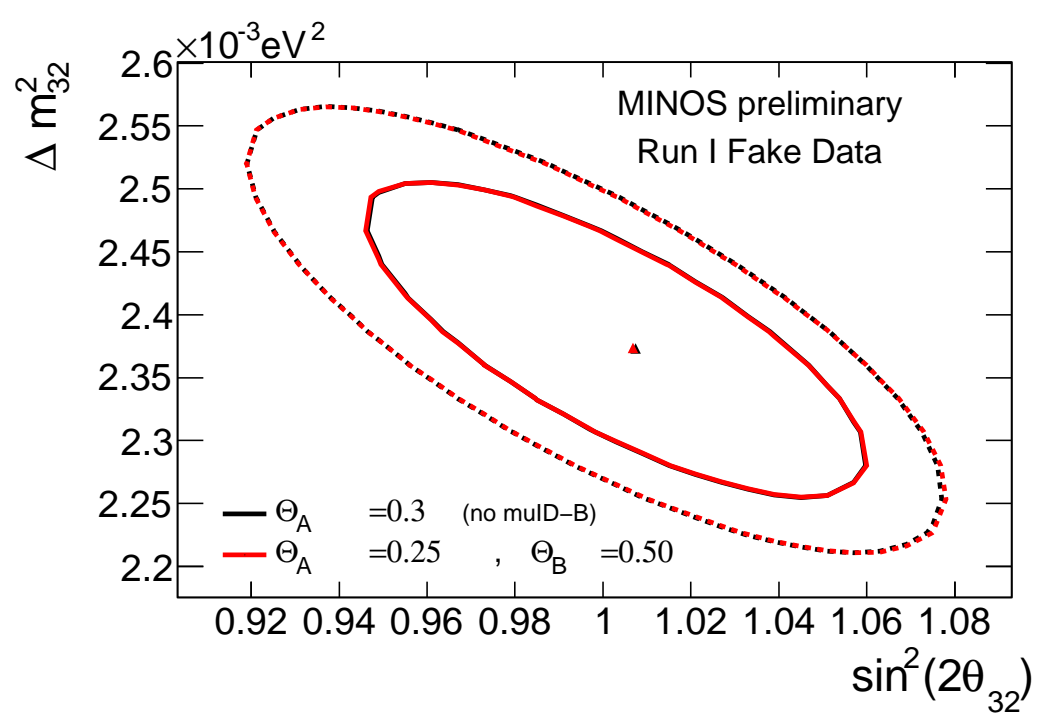

(b)

Figure 6.4: The oscillation contours for the threshold on $\mu \mathrm{ID}-\mathrm{A}$ used in the 2008 MINOS $\nu_{\mu}$ disappearance analysis is shown in black. This is compared to a contour in red, which represents (a) Thresholds on $\mu$ ID-A and $\mu$ ID-B which allow nearly all track-events in, (b) The contours for the threshold values given in Figure 6.2, which were used in the current MINOS $\nu_{\mu}$ disappearance analysis. The solid curves represent the contours of $68 \%$ confidence, whereas the dashed curves represent the contours of $99 \%$ confidence. 
For the fake data oscillated to the parameters given in equation (6.3), five different points in $\Delta m_{32}^{2}$ and $\sin ^{2}\left(2 \theta_{32}\right)$ were selected. These five points were:

- (A) $\Delta m_{32}^{2}=0.0232$ and $\sin ^{2}\left(2 \theta_{32}\right)=1.0$

- (B) $\Delta m_{32}^{2}=0.0247$ and $\sin ^{2}\left(2 \theta_{32}\right)=1.0$

- (C) $\Delta m_{32}^{2}=0.0250$ and $\sin ^{2}\left(2 \theta_{32}\right)=0.95$

- (D) $\Delta m_{32}^{2}=0.0256$ and $\sin ^{2}\left(2 \theta_{32}\right)=1.0$

- (E) $\Delta m_{32}^{2}=0.0265$ and $\sin ^{2}\left(2 \theta_{32}\right)=0.95$.

For each of these points, the log-likelihood of the oscillation hypothesis was monitored as the selection thresholds were varied. This is plotted in Figure 6.5, where the subfigure letters match with the letters and values given above. The goal is to maximize the absolute likelihood for neutrino oscillations. The selection thresholds, $\Theta_{A}$ and $\Theta_{B}$ vary along the $\mathrm{X}$ and $\mathrm{Y}$ axis respectively. For each combination, the oscillation likelihood is shown as a color. (A), which represents a value less than the best fit and at maximal mixing, prefers selection thresholds near the values given in equation (6.2). (B) and (D), which are at maximal mixing but above the best-fit value, display preference towards no PID cut. (C) and (E), which are at below maximal mixing, display a maximum likelihood near the values given in equation (6.2).

Figure 6.5(f) is the multiplication of (B) and (C). Each color value represents the likelihoods at the same point in (B) and (C) multiplied. Because both points (B) and (C) roughly lie on the $68 \%$ confidence level contour, this plot demonstrates how the overall likelihood along the $68 \%$ contour changes. 

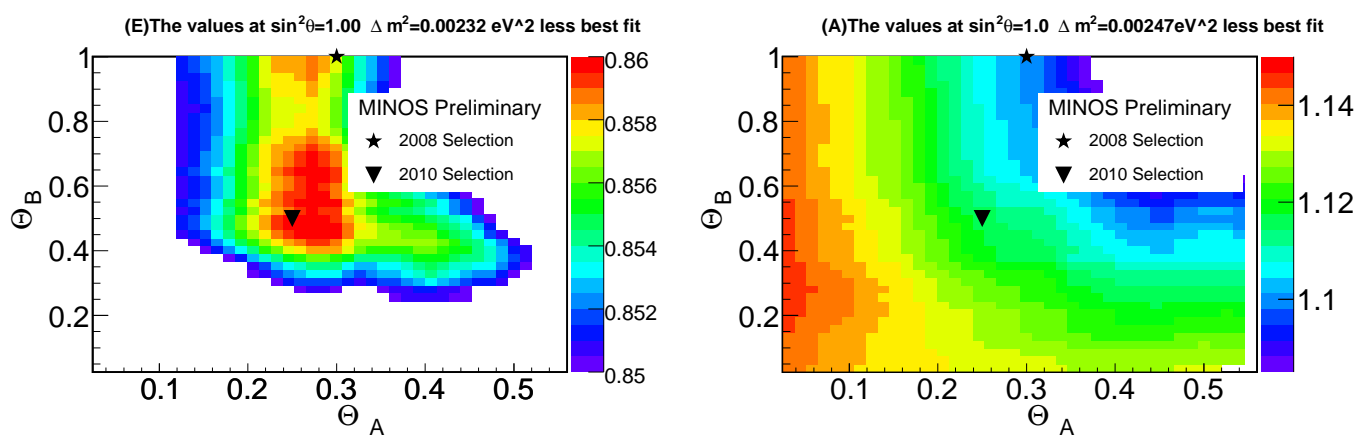

(a) $\Delta m_{32}^{2}=0.0232$ and $\sin ^{2}\left(2 \theta_{32}\right)=1.0$

(b) $\Delta m_{32}^{2}=0.0247$ and $\sin ^{2}\left(2 \theta_{32}\right)=1.0$
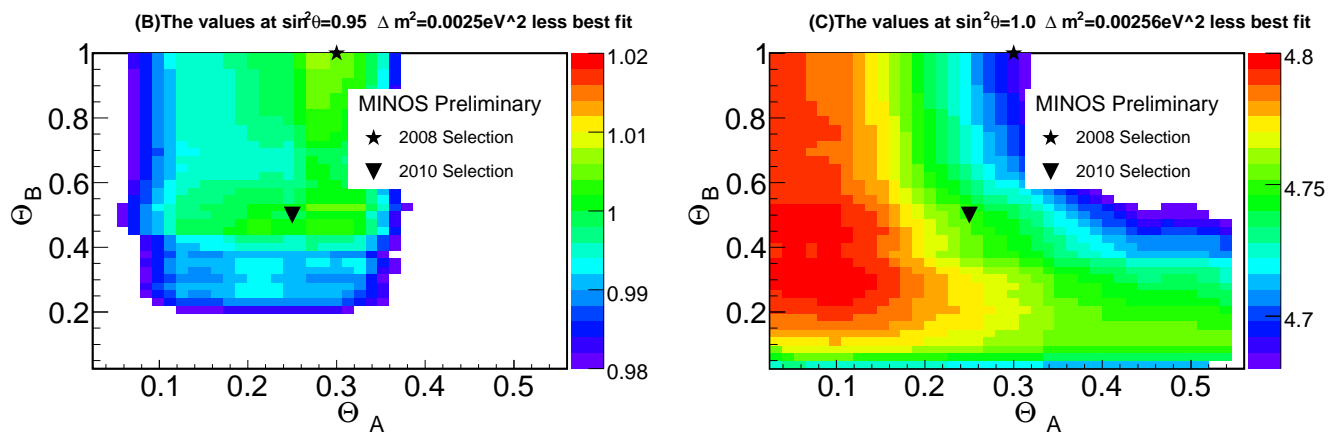

(c) $\Delta m_{32}^{2}=0.0250$ and $\sin ^{2}\left(2 \theta_{32}\right)=0.95$

(d) $\Delta m_{32}^{2}=0.0256$ and $\sin ^{2}\left(2 \theta_{32}\right)=1.0$
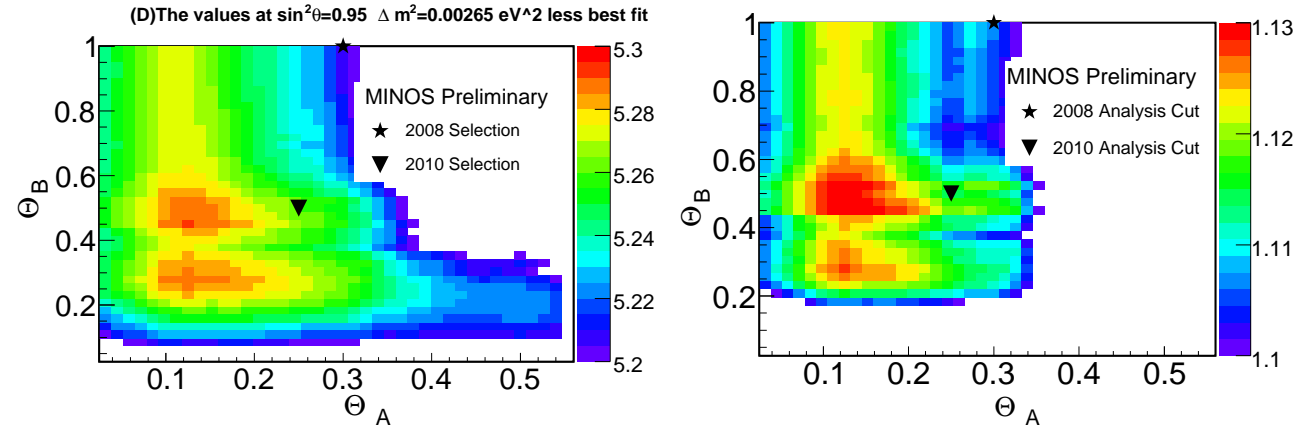

(e) $\Delta m_{32}^{2}=0.0265$ and $\sin ^{2}\left(2 \theta_{32}\right)=0.95$

(f) $B \times C$

Figure 6.5: The likelihoods at five oscillation parameters versus the selection thresholds. (A), (B), and (D) are at maximal mixing. (C) and (E) have $\sin ^{2}\left(2 \theta_{32}\right)=0.95$. (A) through (E) have successively larger values of $\Delta m_{32}^{2}$. Both (A) and (C) display larger likelihoods in the region of the 2010 PID cut. (B) and (D) suggest that something is systematically happening - perhaps shifting the best-fit point further away. (B), (D) and (E) suggest that at non-maximal mixing, the contour finds a minimum near the 2010 PID cut. 
This demonstrates a maximum near the values given in equation (6.2). Overall, we see that the likelihood of oscillations has a maximum near, but not exactly at, the threshold values given in equation (6.2). ${ }^{1}$

\subsection{Neutral Current Contamination}

$\mu \mathrm{ID}-\mathrm{B}$ provides a mechanism for increased efficiency and discrimination between charged current and neutral current events at low energy. This can be seen in Figure 6.6 for events with reconstructed energy less than $2 \mathrm{GeV}$ and 'muon' track lengths less than 20 planes. The efficiency of a particular threshold is shown versus charged current purity of the sample created for FD Monte Carlo. Each point represents a set of threshold values. The black points represent the efficiency and purity for a given set of thresholds for $\mu \mathrm{ID}-\mathrm{A}$, leaving $\mu \mathrm{ID}-\mathrm{B}$ threshold at 1.0. This is the efficiency-purity curve of $\mu$ ID-A alone, like what was used for the 2008 MINOS $\nu_{\mu}$ disappearance analysis. The use of both $\mu \mathrm{ID}-\mathrm{B}$ and $\mu \mathrm{ID}-\mathrm{A}$ together creates a more complex efficiency versus purity graph given by the red points. The spread of the red points represents the other areas in efficiency-purity space that can be reached by using $\mu \mathrm{ID}$-B alongside $\mu \mathrm{ID}-\mathrm{A}$. $\mu \mathrm{ID}-\mathrm{B}$ effectively extends the reach of the possibly attained values to higher efficiency.

The previous studies have shown where the selection thresholds should be place to maximize the likelihood difference between the oscillation hypothesis and the decay and decoherence hypotheses, maintain the $68 \%$ and $90 \%$

\footnotetext{
${ }^{1}$ Some points prefer absolutely no charged-current selection. However, such a 'selection' would increase the contour in the direction of $\sin ^{2}\left(2 \theta_{32}\right)$ do to the increased contamination from neutral current events and is therefore an unacceptable selection (This was demonstrated in the previous section).
} 


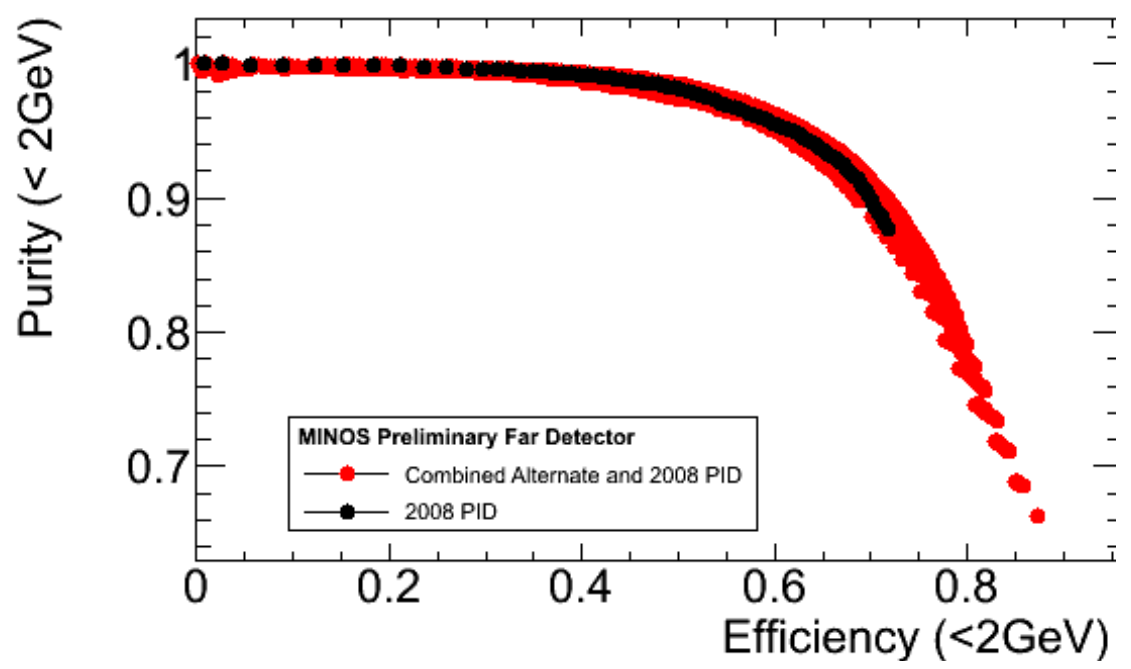

(a) Events with $E_{\nu}<2 G e V$

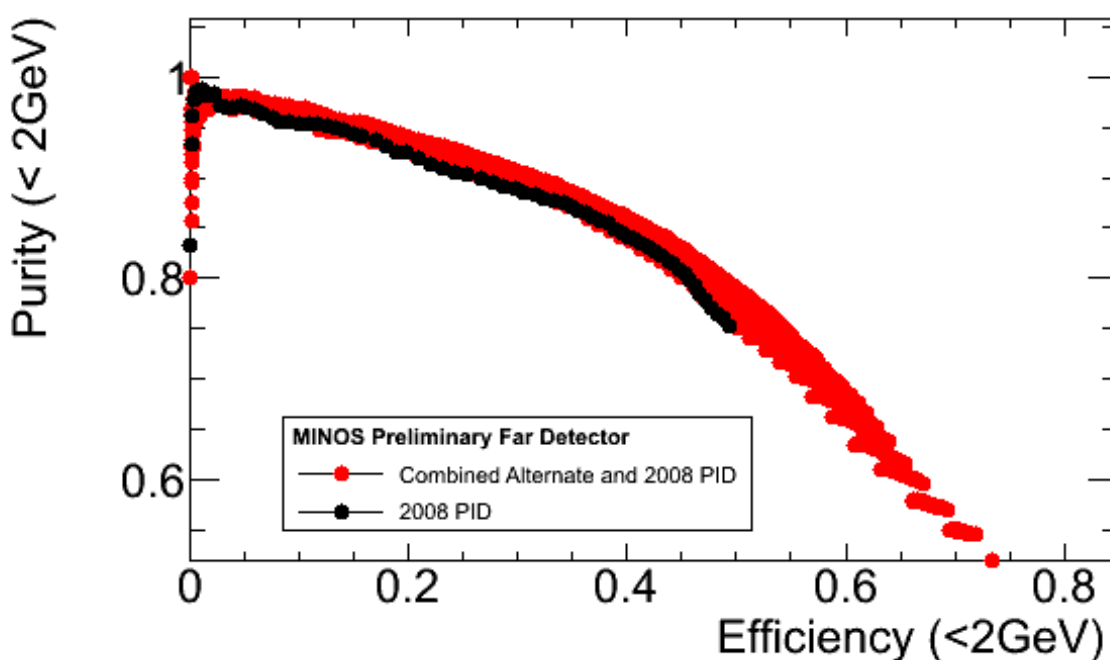

(b) Events with $E_{\nu}<2 G e V$ and \# Planes $<20$

Figure 6.6: The possible values of efficiency and purity for $\mu \mathrm{ID}$-A alone (black), and the combined with $\mu \mathrm{ID}-\mathrm{B}$ (red). Especially at high efficiency, gains can be made without affecting the purity. 
contours of the allowed parameter space, and increase the likelihood of the oscillation hypothesis. However, these studies have not taken into effect how the increase in contaminating neutral current events would affect the systematic errors on the measurement of $\Delta m_{32}^{2}$ and $\sin ^{2}\left(2 \theta_{32}\right)$. How much neutral current contamination is acceptable? Furthermore, $\mu \mathrm{ID}-\mathrm{B}$ has been presented as the only means by which to capture more low-energy charged current events. However, it is possible to 'relax' the threshold on the strong PID from that used in the 2008 MINOS $\nu_{\mu}$ disappearance analysis to a lower value. In fact, $\mu$ ID-A threshold is relaxed between equation (6.1) and equation (6.2). Is $\mu \mathrm{ID}-\mathrm{B}$ necessary?

In Figure 6.7, two plots of the efficiency of the charged current selection versus reconstructed energy are shown with ratio plots to the right. In both plots the threshold values given in equation (6.2), shown in blue, are compared to the thresholds used in the 2008 MINOS $\nu_{\mu}$ disappearance analysis, shown in black. Additionally, a second threshold is compared in red. For the first plot, this red curve represents a 'relaxed 2008 threshold' where $\mu$ ID-B is not used, and the threshold on $\mu \mathrm{ID}$-A is lowered. In the second plot, the red curve represents a 'relaxed $\mu \mathrm{ID}$-A threshold' of $\Theta_{A}=0.2$ and uses a $\mu \mathrm{ID}-\mathrm{B}$ threshold of $\Theta_{B}=0.5$. The efficiency for this combination is higher than the efficiency for the values given in equation (6.2). The ratio of the two colored curves to the black curve representing the 2008 MINOS $\nu_{\mu}$ disappearance analysis is shown on the right of each efficiency plot. The efficiency increases at low energy with use of any of the 'relaxed' thresholds or using $\mu$ ID-B. By relaxing the value of $\Theta_{A}$ to 0.2 from the 0.25 given in equation (6.2), we can increase this efficiency further.

In Figure 6.8 the same event selections as in Figure 6.7 are graphed. 

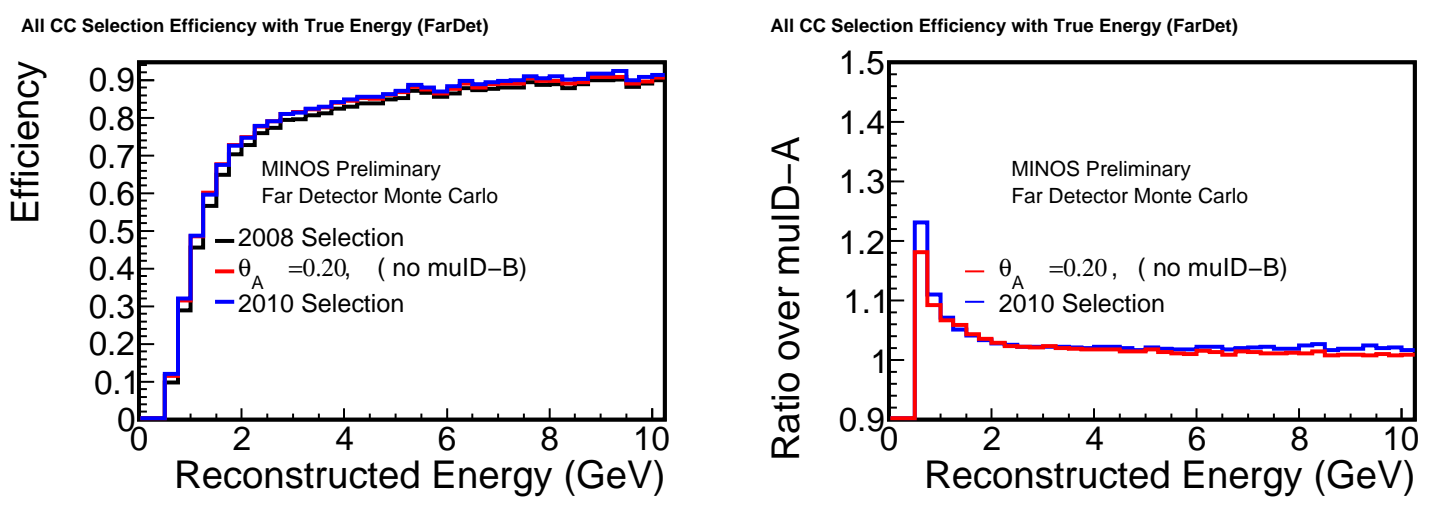

(a)
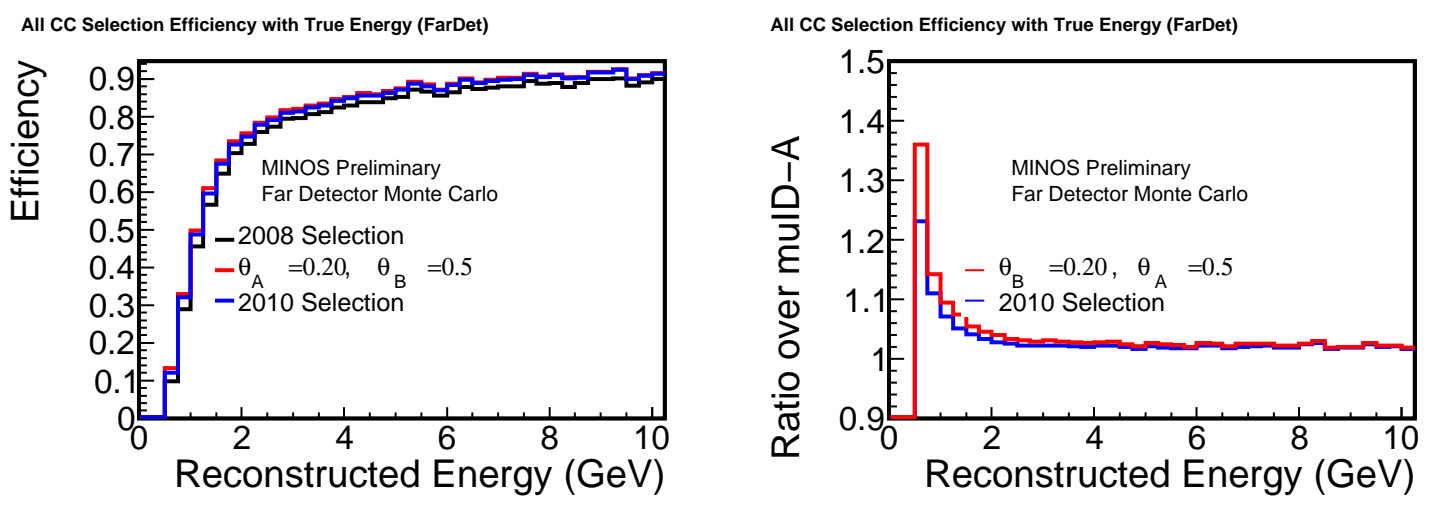

(b)

Figure 6.7: Efficiency versus reconstructed energy for (black) the thresholds given by equation (6.1) and used in the 2008 MINOS $\nu_{\mu}$ disappearance analysis, (blue) the thresholds give in equation (6.2) and proposed for the current analysis, and (red) a 'relaxed' version with $\Theta_{A}=0.2$ and (a) no use of $\mu$ ID-B, or (b) $\Theta_{B}=0.5$. 
However, now the quantity ploted versus reconstructed neutrino is the purity of each sample. By relaxing or introducing new ways to increase our low energy event sample, the purity must suffer, even if just slightly. Therefore, the ratio plots show that the colored curves, which represent selections more relaxed than those used for the 2008 MINOS $\nu_{\mu}$ disappearance analysis, have a lower purity.However, using $\mu \mathrm{ID}-\mathrm{B}$ gives us a more pure sample than just relaxing the threshold $\Theta_{A}$ to a lower value. The blue curve, representing the thresholds given in equation (6.2) are consistently higher (meaning a purer sample) than those give by the red curves, which represent two versions where $\Theta_{A}=0.2$.

In Figure 6.9 the product of the efficiency in Figure 6.7 and purity in Figure 6.8 is the quantity plotted versus reconstructed neutrino energy. Unfortunately, this metric is not useful in predicting what selection thresholds should be used, because it overestimates the importance of efficiency at low energy. Therefore, it strongly prefers very loose selections. However, from the contours seen in Figure 6.4 such extremely loose selections increase the confidence contours in the $\sin ^{2}\left(2 \theta_{32}\right)$ direction.

In order to understand the lower limits the thresholds can have, we studied the neutral current background statistical and systematic error for different thresholds. In Figure 6.10 the neutral current background is shown for four different selections versus the selection used in the 2008 MINOS $\nu_{\mu}$ disappearance analysis in black. In (a) we compare with thresholds values of $\Theta_{A}=0.20$ and $\Theta_{B}=1.0$. In (b), it is compared to the selection defined by $\Theta_{A}=0.25$, and $\Theta_{B}=1.0$. In (c), the 2008 selection is compared to the selection defined by thresholds of $\Theta_{A}=0.2$, and $\Theta_{B}=0.5$. Finally in (d), the 2008 selection is compared to the selection defined by the thresholds $\Theta_{A}=0.25$ and $\Theta_{B}=0.5$, which are the same as in equation (6.2). The Monte Carlo is 

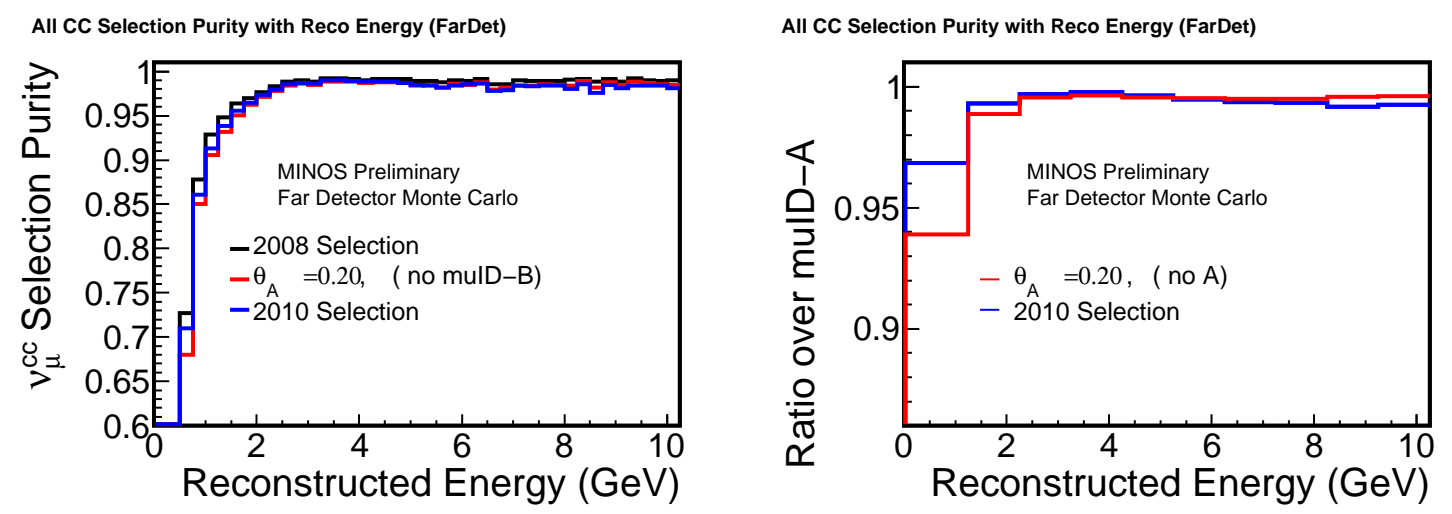

(a)
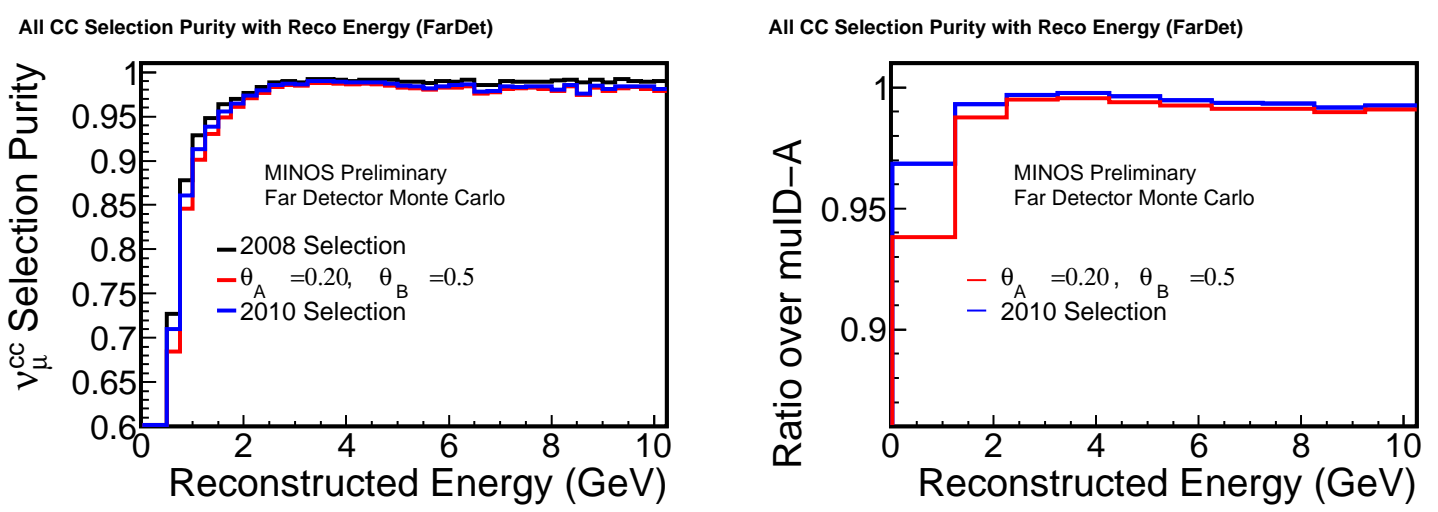

(b)

Figure 6.8: Purity versus reconstructed energy for (black) the thresholds given by equation (6.1) and used in the 2008 MINOS $\nu_{\mu}$ disappearance analysis, (blue) the thresholds give in equation (6.2) and proposed for the current analysis, and (red) a 'relaxed' version with $\Theta_{A}=0.2$ and (a) no use of $\mu$ ID-B, or (b) $\Theta_{B}=0.5$. 

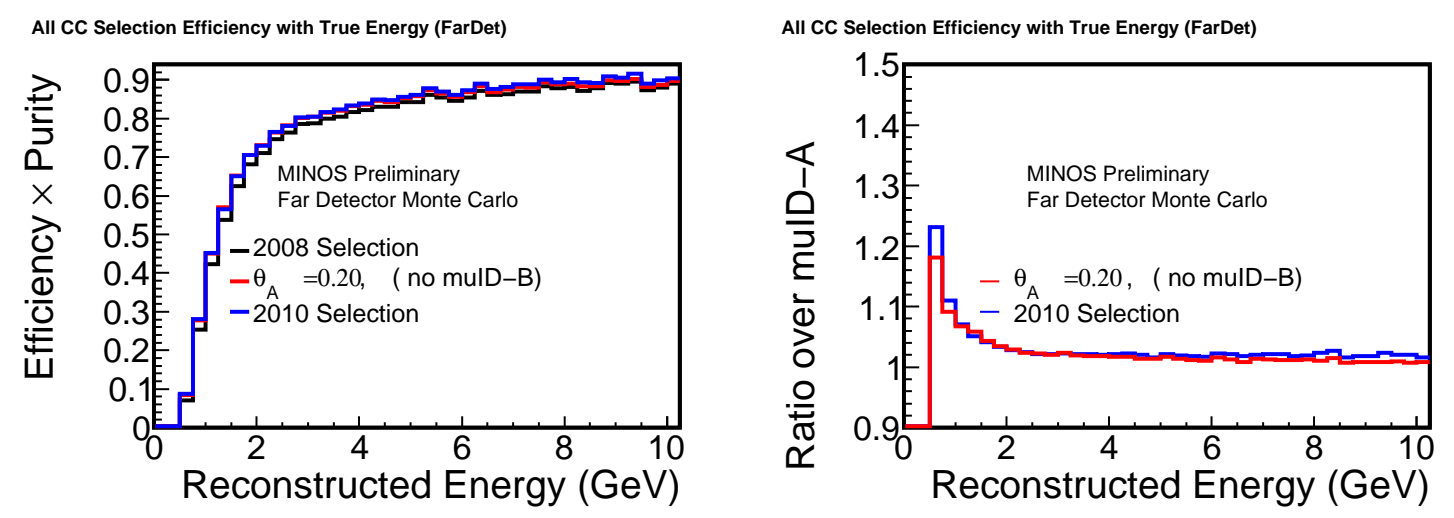

(a)
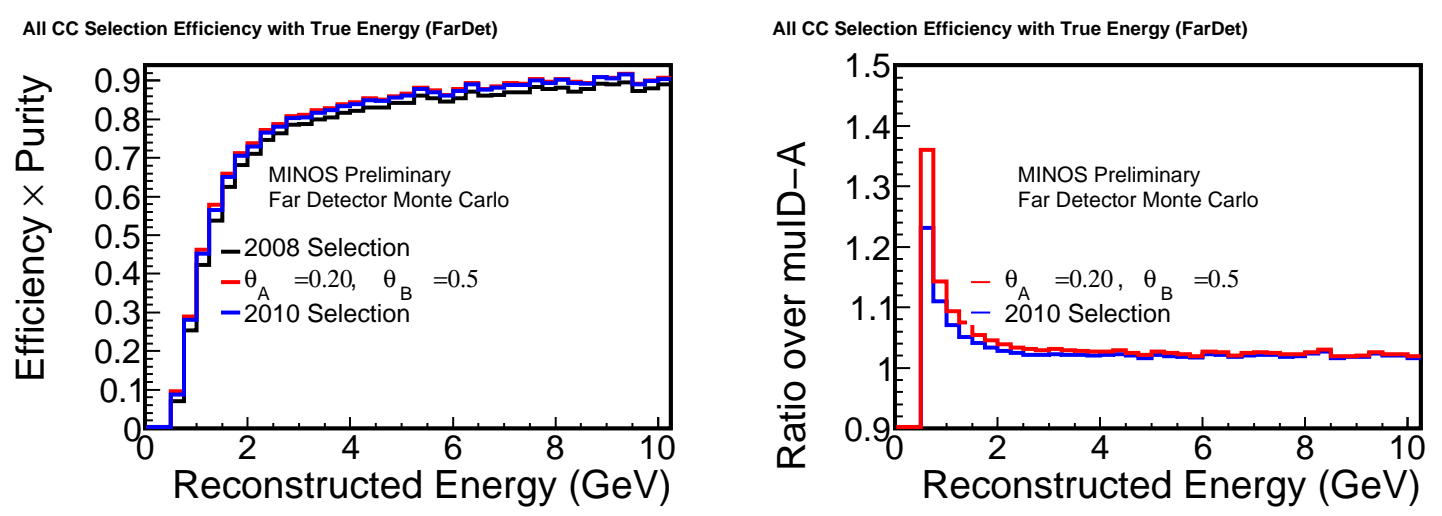

(b)

Figure 6.9: Efficiency times purity versus reconstructed energy for (black) the thresholds given by equation (6.1) and used in the 2008 MINOS $\nu_{\mu}$ disappearance analysis, (blue) the thresholds give in equation (6.2) and proposed for the current analysis, and (red) a 'relaxed' version with $\Theta_{A}=0.2$ and (a) no use of $\mu \mathrm{ID}-\mathrm{B}$, or (b) $\Theta_{B}=0.5$. 
normalized to $7 \times 10^{20}$ POT at the FD, which roughly the statistical size of the current analysis. Additionally, the number of neutral current events for the negative curvature $(Q / P<0) 3.2 \times 10^{20} P O T 2008$ MINOS $\nu_{\mu}$ analysis is displayed on each plot $(9.8 \pm 3.13 \pm 4.9)$. The two errors given for each neutral current background are statistical and systematic respectively. Note that the systematic error for the 2008 analysis was $50 \%$, or 4.9 for 9.8 events. We expect this to drop to $20 \%$ for the current analysis. The studies supporting this will be discussed in Chapter 5 .

Note that (a) and (c) have a lower strong-PID threshold than in (b) and (d) at $\Theta_{A}=0.2$. In fact, (a) and (c) have the same thresholds as the curves in red in Figure 6.7, top and bottom respectively. Therefore, we already know that the charged current selection associated with (a) has a lower efficiency than that associated with (d). Similarly, the charged current selection associated with (b) has a higher efficiency than that of (d). Because (a) has a lower efficiency than (d), but a higher neutral current background (31.7 for (a) versus 31.3 for $(\mathrm{d})$ ), we can remove such threshold candidates. Reducing the strong-PID below 0.2 without using the lowE-PID does not optimize efficiency and purity. (b) displays the smallest change in neutral current background from the 2008 MINOS $\nu_{\mu}$ disappearance analysis levels, however it also has the smallest change in efficiency. Looking at Figure 6.6, such thresholds as used in (a) and (b) of Figure 6.10, which do not make use of the lowE-PID are never optimal for events with less than 20 planes and $3 \mathrm{GeV}$. Furthermore, Figure 6.5(f) shows that the maximum likelihood for oscillations does not lie on the $\Theta_{B}=1.0$ line.

The selection corresponding to those in Figure 6.10 (c) has a high increase in efficiency, as seen in Figure 6.7 and is closer to the best thresholds for 


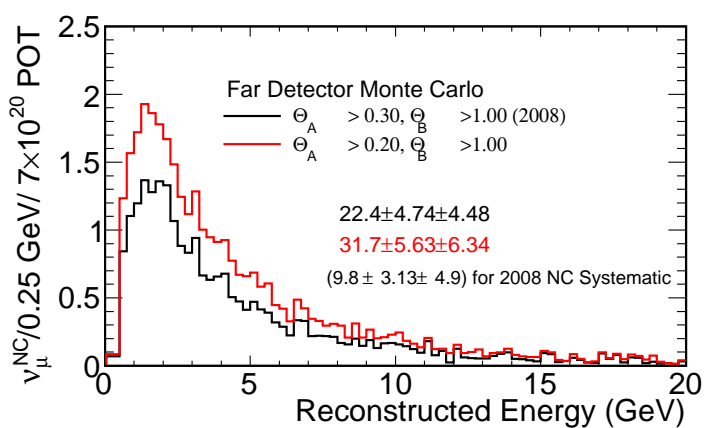

(a) 2008 selection(black), $\Theta_{A}=0.2$, and $\Theta_{B}=1.0($ red $)$

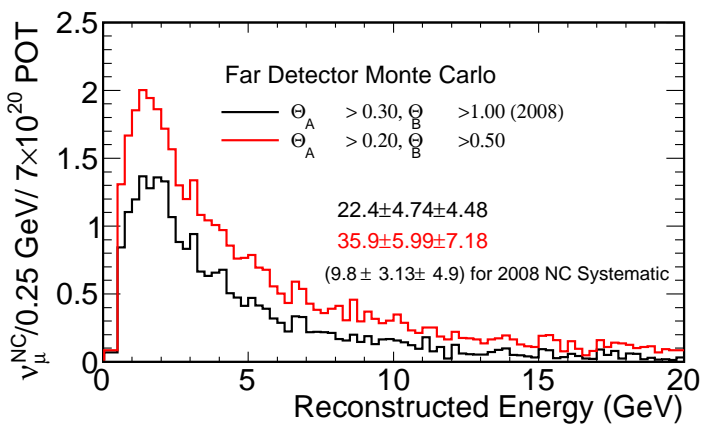

(c) 2008 selection(black), $\Theta_{A}=0.2$, and $\Theta_{B}=0.5$ (red)

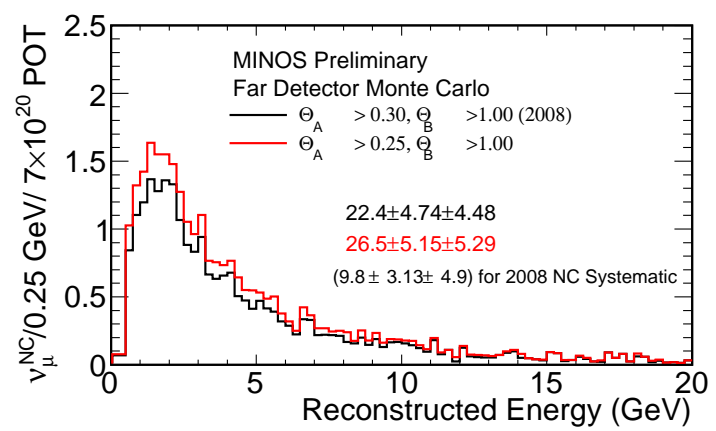

(b) 2008 selection(black),

$\Theta_{A}=0.25$, and $\Theta_{B}=1.0$ (red)

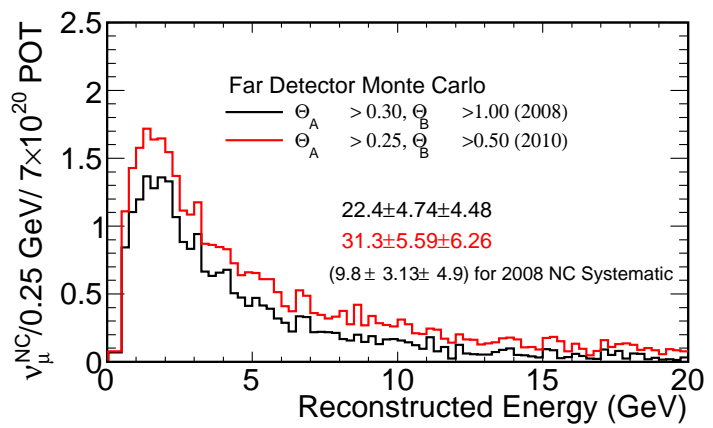

(d) 2008 selection(black),2010 selection (red)

Figure 6.10: The total neutral current background. In each plot the black line represents the neutral current background for the 2008 MINOS $\nu_{\mu}$ disappearance selection. The red curve represents the neutral current background for (a) $\Theta_{A}=0.2$, and $\Theta_{B}=1.0$, (b) $\Theta_{A}=0.25$, and $\Theta_{B}=1.0, \Theta_{A}=0.2$, and $\Theta_{B}=0.5$, and $(\mathrm{c}) \Theta_{A}=0.2$, and $\Theta_{B}=0.5$, which is the selection defined for the current analysis in equation (6.2) 


\begin{tabular}{|c|c|c|}
\hline Point & Statistical + Systematic & Systematic Only \\
\hline (a) & $\frac{\sqrt{\left(5.63^{2}+6.34^{2}\right)}}{\sqrt{\left(3.13^{2}+4.9^{2}\right)}}=1.46=1.03 \times \sqrt{2}$ & $1.29=0.91 \times \sqrt{2}$ \\
\hline (b) & $\frac{\sqrt{\left(5.15^{2}+5.29^{2}\right)}}{\sqrt{\left(3.13^{2}+4.9^{2}\right)}}=1.27=0.89 \times \sqrt{2}$ & $1.08=0.76 \times \sqrt{2}$ \\
\hline (c) & $\frac{\sqrt{\left(5.99^{2}+7.18^{2}\right)}}{\sqrt{\left(3.13^{2}+4.9^{2}\right)}}=1.61=1.13 \times \sqrt{2}$ & $1.47=1.04 \times \sqrt{2}$ \\
\hline (d) & $\frac{\sqrt{\left(5.59^{2}+6.26^{2}\right)}}{\sqrt{\left(3.13^{2}+4.9^{2}\right)}}=1.44=1.02 \times \sqrt{2}$ & $1.27=0.90 \times \sqrt{2}$ \\
\hline
\end{tabular}

Table 6.1: Statistical and systematic errors on the neutral current background for the different thresholds discussed in this section and shown in Figure 6.10 compared to the threshold given in equation (6.1) that was used for a previous analysis [33].We can see that the systematic error does not increase significantly for the different threshold values. The MINOS data sample double since the previous analysis. Therefore, it is useful to remove a factor of $\sqrt{2}$.

maximal liklihood for oscillations (see Figure 6.5(f). However, it was decided that the increases in neutral current background were unacceptably large; the statistics of the current $\nu_{\mu}$ disapperance analysis are twice that of the 2008 analysis ( from 3.2 to $7 \times 10^{20}$ ) giving a $\sqrt{2}$ statistical error reduction. Because MINOS was trying to improve the $\nu_{\mu}$ analysis, it was decided that the systematic error should not increase much above a factor of $\sqrt{2}$, with the absolute upper limit being a 50\% increase in neutral current event error. The total statistical plus systematic neutral current error for the 2008 MINOS $\nu_{\mu}$ disappearance analysis is 5.8 (4.9 systematic). The statistical plus systematic error of each red curve in Figure 6.10 is increased above 2008 levels by the values in Table 6.1

There is a substatial neutral current increase at low energies for the selection of Figure 6.10 (c) which is unacceptable. In contrast, the rise in neutral current for Figure 6.10(d) occurs at higher energies such that the difference in 
neutral current background between Figure 6.10(c) and Figure 6.10(d) below 3 $\mathrm{GeV}$ is very small. This is important because the neutral current background affects the measurement of $\sin ^{2}\left(2 \theta_{32}\right)$ by bluring the depth of the oscillation minimum with more events. We are less concerned with increases in the neutral current background at high energies, where they are a smaller percentage of the events and are far from the oscillation minimum. The thresholds given in equation (6.2) and used in the current analysis are the best values to increase the low energy neutrino sample while keeping neutral current systematic error below a $50 \%$ increase, especially below $3 \mathrm{GeV}$.

Together, the studies in this chapter dictate the threshold values given in equation (6.2). The efficiency and neutral current contamination for the $\mathrm{ND}$ and FD are shown in Figure 6.11 using the thresholds in equation (6.2), which are the thresholds for the event selection used in the current (2010) $\nu_{\mu}$ disapperancea analysis. For the FD, the 2008 efficiency and purity is shown as a dashed line for comparison.

The charged current selection for the 2010 MINOS $\nu_{\mu}$ disapperance analysis measures an increase in the selection efficiency through Monte Carlo by $2.6 \%$ over all energies and by $4.9 \%$ below neutrino energies of $2 \mathrm{GeV}$. The total efficiency is $89.5 \%$ over all energies and $77 \%$ for energies below $2 \mathrm{GeV}$. For these same thresholds, the 2010 MINOS $\nu_{\mu}$ disapperance analysis maintains a neutral current contamination level of $2 \%$ over all energies and $7 \%$ below energies of $2 \mathrm{GeV}$. 


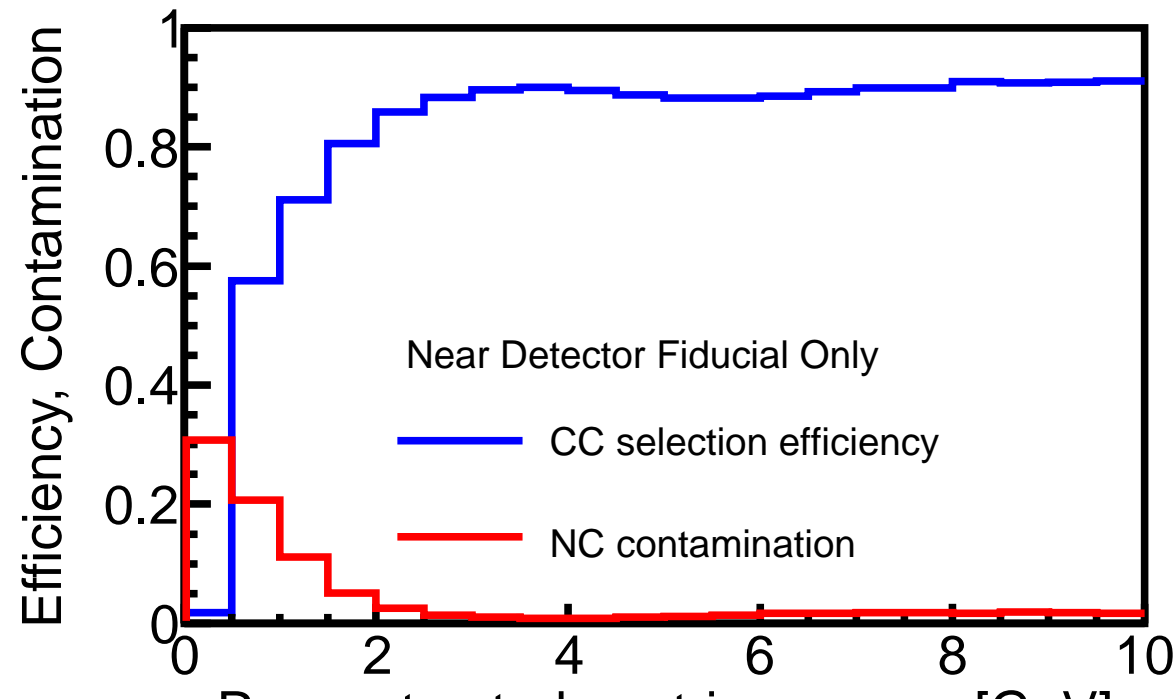

(a) The Rear Detectror Efted neutrincy and energy [ $[\mathrm{GeV}]$ ] neutrino energy

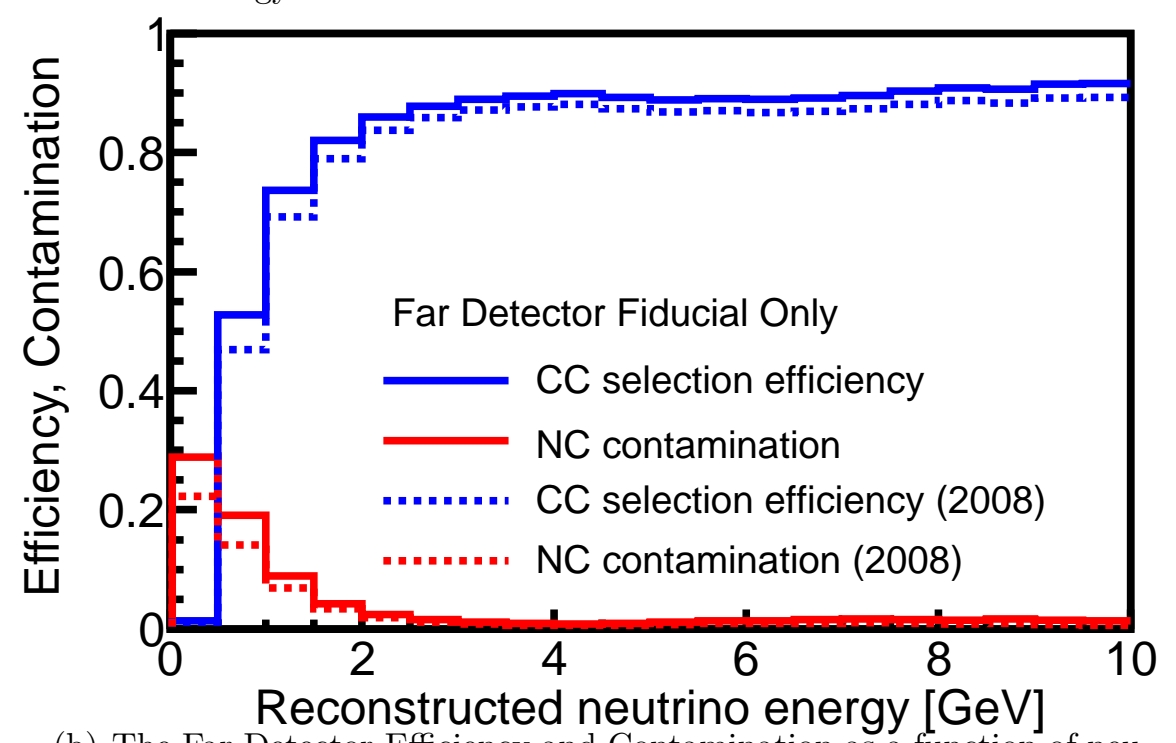

(b) The Far Detector Efficiency and Contamination as a function of neutrino energy

Figure 6.11: Efficiency (blue) and the neutral current contamination fraction (red) of the charged current event selection versus $\nu_{\mu}$ reconstructed enregy in the ND and FD. (a) is the ND and (b) is the FD. The dashed line represents the efficiency and contamination levels given by the threshold values in equation (6.1) and used for the 2008 MINOS $\nu_{\mu}$ disapperance analysis. 


\section{Chapter 7}

\section{Improvements to the MINOS $\nu_{\mu}$ Disappearance Analysis}

The MINOS experiment had been accumulating data for five years when the latest (2010) $\nu_{\mu}$ disappearance analysis was finished. However, a previous measurement of the oscillation parameters through $\nu_{\mu}$ disappearance was published by MINOS in 2008 [33, 83]. Since the 2008 publication, MINOS has doubled the size of its $\nu_{\mu}$ data. The data accumulation for MINOS is summarized in Table 2.1 in prettyrefcha:NuMI. Many of the procedures from the analysis which lead to the 2008 MINOS publication were retained by MINOS for the latest analysis. However, some analysis improvements were made in order to maximize the potential of the increased dataset. In this chapter, we will discuss the results from 2008 MINOS $\nu_{\mu}$ disappearance analysis and the various other analysis improvements that were developed to increase the sensitivity of the current (2010) analysis will be presented.

\subsection{Previous Measurements of Oscillation Parameters Through $\nu_{\mu}$ Disappearance by MINOS}

MINOS published an analysis of the disappearance of muon neutrinos through apparent oscillations in 2008[33]. This was based data accumulated from the MINOS Run I and Run II data series which totals to $3.2 \times 10^{20}$ POT. In contrast to the 2010 analysis discussed in detail in this thesis, this previous 
analysis only used events with negative curvature tracks whose $\mu \mathrm{ID}$-A value surpassed the threshold set in equation (6.1). A 50\% neutral current normalization systematic error was used as opposed to the smaller value suggested by the studies in Chapter 5 .

The results from this measurement are summarized in Figure 7.1. The ratio of the Far Detector reconstructed neutrino energy spectrum with the prediction in the case of no oscillations is shown on the left, while on the right, the contours for the MINOS measurement of the oscillation parameters are compared to those of similar experiments in 2008 and the even earlier MINOS result. The final measurements of the oscillation parameters were $\left|\Delta m_{32}^{2}\right|=(2.43 \pm 0.13) \times 10^{-3} \mathrm{eV}^{2}(68 \%$ confidence level $)$ and mixing angle $\sin ^{2}\left(2 \theta_{32}\right)>0.90(90 \%$ confidence level).

The shifts to the previous best fit oscillation parameters induced by the application of systematic shifts to fake data is shown in Figure 7.1. The total systematic uncertainties are given in Table 7.1 [33].

\begin{tabular}{lll}
\hline \hline Variable Uncertainty & $\delta \Delta m_{32}^{2}\left(10^{-3} \mathrm{eV}^{2}\right)$ & $\delta \sin ^{2}\left(2 \theta_{32}\right)$ \\
\hline Absolute hadronic energy scale $( \pm 10.3 \%)$ & 0.052 & 0.004 \\
Relative hadronic energy scale $( \pm 3.3 \%)$ & 0.027 & 0.006 \\
Normalization $( \pm 4 \%)$ & 0.081 & 0.001 \\
Neutral Current contamination $( \pm 50 \%)$ & 0.021 & 0.016 \\
$\mu$ momentum $($ range $\pm 2 \%$, curvature $\pm 3 \%)$ & 0.032 & 0.003 \\
$\sigma_{\nu}\left(E_{\nu}<10 G e V\right)( \pm 12 \%)$ & 0.006 & 0.004 \\
Beam flux & 0.010 & 0.000 \\
\hline Total Systematic Uncertainty & 0.107 & 0.018
\end{tabular}

Table 7.1: The systematic uncertainties for the 2008 MINOS measurement of the oscillation parameters $\Delta m_{32}^{2}$ and $\sin ^{2}\left(2 \theta_{32}\right)$. 


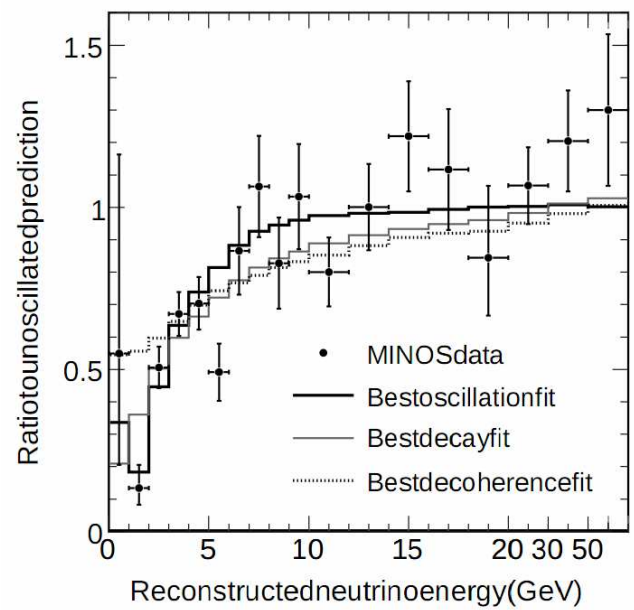

(a)

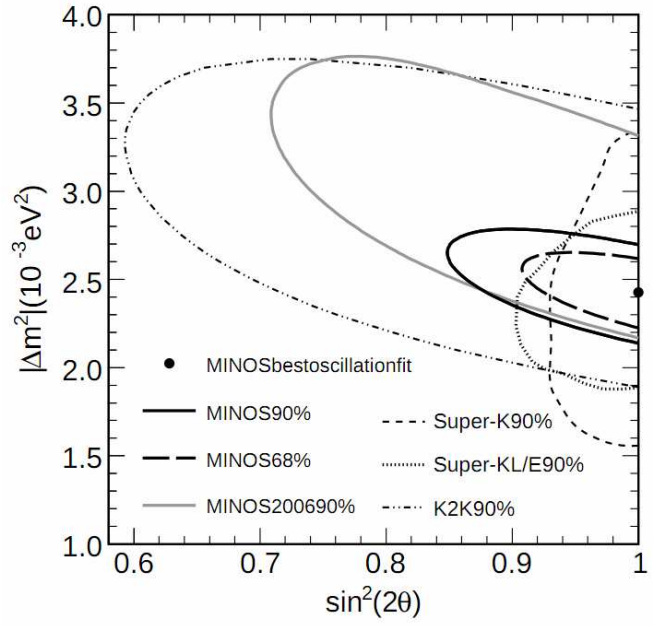

(b)

Figure 7.1: The ratio of the Far Detector data to the unoscillated prediction (a,top) and the contours from the oscillation fit (b,bottom). For (a), the dashed and grey line represent the best fit to the decay and decoherence hypotheses of $\nu_{\mu}$ disappearance. For (b), the results from similar measurements by the SuperK and K2K experiments (fine dashed) [18, 84, 85], and a previous MINOS result [86] ( grey) are compared with the contours derived from a fit to the data shown in (a). 


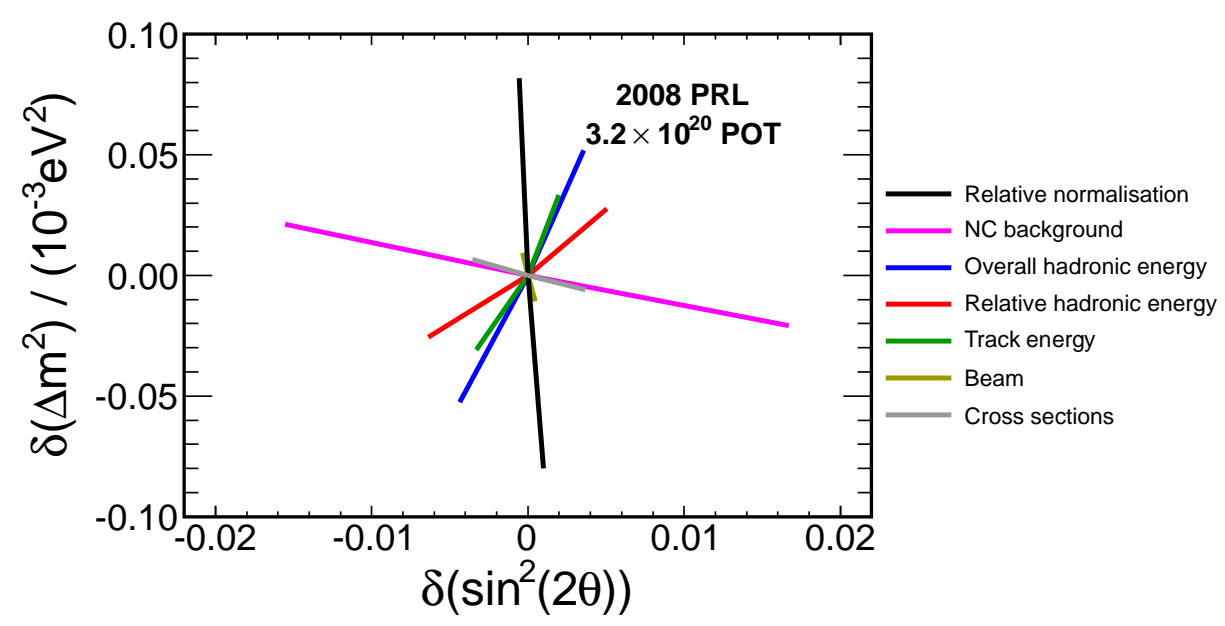

Figure 7.2: The shifts to the best fit oscillation parameters (the fit unconstrained) induced by the application of systematic shifts to the fake data. Shown here are the errors published in 2008 for a smaller exposure $\left(3.2 \times 10^{20}\right.$ POT).

In addition to these measurements of the oscillation parameters, alternative theories to neutrino oscillations through mass eigenstates were considered. Specifically, the MINOS $\nu_{\mu}$ deficit was fit assuming a deficit of neutrinos through neutrino decays into lighter particles and again assuming quantum decoherence without any oscillations. These alternative explanations were disfavored at the 3.7 and $5.7 \sigma$ levels respectively. In Figure 7.1, the best-fit to neutrino decay and neutrino decoherence are shown against the best oscillation fit and the ratio of the observed to expected reconstructed neutrino energy spectrum.

\subsection{Other Analysis Improvements}

The improvement experienced by the current analysis from the previous $2008 \nu_{\mu}$ analysis is mainly due to doubling the MINOS $\nu_{\mu}$ data sample. How- 
ever, the improvements discussed in this section also increased the MINOS's sensitivity to oscillations.

\subsubsection{A k-NN based Shower Energy Estimate}

Previously, the energy of the hadronic shower was reconstructed using a sum of the calorimetric response seen in the MINOS detector. This 'calorimetric energy estimate' does a fair job for showers with energy above 1 or $2 \mathrm{GeV}$. However, at low energies the calorimetric energy estimate deviates from the true energy due to the mismodeling of the intricacies of low-energy multiplicity.

A new energy estimate was developed to address this issue [81]. This energy estimator used a similar k-Nearest-Neighbor algorithm as the one used for the separation of charged current and neutral current events (and detailed in Appendix A). In this case, rather than averaging the identity of the nearby Monte Carlo neighbors, the algorithm averages their true energy. The number of nearest neighbors used in this energy estimate is $k=400$. This value was derived by minimizing the difference between the k-NN shower estimate and the true shower energy. The following variables were used as inputs to the k-NN algorithm

- The number of planes struck by the primary shower

- The sum of all calorimetric shower energy (after various re-weighting schemes)

- The unweighted shower energy near a track vertex ( less than $1 \mathrm{~m}$ )

The purpose of the unweighted shower energy near the track vertex is to weight 
the proportional importance of the number of planes versus the amount of energy seen in each plane.

The results from this analysis are shown in Figure 7.3. The ratio of the $\mathrm{k}-\mathrm{NN}$ shower energy to the true energy is shown versus the true energy of each shower. The k-NN technique does produce a better estimate of the shower energy. However, at low energies, the boundaries of the input distributions produces a strange offset that must be corrected. The offset is fit with a high dimensional polynomial and the k-NN shower energy estimate is weighted by this polynomial to produce a final estimate of the shower energy.

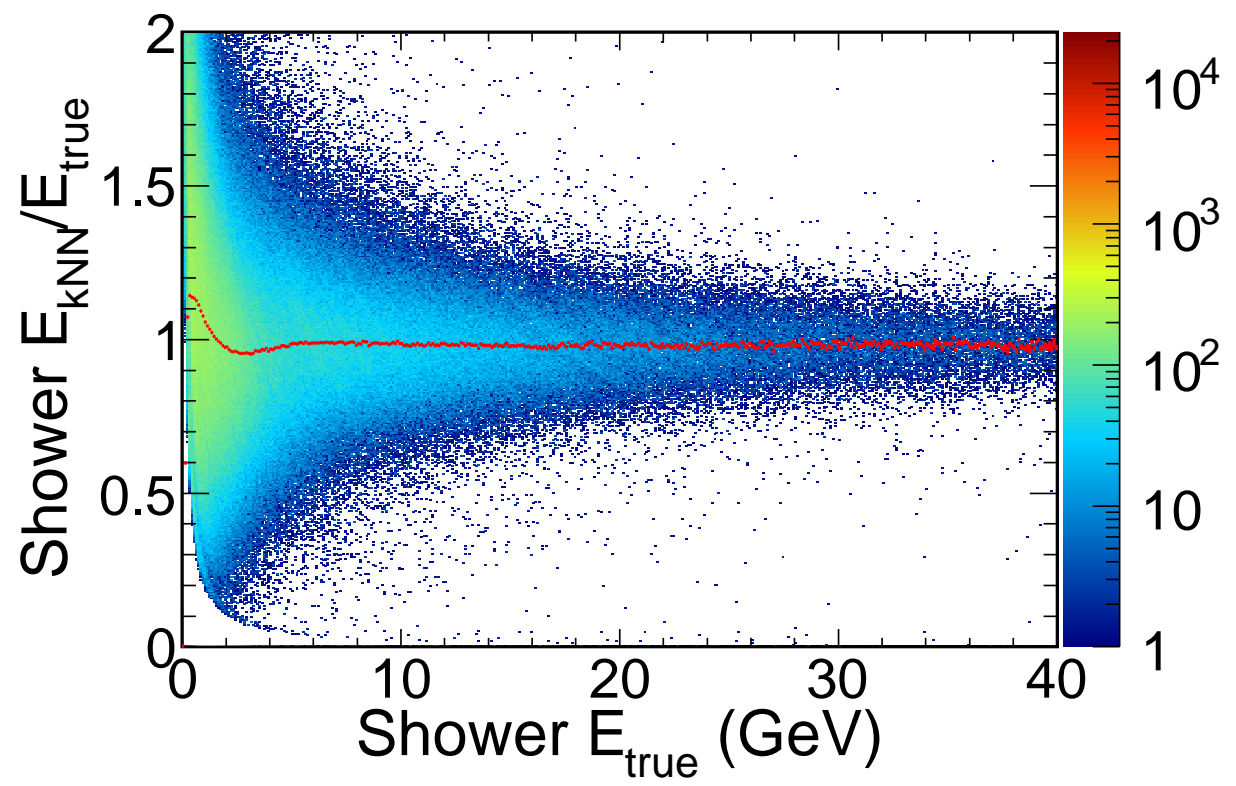

Figure 7.3: The k-NN shower energy estimate before the polynomial correction divided by the true shower energy given as a function of the true shower energy. A red line indicates the polynomial fit to the offsets of the ratio against 1.0. This polynomial fit will be used to correct the k-NN energy estimate and produce a better estimate of the shower energy. 
In Figure 7.4, a Monte Carlo event sample is divided in twelve different true energy bins. The first eleven bins are $0.5 \mathrm{GeV}$ wide such that they encompass showers with energy ranging from 0 to $5.5 \mathrm{GeV}$. The twelfth bin contains showers with energy above $5.5 \mathrm{GeV}$. For each bin, the difference between reconstructed and true shower energies is histogrammed. Both the calorimetric (black) and k-NN (red) shower energy estimates are shown. For each bin, the difference between the k-NN shower energy estimate and the true neutrino energy is much more peaked and centered about the true value (shown as a dashed line). This demonstrates the power of the k-NN shower energy estimate.

The power of this technique can also be seen in the sensitivity contours as shown in Figure 7.5. Here, the k-NN shower energy estimate (before and after correction) is compared to the calorimetric shower energy ("Reco energy") and the limit if the shower energy is known perfectly ("True energy"). There is a significant increase in sensitivity (decrease in the size of the contours) between using the calorimetric and k-NN shower energy estimates.

\subsubsection{The Rock and Anti-Fiducial Analysis}

So far, we have studied only the events that fall within the fiducial volumes of the MINOS detectors. Most fiducial events have contained showers and muons whose energy can be reconstructed well through range or curvature in the magnetic field. However, many events in the MINOS detectors are partially contained, meaning their vertex lies outside the fiducial volume. Such events either have interactions in the MINOS detector but outside the fiducial volume or outside the MINOS detector altogether, usually in the rock walls of the caverns that contain the detector. The goal of this analysis was to 


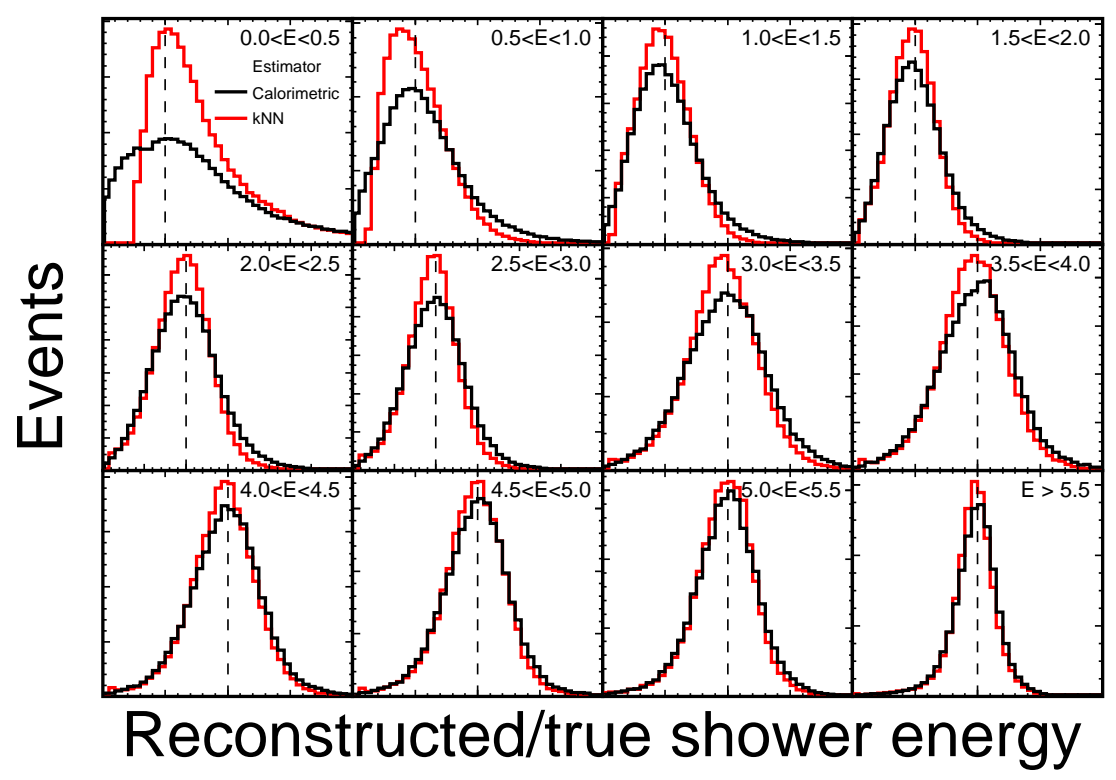

Figure 7.4: Comparison of the calorimetric and k-NN shower energy estimates. The Monte Carlo is divided into twelve bins of true energy. In each sub-figure, the difference between the reconstructed and true shower energy is shown as a histogram. The black histograms represent the calorimetric energy estimate and the red histograms represent the k-NN shower energy estimate. 


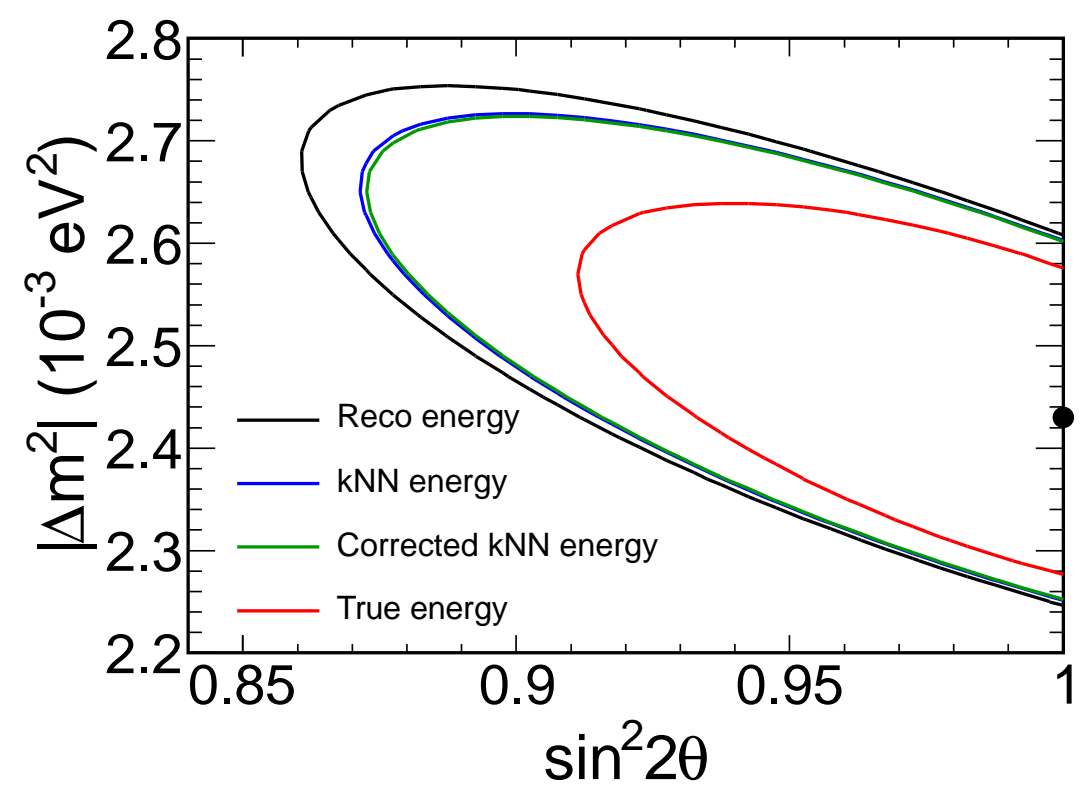

Figure 7.5: The sensitivity to oscillations given various different shower energy estimates. The calorimetric shower energy estimate is shown in black; the kNN shower energy estimates are shown in blue (before correction) and green (after correction); and the true energy (absolute limit) is shown in red. 
increase our statistical sample size by including the 'Rock and Anti-fiducial' (RAF) event sample [87].

The various regions of the 'anti-fiducial' volume are highlighted in Figure 7.6. The RAF sample can be further categorized by the location reconstructed vertex of the events. The sub-samples tend to be dominated by either rock or anti-fiducial events. In particular, events with a reconstructed vertex in the front face (shown in red in Figure 7.6) are mostly neutrino events from the rock upstream of the MINOS Far Detector. Conversely, events reconstructed in the back of the detector (shown in orange in Figure 7.6) are anti-fiducial events where the true vertex is within the MINOS Far Detector, but the event is not well contained. Events which are reconstructed at the sides of the MINOS Far Detector ( shown in light purple in Figure 7.6 are an even mixture of rock events and anti-fiducial detector events. Finally, events with vertices in the region of the super-module gap of the Far Detector (shown in dark purple) are mostly anti-fiducial detector events although near the edges, the number of rock events increases (shown in blue).

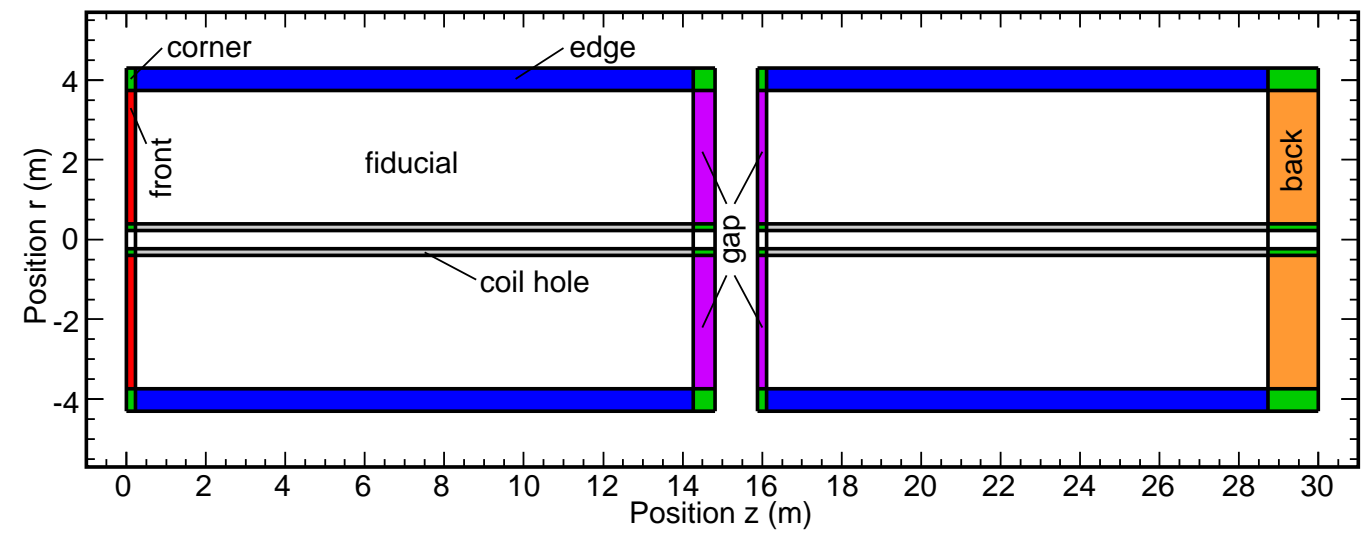

Figure 7.6: The anti-fiducial regions of the Far Detector. 
Although the RAF events from the side of the MINOS Far Detector are an even mixture of anti-fiducial detector and rock events, MINOS can predict whether a particular event is from the anti-fiducial or rock regions by how far from the edge of the detector the reconstructed vertex lies. Rock events tend to have reconstructed vertices at the very edge of the detector. Antifiducial detector events tend to have vertices evenly distributed throughout the anti-fiducial region. The separation between rock and anti-fiducial detector events is important because the two samples have extremely different energy distributions due to the kinematics of the muon from the charged current event; for anti-fiducial detector events the muon easily travels through the detector, but muons from rock events have a particular selection due to the position of the detector far from the edges of the cavern.

In Figure 7.7, the true neutrino energy for rock and anti-fiducial detector events selected as part of the final RAF sample is shown. Rock events have a much larger high-energy component and compose a large fraction of the RAF sample. The anti-fiducial detector event energy spectrum more closely resembles the fiducial energy spectrum. The RAF analysis selects charged current events using the same $\mu \mathrm{ID}-\mathrm{A}$ and $\mu \mathrm{ID}-\mathrm{B}$ described previously. However, because the RAF sample differs significantly from the fiducial sample the thresholds were optimized independently. For detector-like events the thresholds used matches the thresholds in equation (6.2). For rock-like events, the thresholds are lowered to $\Theta_{\text {strong }}=0.09$ and $\Theta_{\text {lowE }}=0.02$. For events which are detector-like but are reconstructed at the back of the Far Detector, the threshold cuts were changed to $\Theta_{\text {strong }}=0.14$ and $\Theta_{\text {lowE }}=0.01$. This last threshold cut essentially allows all events with a track to pass. These thresholds were found by optimizing for the best sensitivity to $\Delta m_{32}^{2}$ for RAF events. 


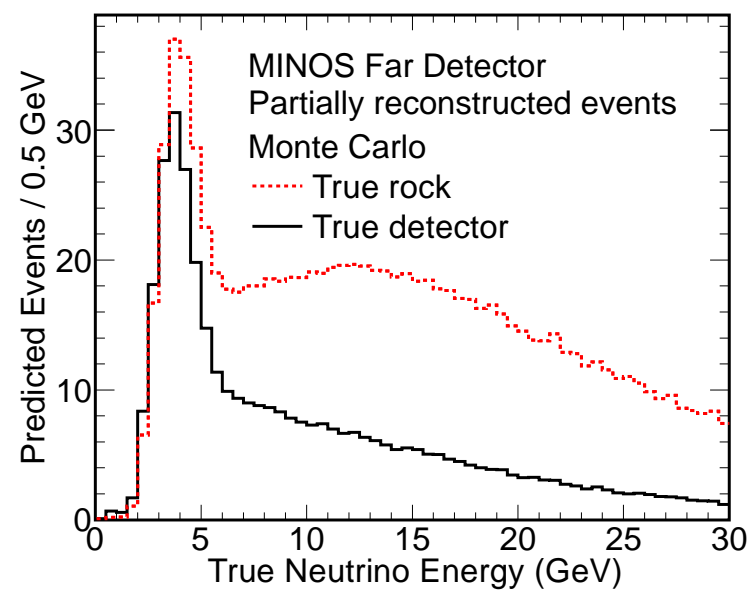

Figure 7.7: The true neutrino energy spectrum for anti-fiducial detector events (black) and rock events (red). This is composed of events which are part of the final RAF selection.

It should be noted that because of the low neutral current background the threshold values have little impact on the final RAF sample.

There is, however, a serious issue with including RAF events in the $\nu_{\mu}$ disappearance analysis. The shower energy for RAF events is not wellcontained. Therefore, large shower energy uncertainties exist and the neutrino energy estimate uses only the reconstructed muon energy. In Figure 7.8, the reconstructed muon energy is compared to the true neutrino energy for anti-fiducial detector events and rock events. The reconstructed muon energy merely provides a lower limit of the true neutrino energy. Therefore, the energy-dependent shape of the neutrino deficit cannot be resolved with RAF events.

Nevertheless, RAF events can be used by the oscillation analysis to improve statistics at high-energy where MINOS does not expect to see a large deficit and observing an energy-integrated deficit of neutrinos. The effect of 

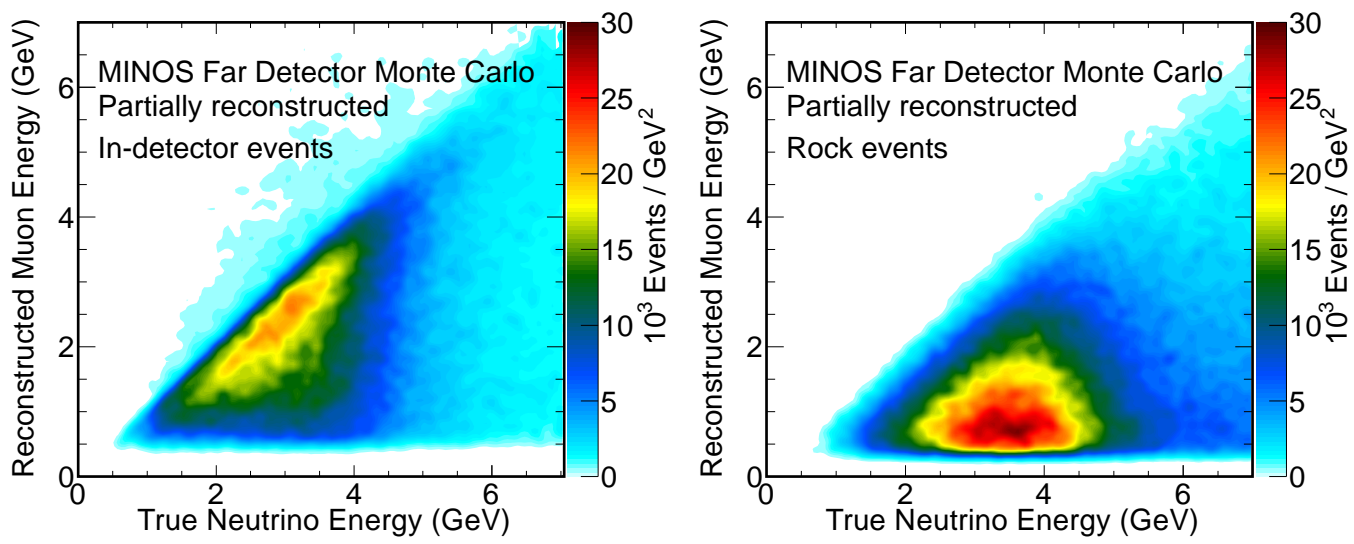

Figure 7.8: Comparison of true neutrino energy to the reconstructed muon energy for anti-fiducial detector events (left) and rock events (right)

including RAF events in the measurement of $\sin ^{2}\left(2 \theta_{32}\right)$ and $\Delta m_{32}^{2}$ is shown in Figure 7.9. Performing an analysis with RAF events in parallel with the fiducial $\nu_{\mu}$ disappearance measurement reduces the contours for both parameters but especially for $\Delta m_{32}^{2}$.

\subsubsection{Resolution Binning}

As was mentioned previously, the energy resolution of an event is better for some events than for others. Events with poor energy resolution (like RAF events) can the energy-dependent oscillation signal. This is why the RAF events are not directly added to the fiducial sample.

Fiducial events can also vary from being well resolved to poorly resolved. The estimated error of an event's reconstructed energy is given as

$$
\sigma_{\text {total }}=\sqrt{\sigma_{\text {track }}^{2}+\sigma_{\text {shower }}^{2}} .
$$

where $\sigma_{\text {track }}$ and $\sigma_{\text {shower }}$ are the uncertainty on the track and shower energy respectively. For track energy estimates through range, $\sigma_{\text {track }}^{2}$ is proportional 


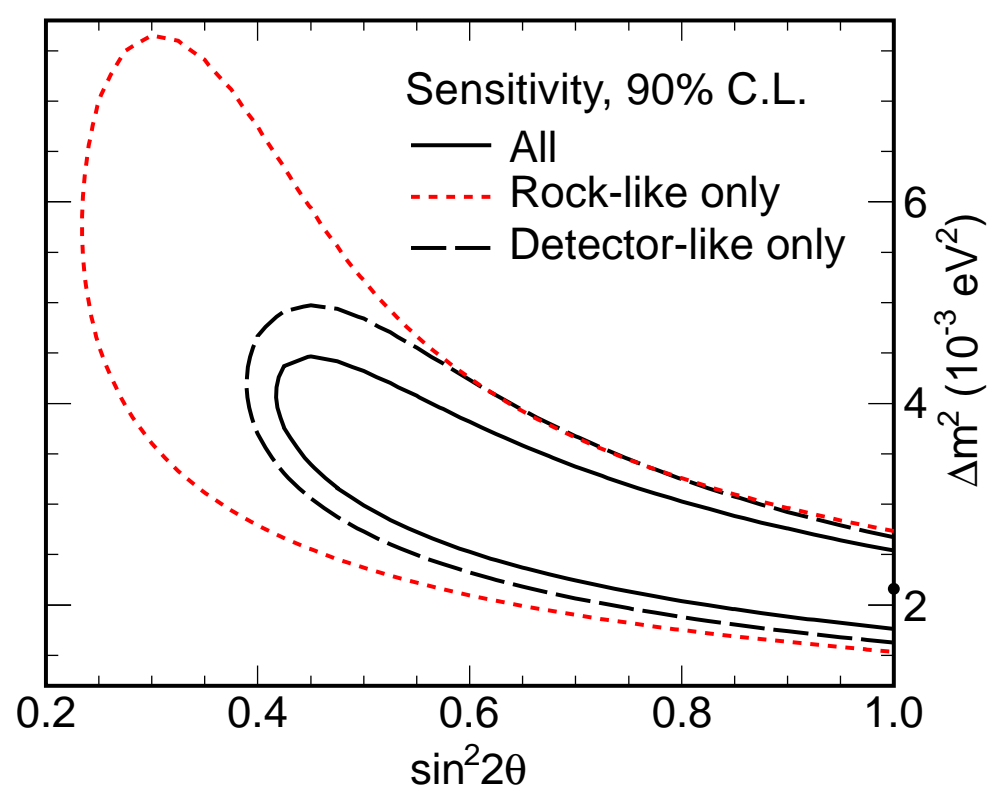

Figure 7.9: The sensitivity of the $\nu_{\mu}$ oscillation analysis using only fiducial events(dashed black curve), RAF events (dotted red curve), and combined (solid black line). Adding the RAF to the fiducial event analysis reduces the contours. 
to the track energy squared and for track energy estimates through curvature, $\sigma_{\text {track }}$ is proportional to the error of the track fit. $\sigma_{\text {shower }}^{2}$ is proportional to the shower energy estimate squared. The track (by range) and shower energy uncertainty parametrizations are shown in Figure 7.10.

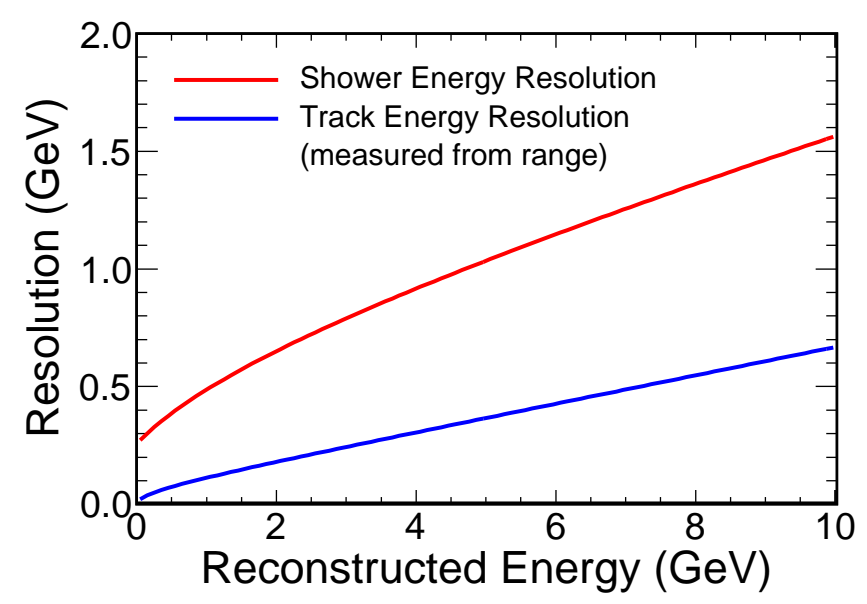

Figure 7.10: The resolution parametrization for tracks by range and shower energy. For a given event the shower energy uncertainty and track energy uncertainty are added in quadrature to produce the overall uncertainty in the event's energy.

In the $2010 \nu_{\mu}$ disappearance analysis, MINOS expanded the concept of separating low-resolution events from separating RAF from fiducial events to separating the fiducial sample into independent 'resolution bins' [58]. The resolution of each event is given by $\sigma_{\text {total }} / E_{\text {reco }}$ and the events were divided using this parameter into five resolution bins, or qunitiles, with equal integral number of events. The indices of these five bins range from zero to four. The resolution parameter is shown versus the reconstructed energy in Figure 7.11.

A sixth 'resolution bin' contains all events with positive curvature 
tracks even though such events are likely $\overline{\nu_{\mu}}$ interactions, which have been considered a source of contamination in the past. Low-energy $\nu_{\mu}$ events with short muon tracks often have a mis-identified charge-sign due to the small number of hits through which the reconstruction can measure the curvature. Including positive curvature events allows MINOS to reclaim such low-energy events. Positive curvature events were not included previously because of the fear of contaminating the $\nu_{\mu}$ sample. With the addition of resolution binning, MINOS can safely use positive curvature events in the fit without the risk of contaminating the entire $\nu_{\mu}$ event sample. For the small running in the pseudo-high energy configuration positive curvature events are still excluded.

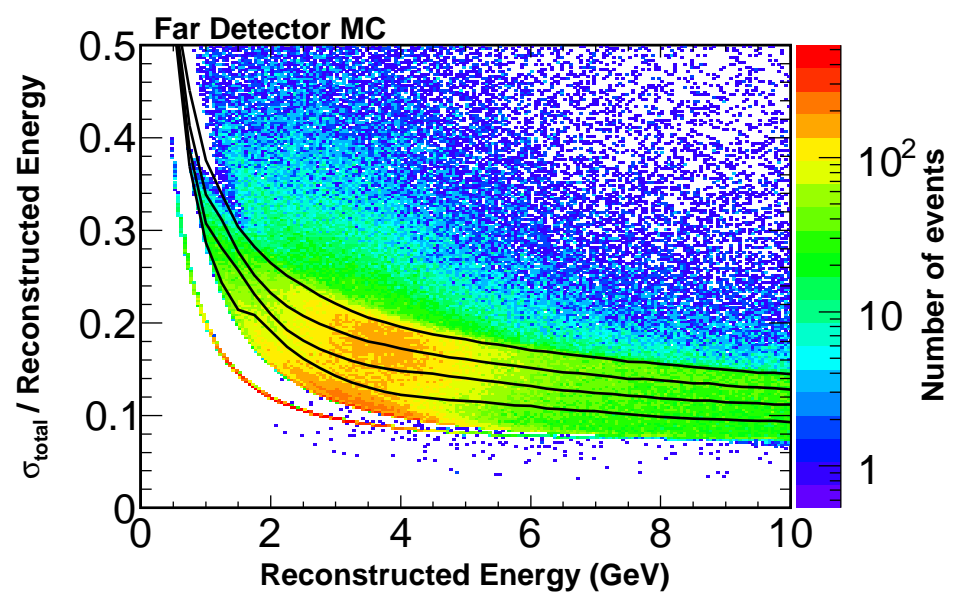

Figure 7.11: Resolution is parametrized using track energy, shower =energy, and track containment variables. Cuts are then trained as a function of reconstructed energy to define five quantiles that yield identical unoscillated predicted spectra, while retaining different energy resolutions. The black lines show resulting four cuts that divide these five regions.

In Figure 7.12, the reconstructed energy is shown versus the true energy 


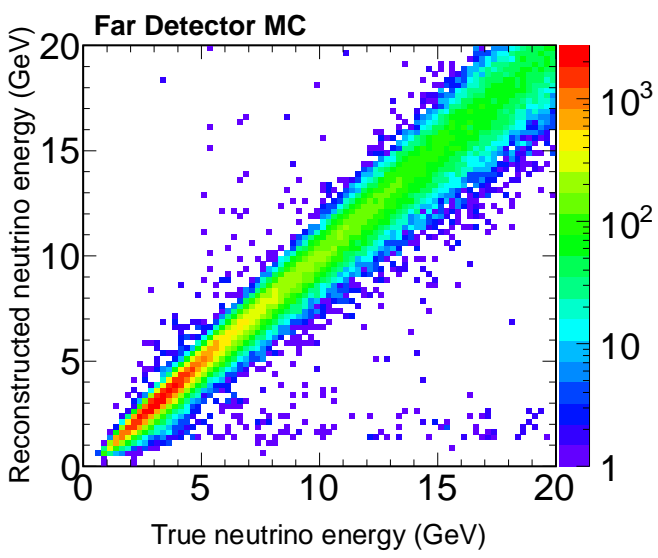

(a) $\operatorname{Bin} 0$

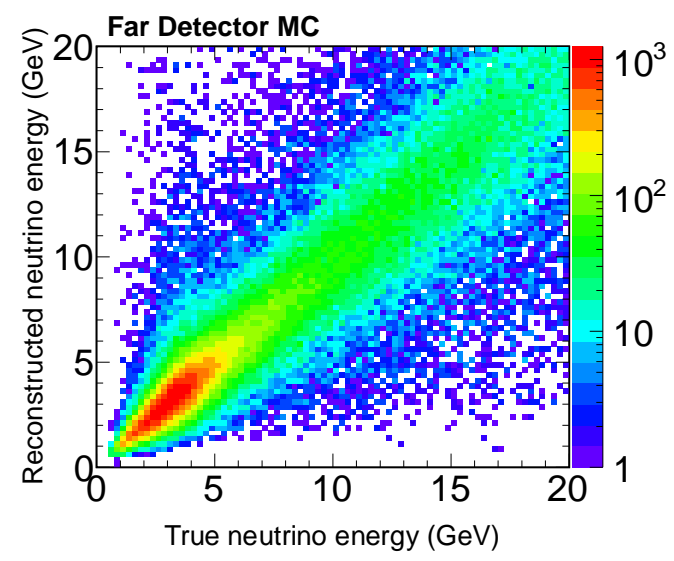

(b) $\operatorname{Bin} 4$

Figure 7.12: The reconstructed energy versus the true energy for events in (a) The highest resolution bin and (b) the lowest resolution bin.

for Monte Carlo events in the zeroth and fourth bin. The zeroth bin events have reconstructed energy which deviates much less form the true energy than events in the fourth bin. This is the power of the resolution binning method. By fitting each bin separately, the high-resolution events have the accuracy to resolve the position and depth of the oscillation minimum whereas the lowresolution events aid in the overall statistical

\subsection{Summary of the Analysis Improvements}

The analysis improvements described in this section allow MINOS to improve its sensitivity to the oscillation parameters beyond the base improvement due to the larger sample. The use of these analysis improvements compared to the earlier "2008 analysis" is shown in Figure 7.13. The resolution 
binning and k-NN shower energy estimate mainly increase the sensitivity to $\sin ^{2}\left(2 \theta_{32}\right)$ whereas including the RAF analysis increases the sensitivity to the mass splitting, $\Delta m_{32}^{2}$.

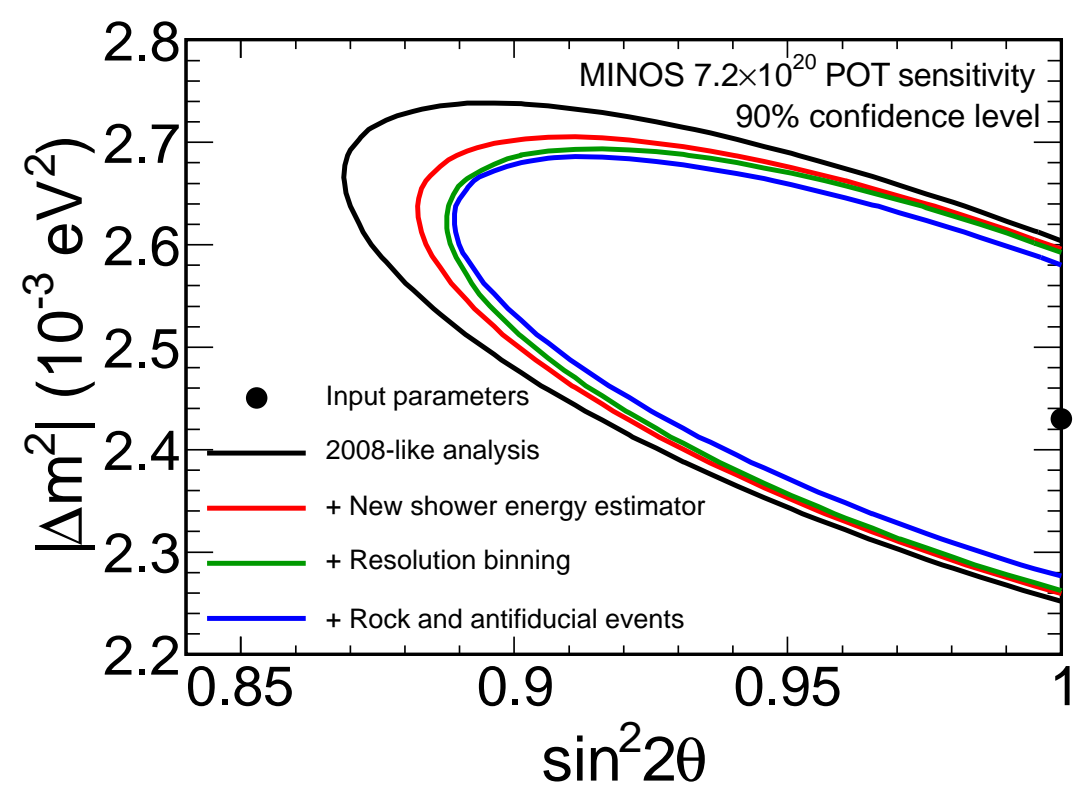

Figure 7.13: The changes in sensitivity produced by shower energy estimator, resolution binning and inclusion of rock and anti-fiducial events. Each analysis improvement reduces the allowable space of the oscillation analysis. 


\section{Chapter 8}

\section{Results from the 2010 MINOS $\nu_{\mu}$ Disappearance Analysis}

MINOS began analyzing the combined Run I through Run III Far Detector data after the procedures and improvements described in the previous chapters were set. In this chapter, the final calculation of systematic errors and distributions before the unblinding and the results from this $\nu_{\mu}$ disappearance analysis will be reviewed. This includes the measured values of the oscillation parameters $\Delta m_{32}^{2}$ and $\sin ^{2}\left(2 \theta_{32}\right)$ and increases of the disfavorment of decay and decoherence hypotheses.

\subsection{Calculation of Systematic Errors}

The systematic errors for the oscillation measurement are summarized in Table 8.1. The shifts in best fit values of $\Delta m_{32}^{2}$ and $\sin ^{2}\left(2 \theta_{32}\right)$ given shifts the assumed values of variables which contribute to the systematic error can be shown graphically as in Figure 8.1. These shifts can be compared to the shifts for the previous analysis (and smaller exposure) shown in 7.1.

The primary contributions to the systematic error on the oscillation parameters include:

- Neutral Current Background (20\%) - This uncertainty was discussed extensively in Chapter 5. 
- Normalization (1.6\%). This error primarily arises from a $1.3 \%$ selection bias between the Near and Far Detectors. This was determined by a visual scan of events [88]. However, some of this error arises from uncertainty in the thickness of the steel plates of the Near Detector and Far Detector which results in fiducial mass uncertainties.

- Shower Energy Normalization- This uncertainty is energy dependent. It includes a 5.7\% energy independent uncertainty due to hadronic energy calibrations as well as an energy dependent error band derived from NEUGEN. The NEUGEN errors are concerned with nuclear effects for which there is little data and not well-modeled. This is especially true at low inelasticity. This error was evaluated with the calorimetric shower energy. The k-NN shower energy uses the calorimetric energy as an k-NN input and, therefore, the error is still relevant.

- Near and Far Detector Energy Estimation (1.9\% and 1.1\% respectively) - This represents the uncertainty between the observed and simulate calorimetric response relative to the absolute shower energy scale (whose error is described in the last bullet). The systematic error is described in the MINOS position paper by Jeff DeJong[89].

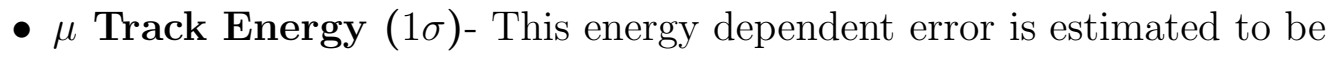
a $2 \%$ uncertainty in the muon momentum if the momentum was taken from the range of the particle and $1 \%$ uncertainty if the muon momentum was taken from curvature relative to the range error.

- Beam Parameterization( $(1 \sigma)$ - This is the error associated with various uncertainties in the beam including target position, beam composition, and cross section. 
- $\bar{\nu}_{\mu}$ charge sign (40\%)- This error estimates the uncertainty in the $\nu_{\mu}$ fraction of positive curvature events (and generally the composition of positive curvature events). The error was estimated from the largest data-Monte Carlo discrepancy of the fraction of $\nu_{\mu}$ positive curvature events. This maximal discrepancy occurs at higher energy and is therefore a conservative estimate of the effect and size of the data-Monte Carlo disagreement. This error was studied by Justin Evans [90].

- Neutrino cross sections- The neutrino cross section is not well known at MINOS energies, as discussed in Chapter 1. This produces an uncertainty in the total number of neutrino events at a given energy independent of the flux uncertainty. Furthermore, uncertainties in the exclusive cross sections lead to uncertainties in the relative rates of various kinds of neutrino interactions.

- Rock Normalization (0.9\%)- With the introduction of partially contained events in the MINOS $\nu_{\mu}$ analysis new errors arise. These errors are related to the composition of the rock surrounding the Far Detector. The percent error on the number of expected rock events can be estimated through the average Z/A ratio of the surrounding rock. This and the following two uncertainty were estimated by Matthew Strait [87].

- Rock cross-section (1\%)- The cross section experienced by neutrinos interacting in the rock surrounding the Far Detector is also affected by the density of the rock. This was estimated to be a $1 \%$ error by Matthew Strait.

- Detector Edge $(1 \sigma)$ - The uncertainty in the placement of the edges of the detector. The strips are shifted 2.5 to $10 \mathrm{~mm}$ with a Gaussian 


\begin{tabular}{l|ccc} 
Variable & Uncertainty & $\delta\left(\Delta m_{32}^{2}\right)$ & $\delta\left(\sin ^{2}\left(2 \theta_{32}\right)\right)$ \\
\hline Shower Energy & $1 \sigma$ & 0.051 & 0.000 \\
Relative Shower Energy & $1.9 \% / 1.1 \%$ & 0.006 & 0.004 \\
Normalization & $1.6 \%$ & 0.041 & 0.000 \\
Neutral Current Background & $20 \%$ & 0.005 & 0.009 \\
$\mu$ Momentum & $2 \% / 3 \%$ & 0.048 & 0.001 \\
$\sigma_{\nu}$ (sum in quadrature) & $1 \sigma$ & 0.017 & 0.006 \\
Beam & $1 \sigma$ & 0.014 & 0.001 \\
$\bar{\nu}_{\mu}$ wrong-sign & $30 \%$ & 0.002 & 0.002 \\
RAF only & $1 \sigma$ & 0.006 & 0.000 \\
\hline Total & & 0.085 & 0.012
\end{tabular}

Table 8.1: Systematic errors and the shifts in best fit values of $\Delta m^{2}$ and $\sin ^{2}(2 \theta)$ they induce. Relative shower energy is $1.9 \%$ ND, $1.1 \%$ FD. $\mu$ momentum is $2 \%$ range, $3 \%$ curvature. Other $1 \sigma$ shifts are either energy dependent or uncorrelated sums of several errors. This includes both Fiducial and RAF event samples

\section{distribution.}

Note that the items listed with a ' $1 \sigma$ ' deviation have an energy dependent systematic error. In calculating the shifts to the oscillation parameters due to the systematic error, a $1 \sigma$ shift of the parameter is used.

In addition to affecting the measurement of the oscillation parameters, systematic errors also affect MINOS's ability to select among a variety of different models of neutrino oscillations. The systematic errors are still present in fits to alternative models and affect the $\chi^{2}$ minimum and shape.

Pure decay and decoherence have been disfavored by the MINOS experiment previously [31-33]. The current MINOS $\nu_{\mu}$ disappearance analysis uses the size of this disfavorment to measure the strength of the neutrino oscillation hypothesis over other models of neutrino disappearance. The discrimination between these alternative hypotheses and oscillations is given in terms of the 


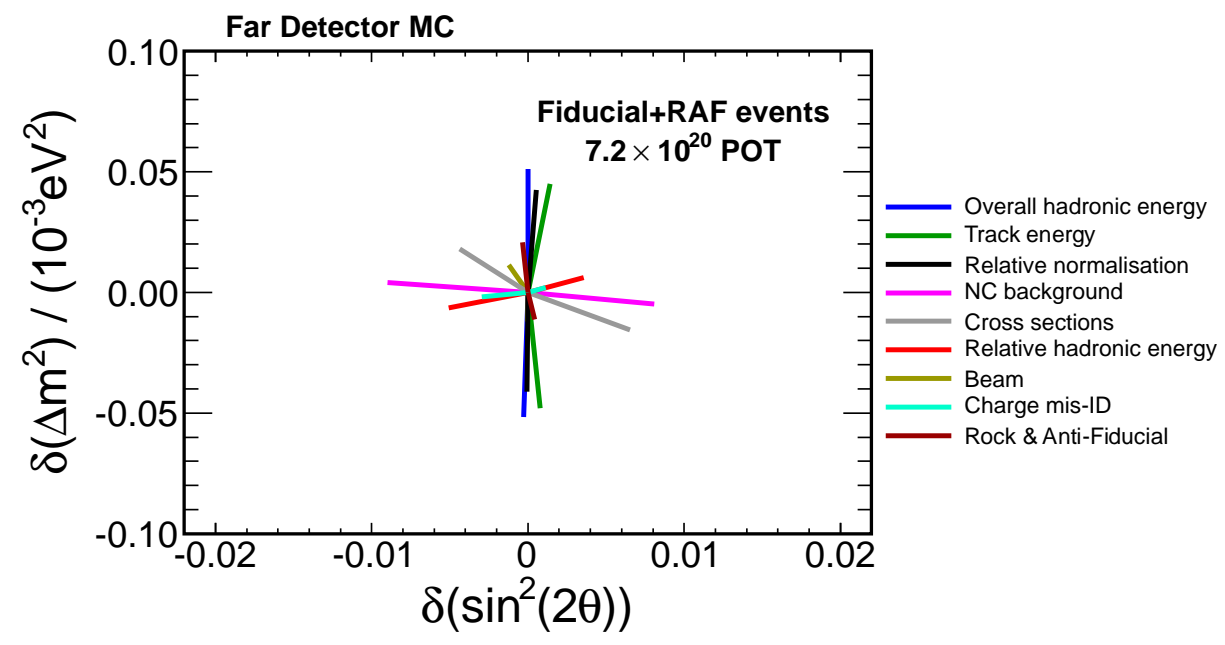

Figure 8.1: The shifts to the best fit oscillation parameters (the fit unconstrained) induced by the application of systematic shifts to the fake data. Shown are are shifts to the fit of fiducial events and RAF events.

difference in $\chi^{2}$. Specifically

$$
n \sigma=\sqrt{\chi_{\text {model }}^{2}-\chi_{\text {oscillations }}^{2}}
$$

This method can be used because the value of $\chi^{2}$ for the best fit oscillations is near zero. The total systematic error effect on the decay and decoherence can be seen in Figure 8.2.

\subsection{The Fitting Procedure}

The introduction of resolution binning and the RAF and positive curvature event samples imposes new conditions on the fitting algoritm. Instead of fitting a single Far Detector $\nu_{\mu}$ distribution, the fitting algorithm must maximize the log-likelihood for five to seven (including RAF and the positive curvature event sample) distributions at the same time [58, 92, 93]. There- 


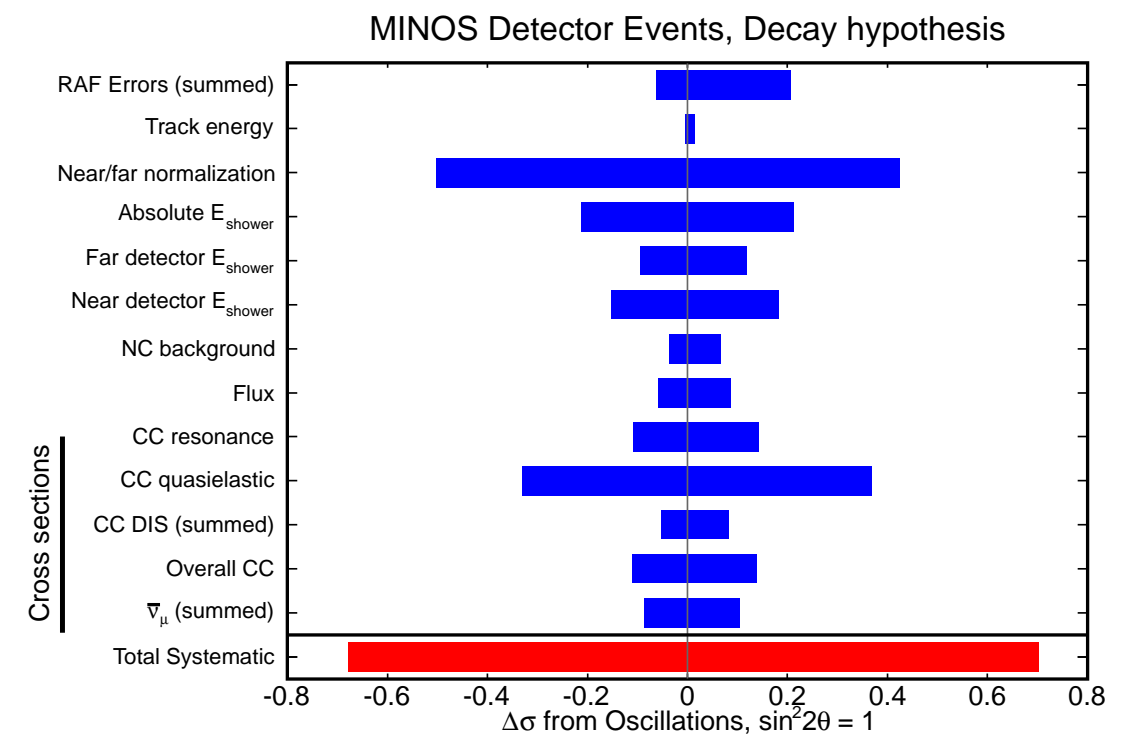

(a) Systematic error effect on the decay fit.

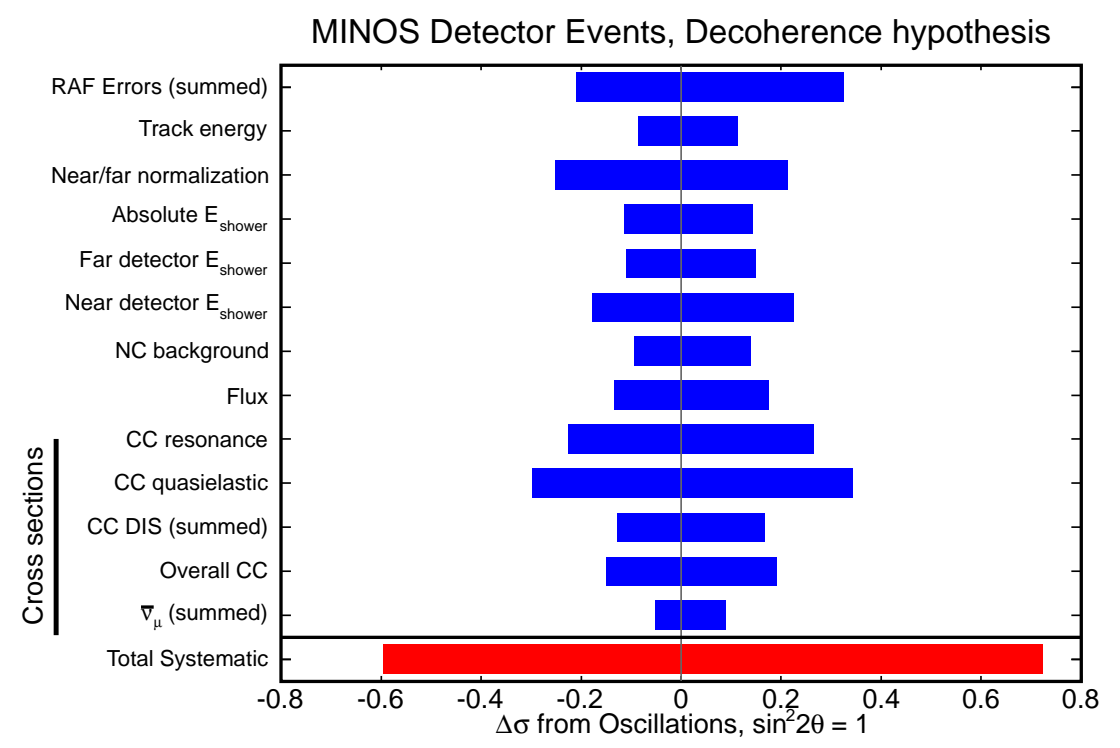

(b) Systematic error effect on the decoherence fit.

Figure 8.2: The effect of the systematic uncertainties on the sensitivity to (a) decay and (b)decoherence. This was modeled with high statistics fake data fiducial and rock and anti-fiducial samples[91]. 
fore, the fitting method must be modified from the general fitting procedure described in Chapter 2. The expected distribution at the Far Detector can be derived for each resolution bin. Then the log-likelihood function taking into account the number of resolution bins and $\bar{\nu}_{\mu}$ events is given by

$$
\chi^{2}=-2 \ln (L)=2 \sum_{i}^{N_{\text {tot }}} \sum_{j}^{N_{\text {res }}+P Q}\left(N_{i j}^{M C}-N_{i j}^{\text {Data }}+N_{i j}^{\text {Data }} \ln \left(\frac{N_{i j}^{\text {Data }}}{N_{i j}^{M C}}\right)\right)+\sum_{k}^{N^{\text {syst }}} \frac{a_{k}^{2}}{2 \sigma_{k}^{2}}
$$

which is quite similar to the general fitting equation given in equation (2.6). An alternative shape only fit was also considered and is described in detail by Stephen Coleman [58]. The partially reconstructed RAF sample can be fitted separately or simultaneously using a similar equation.

Two separate fitting frameworks are used to independently fit for the oscillation result. The NuSystFitter algorithm was used in the previous MINOS $\nu_{\mu}$ disappearance analyses. This algorithm uses the ROOT based MINUIT function to find the optimal value of the oscillation parameters and return a $\chi^{2}$ surface. The other fitting algorithm is called the GhostFitter. The GhostFitter is a new stand-alone package which uses a template system to handle nuisance parameters in the fit. This method reduces discontinuities in the $\chi^{2}$ derivative [58, 92].

\subsection{Far Detector Data Distributions and Statistics}

The neutrino oscillation parameters $\Delta m_{32}^{2}$, and $\sin ^{2}\left(2 \theta_{32}\right)$ are measured only after the Far Detector data distributions are examined for any unexpected variable-dependent deficits which would indicate a problem in the Far Detector data unrelated to oscillations. For example, in Figure 8.3, the position of the events in the Far Detector $\nu_{\mu}$ charged current sample is shown. Although an 
overall deficit in events is seen, the events are evenly distributed in all directions. This is expected for a neutrino deficit which is unrelated to the physical properties of the detector. Other distributions shown similar indications of no detector effects.

The Far Detector data unblinding was continued by comparing the number of events seen to the number expected without considering the oscillation hypothesis. In Table 8.2, the number of events which pass a series of selection criteria is given in each row for specific runs. The selections used in the current analysis are shown in bold red. This is generally the charged current/neutral current selection without removing positive curvature events. However, for the Run I pHE data set, positive curvature events are not used. This is because positive curvature events at high energy are considered a background and likely do not contain low-energy $\nu_{\mu}$ events with a misidentified charge-sign. The new charged current selection increases the number of events in the data-set used from 1926 to the sum of all the values in bold which is 1986 events. This is a statistical increase of 60 events (about 3\%). The 'Has a track', 'track has good fit' and 'good track data' selections select events with good tracks. The fiducial selection selects events whose vertex is within the fiducial volume described in Chapter 3. 'Data quality checks' ensure that the event is from a good data run.

In Figure 8.4 and Figure 8.5, the charged current/neutral current separation parameters, are shown for the Far Detector fiducial events before the charged current selection. In both distributions there is a deficit which has some correlation with the value of the variable. This is reasonable because the neutral current background is not affected by oscillations and high-energy $\nu_{\mu}$ charged current events are likely to have high values of the separation param- 


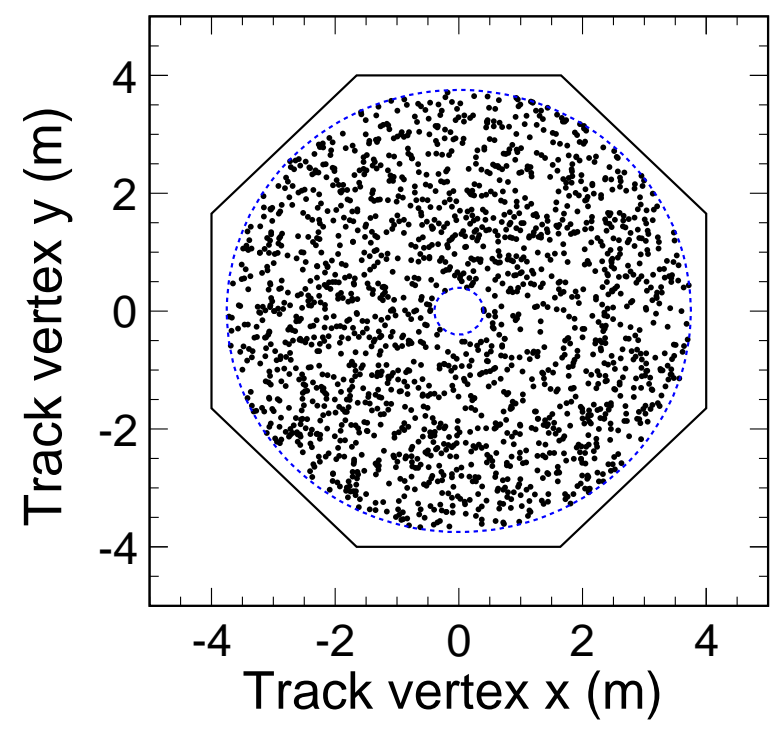

(a) X-Y vertex of tracks in the Far Detector

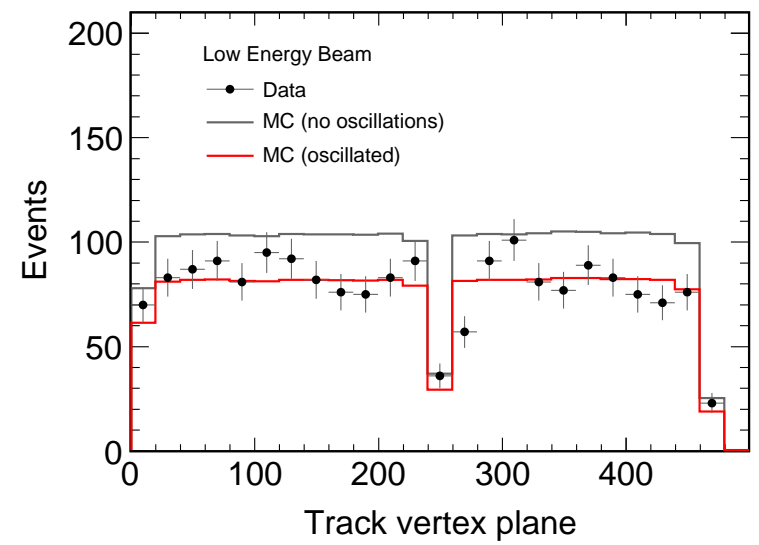

(b) Z vertex of tracks in the Far Detector

Figure 8.3: The position of events in the Far Detector. Left: The X and Y position of tracks in events classified as fiducial $\nu_{\mu}$ charged current events. Right: The $\mathrm{Z}$ vertex position of tracks in the Far Detector in events classified as fiducial $\nu_{\mu}$ charged current events. The red histogram is the expected distribution at the Far Detector given the Near Detector data, the black points is the observed energy spectrum of fully contained events, and the blue histogram represents the expected number of events given the best fit parameters. 


\begin{tabular}{lcccc|c}
\hline \multicolumn{5}{c}{ Events in the Far Detector by Run Number after different selections } \\
\hline \hline & Run I & Run I pHE & Run II & Run III & Total Data \\
\hline Raw Events & 8846 & 1616 & 8911 & 13622 & 32994 \\
Has a track & 891 & 331 & 1401 & 2604 & 5227 \\
Track has good fit & 886 & 330 & 1397 & 2595 & 5208 \\
good track data & 886 & 330 & 1397 & 2595 & 5208 \\
Fiducial & 433 & 178 & 694 & 1382 & 2688 \\
data quality checks & 433 & 172 & 665 & 1354 & 2624 \\
Total Preselection & 415 & 171 & 665 & 1306 & 2557 \\
\hline CC/NC Selection (2010) & 318 & 129 & 511 & $\mathbf{1 0 3 7}$ & 1995 \\
CC/NC Selection (2008) & 313 & 123 & 494 & 1003 & 1933 \\
$Q / P<0$ (2010 Selection) & 293 & $\mathbf{1 2 0}$ & 459 & 902 & 1774 \\
$Q / P<0$ (2008 Selection) & 289 & 116 & 448 & 877 & 1730 \\
\hline
\end{tabular}

Table 8.2: A catalog of the number of events in the Far Detector after different selections. The selection at each row incorporates all previous selections until the 'Total Preselection', which is the all selections other than the selection of events with values of the $\mathrm{CC} / \mathrm{NC}$ separation parameters greater than the 2010 thresholds. Afterward, the selection is explored for both the 2010 and 2008 CC selections and with and without the removal of positive curvature $(Q / P>=0)$ events. The values in bold are the selections used in the latest analysis and published in PRL [21]. 
eters. Therefore, at high values, high-energy events dominate. These events experience less of an effect due to oscillations.

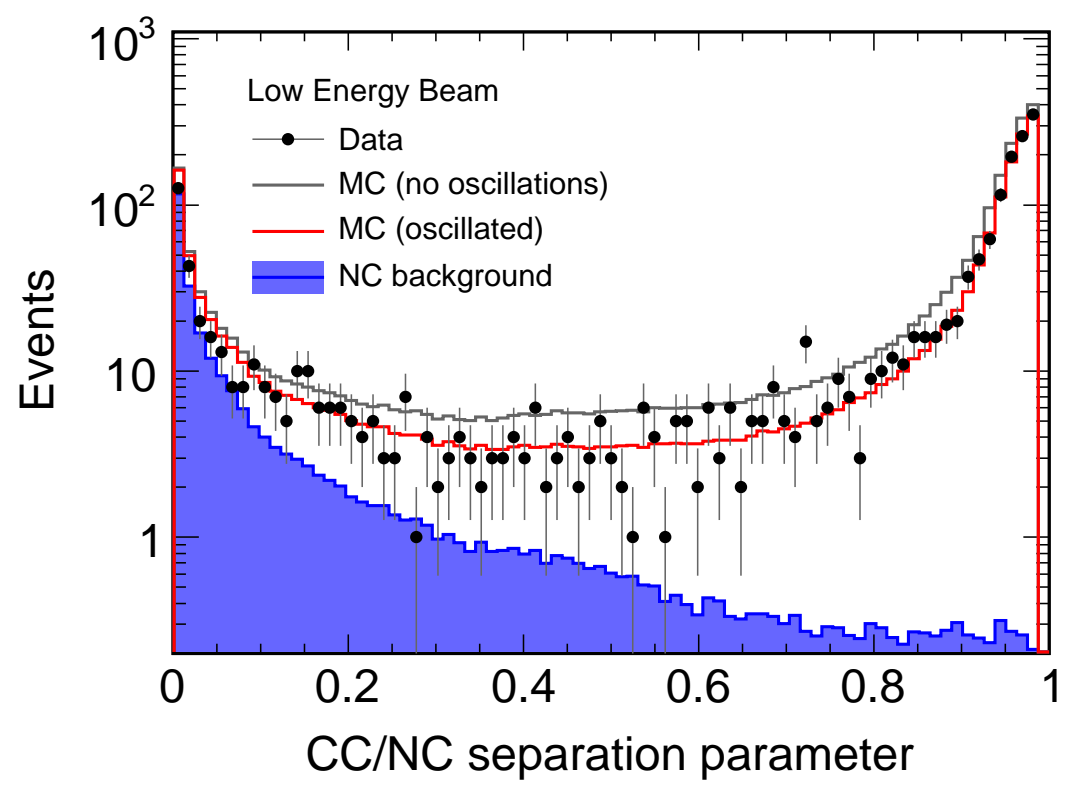

Figure 8.4: The first charged current to neutral current separation parameter ( $\mu \mathrm{ID}-\mathrm{A})$ for the Far Detector. The red histogram is the expected distribution at the Far Detector given the Near Detector data, the black points is the observed energy spectrum of fully contained events, and the blue histogram represents the expected number of events given the best fit parameters.

The ratio of the number of in the selection defined by the thresholds in equation (6.2) (used in the latest analysis [21]) to the number of events in the selection defined by the thresholds of equation (6.1) (used in the 2008 analysis [33]) is shown for the different resolution bins and versus the neutrino energy in Figure 8.6. Events are gained in all bins, signifying that this method increases the acceptance of high-resolution quasi-elastic like low energy neutrino events. The table in Figure 8.6 has the relevant number of events in each bin and the 


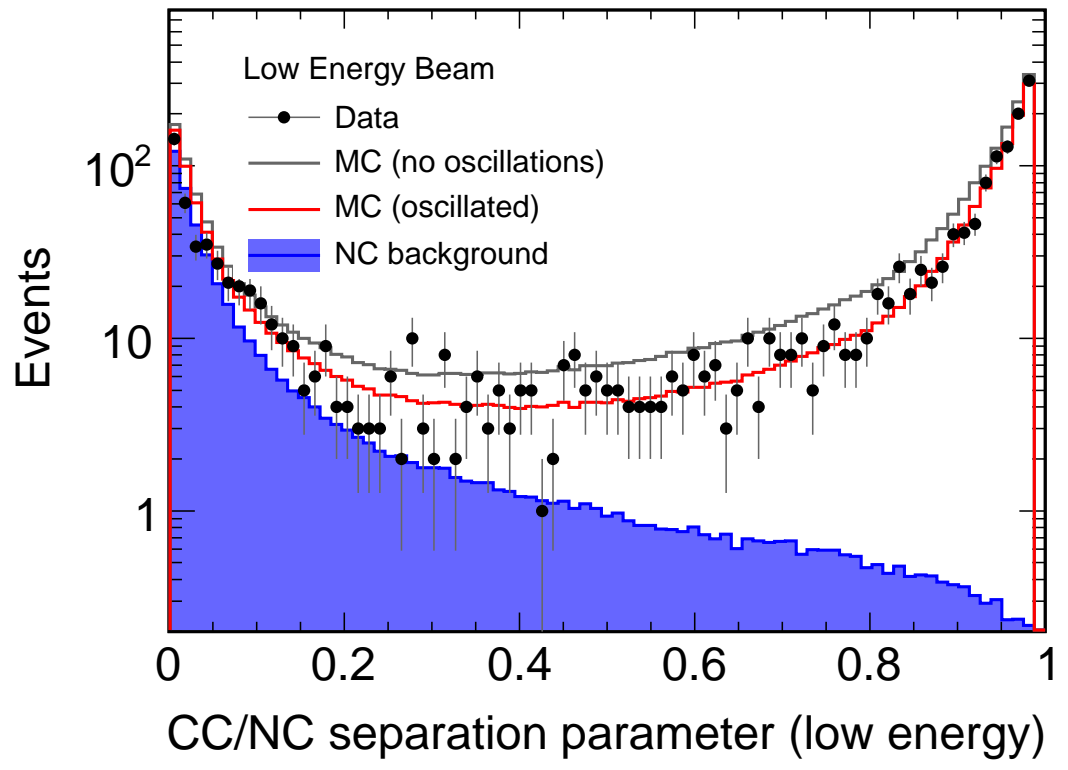

Figure 8.5: The second low energy charged current to neutral current separation parameter ( $\mu \mathrm{ID}-\mathrm{B})$ for the Far Detector. The development of this parameter was discussed in Chapter 4 . The red histogram is the expected distribution at the Far Detector given the Near Detector data, the black points is the observed energy spectrum of fully contained events, and the blue histogram represents the expected number of events given the best fit parameters. 
number of events gained by the new threshold values. The events gained by the relaxing the thresholds from equation (6.1) to equation (6.2) is shown in Table 8.3.

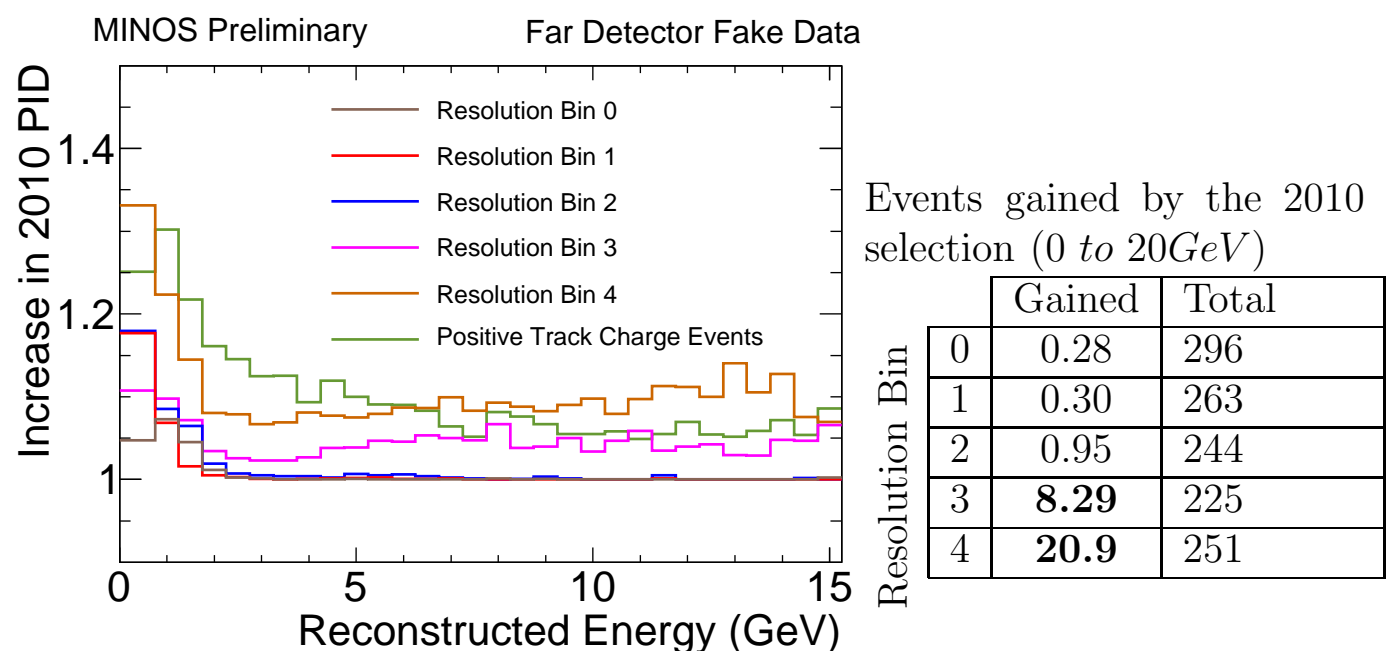

Figure 8.6: Events gained in Resolution Bins: For the new PID the change (ratio of New/Old PID) in energy spectra is plotted for each of the resolution bins. Note that in the final analysis the resolution bins will be redefined such that the number of events in each resolution quintile is the same. Left: a plot of the increase due to the new selection for each resolution bin versus energy. Right: The total increase given in both the number of events increase and the total number of events in each bin. The values made bold for resolution bins 3, 4, and the positive curvature $(Q / P>0)$ emphasize the resolution bins that see the largest increase in expected events. The increase in positive $\mathrm{Q} / \mathrm{P}$ values is expected since at low energies, the resolution of $\mathrm{Q} / \mathrm{P}$ is low.

Eight out of thirty-eight events in the Run I through Run III data set selection are gained below $2 \mathrm{GeV}$. Recall that this low-energy region is where one expects to see the largest deficit of $\nu_{\mu}$ events due to oscillations. Therefore, assuming a neutrino deficit at low energies due to oscillations, the number of events gained at low energy should be less than expected under the assumption of no oscillations for the same relaxed thresholds. The new thresholds also 


\begin{tabular}{|l|l|l|l|}
\hline Run Number & 2010 Selection & 2008 Selection & Increase \\
\hline \multicolumn{4}{|c|}{ All Q/P values } \\
\hline Run I & 10 & 9 & $1(11 \%)$ \\
Run II & 12 & 8 & $4(50 \%)$ \\
Run III & 16 & 13 & $3(1.23 \%)$ \\
\hline \multicolumn{4}{|c|}{ Negative Curvature $(Q / P<0)$} \\
\hline Run I & 7 & 6 & $1(16 \%)$ \\
Run II & 10 & 7 & $3(42 \%)$ \\
Run III & 10 & 10 & $0(0 \%)$ \\
\hline
\end{tabular}

Table 8.3: The latest analysis charged current selection (2010 selection [21]) compared to the selection previously used (in the 2008 analysis [33]). All events are shown in the first three rows. The bottom rows show the same, but for only negative charge-sign events.

increase the number of events expected to be seen in this same region and increases the statistical significance of the observed deficit. The majority of the events reclaimed in the low-energy region by the new charged current selection can be seen in event displays in Figure B. These event displays demonstrate that the events reclaimed by the new charged current selection are short-track events, which primarily have clean, straight tracks.

We can consider how these gained events are distributed with respect to the two $\mu \mathrm{ID}$ variables and energy. In Figure 8.7 and Figure 8.8, the events gained by the second $\mathrm{CC} / \mathrm{NC}$ discriminator $(\mu \mathrm{ID}-\mathrm{B})$ and the new thresholds are shown versus their value of the PID parameters. This demonstrates that the gained events are evenly distributed in values of $\mu \mathrm{ID}-\mathrm{A}$ and $\mu \mathrm{ID}-\mathrm{B}$ and primarily at low energy. Overall, 60 fiducial events were gained with the new thresholds in the $7.2 \times 10^{20} \mathrm{POT}$ analysis. 


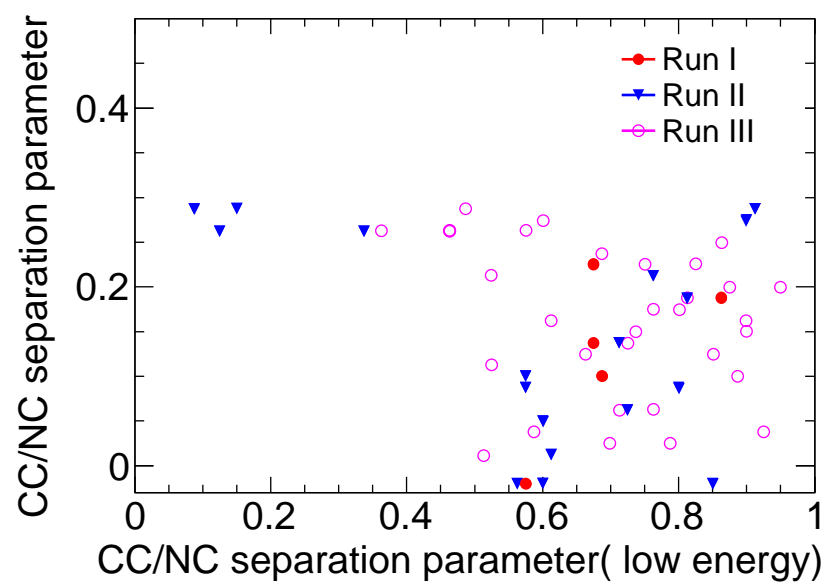

Figure 8.7: The events gained by the new analysis of the charged current selection. The events are separated by run number with red points representing Run I, blue triangles representing Run II, and magenta open points representing Run III. The $\mathrm{x}$-axis is $\mu \mathrm{ID}-\mathrm{B}$ or "CC/NC separation parameter(low energy). The $\mathrm{y}$-axis is $\mu \mathrm{ID}-\mathrm{A}$, developed by $\mathrm{R}$. Ospanov, and used in the previous analysis [33]. 


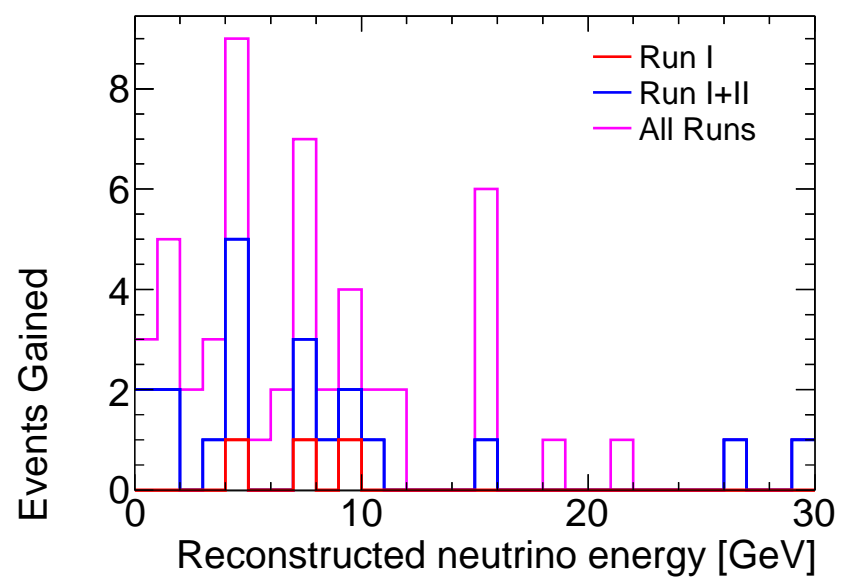

Figure 8.8: The reconstructed energy of these events is shown. The histograms are stacked such that the red histogram represents purely Run I, the blue histogram represents events in Run I and II, and the magenta histogram represents the events of all three runs. 


\subsection{Measurements of $\nu_{\mu}$ Disappearance in the Far De- tector}

The number of events observed and expected for each run is shown in Table 8.4 [21]. The number of expected events for each run differs due to differences in beam exposure and running conditions. A clear deficit of events is seen in every NuMI run. The fraction of the expected events observed ranges by percentage from near $75 \%$ for Run I (low energy) to close to $95 \%$ for the Run I pseudo-high-energy configuration. The partially reconstructed RAF events report a smaller deficit simply due to the energy distribution of these events (which peaks at higher energy). 1986 fully reconstructed fiducial events and 2017 partially reconstructed RAF events were observed in the Far Detector. The expected number of events at the Far Detector for the same exposure is 2561 fiducial and 2206 RAF events. This is a deficit of $81 \%$ and 91\% for fiducial and RAF events respectively.

\begin{tabular}{|l||l|l||l|l|}
\hline Run Number & \multicolumn{2}{|c|}{ Observed } & \multicolumn{2}{c|}{ Expected } \\
\hline & Fiducial & RAF & Fiducial & RAF \\
Run I & 318 & 357 & 426 & 375 \\
Run IpHE $(\mathrm{Q} / \mathrm{P}<0)$ & 120 & 128 & 134 & 136 \\
Run II & 511 & 555 & 639 & 565 \\
Run III & 1037 & 977 & 1252 & 1130 \\
\hline Total & 1986 & 2017 & 2451 & 2206 \\
\hline \hline
\end{tabular}

Table 8.4: The number of events observed and expected for each NuMI run including both partially reconstructed RAF events and fully reconstructed fiducial events.

A reanalysis of the Run I and Run II data (without the data accumulated from the last result) was performed as part of the latest 2010 analysis 
[94]. This reanalysis used only statistical errors and began with 2008-like analysis, including using the 2008 thresholds given in equation (6.1). The purpose of this reanalysis was to determine the effect of each analysis improvement on the oscillation result. The results of which are summarized in Table 8.5. Using the new selection given in equation (6.2) and the k-NN shower energy estimate reduces the value of $\sin ^{2}\left(2 \theta_{32}\right)$, bringing it out of the unphysical region of $\sin ^{2}\left(2 \theta_{32}\right)>1$. The values given with a "2010 analysis on the 2008 event sample" is consistent with the result published in 2008 [33, 94].

\begin{tabular}{l|c|c}
\hline \multicolumn{3}{c}{ Statistics-Only Reanalysis of Runs I and II (with pHE) } \\
\hline $\begin{array}{l}\text { 2008 Analysis with dogwood Re- } \\
\text { construction }\end{array}$ & $\begin{array}{c}\Delta m_{32}^{2} / 10^{-3} \mathrm{eV}^{2} \\
2.22\end{array}$ & $\begin{array}{c}\sin ^{2}\left(2 \theta_{32}\right) \\
1.06\end{array}$ \\
\hline $\begin{array}{l}\text { With new separation parameter } \\
\text { only }\end{array}$ & 2.36 & 0.99 \\
\hline $\begin{array}{l}\text { With new kNN shower energy es- } \\
\text { timate only }\end{array}$ & 2.29 & 0.97 \\
\hline $\begin{array}{l}\text { With both new separation param- } \\
\text { eter and energy estimate }\end{array}$ & 2.43 & 0.91 \\
\hline
\end{tabular}

Table 8.5: Statsistics-only fits of Run I and Run II fiducial data. This table shows the effect of the new k-NN shower energy estimate and separation parameter thresholds have on the previous result.

The Far Detector data is binned in five separate resolution bins and a sixth positive curvature $(Q / P>0)$ group. Each resolution bin should have roughly the same number of Far Detector events, with or without oscillations. The number of events in each bin is shown in Table 8.6. The energy spectrum of each bin is shown in Figure 8.9. The disappearance in each bin is the same.

The energy spectrum for the events observed and cataloged in Table 8.4 


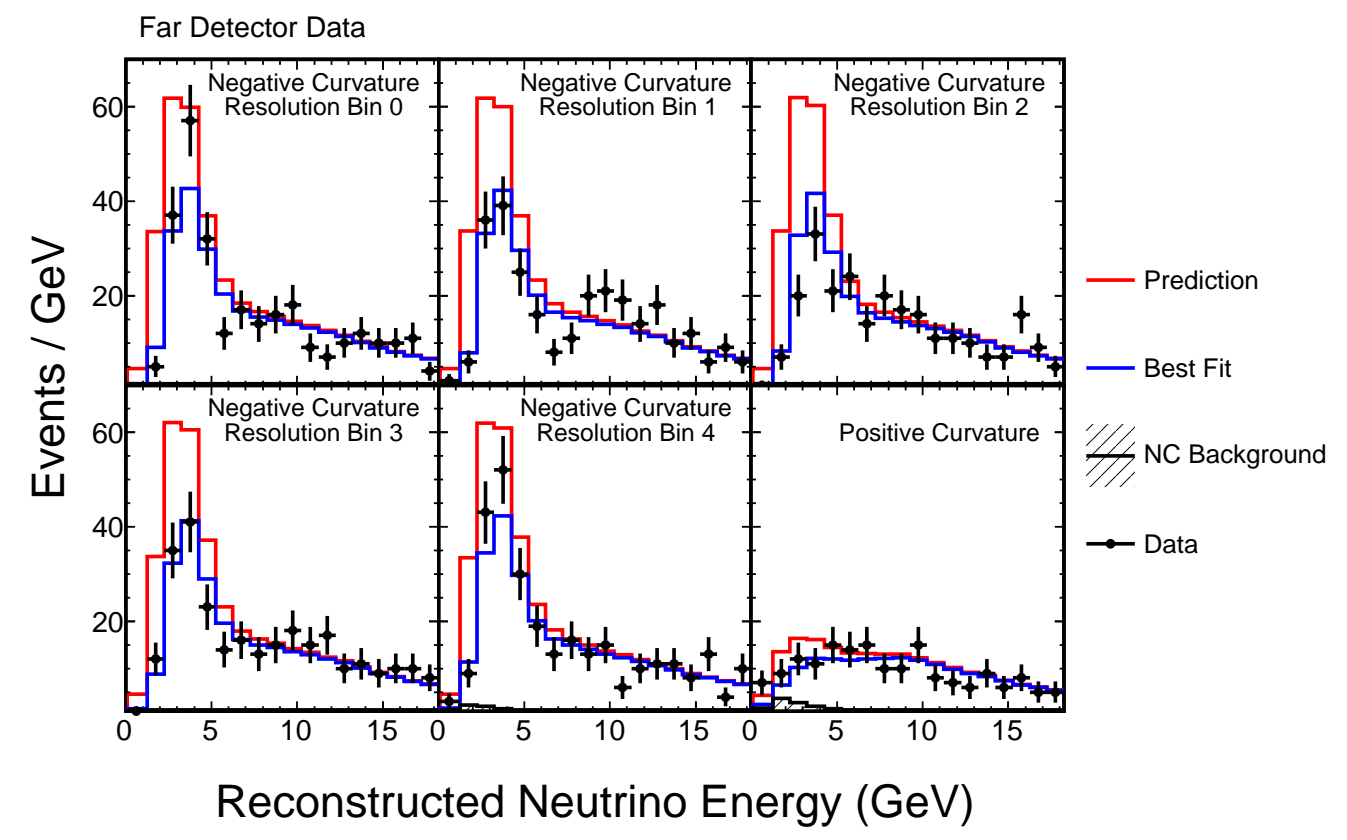

Figure 8.9: The Far Detector fully contained event energy spectrum divided into five resolution 'bins' and a positive curvature bin. The red histogram is the expected distribution at the Far Detector given the Near Detector data, the black points is the observed energy spectrum of fully contained events, and the blue histogram represents the expected number of events given the best fit parameters. 


\begin{tabular}{|l|cccc|c|}
\hline Resolution Bin & Run I LE & Run I pHE & RunII & Run III & All Runs \\
\hline Bin 0 & 63 & 25 & 106 & 168 & 362 \\
Bin 1 & 52 & 28 & 85 & 193 & 357 \\
Bin 2 & 59 & 25 & 87 & 150 & 321 \\
Bin 3 & 60 & 16 & 89 & 186 & 351 \\
Bin 4 & 59 & 26 & 93 & 205 & 383 \\
Q/P $>0$ & 25 & - & 52 & 135 & 212 \\
\hline Total 2010 Selection & 318 & 120 & 511 & 1037 & 1986 \\
\hline \hline
\end{tabular}

Table 8.6: The Far Detector fully reconstructed events separated into the five resolution bins and sixth positive curvature bins. Such events have passed all the standard selections, including the $\mathrm{CC} / \mathrm{NC}$ selectors.

are shown in Figure 8.10 for fully reconstructed fiducial events and Figure 8.11 for partially reconstructed RAF events. The data at the Far Detector is compared to the predicted spectrum given no oscillations and the prediction given the oscillation parameters from the best-fit of the data. On each plot, the ratio to the no oscillation prediction is given underneath the energy spectrum. In Figure 8.10 this ratio is compared to the best-fits for decay, decoherence and oscillations and in Figure 8.11, this ratio is compared to just the oscillation hypothesis. RAF events suffer from poor energy resolution, which is why the energy spectrum in Figure 8.11 cannot resolve the low-energy dip.

For all runs combined including positive curvature and the partially reconstructed RAF events, the oscillation parameters were found to be

$$
\begin{array}{rc}
\sin ^{2}\left(2 \theta_{32}\right) & =1_{0.29} \\
\Delta m_{32}{ }^{2} & =2.16_{0.18}^{+0.62} \times 10^{3} \mathrm{eV}^{2}
\end{array}
$$

$\sin ^{2}\left(2 \theta_{32}\right)$ was found to be greater than 0.90 at a confidence of $90 \%$ and greater than 0.94 at a confidence of $68 \%$. The best fit value of $\sin ^{2}\left(2 \theta_{32}\right)$ is at 1.00 . 


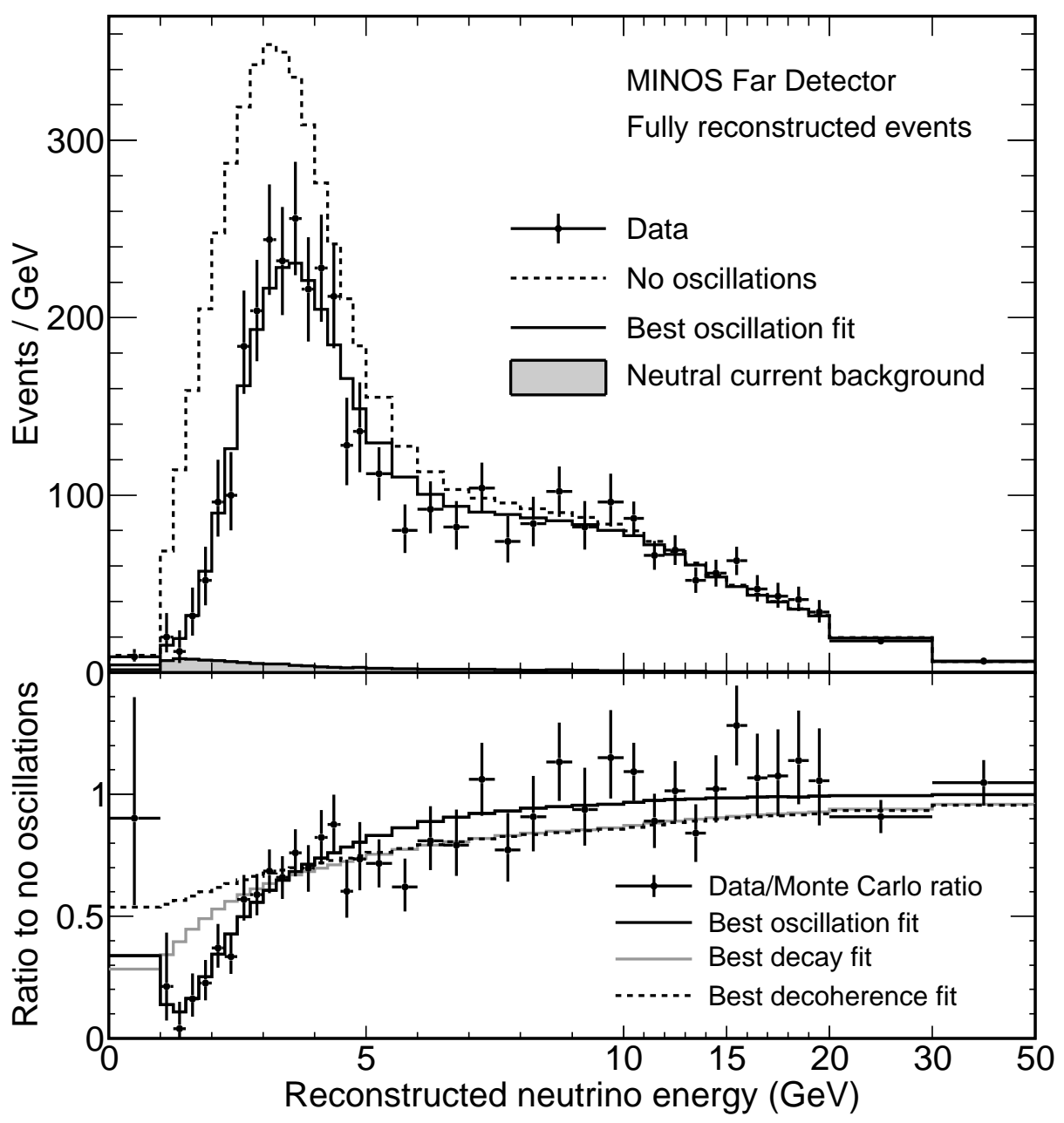

Figure 8.10: Top: The energy spectrum of fully reconstructed events in the Far Detector which were classified as $\nu_{\mu}$ charged current interactions. The dashed histogram represents the spectrum predicted from the Near Detector measurements assuming no oscillations, while the solid histogram reflects the same assuming the best fit oscillation parameters ( from the fit to all seven event categories). Bottom: The points with error bars are the background-subtracted ratios of data and the best oscillation hypothesis to the no-oscillation hypothesis. Lines show the best fits for the three tested scenarios: oscillations, decay and decoherence. 


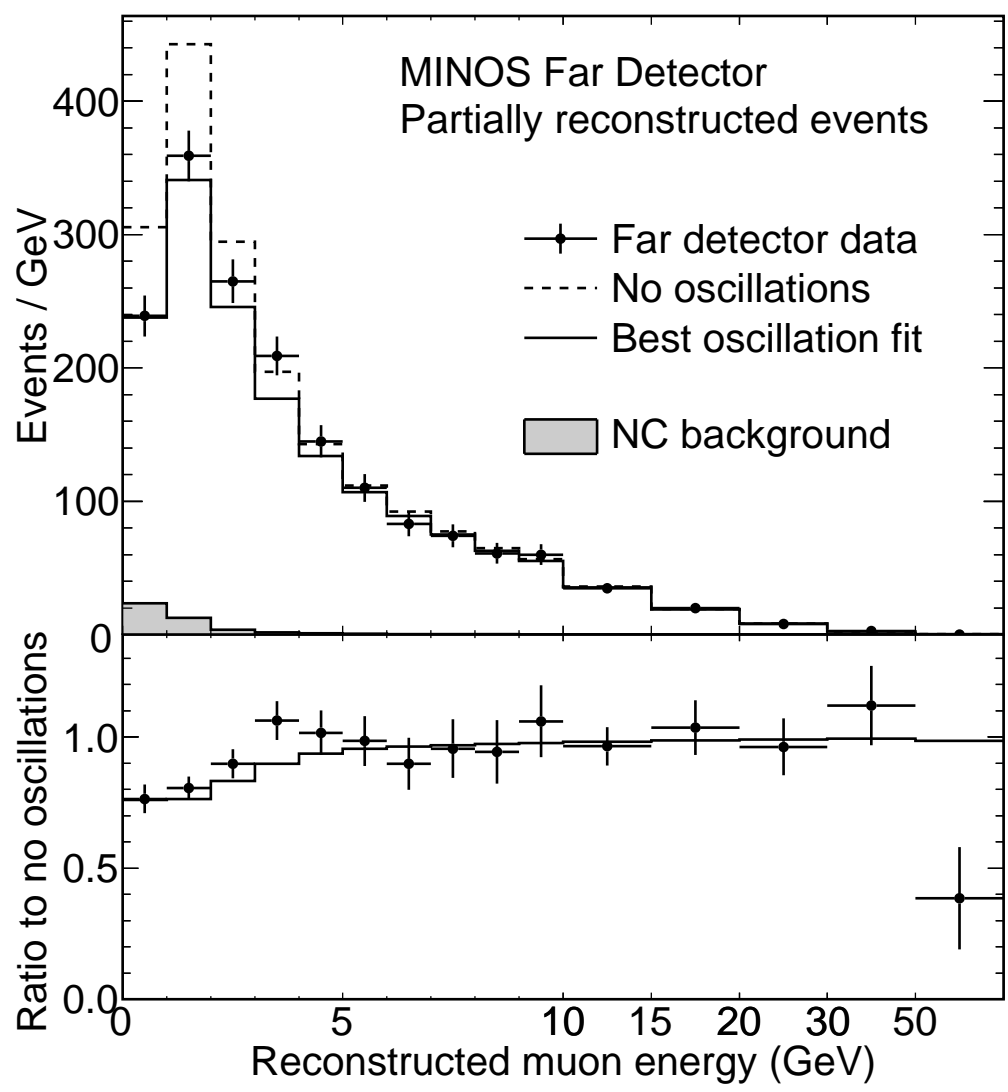

Figure 8.11: Top:The Far Detector energy spectrum for partially reconstructed charged current events (RAF). The dashed histogram represents the spectrum of partially contained events predicted by the Near Detector measurements assuming no oscillations, while the solid histogram reflects the same assuming the best fit oscillation parameters ( form the fit to all seven event categories). Bottom: The points with error bars are the background-subtracted ratios of data and the best oscillation hypothesis to the no-oscillation hypothesis. 
Unfortunately, this analysis has very poor sensitivity to $\sin ^{2}\left(2 \theta_{32}\right)$ compared to other published results. The most accurate measurement coming from the Super-Kamiokande experiment with $\sin ^{2}\left(2 \theta_{32}\right)>0.965$ at $90 \%$ confidence[21]. The pure decay and decoherence models were disfavored at the 7 and 9 standard devation level respectively, using the definition previously stated in this chapter.

The confidence contours and best-fit values for $\Delta m_{32}^{2}$ and $\sin ^{2}\left(2 \theta_{32}\right)$ are shown in Figure 8.12. This is a significant measurement for the MINOS experiment; it produced the world's best measurement of $\Delta m_{32}^{2}$. Furthermore, this is likely the last measurement of the oscillation parameters by MINOS since the errors on these parameters are now limited by systematic rather than statistical errors. 


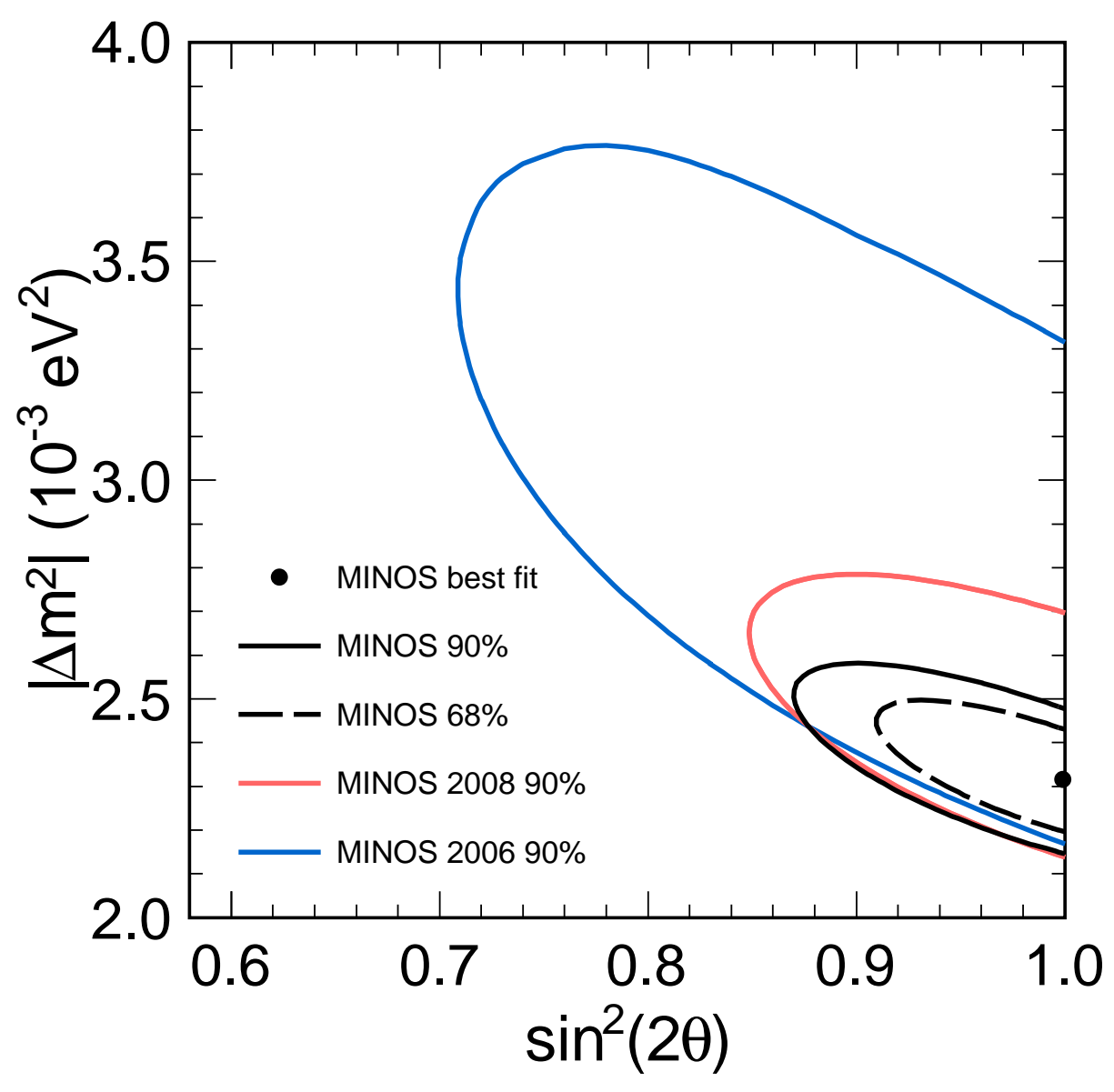

Figure 8.12: Contour results using fiducial and RAF events. The previous MINOS results for the $90 \%$ confidence intervals are shown in blue (2006) and red (2008) [33] respectively. The $68 \%$ and $90 \%$ confidence intervals for the latest (2010) measurement is shown in black dashed and solid lines. The best fit point, which occurs at maximal mixing, is shown as a black point. 


\section{Chapter 9}

\section{Charged Current $\nu_{\mu}$ Identification in Minerva}

Chapter 4 described improvements to the identification of low-energy muons for MINOS. Although the MINOS experiment continues to study improvements to muon identification through different data-mining techniques [95], in the future the NuMI beam will be configured to focus higher-energy neutrinos. At that time, the MINOS detectors will be used as part of the MINOS + experiment whose goal is searching for non-standard neutrino physics at high energy [96]. Therefore, increasing the efficiency of low-energy muon identification is no longer a concern for MINOS measurements.

The MINER $\nu \mathrm{A}$ experiment will be exposed to the future high-energy NuMI beam as well. However, MINER $\nu$ A's measurement of low energy neutrino cross-section measurements is more significantly affected by the charged current selection at low energy and MINER $\nu \mathrm{A}$ is currently analyzing data from the LE configuration running of the NuMI beam. MINER $\nu \mathrm{A}$ originally had no muon identification scheme in place beyond identifying tracks which enter the MINOS Near Detector as high energy muons. Therefore, MINER $\nu \mathrm{A}$ benefits from a muon identification algorithm similar to the ones in place for the MINOS experiment. In contrast with the MINOS, whose detectors were designed to reconstruct high-energy muons, the MINER $\nu$ A detector was designed to identify low-energy $(0.1-2 \mathrm{GeV})$ hadronic particles and high energy muons. This presents different challenges to low-energy muon identification 
and charged current selection than those faced by the MINOS experiment. In this chapter, we continue our investigation of muon identification at low-energy by focusing on muon identification for the MINER $\nu \mathrm{A}$ experiment.

\subsection{Hadronic Particle Identification in MINER $\nu \mathrm{A}$}

The physics goals of MINER $\nu \mathrm{A}$ include measuring exclusive and inclusive neutrino cross-sections. It is therefore imperative for MINER $\nu \mathrm{A}$ to identify not only muons, but also the pions and protons that are produced in specific final states. For these reasons, MINER $\nu \mathrm{A}$ was designed with smallerwidth scintillator strips and scintillator composes a much larger fraction of the MINER $\nu \mathrm{A}$ detector than in the MINOS detectors (as discussed in Chapter 3). The large fraction of low-density scintillator in the MINER $\nu \mathrm{A}$ detector allows hadronic particles to travel further than in the MINOS detector. Thus, hadronic particle tracks are easier to reconstruct. In addition, the finely grained scintillator allows $\mathrm{MINER} \nu \mathrm{A}$ to resolve the ionization profiles of hadronic particles. Because of the steel in the detector, MINOS is unable to perform such refined measurements.

In order to identify the various hadronic particles, the MINER $\nu \mathrm{A}$ experiment developed particle identification tool which utilizes the Bethe-Bloch ionization profile. This tool fits the ionization profile of a track with the average ionization curve for pions, kaons, and protons. The ionization profile for each particle differs because of the different masses of these particles. A visual example of using the energy loss profile as a particle identification tool can be seen in Figure 3.1. Most of the separation between the ionization profiles is seen at low-energies. This is equivalent to stating that most of the separation between the ionization profiles is at the end of the particle track, where the 
particle is nearing the end of its life before capture or decay.

In Figure 9.1, The average energy loss per centimeter on a track between 2 to 7 centimeters from the end of the track is shown for pions and protons. The large difference in the average energy loss between pions and protons is indicative of the differences in their ionization profile. The ionization profile particle identification technique (IPPID) is being actively developed. The IPPID returns a best-particle hypothesis (for example proton) with a best-fit energy for this ionization profile. For example, a MINER $\nu$ A'prong', which is a collection of MINER $\nu \mathrm{A}$ reconstructed objects, could be found to have the best-fit ionization profile of a $250 \mathrm{MeV}$ pion. This result would be quoted with an error on the energy of the 'pion' and a $\chi^{2}$ value, demonstrating the goodness-of-fit of the ionization hypothesis.

The IPPID shows great promise at separating pions, kaons, protons, and other hadronic particles from each other. However, because of the similar mass of the pion and muon $\left(135 \mathrm{MeV} / \mathrm{c}^{2}\right.$ for the pion versus $105 \mathrm{MeV} / \mathrm{c}^{2}$ for the muon) the ionization profile for pions and muons is too similar to be used as the sole distinguishing characteristic. Once again, like for the muon identification in MINOS discussed in Chapter 4, we can use the nuclear interactions versus minimum ionization signatures to separate pions from muons more directly.

\subsection{Muon Identification using a MINOS k-NN approach}

The method used by MINOS and applied to MINER $\nu$ A may help separate pions and muons for a variety of reasons. When fitting the MINER $\nu \mathrm{A}$ tracks with an ionization profile, the IPPID attempts to ignore non-ionization behavior in order to estimate the energy through the ionization profile. Instead, the IPPID creates categories which indicate if there was non-ionizing 


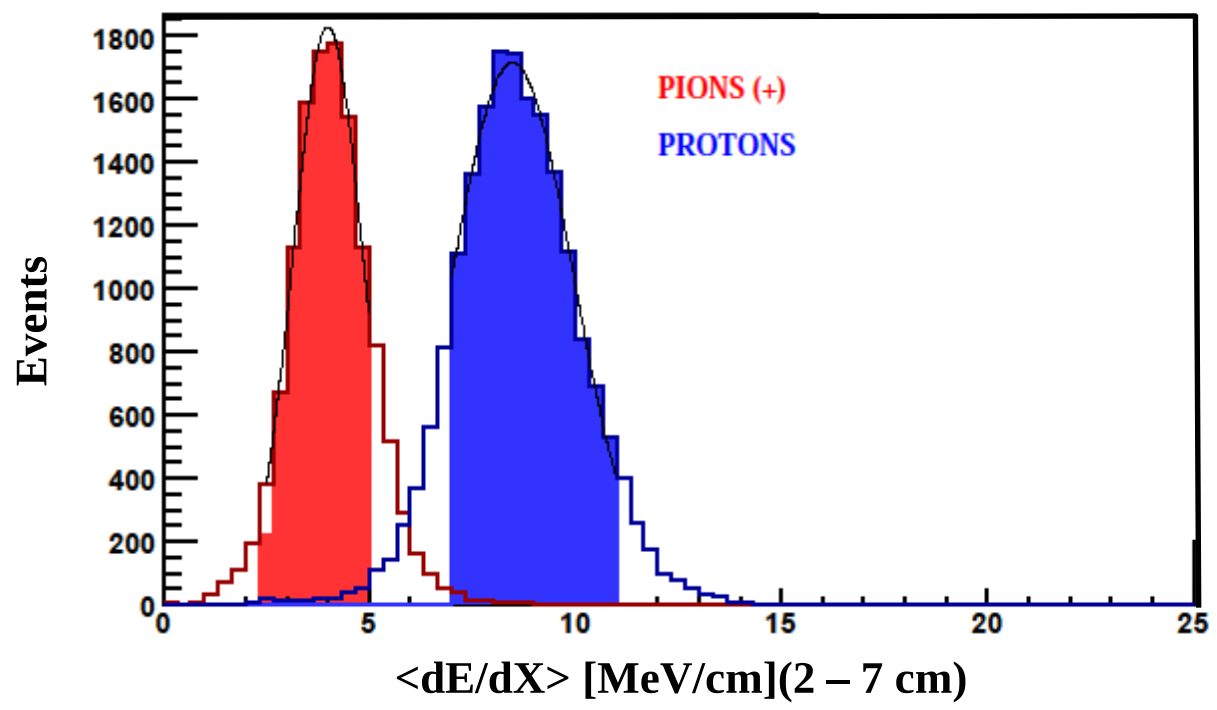

Figure 9.1: The average value of the energy lost per $\mathrm{cm} 2$ to $7 \mathrm{~cm}$ from the end of the track for pions and protons.Protons have momentum between 0.45 $\mathrm{GeV}$ and $1.25 \mathrm{GeV}$. Pions have momentum between $0.15 \mathrm{GeV}$ and $0.60 \mathrm{GeV}$. All particles are well-contained in MINER $\nu$ A's Inner Detector region. This plot is adapted from a MINER $\nu \mathrm{A}$ presentation by Tammy Walton[97]. 
behavior. This single variable does not have the variability separation of a well-thought PID. In contrast to the IPPID's multi-tasking nature, a k-NN algorithm is only concerned with particle identification. Because of it's limited responsibilities, the $\mathrm{k}-\mathrm{NN}$ algorithm can directly use the amount of nonionizing behavior to distinguish pions from muons. ${ }^{1}$

\subsubsection{Categories of Particles in MINER $\nu \mathrm{A}$}

The MINER $\nu \mathrm{A}$ experiment initially used a very strict definition for muon identification; only prongs with tracks matched to energy depositions in the MINOS Near Detector were classified as muons. This energy deposition in the MINOS Near Detector could take the form of a few hits or an entire track. An example of such a 'Minos-Matched' prong was shown in Figure 3.16. Prongs with a MINER $\nu \mathrm{A}$ track that is matched to non-tracked energy depositions in MINOS are called 'Minos-Stub' prongs. Minos-Matched and MinosStub prongs are necessarily high energy, minimum ionizing, and small zenith angle particles. Thus, these categories are overwhelmingly high energy, small zenith angle muons. Therefore, this categorical selection prefers muons with high energy and small angle, rather than selecting all kinematics with equal efficiency. Therefore, the selection of charged current muon neutrino events is 'sculpted' towards high energy as well.

Such sculpting of the event selection is a serious problem for MINER $\nu \mathrm{A}$. Consider the effect of such a selection simulated with Monte Carlo. If the muon kinematics of the Monte Carlo are incorrectly modeled, the measurement of

\footnotetext{
${ }^{1}$ It has been suggested that if MINER $\nu \mathrm{A}$ can identify delta rays, this might be another way that MINER $\nu \mathrm{A}$ can identify low-energy muons, independent of the $\mathrm{k}-\mathrm{NN}$. This is a signature that could be easily incorporated into the IPPID.
} 
the cross-sections would be incorrect. Therefore, large systematic uncertainties arise. MINER $\nu \mathrm{A}$ is especially interested in the kinematic dependence of the cross-section measurements. This is much harder to resolve with the reduced and sculpted selection of charged current muon neutrino events. Therefore, MINER $\nu \mathrm{A}$ needs a muon selection that is not biased towards a specific kinematic region, and must find a way to include charged current events that do not meet the 'muon-interacts-with-minos' requirement.

However, interaction with the MINOS Near Detector is still the best way to identify high-energy muons. Therefore, the MINOS-MINER $\nu \mathrm{A}$ track matching algorithm was improved to consider all tracks with trajectories that leave MINER $\nu \mathrm{A}$ as possible candidates for a match to MINOS. Another algorithm was developed to match tracks to Outer Detector hits in order to gain mor information. Unlike the Inner Detector, the Outer Detector scintillator strips in all modules are parallel to one of the hexagonal sides of the MINER $\nu \mathrm{A}$ detector. Such a geometry can only give 2-dimensional information about the trajectory of a particle. Therefore, tracks cannot be reconstructed in the MINER $\nu$ A Outer Detector. However, tracks which originate from MINER $\nu$ A's Inner Detector region can be projected into the Outer Detector. Clusters (A cluster is a group of scintillator 'hits', or energy deposits) near a projection of a track would indicate that the particle which made the Inner Detector track continued to travel through the Outer Detector. This projection method allowed MINER $\nu \mathrm{A}$ to for "ODMatch" prongs, which contained a track (or series of connected track segments) and the Outer Detector hits which are associated with that track.

An example of an ODMatch prong is seen in Figure 3.17, where a track (line) is seen projected to the Outer Detector, where a the track continues. If 
two tracks point towards the same Outer Detector energy deposit, the energy is associated with the nearest track. Clusters associated with clusters now associated with the track are also included into the final prong. Thus, a large hadronic shower on a line of a track projection into the detector would be associated with that track. Muons, which are minimum ionizing are expected to have associated clusters that are near the track projection.

The definitions of the five final prong categories are specified exactly below: ${ }^{2}$

- Minos-Matched: These prongs are matched to a track in the MINOS Near Detector. There are three categories of MINOS matching: Gold, Silver, and Bronze. These categories suggest how well the track is matched to MINOS.

- Minos-Stub: These prongs are matched to non-tracked energy deposition in the first six planes of the MINOS Near Detector.

- Contained: These prongs have a one or a series of track segments whose end is not well-contained by the 0.85 meter apothem hexagon that defines the radial fiducial volume or whose last track segment ends in the last four planes of the HCAL. Examples of events with contained prongs can be seen in Figure 3.18 and Figure 3.19 (neutral current and charged current DIS event respectively).

- ODMatched:ODMatch prongs are a series of track segments whose last

\footnotetext{
${ }^{2}$ After the work completed in this thesis, some of the definitions used by MINER $\nu \mathrm{A}$ were changed as the reconstruction was improved. Prongs now hold 'tags', which detail the exact parts of the detector the track traversed.
} 
position and direction vectors can be projected to be within $10 \mathrm{~cm}$ of a $0.2 \mathrm{MeV}$ or higher pulse-height cluster in the Outer Detector.

- Not Contained: These prongs contain the connected track segments that are not claimed by any of the preceding categories

In Figure 9.2, muons from a Monte Carlo simulation of neutrino interactions in the MINER $\nu \mathrm{A}$ detector with the LE010185N NuMI beam are divided into different categories. The histograms are stacked. The bottom histogram represents all "Minos-Matched" muons. Note that this is the majority of high-energy muons, but represents only a fraction of the muons at energies lower than $5 \mathrm{GeV}$. The histogram directly above this shows the small fraction of "Minos-Stub" muons. The stack of these two histograms is the spectrum of muons selected using a 'Matched-With-MINOS' muon selection. Note that this selection sculpts the selection of events towards high energy. Low energy neutrinos which produce low-energy muons in charged current reactions are selected with an extremely low efficiency. The remaining histograms in Figure 9.2 represent muons which are fully contained in the MINER $\nu \mathrm{A}$ detector (in green), "ODMatch" prongs (in yellow), or otherwise unspecified "Not-contained" prongs (in blue). Roughly 45 to 50 percent of muons below $3 \mathrm{GeV}$ are classified in these last three categories. In order to identify muons with such event topologies, we return to the k-NN method used by the MINOS experiment as discussed in Chapter 4. Because the IPPID can easily identify protons and kaons from pions and muons, we are most concerned with pion-muon separation.

In Figure 9.3, the average cluster energy in the Tracker region is shown, divided into the three non-MINOS related prong categories for muons and 


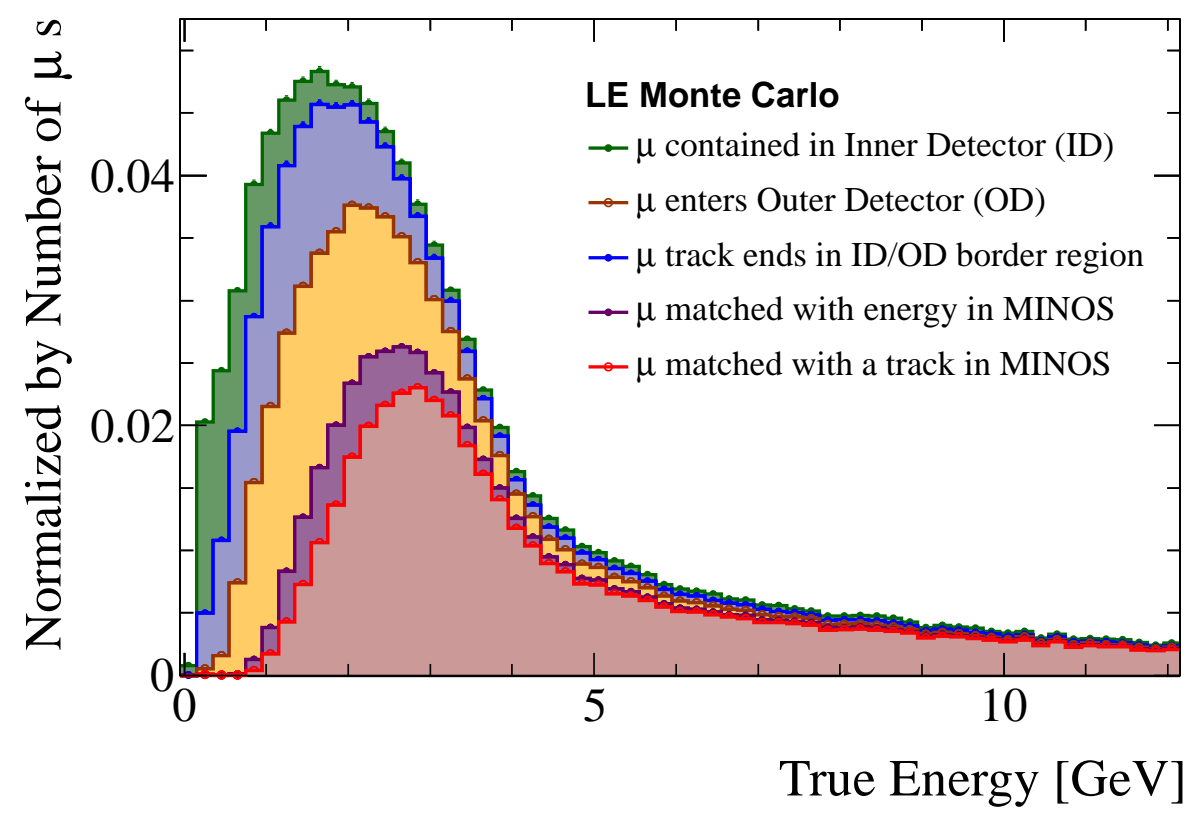

Figure 9.2: The muon energy spectrum of the MINER $\nu$ A experiment. The spectrum is split into different categories and the resulting histograms are stacked. Notice that less than $50 \%$ of muons with energy less than $5 \mathrm{GeV}$ enter the MINOS Near Detector and leave a reconstructed track. 
non-muons. Most muons are Not-Contained or ODMatched prongs. This is expected because such minimum ionizing muons are likely to have the long ranges required to leave the MINER $\nu \mathrm{A}$ detector. In contrast, most non-muons are Contained prongs. A simple extension of the MINOS-match only muon identification described earlier would be to only accept tracks which are not well-contained in the MINER $\nu \mathrm{A}$ Inner Detector. However, such a selection would have significant non-muon backgrounds which could be reduced by a simple PID, as suggested in this chapter. These categories do allow us to modify our selection of particular types of tracks, which is an option we will take advantage of later, increasing the threshold for Contained prongs, where the backgrounds are much larger than the muon signal.

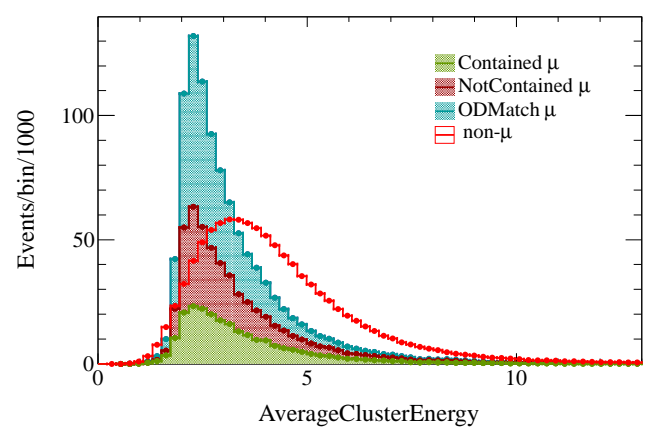

(a) Muons divided by prong type

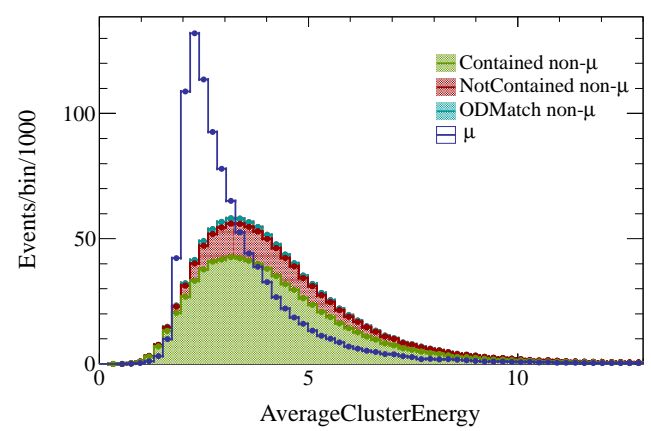

(b) Non-Muons divided by prong type

Figure 9.3: The average cluster energy in the Tracker region with the distributions divided by the last MINER $\nu \mathrm{A}$ sub detector region traversed by the particle.

\subsubsection{Muon Identification Variables in the MINER $\nu$ A Tracker, ECAL and HCAL regions}

MINER $\nu \mathrm{A}$ must construct k-NN input variables with pion-muon separation. This is more difficult for MINER $\nu \mathrm{A}$ because of the non-uniform density 
in the MINER $\nu \mathrm{A}$ detector. Each sub-detector region in the MINER $\nu \mathrm{A}$ detector has a different amount of active scintillator per total mass. The easiest solution is to only use the energy deposited along the track in a particular region. For example, the average energy along a track may be calculated for the same track and a particular sub-detector (i.e. The average energy along the track in the ECAL sub-detector region).

The Tracker region is closest to the upstream target region and is without any heavy materials (except in the frame). Muons typically travel a distance longer than the Tracker region. Hadronic particles are more likely to be contained in the Tracker region because they have less energy and a probability of interacting strongly. Because of its low density, the least amount of energy is lost per track length in the Tracker region and pions are less likely to interact hadronically. Therefore, the separation between muons and nonmuons is smaller for the Tracker region than it is for the heavier, downstream regions.

In Figure 9.4, variables similar to the variables used in the MINOS $\mu$ ID$\mathrm{A}$ are shown for the 'Tracker' region of the MINER $\nu \mathrm{A}$ inner detector. The variables in Figure 9.4 are area normalized. The average cluster energy and signal fluctuation parameter shown in Figure 9.4 for MINER $\nu \mathrm{A}$ are similar to the same variables MINOS variables. The 'End Fraction Energy' is the ratio of the energy in the last $50 \%$ of the track. This variable was used to see if the low-energy upturn in the Bethe-Bloch curve could be seen in the track. The non-muon histogram is colored by the fraction which is composed of a particular background type. The muon identification is most concerned with pion-muon separation. Therefore, the pion fraction of the total background is the most relevant. 


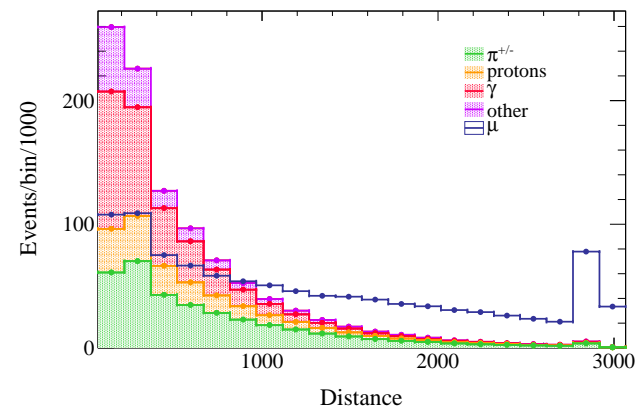

(a) Distance traveled in the tracker (in $\mathrm{mm}$ )

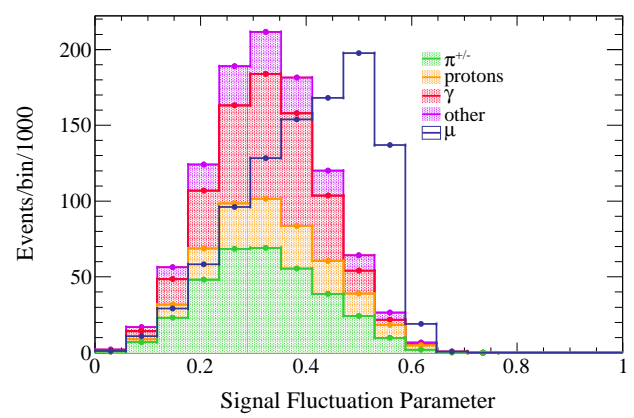

(c) Signal fluctuation parameter in the Tracker Region

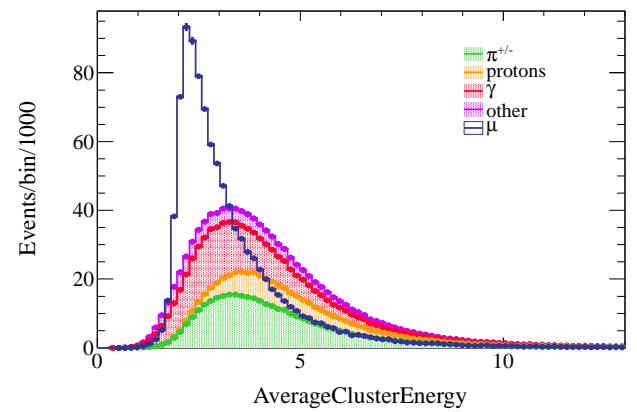

(b) Average cluster energy in the Tracker Region

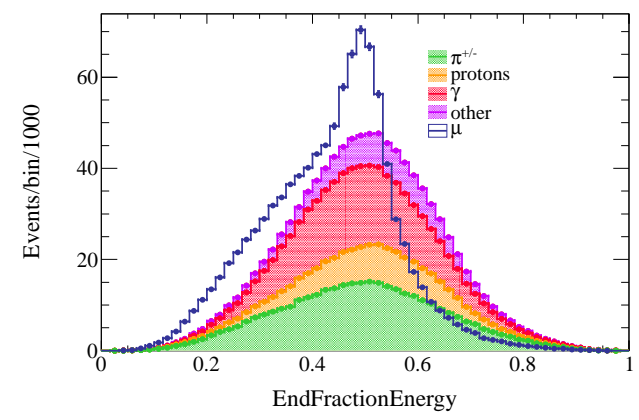

(d) End energy fraction in the Tracker Region

Figure 9.4: Muon identification variables for the MINER $\nu \mathrm{A}$ Tracker region that distinguish between pions and muons. The non-muon histogram is composed of different particle-type histograms stacked together. The histograms are normalized to the same number of muon and non-muon events. Although all non-muon particles are shown, the pions shown in green are the most relevant background. This is because the ionization profile PID can distinguish the other particle types. 
In Figure 9.5, the same four variables which were shown in Figure 9.4 for the Tracker region are shown for the ECAL region. Due to the density and geometry of the ECAL, the behavior of each variable is quite different. Particles which enter the ECAL are more likely to exit the ECAL through the back because the ECAL is located further downstream of the nuclear targets (and the fiducial volume in general). Furthermore, the kinematics of particles which enter the ECAL are sculpted towards small zenith angle and high energies and the ECAL is shorter in length than the Tracker region. Therefore, the distance traveled in the ECAL is not a strong identifying variable. Because of the lead sheets on the ECAL region planes, there is a larger amount of energy lost per plane in the ECAL. This contributes to a larger difference in the 'End Fraction Energy' variable. The 'Signal Fluctuation Parameter' also sees a larger difference between muons and non-muons in the ECAL than in the Tracker region. This is likely caused by the position of the particle in its ionization curve.

Finally, in Figure 9.6 the identification variables are shown for the HCAL region. The HCAL was designed to stop high-energy hadronic particles by using thick steel plates as mentioned in Chapter 3. High-energy muons tend to exit the HCAL. Because the HCAL region is the furthest downstream, most hadronic particles from events originating from the Tracker and Target regions have stopped. Therefore, tracks in the HCAL are overwhelmingly muons.

\subsubsection{Muon Identification Variables in the MINER $\nu \mathrm{A}$ Outer De- tector}

Variables for muon identification in the Outer Detector must differ from the variables described previously due to the different geometry and lack of 


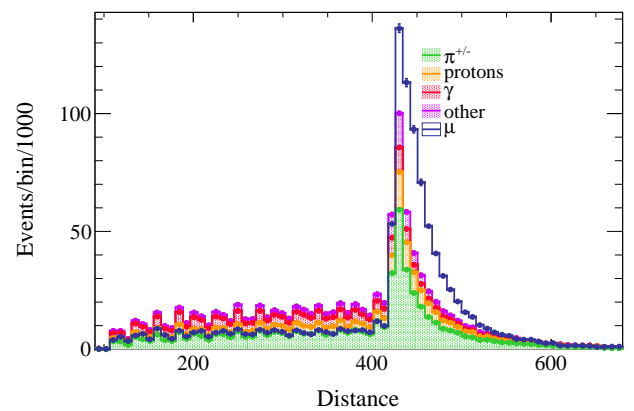

(a) Distance traveled in the ECAL (in $\mathrm{mm}$ )

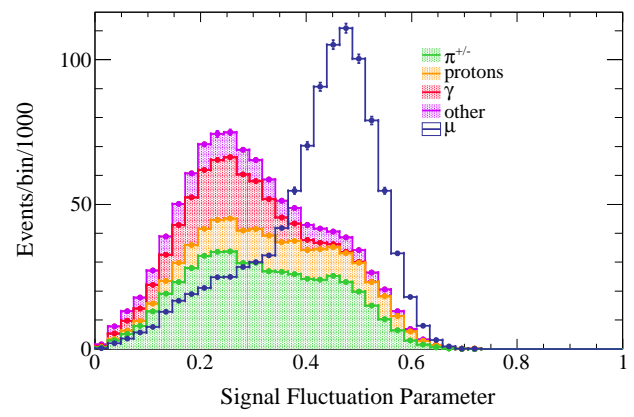

(c) Signal fluctuation Parameter in the ECAL

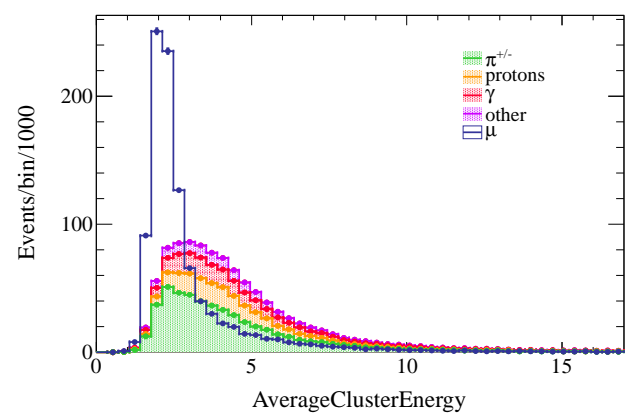

(b) Average cluster energy in the ECAL

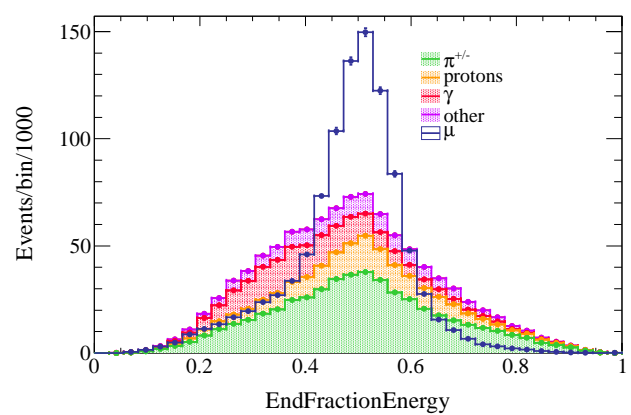

(d) End energy fraction in the ECAL

Figure 9.5: Muon identification variables for the MINER $\nu \mathrm{A}$ ECAL region that distinguish between pions and muons. The non-muon histogram is composed of different particle-type histograms stacked together. The histograms are normalized to the same number of muon and non-muon events. Although all non-muon particles are shown, the pions shown in green are the most relevant background. This is because the ionization profile PID can distinguish the other particle types. 


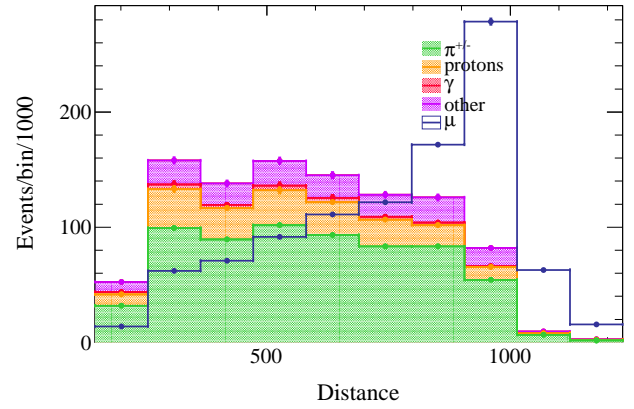

(a) Distance traveled in the HCAL (in $\mathrm{mm}$ )

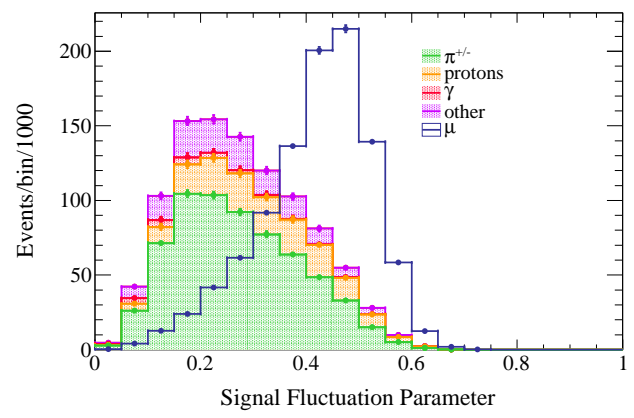

(c) Signal fluctuation parameter

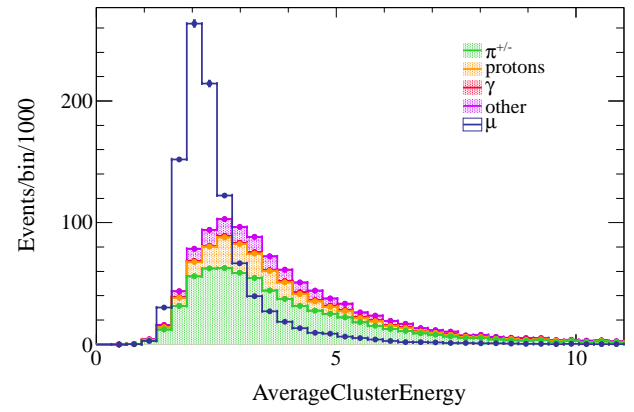

(b) Average cluster energy

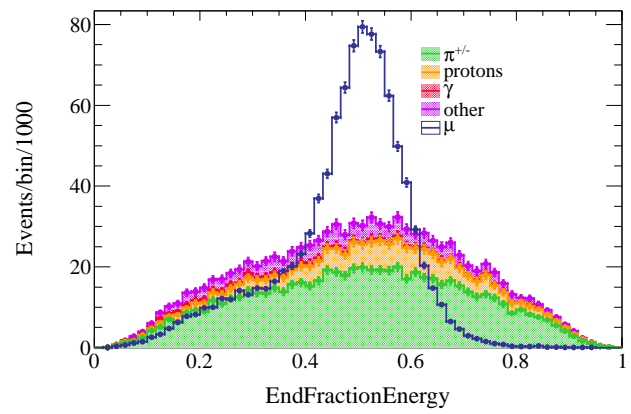

(d) End energy fraction

Figure 9.6: Muon identification variables for the MINER $\nu$ A HCAL region that distinguish between pions and muons. The non-muon histogram is composed of different particle-type histograms stacked together. The histograms are normalized to the same number of muon and non-muon events. Although all non-muon particles are shown, the pions shown in green are the most relevant background. This is because the ionization profile PID can distinguish the other particle types. 
tracking in the Outer Detector. However, the principles of the muon identification are the same: range, minimum ionization, and scattering can define muons from pions. In Figure 9.7 the three variables composed for muon identification in the Outer Detector are compared. These three variables are the "Downstream energy weighted distance", which is the distance of each Outer Detector cluster weighted by the energy of that cluster, the "Energy per cluster", which is analogous to the average energy for the Inner Detector regions, and the "Number of Downstream Stories", which is related to how far into the Outer Detector the particle penetrated. Two of the variables have the qualifier "downstream" attached to recognize the fact that muons which originate from events outside the MINER $\nu \mathrm{A}$ detector also have "upstream" Outer Detector hits, related to the entry of the particle into the MINER $\nu \mathrm{A}$ detector.

\subsubsection{MINER $\nu$ A Region k-NNs and an Aggregate Score for Muon Identification}

A k-NN (again, see Appendix A for more information on the k-NN algorithm) based PID was constructed for each sub-detector region. These four k-NN identifiers were are shown in Figure 9.8 for data and Monte Carlo. The amount of Monte Carlo was weighted such that the total number of Monte Carlo and data events in the MINER $\nu \mathrm{A}$ detector is the same. However, the differences in kinematic distributions between data and Monte Carlo leads to different number of events in each k-NN histogram. Notice that the data and Monte Carlo have large disagreements for the HCAL and Outer detector regions. This presents a challenge for the MINER $\nu \mathrm{A}$ experiment. If the Monte Carlo were correctly modeling the data, the distributions should be the same. However, because of the lack of neutrino multiplicity and exclusive cross-section measurements at low-energy, it is known that the Monte Carlo 


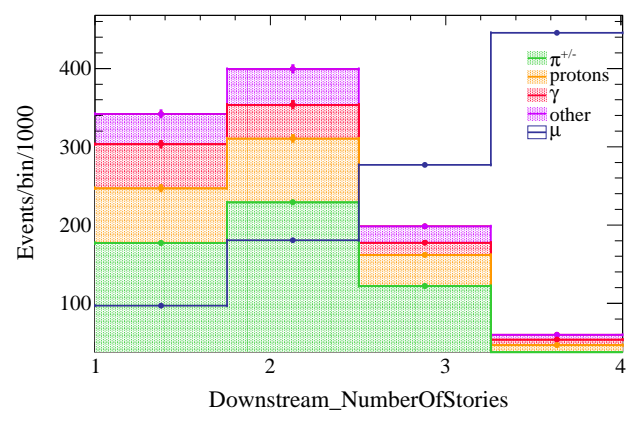

(a) Downstream Number of Stories

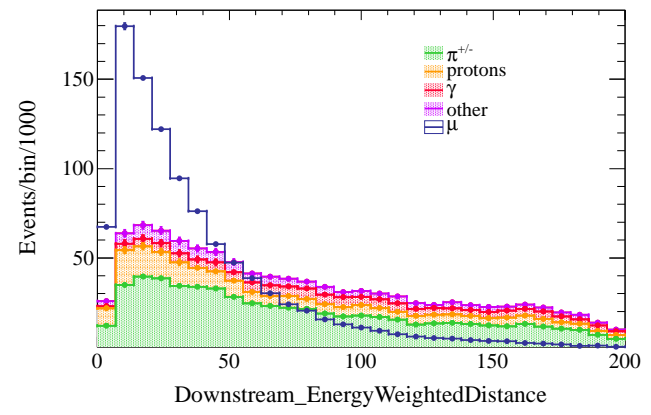

(c) Energy Weighted Distance to the Projected Track

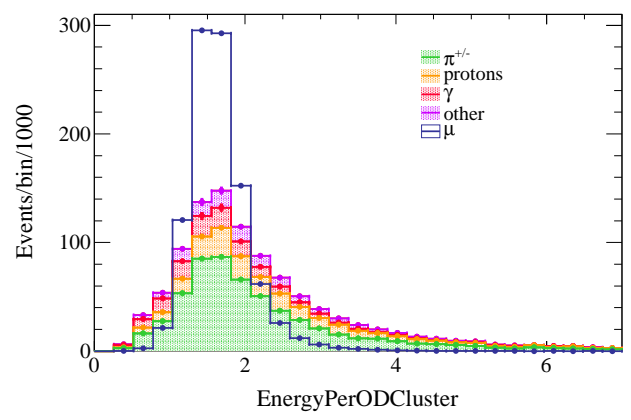

(b) Energy per OD Cluster

Figure 9.7: The MINER $\nu$ A Outer Detector muon identification variables. The number of stories is related to how far the track went radially, the energy per cluster is related to the minimum ionizing nature of muons, and the 'energy weighted density' is the distance of the correlated Outer Detector hits from a track projected into the Outer Detector. The nonmuon histogram is composed of different particle-type histograms stacked together. The histograms are normalized to the same number of muon and non-muon events. Although all nonmuon particles are shown, the pions shown in green are the most relevant background. This is because the ionization profile PID can distinguish the other particle types. 
may contain physics-related errors. However, it is imperative that we agree the Monte Carlo models hadronic particle interactions correctly in the MINER $\nu \mathrm{A}$ detector. This is extremely hard to distinguish from possible physics-related errors.

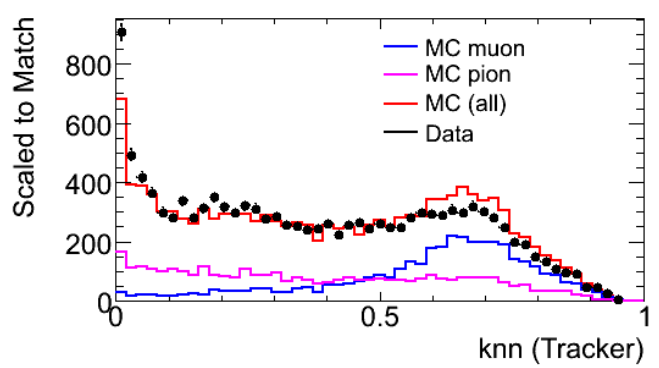

(a) Tracker Region k-NN muon-ID

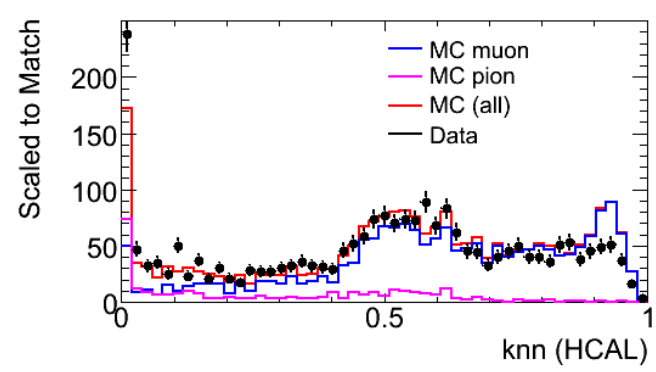

(c) HCAL Region k-NN muon-ID

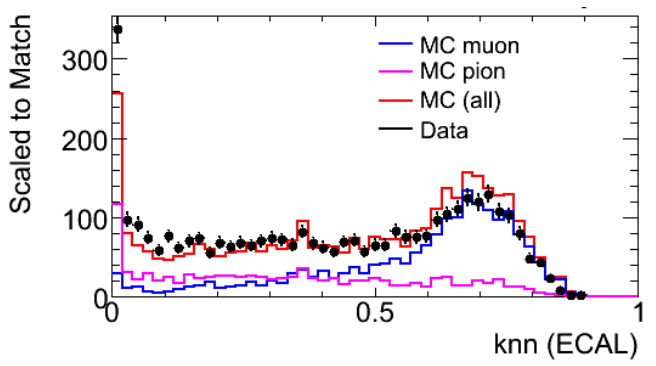

(b) ECAL Region k-NN muon-ID

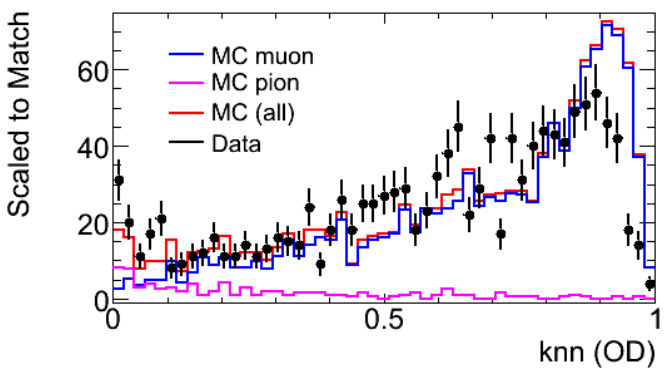

(d) Outer Detector Region k-NN muon-ID

Figure 9.8: The sub-detector k-NN muon identifiers for the MINER $\nu \mathrm{A}$ experiment.

Because the tracks can traverse multiple combination of the four subdetector regions, a 'score' which can function independently of any one of the $\mathrm{k}-\mathrm{NN}$ values is needed. Otherwise, the score would not be defined for particles which only interact in one particular sub-detector region. A muon 'score' was created which is the best of the four k-NN selector values. This was found to give good separation between pions and muons using the four sub-detector k-NNs. The muon score is shown in Figure 9.9. A large discrepancy is seen 
between the data and Monte Carlo. This is just a reflection of the discrepancies seen in the individual k-NN for each sub-detector region. The source of this discrepancy has not been found.

A selection of muon neutrino events for Monte Carlo is shown in Figure 9.10. This selection includes

- All Minos-Match and Minos-Stub prongs

- ODMatch and Not-Contained prongs which have a value of the score greater than 0.5

- Contained prongs which have a value of the score greater than 0.7

This selection has not been optimized and merely stands as an example of the increases in efficiency that can be gained by MINER $\nu \mathrm{A}$. It is clear that it is possible using the muon identification score shown before that the selection of muons at low-energy increases dramatically. This would vastly improve MINER $\nu$ A's current charged current analyses. Additionally, this would help exclusive cross-section analyses distinguish between low-energy pions and muons. The efficiency and contamination of the selection is shown in Figure 9.11. Here, it is apparent that there are some large contaminations, but mostly from particles which can be distinguished using the ionization profile. Most backgrounds come from the Contained prong category. Removing this category reduces the efficiency below $2 \mathrm{GeV}$, but also reduces backgrounds by half. The pion contamination is roughly half the total hadron contamination, according to earlier studies [98]. Additionally efficiency and contamination are highly dependent on the fiducial volume for MINER $\nu \mathrm{A}$; looser fiducial volumes allow more particles which tend to escape the MINER $\nu \mathrm{A}$ detector 


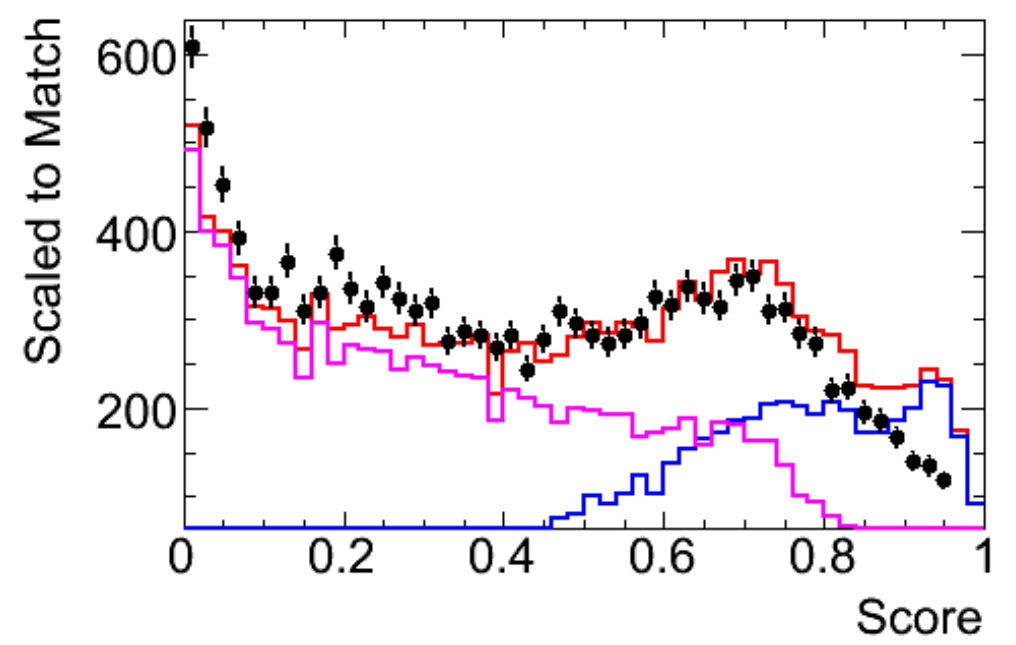

(a) The MINER $\nu$ A score

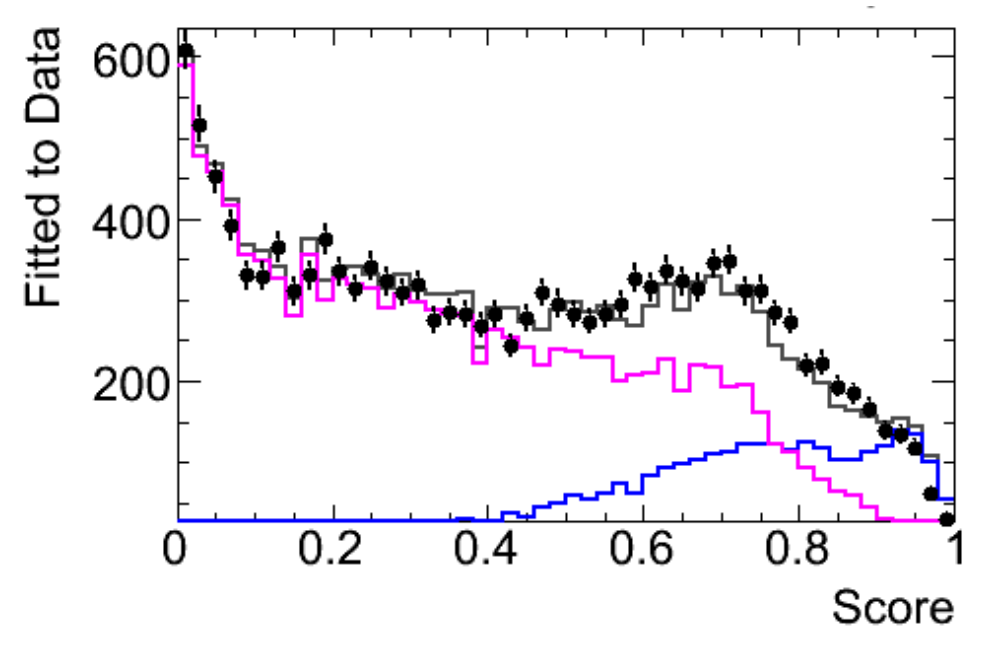

(b) The MINER $\nu \mathrm{A}$ score fitted to data

Figure 9.9: The MINER $\nu$ A muon-identification score. This score is the highest value for any of the four k-NNs (one for each sub-detector region: Tracker, ECAL, HCAL, and Outer Detector). A large discrepancy is seen between data and Monte Carlo at high-PID values. It is unknown whether this is due to detector calibrations, differences in physics models and real neutrino events, or unphysical Monte Carlo errors. 
and are harder to identify. By reducing the selection to only events from the Target or Tracker regions of the inner detector, one can increase the purity of the sample for the same efficiency [98].

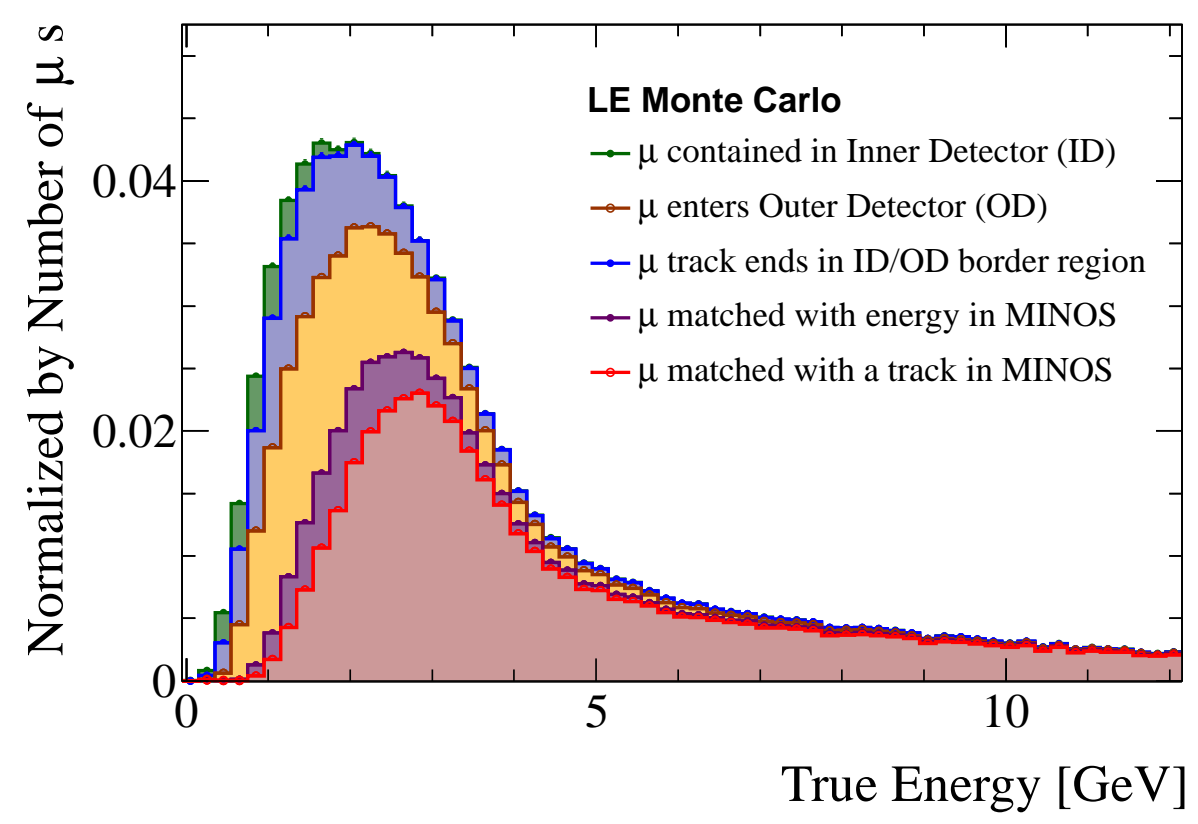

Figure 9.10: The muon energy spectrum after a k-NN based muon selection. This is split into the different prong categories a muon can have. All MinosMatch and Minos-Stub prong muons are included in this selection. ODMatch and Not-Contained prong muons which have a score greater than 0.5 are also included. Contained prongs with a score greater than 0.7 are the last (green) category. This histogram is stacked. 


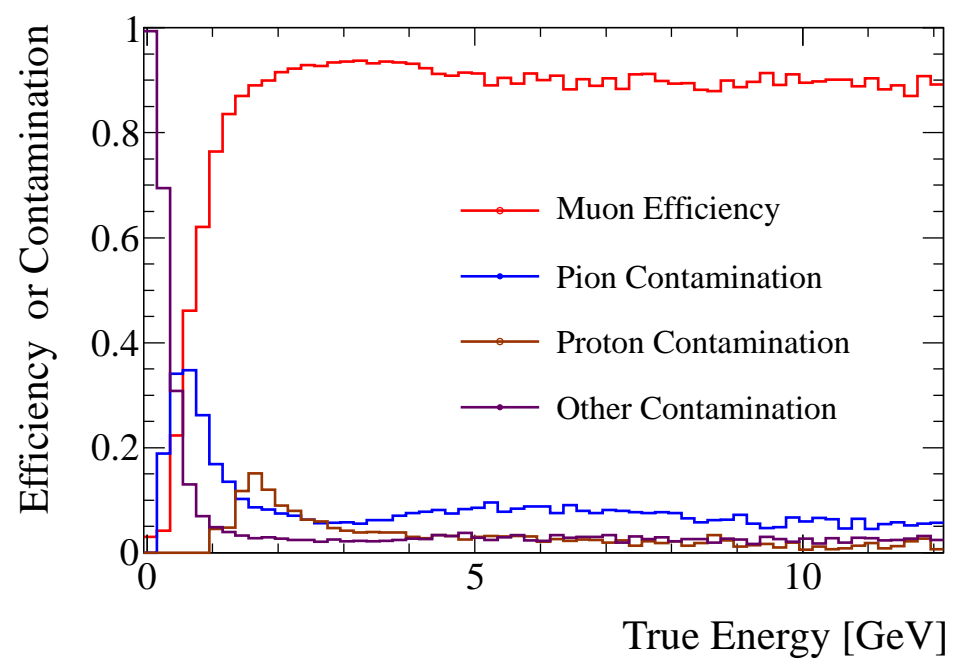

(a) Efficiency and Contamination

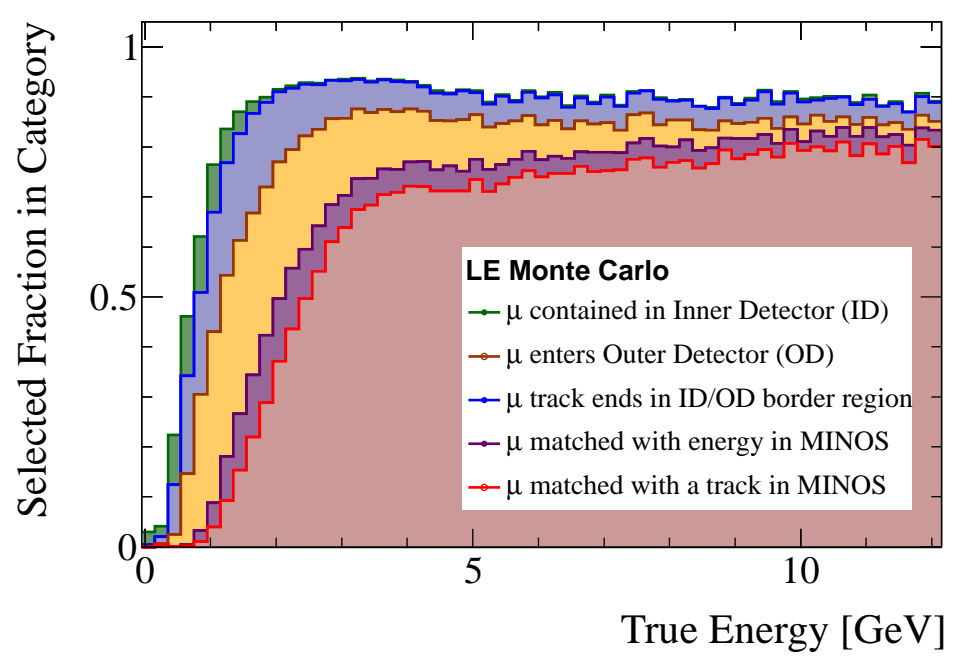

(b) Efficiency broken down by track end-point

Figure 9.11: The efficiency and contamination of a MINOS-like muon particle identifier. 


\subsection{Muon Energy Estimates for the MINER $\nu$ A Exper- iment}

The MINER $\nu \mathrm{A}$ detector was designed and placed in order to take advantage of the muon spectrometer qualities of the MINOS Near Detector. The magnetic field and high density of the MINOS detector make estimates of the muon energy easy and accurate. This can be seen in Figure 9.12, where the dif-

ference between the true and reconstructed energy for Minos-Matched muons is shown. The Minos-Stub classified muons have similar energy reconstruction because the range of the muon is well known. The energy is computed using the same range and curvature algorithms used in MINOS.

One of the major challenges in incorporating events with muons in categories besides Minos-Match and Minos-Stub is energy reconstruction. For example, as mentioned earlier, MINER $\nu$ A could easily increase its charged current selection by including events with ODMatch muons with no other muon selection. However, while Minos-Match and Minos-Stub muons have good energy reconstruction through estimates of their range in MINOS, ODMatch muons, have no such handle through which to estimate the muon energy; they exit the MINER $\nu \mathrm{A}$ detector before ranging out. A crude energy estimate can be made by considering the outer most story of the associated Outer Detector clusters. This estimate is shown in Figure 9.13, which shows the true muon energy divided by which story the last associated cluster is located on. Muons which reach the back of the MINER $\nu \mathrm{A}$ detector result in a high-energy tail for each story. The low-energy threshold for each distribution is dictated by the amount of material that a muon must travel through in order to reach this Outer Detector. A better estimate might be made by considering the total amount of material traversed by the particle. This is being investigated by 
Tammy Walton [99].

For the last two categories of prongs, Contained and Not-Contained, there is no other handle form muon energy estimates besides the ionization profile method described previously. For Contained prongs, this method has been shown to give good estimates of the particle energy. Not-Contained prongs suffer from the same range issues as ODMatch prongs. These categories of muons will likely always have poorer energy resolution. An estimate can be made based on whether the muon is projected to intersect the MINOS detector but does not. However, in general, the MINER $\nu \mathrm{A}$ experiment must use the ionization profile to estimate the energy of such muons.

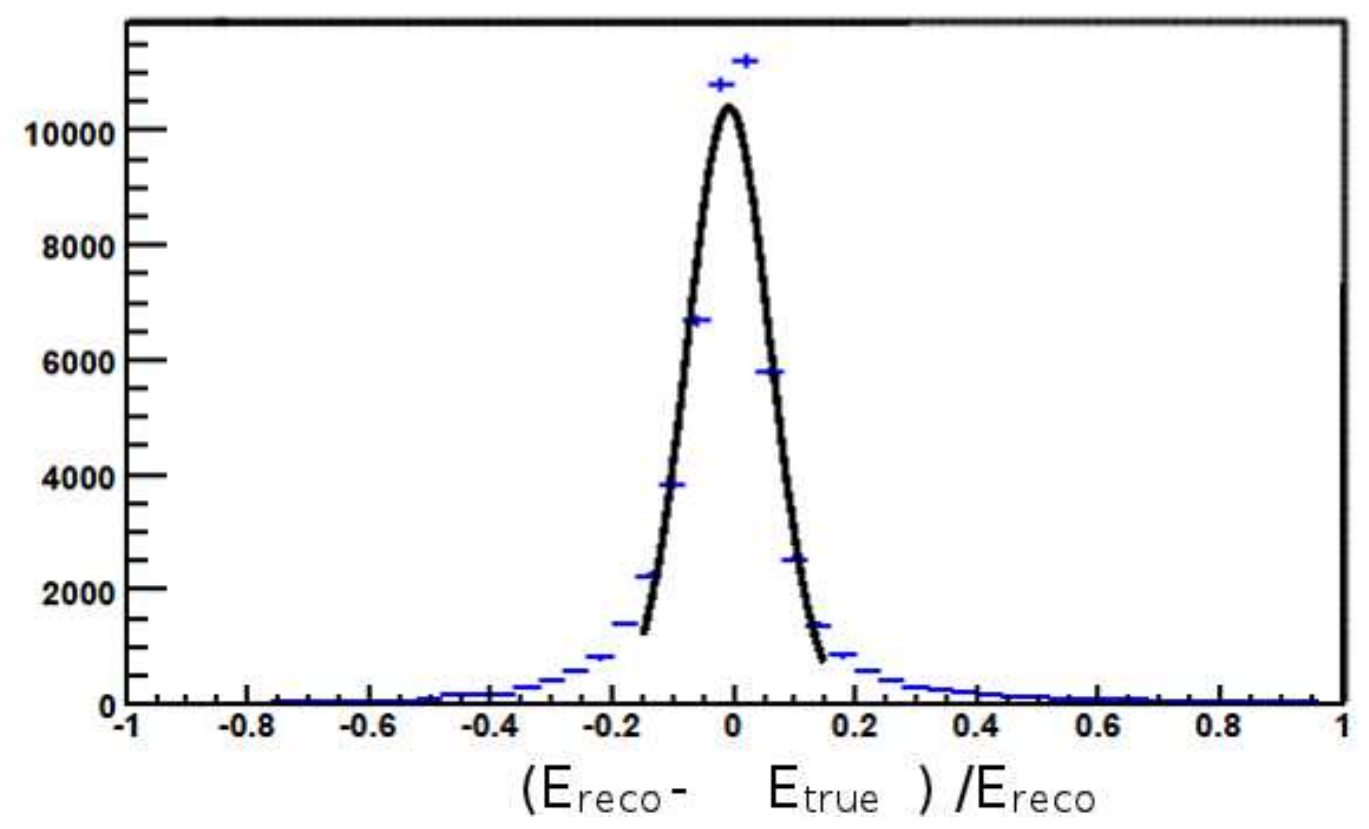

Figure 9.12: The percent difference between reconstructed and true energy for MINOS matched MINER $\nu \mathrm{A}$ muons. The black line represents a Gaussian fit to this Monte Carlo data. . This figure is courtesy of Tammy Walton [99]. 


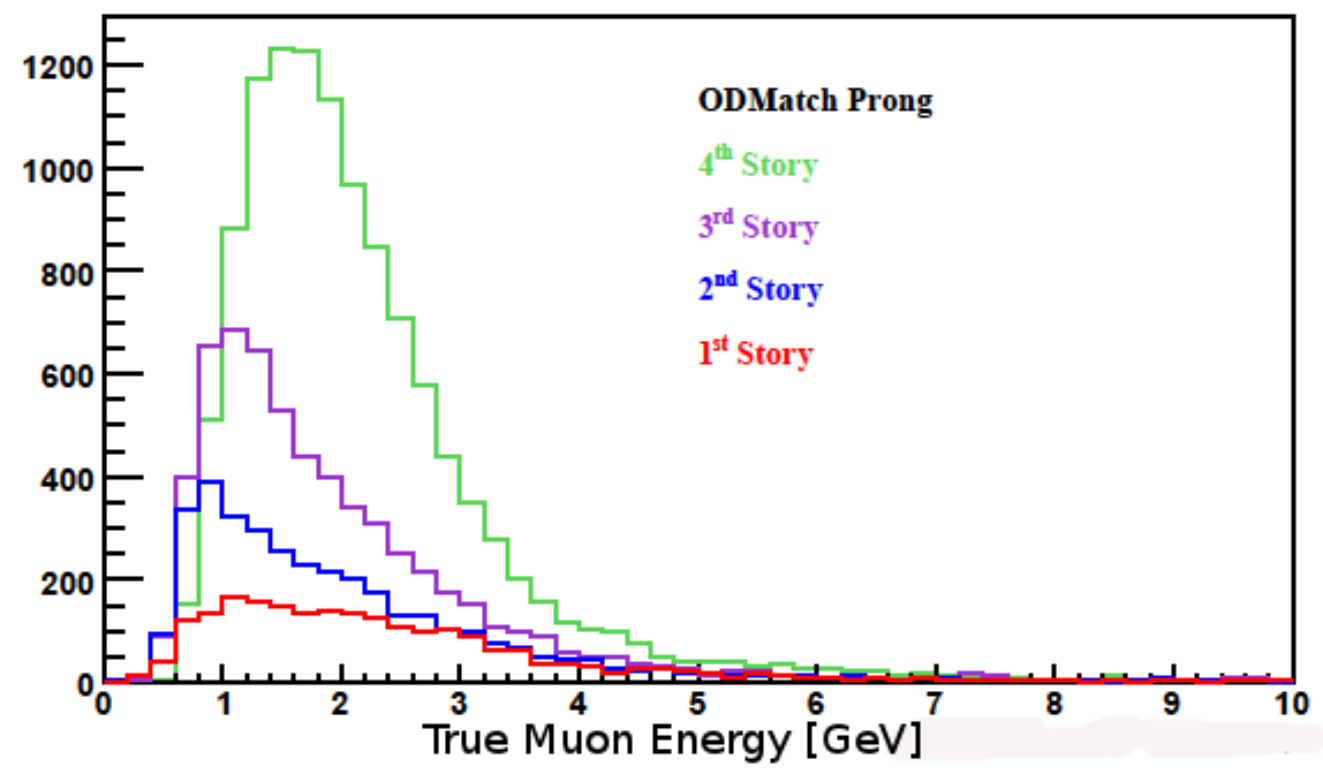

Figure 9.13: The energy of ODMatched muons divided by the last story of the associated OD clusters. By dividing the ODMatch muon sample by story, we can determine the energy of the particle to some degree. Muons which reach the back of the MINER $\nu \mathrm{A}$ detector result in a high-energy tail for each distribution. The low-energy threshold for each distribution is dictated by the amount of material that a muon must travel through in order to reach this Outer Detector. This is essentially a measurement of range. This figure was modified from a talk by Tammy Walton [99]. 


\subsection{Future Relevant MINER $\nu$ A charged current studies}

Nuclear-effect ratios, for instance the charged current event rate ratio of iron to lead, are less affected because some of the efficiency sculpting effects should cancel. Therefore, short-term, MINER $\nu$ A will concentrate on cross-section ratios until the muon selection and reconstruction and (as importantly) flux reweighting and errors can be determined. However, for any analysis which hopes to utilize the more than $50 \%$ of the charged current events from the NuMI LE010185N configuration, or is concerned with the kinematic distribution of its events, including Not-Contained and ODMatch muons is critical. This includes all absolute cross-section measurements. Because absolute cross sections are a crucial component to the success of the MINER $\nu \mathrm{A}$ experiment's goal of precision neutrino cross-section measurements, the inclusion of Not-Contained and ODMatch muons will be a crucial step toward the completion of future MINER $\nu \mathrm{A}$ measurements. In fact, the flux predictions which are critical to MINER $\nu$ A's success need a clean and efficient selection of charged current muon neutrino events in order to reweight the Monte Carlo predictions and produce errors in a manner similar to what was done for MINOS (see Chapter 2 and the work by Zarko Pavlovic [48]).

Besides cross-section measurements, MINER $\nu \mathrm{A}$ is poised to do another analysis, which is simpler, but physically important. This is the measurement of multiplicity in neutrino interactions. This is due to MINER $\nu$ A's unique design with multiple heavy target materials. Furthermore, unlike recent neutrino experiments which have sacrificed image resolution for detector mass, MINER $\nu \mathrm{A}$ has large regions of scintillator-only planes. This allows MINER $\nu \mathrm{A}$ to resolve the multiple particles in a hadronic shower. MINER $\nu \mathrm{A}$ can go further than merely counting the number of interactions of a particular final state. 
It can resolve the energy and momentum distributions of the hadronic particles as well. Such measurements will aid in understanding patronization and nuclear distributions and improve the values quoted in simulations and reduce the error on shower energy estimations.

The MINER $\nu$ A detector's high resolution design is also the source of many reconstruction challenges. The high resolution results in reconstructed events with significantly more complexity that the simple topologies found in MINOS. This was part of the design of MINER $\nu$ A. However, reconstructing such complex topologies can be difficult. Specifically, MINER $\nu$ A often encounters the following topological problems due to the high resolution.

- Backwards going tracks - In MINOS, the image resolution is so poor that a hadronic shower looks like a 'blob'. Because of the low density of the MINER $\nu$ A detector, some backwards-moving particles can have significant track lengths. The vertex of events with backwards-moving particles is much harder to distinguish. Furthermore, backwards-going tracks must be identified in order for the ionization profile particle identification technique to work.

- Secondary interactions - MINER $\nu \mathrm{A}$ events often have secondary interactions: charged pions decaying into muons, neutral pions decaying into photons, etc. Such interactions can produce objects which are the combination of two particles. This can disrupt the ionization profile and complicates the IPPID algorithm.

- 'Kinked' tracks - These are secondary interactions where the initial and final particle are the same. Hadronic particles can often have single nuclear interactions which disrupt the momentum and energy of the 
particle. Such particles appear as two tracks with different slopes, but a common vertex.

In order for the MINER $\nu \mathrm{A}$ experiment to have the significant physics results which MINER $\nu \mathrm{A}$ aspires to, muon identification and energy reconstruction must be addressed. The studies shown in this chapter are merely a first step to much more comprehensive analyses which will be completed in the future. 


\section{Chapter 10}

\section{Summary and Conclusions}

\subsection{Summary}

This thesis designed and improved on methods of identifiying charged current numuevents through determining the presence of a muon in two calorimetric neutrino experiments. We found that even small improvements to the charged current selection can affect oscillation and cross-section measurements.

The MINOS experiment was designed to identify muons from charged current interactions where the muon track-length exceeded roughly 20 planes. Low-energy, short tracked muons are particularly difficult to identify and distinguish from high-energy hadronic particles. In Chapter 4 a new charged current/neutral current separation parameter ( $\mu \mathrm{ID}-\mathrm{B})$ was developed to distinguish low-energy muons from hadronic particles. This new method of capturing low energy charged current events relied on distinguishing hadronic particles through their end-of-life interactions and scattering throughout the track. A new estimate of the neutral current background error based on muon removed charged current events was developed. This was described in Chapter 5 and reduced the related systematic error on the MINOS $2010 \nu_{\mu}$ disappearance analysis measurement of the oscillation parameters. With this reduced systematic error, MINOS was able to modify the charged current selection for the $2010 \nu_{\mu}$ disappearance analysis and increase the number of low-energy neutrino events. This resulted in an extra 8 out of 38 events below $2 \mathrm{GeV}$ 
at the Far Detector and helped resolve the complete oscillation period. This MINOS analysis was discussed in Chapter 7 and Chapter 8.

In Chapter 9, the methods developed in the MINOS experiment were applied to the MINER $\nu \mathrm{A}$ experiment. MINER $\nu \mathrm{A}$, generally has a higher resolution at low-energies than MINOS due to the increase in active material. Preliminary studies show that a MINOS-like muon selection increases the the charged current selection efficiency significantly at low muon energies. Such studies proved that muons which do not enter the MINOS Near Detector are a significant portion of the potentila MINER $\nu \mathrm{A}$ charged current event sample. MINER $\nu \mathrm{A}$ cannot ignore such muons. Further studies must be completed to develop an energy estimate for muons which are not contained in the MINER $\nu \mathrm{A}$ detector. This is significantly harder than it is in MINOS because of the lack of a magnetic field and the variability in the detector density.

\section{$10.2 \quad$ Future Neutrino Measurements}

The anticipated results from the MINER $\nu \mathrm{A}$ experiment include crosssection measurements that are important to neutrino experiments which use few-GeV muon neutrinos. In particular, the $\mathrm{NO} \nu \mathrm{A}$ experiment (NuMI Off-axis $\nu_{e}$ Appearance Experiment) is sensitive to the neutral current $\pi^{0}$ cross-section. $\mathrm{NO} \nu \mathrm{A}$ is an experiment which is centered off-axis of the NuMI beam in order to measure an excess of $\nu_{e}$ events as predicted by neutrino oscillations. This is a 2nd-order oscillation effect, as the main transition for $\nu_{\mu} \mathrm{s}$ in the NuMI beam is from $\nu_{\mu}$ to $\nu_{\tau}$. Therefore, the $\nu_{e}$ signal is very small and overwhelmed by backgrounds, such as neutral current $\pi^{0}$ events, which are indistinguishable from the $\nu_{e}$ signal. In Figure 10.1, a signal $\nu_{e}$ event and a neutral current event with an electromagnetic shower (likely from a $\pi^{0}$ decay) are shown. Note the 


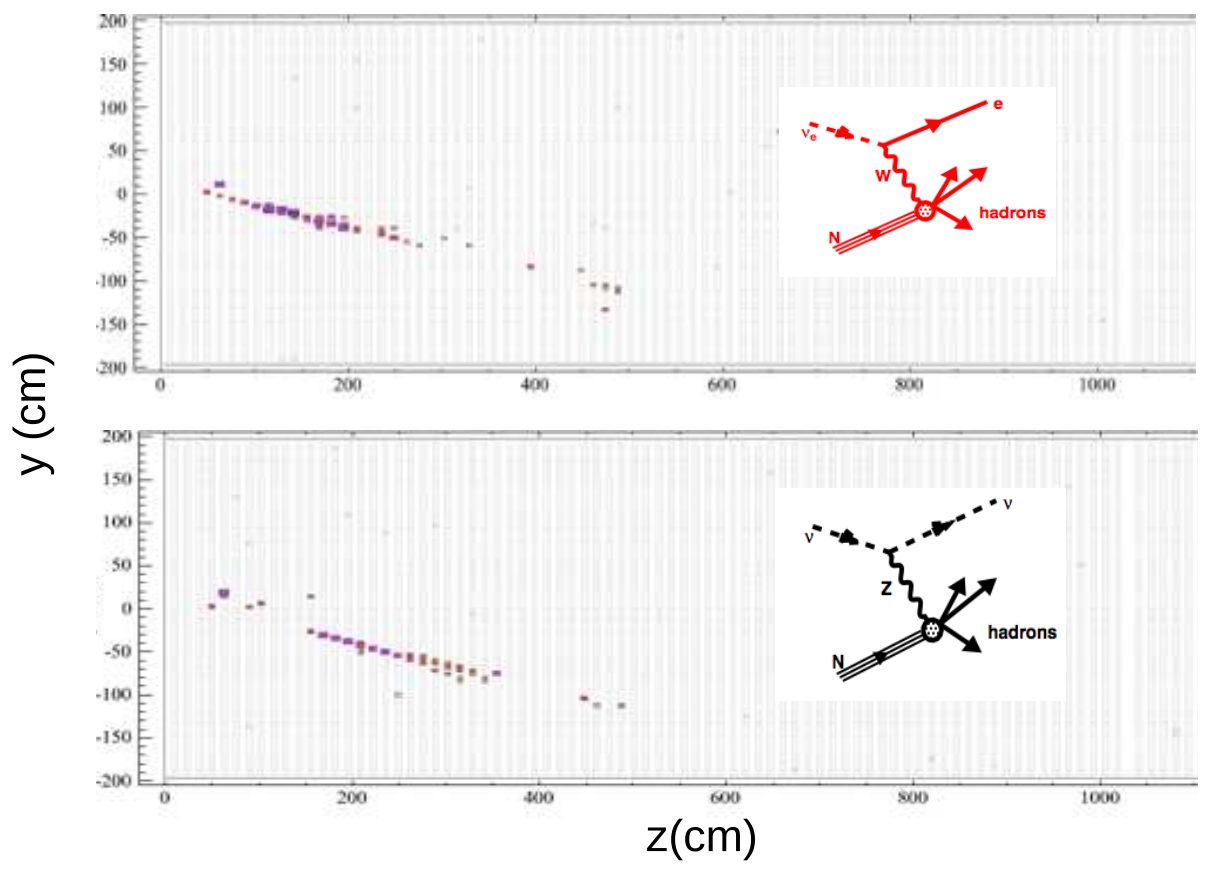

Figure 10.1: Two event displays for the $\mathrm{NO} \nu \mathrm{A}$ experiment. The top is a signal $\nu_{e}$ charged current event with an electromagnetic shower. The bottom is a background $\nu$ neutral current $\pi^{0}$ event with a similar electromagnetic shower. This irreducible neutral current background means that the neutral current cross-section is particularly important to the $\mathrm{NO} \nu \mathrm{A}$ experiment. This is adapted from P. Vahle's presentation [100].

similarity between the two event topologies. Figure 10.2 shows the expected event rates for the $\mathrm{NO} \nu \mathrm{A}$ experiment, which includes a large neutral current background.

In order for a successful result from $\mathrm{NO} \nu \mathrm{A}$, the cross-section of neutral current $\pi^{0}$ events at low energies must be known well. Therefore results from experiments like MINER $\nu \mathrm{A}$ (and the similar experiment, $\mathrm{SciNO} \nu \mathrm{A}$ [101]) are desired by the neutrino community at large. Low-energy muon identification is crucial for effective neutral current and charged current event identification 


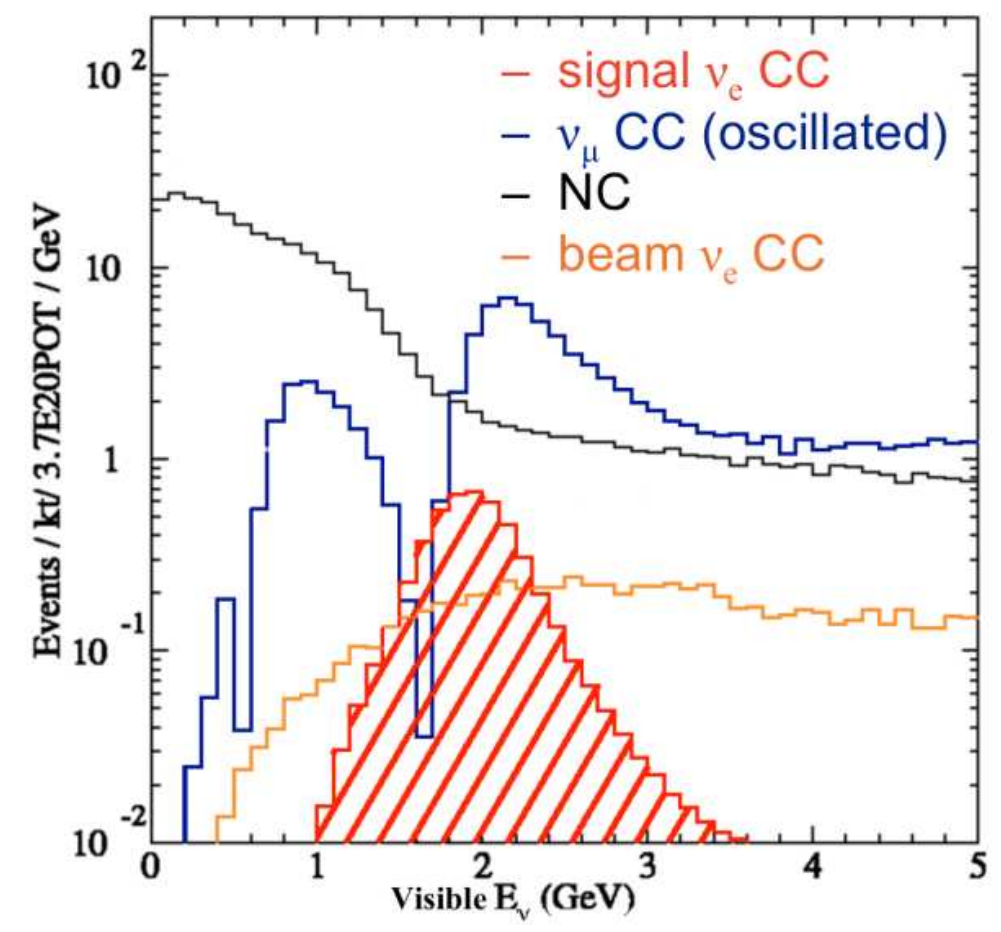

Figure 10.2: The expected event rate for $\mathrm{NO} \nu \mathrm{A}$. Note the large neutral current background. An portion of neutral current events will create a $\pi^{0}$ in the final state. This can mimic the $\nu_{e}$ appearance signal. Taken from P. Vahle's presentation on $\mathrm{NO} \nu \mathrm{A}[100]$. 
at the energies relavent to $\mathrm{NO} \nu \mathrm{A}$. Therefore, understanding low energy muons is increasingly important to neutrino experiments. The lack of a magnetic field or high-density materials in the MINER $\nu \mathrm{A}$ tracker region, and the variablility of the MINER $\nu \mathrm{A}$ detector density increases the need to understand muons in MINER $\nu \mathrm{A}$. We expect that muon studies will be an important component to the MINER $\nu \mathrm{A}$ experiment in the future.

\subsection{Final Thoughts on Neutrinos}

From the birth of the neutrino as a solution to energy conservation in beta decay to recent oscillation experiments, neutrinos have continuously exposed unexpected phenomena in particle physics. The explanations for these phenomena, from the description of the neutrino as a mass-and-chargeless lepton to neutrino mass-flavor mixing, are seemingly simple. However, when one probes deeper, these explanations by themselves fall short. For instance, there is still no explanation for why the neutrino mass is so small, or what effect leads to near maximal mixing $\left(\theta_{32} \approx \pi / 4\right)$ in neutrinos.

At a basic level, neutrino oscillations are not well understood. The analogous 'perturbative hamiltonian' for neutrino mixing is unknown. This simply reflects the fact that mass is not well understood. Indeed, at first glance, neutrino oscillations appear to violate energy and momentum conservation (ironically, the same quantities that the neutrino's existance once solved). Recently, these aspects of neutrino oscillations have gained more interest by the physics community. ${ }^{1}$

\footnotetext{
${ }^{1}$ The OPERA measurement of faster-than-light neutrinos may also suggest that the traveling neutrino momentum or energy eigenstates are much more interesting than previously assumed [102]. However, this result is far from verified.
} 
Neutrinos and neutrino oscillations are far from well understood. Their properties hint at rich physics yet to be discovered. The next generation of experiments on the NuMI beamline, like MINER $\nu \mathrm{A}$, and $\mathrm{NO} \nu \mathrm{A}$ are sure measure new and interesting aspects of neutrino physics. However, a crucial part of these experiments will be the identification of the muon through similar means as is used in MINOS and is currently being developed for MINER $\nu$ A. When the $\mathrm{NO} \nu \mathrm{A}$ high-energy running era begins, new challenges will emerge, just as the different design between MINOS and MINER $\nu$ A presented new challenges for muon identification. 
Appendices 


\section{Appendix A}

\section{Using a k-Nearest Neighbor Method for Identification and Extrapolation}

The MINOS experiment uses a k-Nearest Neighbor (k-NN) method $[69,103-105]$ in both the separation of charged current and neutral current events (as described in Chapter 4) and in estimating the shower energy [81]. This appendix describes the k-NN method generally, its use as both a tool for classification and interpolation, and the performance of the $\mathrm{k}-\mathrm{NN}$ as a tool for classification.

\section{A.1 Description of the k-NN Algorithm}

The k-NN algorithm is a method of classifying objects (in this case,

physics events) based on the known classification of nearby objects in the feature space. It is a type of memory-based learning, comparing an object of unknown classification to those with known and stored in memory.

The k-NN algorithm is described by the following steps.

1. Begin with a set of objects whose type, $\alpha$, has been determined and whose values for the set of variables $\vec{x}=\left(x_{1}, x_{2} \ldots x_{n}\right)$ can be determined. This is called the 'training sample'. The set of variables define a n-dimensional space called the feature space. For the MINOS and MINER $\nu$ A experiements, the $\mathrm{n}$ features are the input variables, which have separation 
between muon and non-muon type particles. It is generally desirable to have a mechanism which will allow for easy searching of the feature space near a given point. This is the purpose of the KDTree object which will be described in the next section.

2. An unclassified event from a set of unclassified events known as the 'testing sample' with a position in the feature space given by $\vec{q}=\left(q_{1}, q_{2}, \ldots q_{n}\right)$ is introduced. In order to determine the likely type of this event, the algorithm searches the feature space near the position $\vec{q}$, where the distance to this event is usually calculated using the Mahalanobis distance

$$
d(\vec{q}, \vec{x})=\sqrt{(\vec{q}-\vec{x}) \mathbf{S}^{-1}(\vec{q}-\vec{x})}
$$

but in the MINOS and MINER $\nu \mathrm{A}$ experiments is calculated by using the reduced Euclidean distance, and the covariance matrix is replaced with a diagonal matrix whose elements represent the $80 \%$ cover region of the variables (ie: $80 \%$ of the entries in the variable are within this range).

3. The $\mathrm{k}$ nearest objects 'neighbors' (hence the name k-nearest neighbor) are queried about their type, $\alpha$. The underlying logic behind the k-NN algorithm is :

Theorem A.1.1. If all event types are considered in the construction of the feature space, then $\vec{q}$ must have a value of $\alpha$. The probability that the unclassified event has a type $\alpha=0$ is proportional to the fraction of nearby events which also have a type $\alpha=0$,

$$
\theta=\frac{n_{\alpha=0}}{k}
$$


This fraction is the product of the k-NN algorithm for classification, where $n_{a l p h a=0}$ is the number of nearest neighbors with type $\alpha=0$ and $k$ is the number of events closest to the point $\vec{q}$ used.

When the k-NN algorithm is used for interpolation, such as for the shower-energy estimator, the discrete type, $\alpha$, is replaced with a continuous variable and the fraction $\theta$ is replaced with an average of the nearest-neighbor values for $\alpha$,

$$
\alpha_{\text {estimate }}=\frac{1}{k} \sum_{i=0}^{k} \alpha_{i}
$$

The k-NN fraction can also be weighted by the distance to the point, although this has been shown to have little benefit except for low-statistics training samples.

$$
\alpha_{\text {estimate }}=\frac{1}{\sum_{i=0}^{k} D\left(\vec{x}_{i}-\vec{q}\right)} \sum_{i=0}^{k} \alpha_{i} \times D\left(\vec{x}_{i}-\vec{q}\right) .
$$

In Figure A.1, a visual example of a k-NN method is shown. In this case, there are two types of events ( 0 and 1 ) which have two variables that define a 2-D feature space. Three points are shown as stars among the triangular 'training sample' points. This particular example is symmetric around the $x=y$ line, but the number of events on the $x<y$ side is populated with 10 times fewer events. Test point A and Test point B are symmetrically placed about the $x=y$ line at $\vec{A}=(0.5,1.0)$ and $\vec{B}=(1.0,0.5)$. These points are near an increase in $\alpha=1$ (Type 1 ) events. Test point $\mathrm{C}$ is solidly in a region dominated by $\alpha=0$ (Type 0 ) events $(\vec{C}=(0.2,0.2))$. However, there is an increase in $\alpha=1$ events a moderate distance away. The black rings about these three points represents a radius of 0.1 and 0.2 from the center points. Overall, there are 8000 training events equally divided into the two groups. 


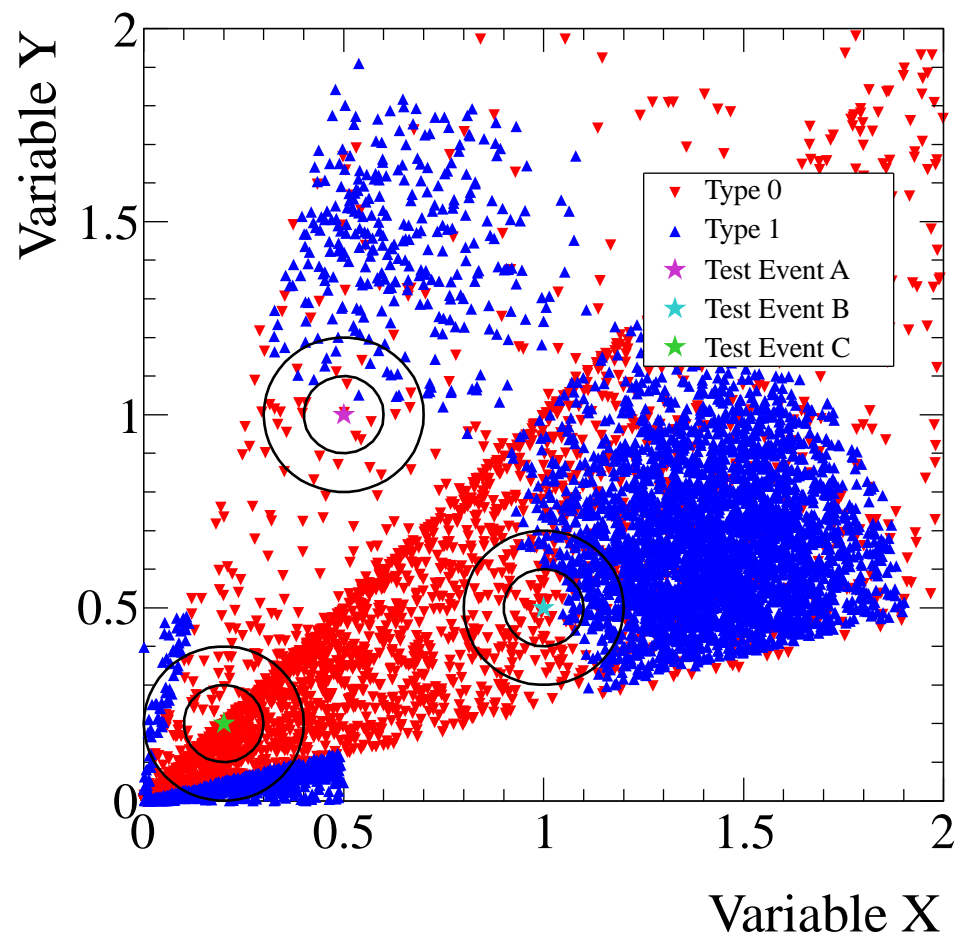

Figure A.1: k-Nearest-Neighbors: a 2-Dimensional example with large and small features. random points from two algorithms (red and blue triangles) occupy a 2-Dimensional feature space. Three example queries are used. Test point (A) and test point (B) are near a large feature. Test point $(\mathrm{C})$ is near a small feature. 
Event $\mathrm{C}$ should be easily identifiable as a $\alpha=0$ event because its nearby neighbors are overwhelmingly $\alpha=0$ events. Events A and B are less certainly $\alpha=0$ events. Compared to $\mathrm{B}, \mathrm{A}$ is in a region of low statistics but a similar distribution of events. One could using the fraction of $\alpha=0$ training events to classify these events in two ways. First, one could use the k-NN method and query the k closest training events. However, one could also conceive of using all training events within a specified distance of the test event. In the limit of using all training events or, equivalently, an infinite radius, the value of the ratio is $\theta 0.5$. This is because the total number of $\alpha=0$ and $\alpha=1$ events are equal. Therefore, in order for the ratio to be useful, we must limit our search to a particular value of $\mathrm{k}$ (in the case of the $\mathrm{k}-\mathrm{NN}$ algorithm) or restrict the training events to those within a certain distance from the test point.

In Figure A.2, the the fraction of events with $\alpha=0$ near a given testing event is compared to the radius of the furthest event queried and the number of events queried. For instance, there are five training events within a radius of 0.1 from test event A. Only one of these events is of type $\alpha=1$. Thus, the fraction of $\alpha=0$ events is $n_{0} / l=4 / 5=0.8$. Therefore, the fraction is 0.8 at a distance of $R=0.1$, and number of nearest neighbor value of $k=5$. The errors on each plot are binomially calculated errors of the ratio, $\sigma_{\theta}=\sqrt{(\theta(1-\theta) / k)}$.

$\mathrm{C}$ retains a value of $\theta=1.0$ until approximately $R \approx 1.5$. At this point a sudden decrease in $\theta$ is seen. This is because of the $\alpha=1$ feature a particular distance away from test event $\mathrm{C}$. The fraction is affected by clearly non-local effects. Therefore, at this radius, we can assume that the fraction $\theta$ is no longer a good representation of average value at point $\mathrm{C}$. One can see similar effects on test event $\mathrm{A}$ and $\mathrm{B}$ at different values of $R$. More importantly however, 

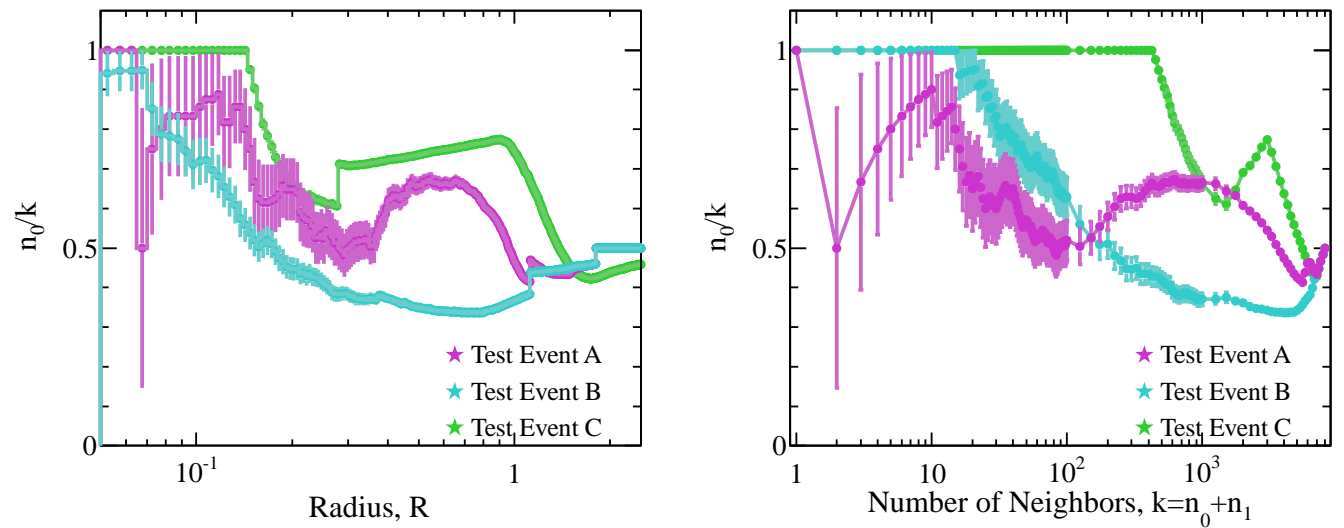

Figure A.2: Left: the fraction of events with $\alpha=0$ near test events A, B, and $\mathrm{C}$ versus the distance to the furthest training event queried. Right: the fraction of events with $\alpha=0$ near test events $\mathrm{A}, \mathrm{B}$, and $\mathrm{C}$ versus the number of nearby events queried (the k-NN algorithm).

note that the value of $\theta$ for test event $A$ is always higher than test event $B$ for the same radius. This would seem incorrect considering test event $\mathrm{B}$ is in a statistically higher region where it is more apparently in a region dominated by $\alpha=0$ events. Furthermore, the errors on these values are quite high for values of $R<1.5$. In contrast, one can see in Figure A.2 that the fraction of $\theta$ versus the number of nearest neighbors does not suffer from these problems. There exists a value of $k$ where test event $\mathrm{C}$ has a sufficiently high value of $\theta$, test events $\mathrm{A}$ and $\mathrm{B}$ are statistically sound, and the value of $\theta$ is higher for test event B than for test event A.

\section{A.2 Running Time and the k-D Tree}

The k-NN algorithm, as well as other data-mining algorithms performs best with large amounts of data. Because the data is multi-dimensional it 
cannot be easily ordered in arrays or other linear containers.

The $\mathrm{k}$-D tree (which is short for k-dimensional tree) is a popular method of reducing the problem of searching a particular region in a high-dimensional space. The k-D tree is a binary tree where each node represents a k-dimensional point. That is, each node represents the data of one event. Binary trees are the simplest tree data structure, with each node having pointers to two daughter nodes such that each node divides the remaining space in a particular dimension.

The splitting dimension alternates with each progressive node. An example of a k-D Tree for two-dimensions is shown in Figure A.3. The binary tree in Figure A.3 is shown visually as a 2-D plot of points. Lines indicate where how a particular node partitions the 2-D space. For instance, the line at $(\mathrm{x}=0.9)$ represents the top node (often called the 'root' node), which partitions the events into two groups with events with $x<0.9$ to the left and those with $x \geq 0.9$ to the right. Similarly, there are horizontal blue lines at $x<0.9, y=$ 1.3 and $x>0.9, y=0.8$ which represent the partitioning done by the daughter nodes of the root node (seen on the second level of the tree).

A box, representing a test point at $(x=1.6, y=1.4)$ is also shown with a dotted circle encompassing the closest five points. A search of the $\mathrm{k}$ nearest neighbors in two-dimensions would progress in the following way:

1. Begin at the top-most root node. Calculate whether the test point is likely higher or lower in the X-dimension. Repeat for successive nodes until you reach a 'leaf' node with no daughters. The closest k nodes to the test point are saved in an ordered container. For the specific case shown in Figure A.3, the last node would be $(1.9,1.6)$ and the 

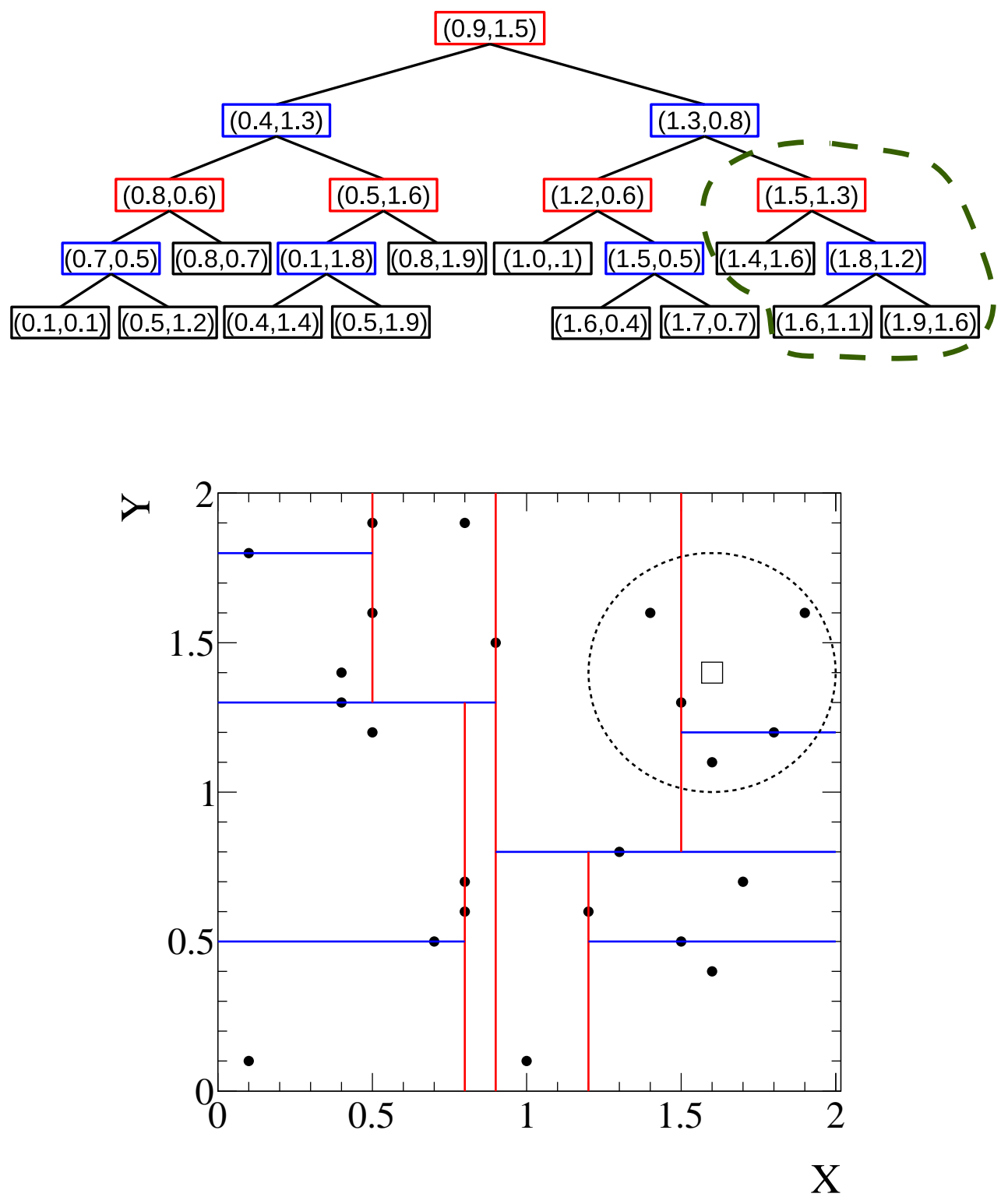

Figure A.3: An example of a k-D tree. Top: the binary tree is shown for data with two dimensions. The red and blue outlined boxes represent nodes which divide the feature space in "X" or "Y". The black outlined boxes represent "leaf" nodes, which are end-points in the k-D tree. Bottom: A 2-D plot of the points in the above $\mathrm{k}-\mathrm{D}$ tree. The red and blue lines represent the partitions made by each successive node. 
container of nodes would contain the nodes at the following positions: $(1.5,1.3),(1.8,1.2),(1.9,1.6),(1.3,0.8)$ and $(0.9,1.5)$. Note that these are not the nodes closest to the test point.

2. unwind the recursion, progressively moving to earlier nodes and check the unexplored branches,repeating the calculation in the previous step. In the specific case, the nodes at $(1.6,1.1)$ and $(1.4,1.5)$ will be added, and the nodes at $(1.3,0.8)$ and $(0.9,1.5)$ will be removed from the list.

The recursion stops when one reaches the root node again, or the unexplored branch only contains nodes further than the last node in the ordered container. In the specific example of Figure A.3, the node at $(1.3,0.8)$ is further than any of the nodes contained in the ordered container given by the points in the dotted circles.

\section{A.3 The Number of Nearest Neighbors and dimension- ality}

For the example shown in Figure A.3, the k-D tree algorithm only tested two points which were not in the final sample of events. In contrast, a brute-force exhaustive search would potentially check all eighteen points in the sample. The efficacy of the k-D tree method when compared to exhaustive searches increases with larger data samples and decreases with number of dimensions of the feature space. The time to find the nearest neighbor for a $\mathrm{k}$-d tree increases as

$$
\left.t_{w o r s t}=O\left(n \cdot N^{(} 1-\frac{1}{n}\right)\right)
$$

where $n$ is the number of dimensions and $N$ is the number of nodes, or objects in the feature space. In comparison, an exhaustive linear search method has a 
worst time which increases as $t_{\text {worst }}^{\text {linear }}=O(n N)$. Therefore, for high-dimension feature spaces, the k-D tree does not do significantly better than an exhaustive search.

The dimensionality of the feature space also has a direct effect on the effectiveness of the k-NN algorithm. The amount of data needed maintain a particular density increases proportionally with the volume of the feature space. Therefore, each new dimension requires an exponential increase in the amount of available training data. Without such increases in the training data, the approximation of the nearest-neighbor will begin to fail, as neighbors are not 'near' enough to approximate the test event well. This was one of the motivations to use two k-NN algorithms instead of one containing twice as many variables for the MINOS separation of charged current and neutral current events. This is demonstrated in Figure A.3. The area near test event A has one tenth the density of training events as the area near test event B. Therefore, for the same number of nearest neighbors as test event B, the search is wider for test event $\mathrm{A}$ and the ratio is affected by non-local fluctuations. For a number of nearest neighbors where test event $\mathrm{A}$ is not affected by non-local fluctuations, the binomial error on the nearest-neighbor search increases to a point where the value $\theta$ is no longer meaningful.

The density of training data in the feature space is especially relevant near the edges of the space. Because no training data exists outside some boundary, if a test event is close enough to the boundary such that the set of nearest-neighbors is heavily skewed off-center of the test-event location, then the approximation, $\theta$ will also be skewed inappropriately. Note that in higher dimensions, because of both the 


\section{A.4 Other Multivariate Techniques}

The k-NN technique was the preferred classification algorithm of the MINOS experiment because of its well documented effectiveness and because it had already been optimized for MINOS-like parameters. However, some thought was given to whether the $\mathrm{k}-\mathrm{NN}$ algorithm was indeed the best algorithm for separating charged current and neutral current events. Methods such as the linear-discriminant assume a Gaussian density of the data. This is not a feature of the MINOS and MINER $\nu \mathrm{A}$ data and therefore is not applicable. In fact, the distributions of the charged current and neutral current should not be assumed to conform to any particular equation ( because of detector uncertainties).

One method that in theory should be competitive with the k-NN method without unnecessarily increasing complexity is the Support Vector Machine (SVM) algorithm. Briefly, the SVM algorithm defines a hyperplane such that there is maximum separation between the two classes (the maximum-margin hyperplane). The classification of a test event will depend on which side of this hyperplane the event is on. The shortest vector to the hyperplane (including sign) can be used as a more variable selection parameter. The local hyperplane is supported by the vectors to the nearby training data. Therefore, both the SVM and k-NN obtain their power through the local density of the training data. However, the SVM algorithm is also sensitive to the position of the training data relative to the test point. Therefore, it has the potential to out-perform the k-NN.

Unfortunately, unlike the $\mathrm{k}-\mathrm{NN}$ whose training time is relatively short (consisting of just the time to construct the k-D tree), the SVM has a much longer and more complex training algorithm, which requires finding the maximum- 
distance hyperplane using every single training point. This made the SVM much more cumbersome to work with. Additionally, the SVM algorithm has many non-physical options. In particular, many different kernels can be used with the SVM (linear, polynomial, etc.) and each one should be viewed as a completely separate data-mining algorithm. Thus, the process of validating a SVM is much more difficult. Finally, preliminary results show no significant improvement in charged current/neutral current separation by using a standard SVM in MINOS. Therefore, the idea was not pursued further. The k-NN algorithm was found to have more power than any other standard method [106]. 


\title{
Appendix B
}

\section{Low-Energy events added by the CC/NCSeparator}

\begin{abstract}
Seven of the eight events regained below $2 \mathrm{GeV}$ by the new thresholds given in equation (6.2) compared to the threshold given in equation (6.1). Only one event has a significant shower. This shower is still significantly far from the track. The track energy for each event is relatively even, and all the tracks do not exhibit any kinking or other indicators of a mis-identified particle.
\end{abstract}

\section{B.1 ADC colored plots}

Note that the ADC colored plots, the color axis changes between plots. 

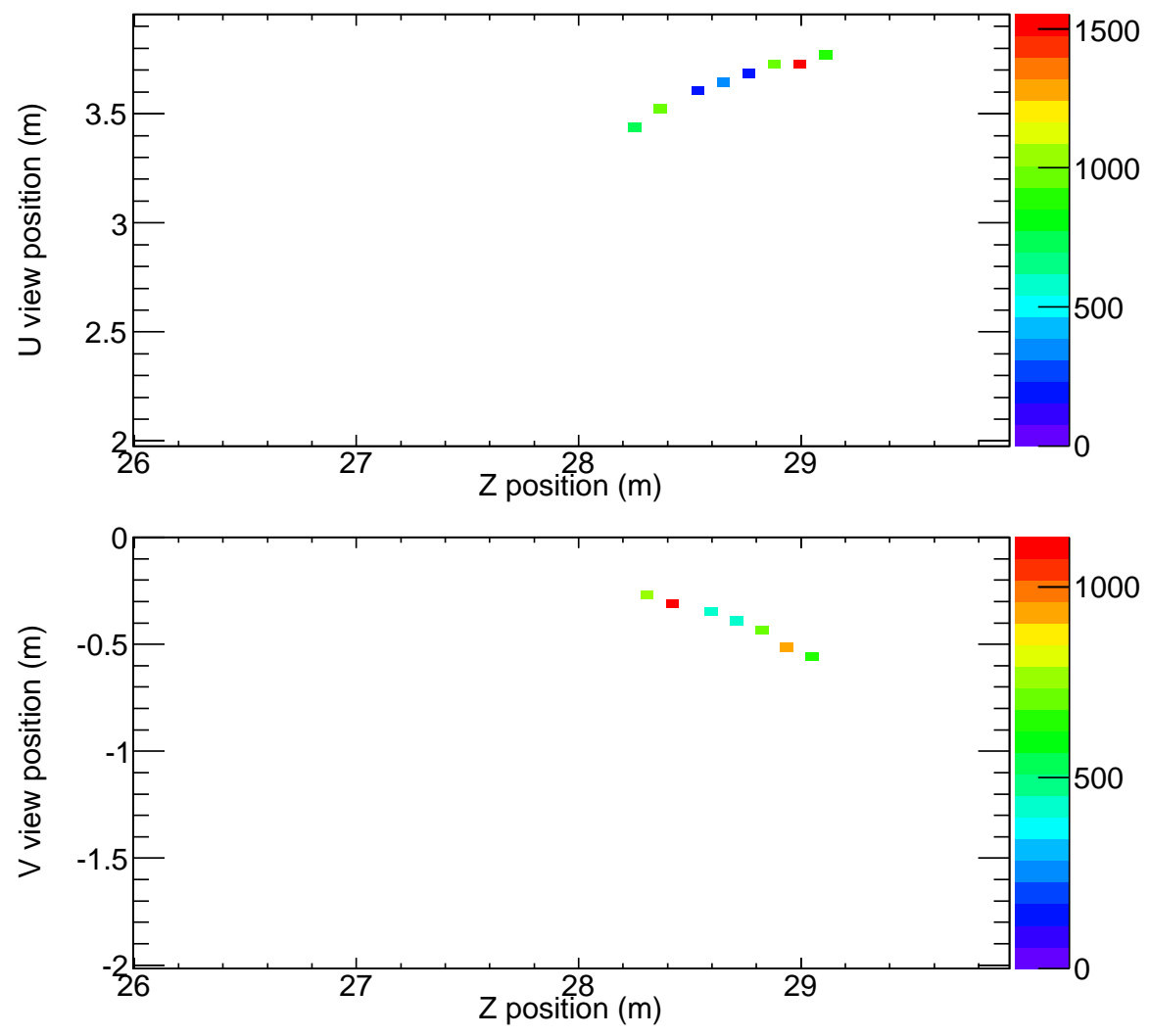

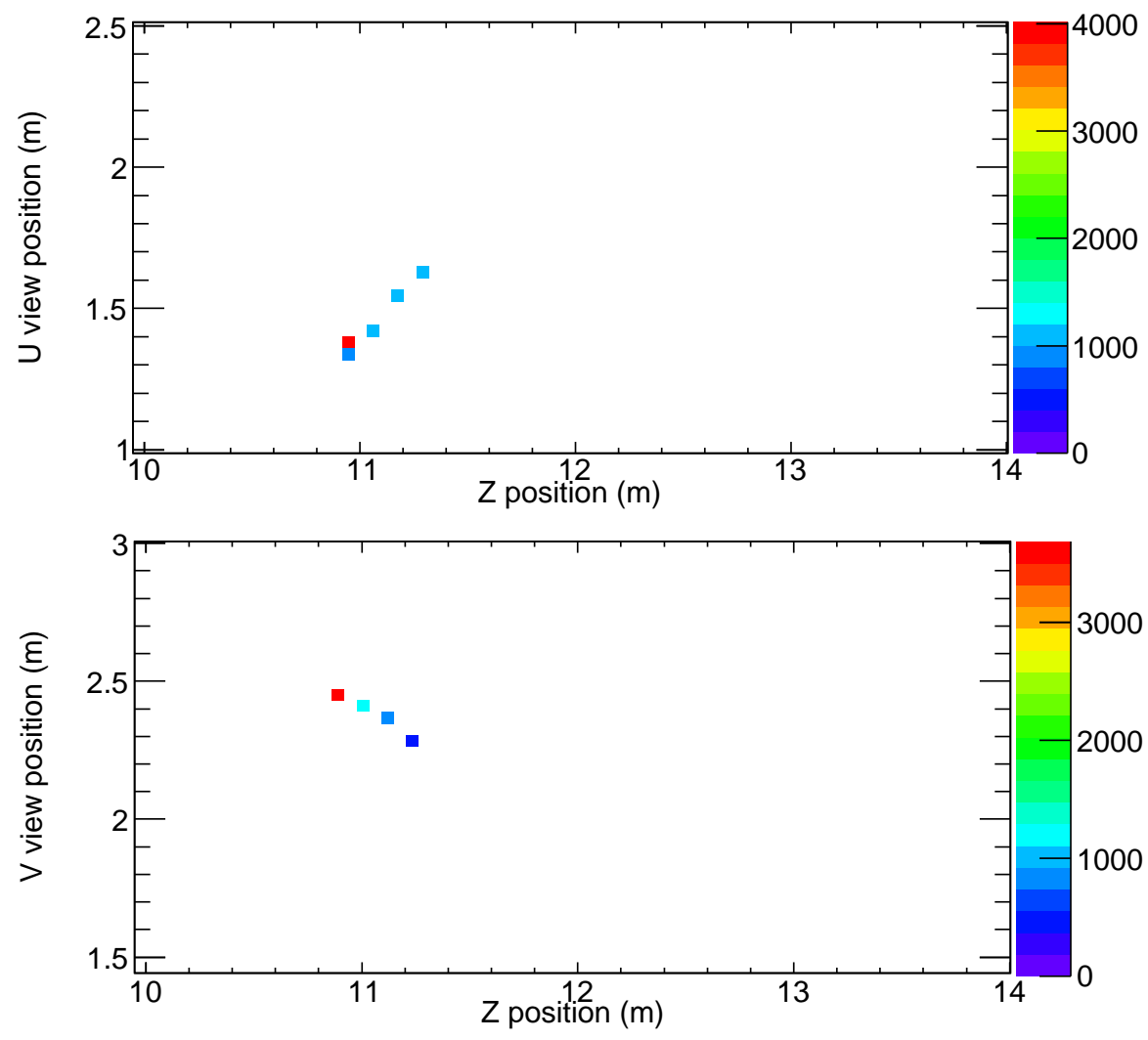

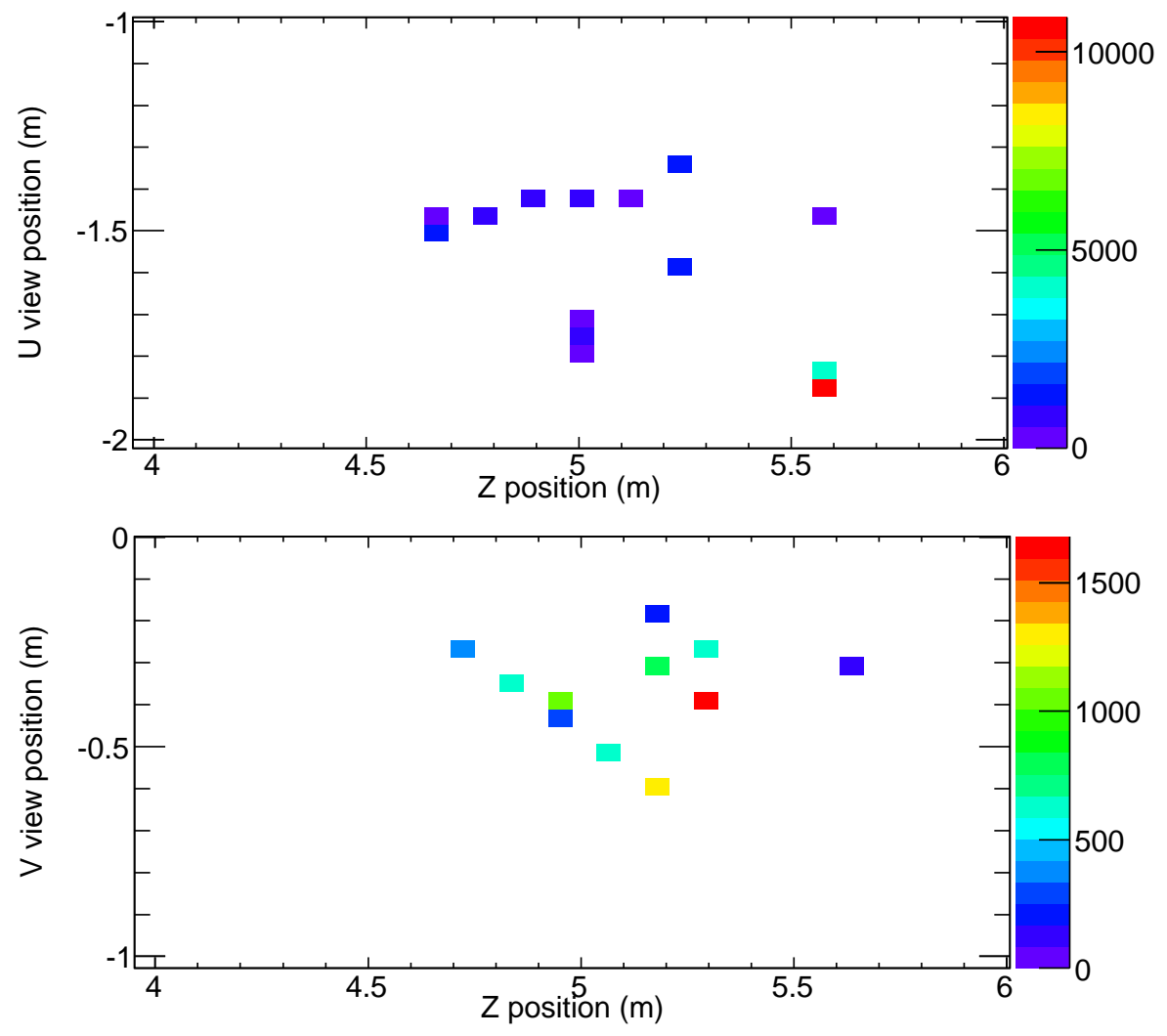

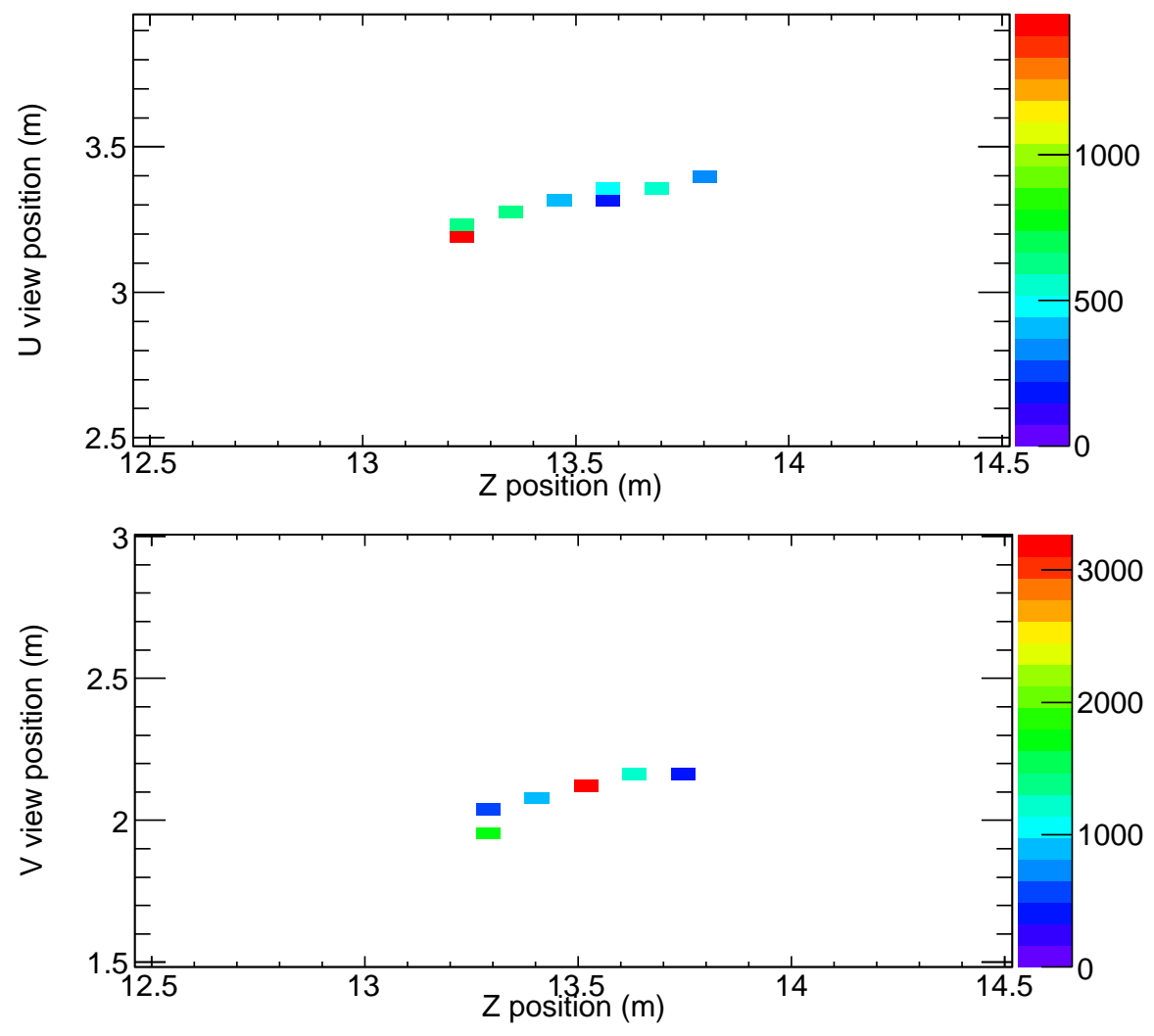

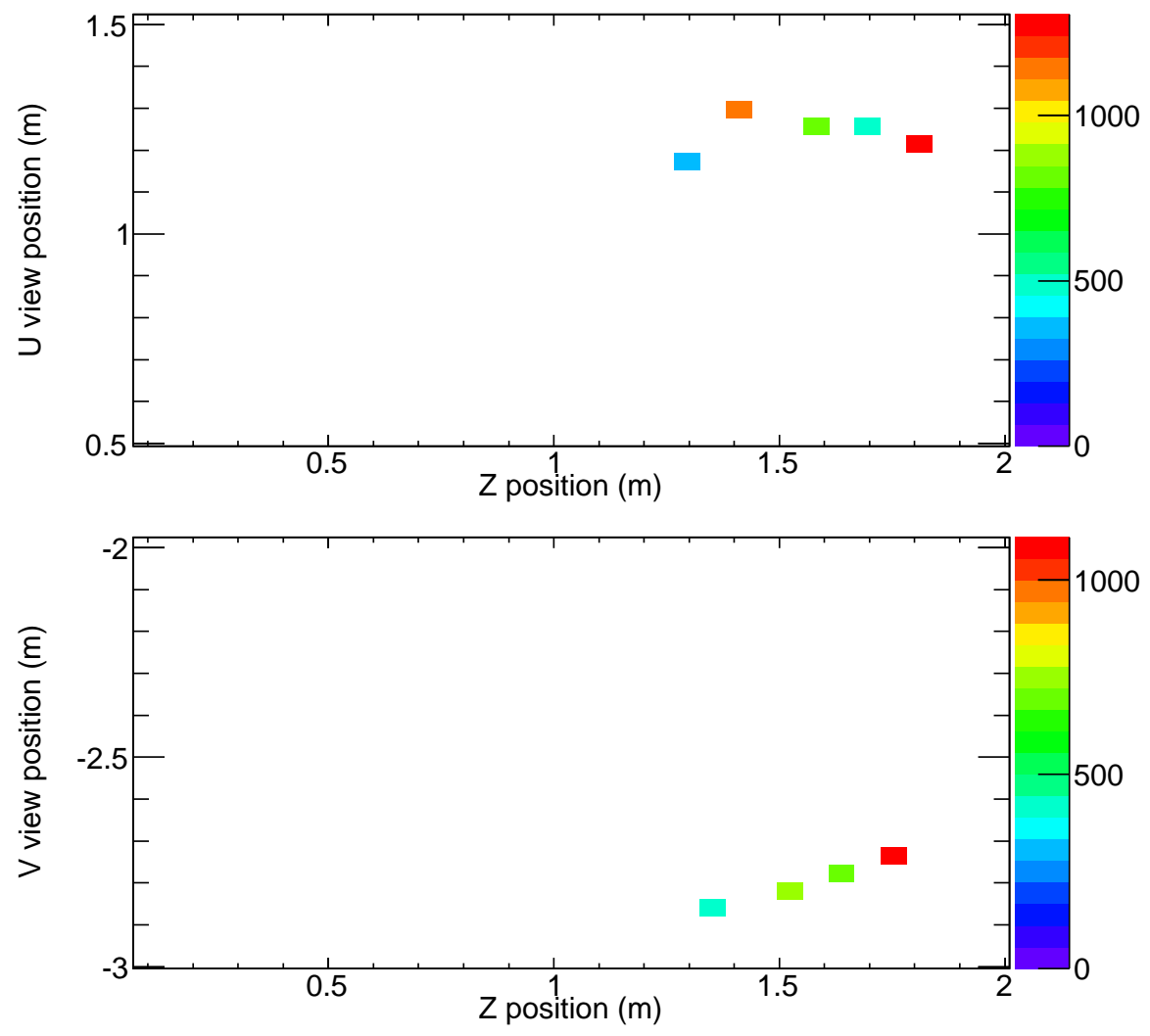

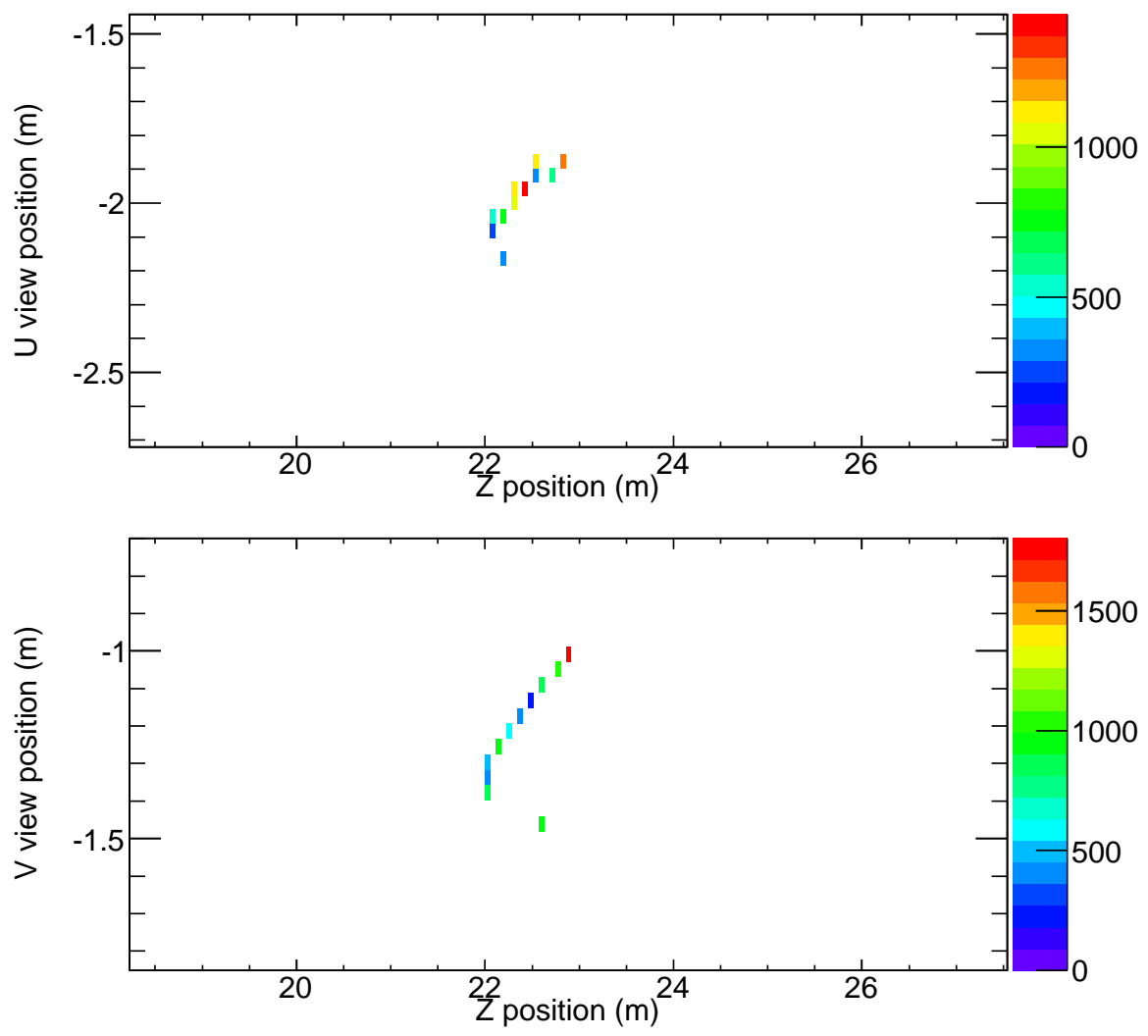

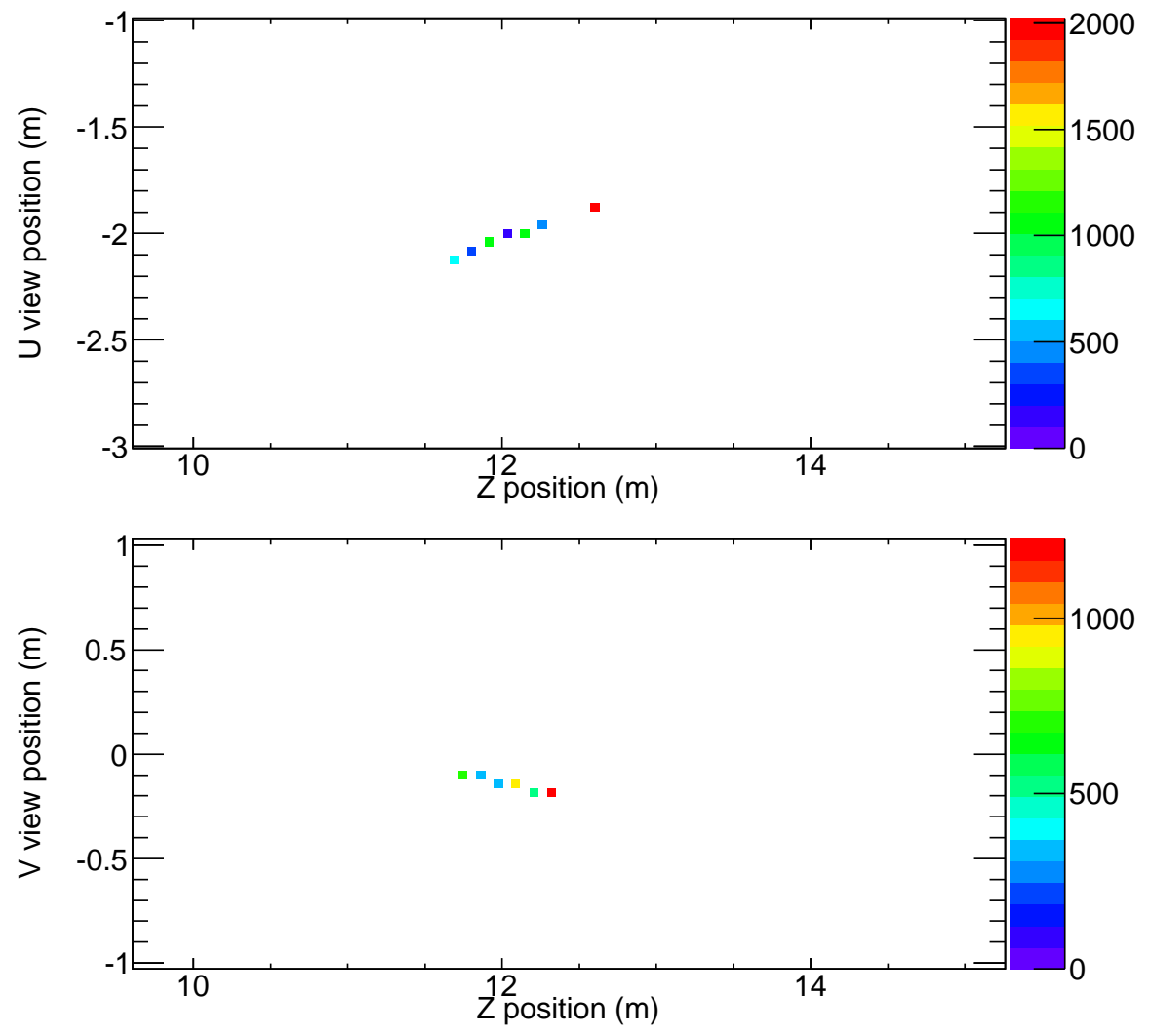

\section{B.2 Track and shower hit plots}

The grey regions in the track/shower colored plots represents antifiducial regions within the detector. These figures represent the same events and are in the same order as the last sections. The colors represnt (red) track and (green) shower hits. 

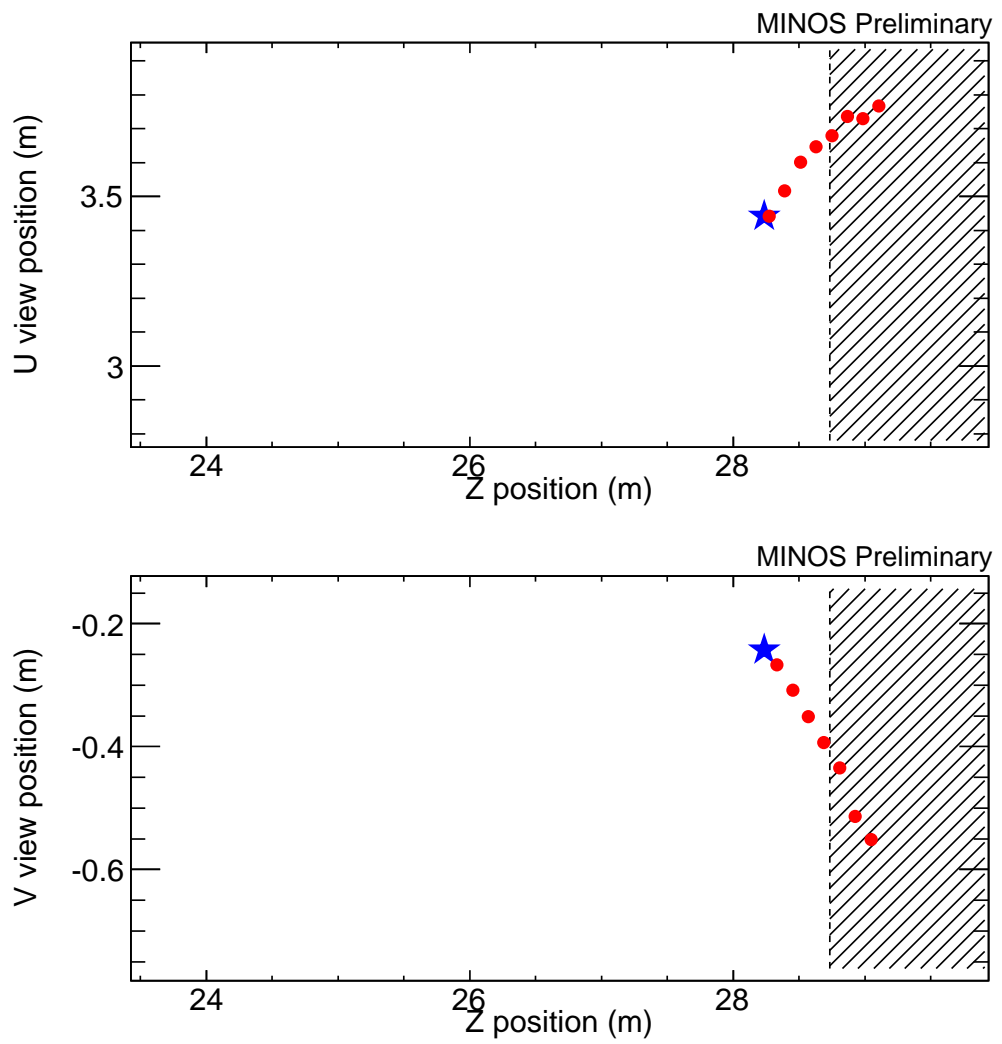

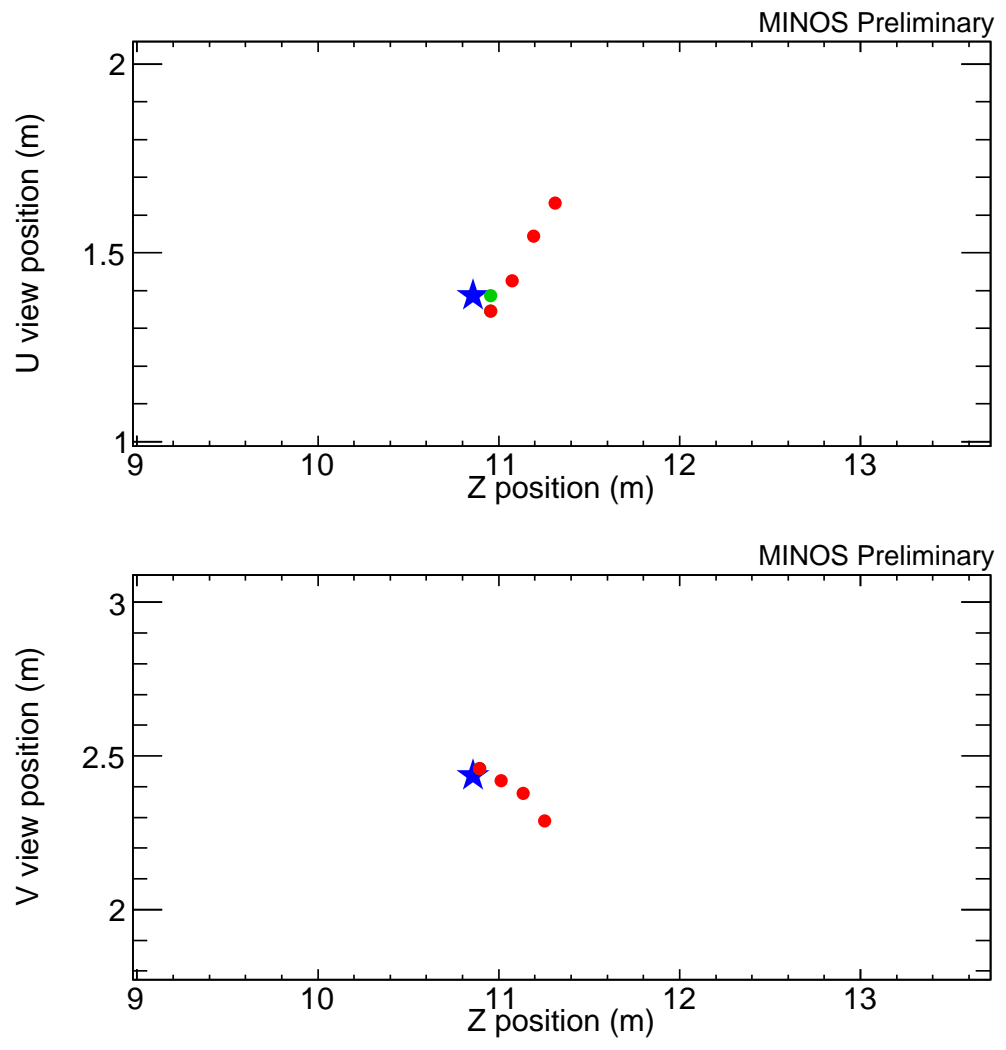

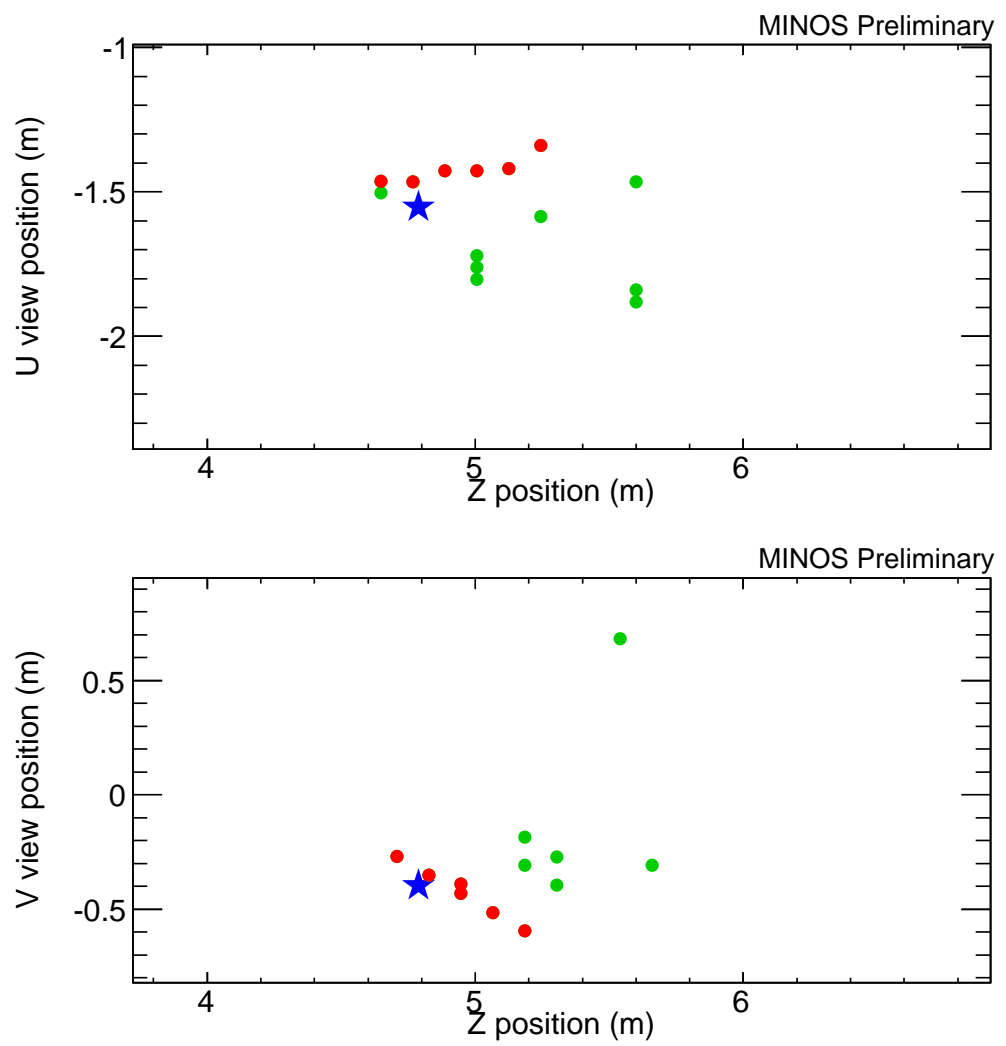

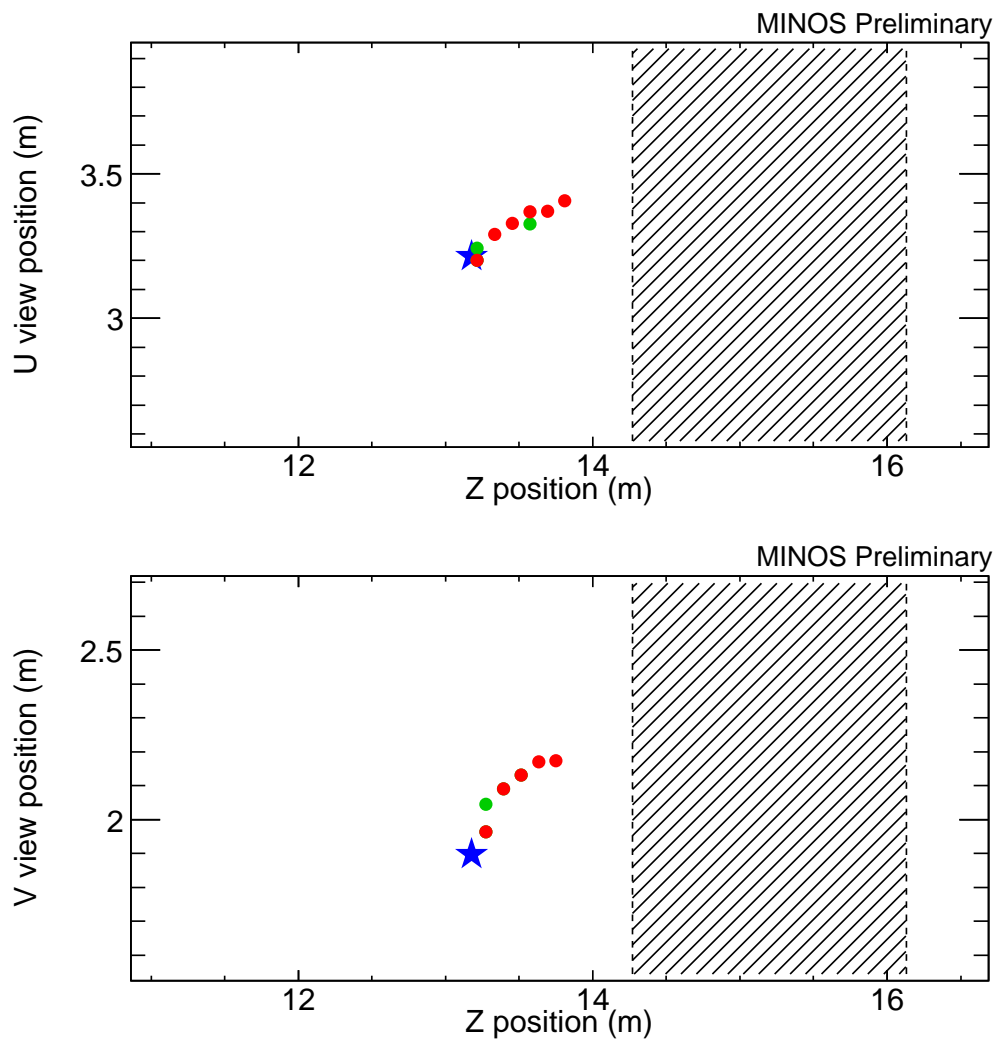

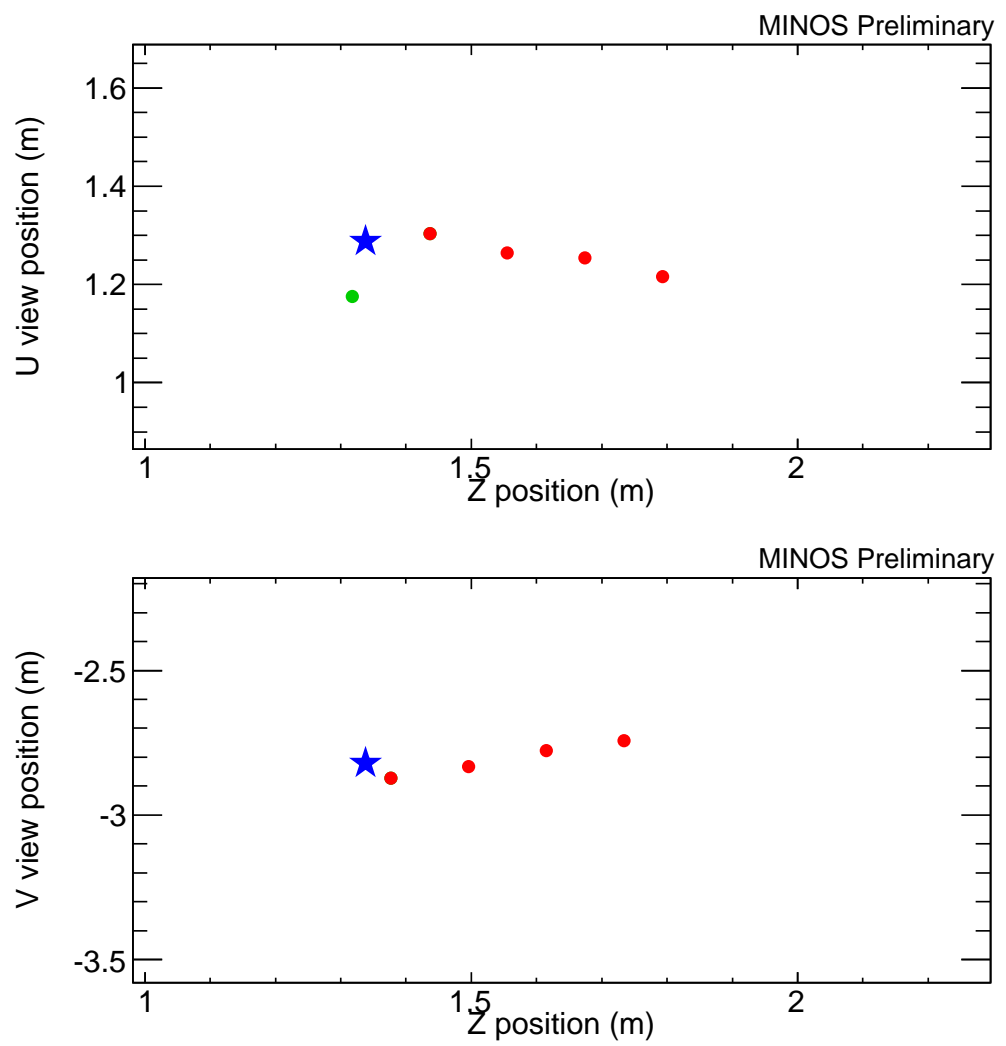

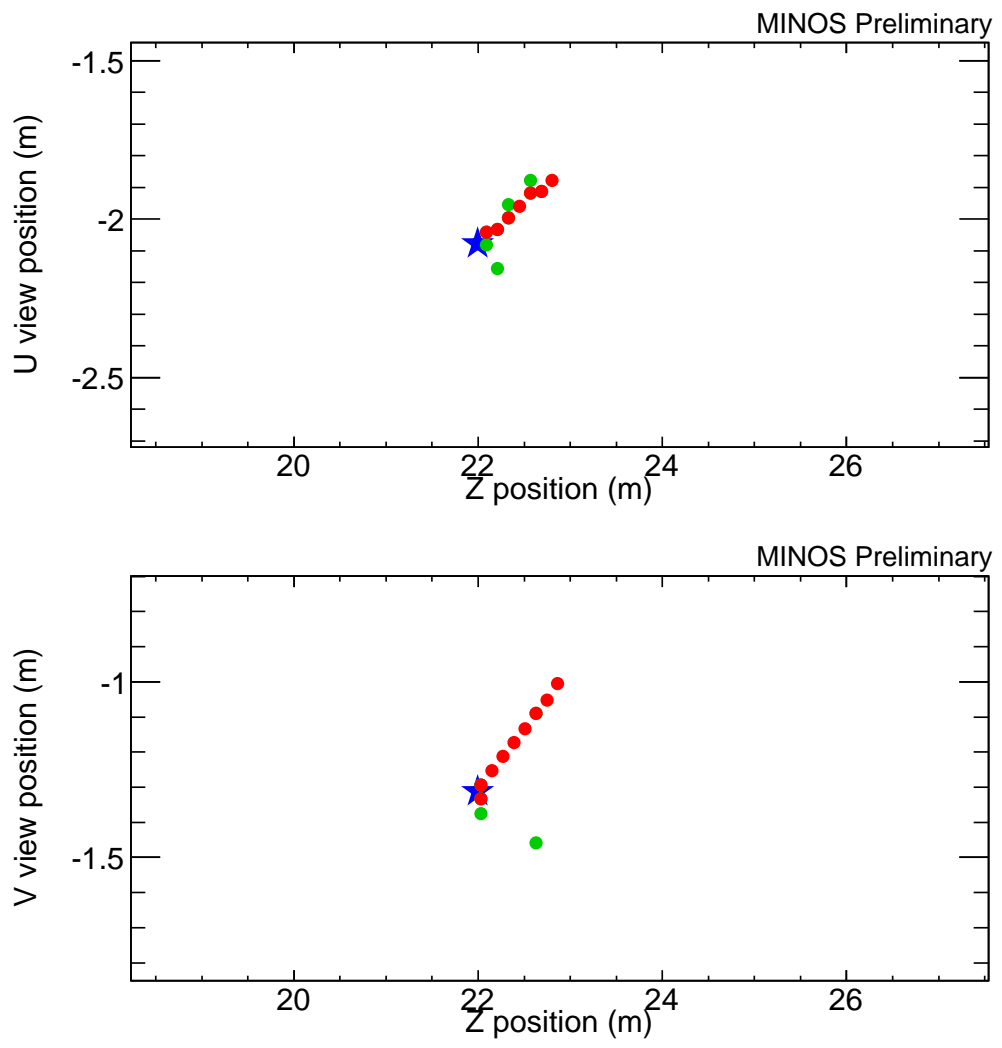

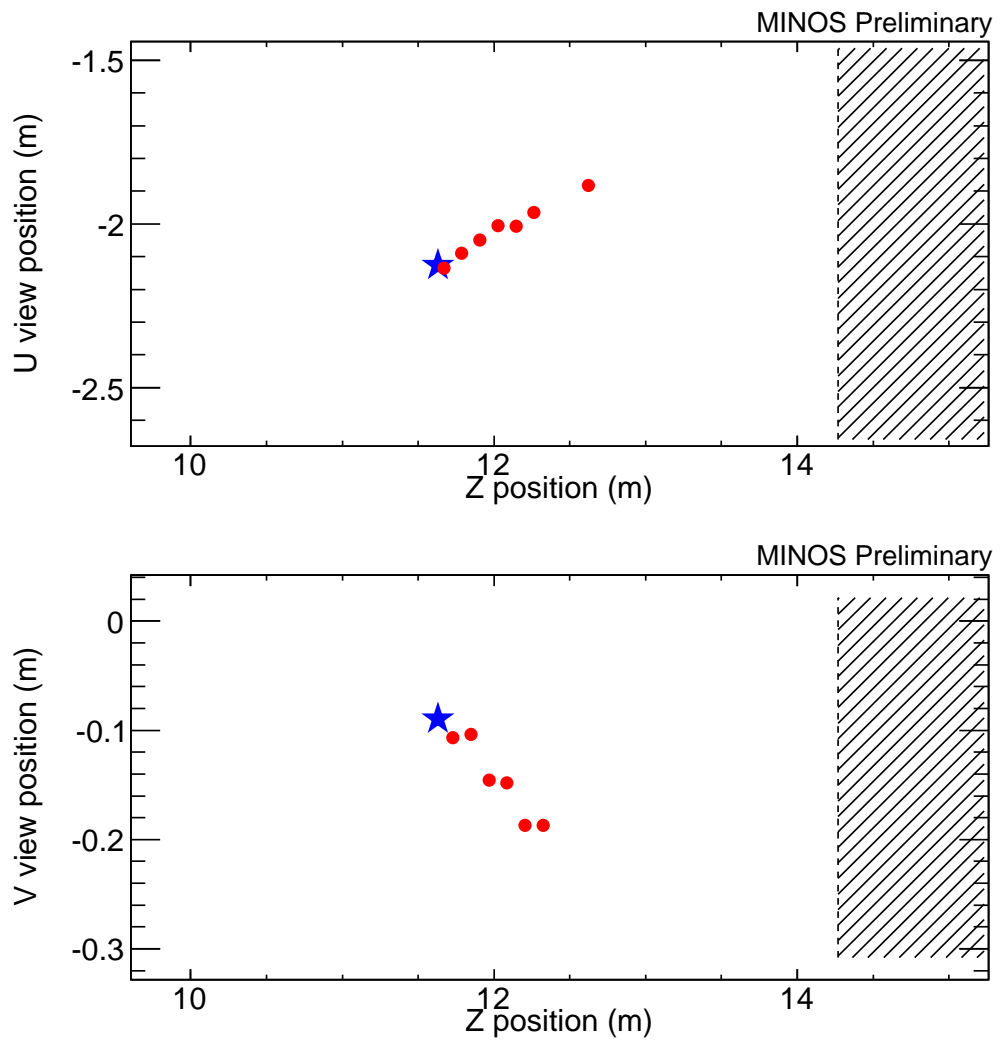


\section{Appendix C}

\section{The Neutral Current Background Study: Distributions from Daikon04/Dogwood1}

During the progress of these studies, the MINOS reconstruction and Monte Carlo versions were updated from 'Daikon04/Dogwood1' to 'Daikon07/ Dogwood3'. This presented MINOS with the unique opportunity to compare the distributions using two different Monte Carlo and reconstruction software releases. This appendix describes the earlier 'Daikon04/Dogwood1' version which was used for much of the studies which lead to the final systematic errors used in the 2010 MINOS $\nu_{\mu}$ disappearance analysis. The reconstruction and Monte Carlo upgrades had the effect of improving data and Monte Carlo agreement. This effect is small and the updated study, which is the focus of the previous two sections, had minimal differences to the 'Daikon04/Dogwood1' study. For completeness, a discussion of the 'Daikon04/Dogwood1' analysis is given here.

In Figure C.1, the muon-removed charged current and neutral current energy and inelasticity distributions are compared, normalized to unit area. These distributions are near identical to the distributions shown earlier in Figure 5.7.

Once again, the muon-removed charged current data and Monte Carlo for events which pass the 2010 MINOS $\nu_{\mu}$ analysis thresholds are plotted as a function of the inelasticity. The inelasticity distribution is given in Fig- 

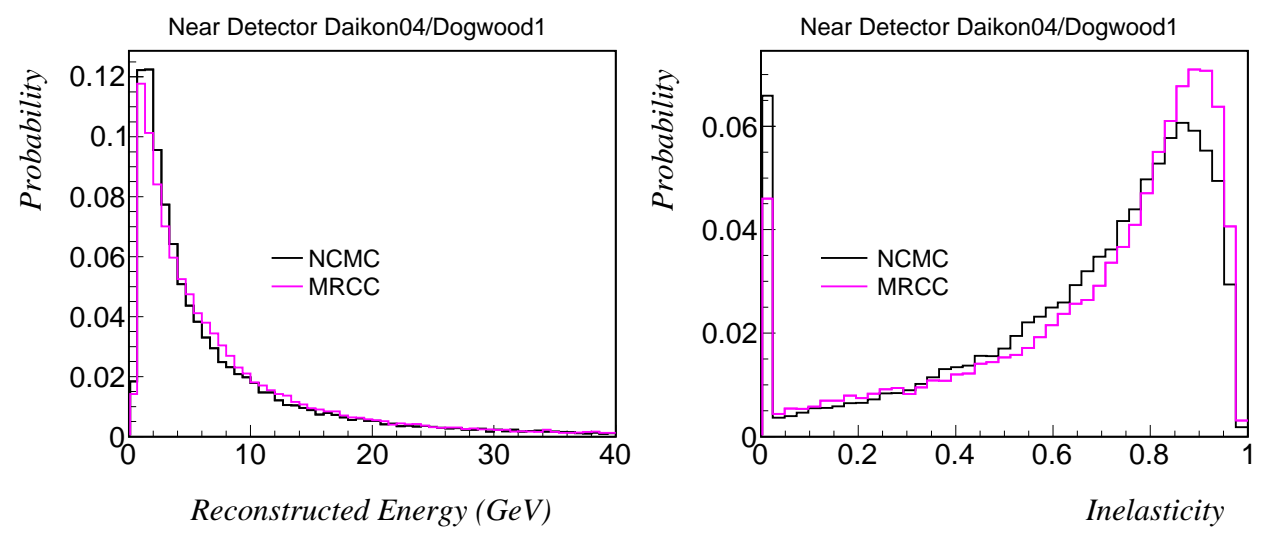

Figure C.1: Area normalized distributions of the neutral current and muonremoved charged current energy and inelasticity distributions

ure C.2 show a similar shape to those in using the update Monte Carlo and reconstruction.

Themuon-removed charged current data to Monte Carlo ratio is fitted to a fifth order polynomial. The result of this fit is the equation given in equation (C.1).

$$
\begin{aligned}
\Delta(\mathbf{y}) & =\frac{M R C C_{\text {data }}(\mathbf{y})}{M R C C_{\text {MonteCarlo }}(\mathbf{y})} \\
& =0.81-1.97 \mathbf{y}+15 \mathbf{y}^{2}-39.6 \mathbf{y}^{3}+46.39 \mathbf{y}^{4}-19.65 \mathbf{y}^{5}
\end{aligned}
$$

Note that this particular ratio is comparable to the grey points in Figure 5.10. This is because this particular fit was done with the same mixture of muonremoved charged current POT counting but original event SKZP reweighting. As was stated before, this scales all the points by the same amount and therefore does not affect the range of the muon-removed charged current data-Monte Carlo ratio. The range of muon-removed charged current data to Monte Carlo ratio is similar to the range shown previously in Figure 5.10.

Finally, the muon-removed charged current data to Monte Carlo ratio 

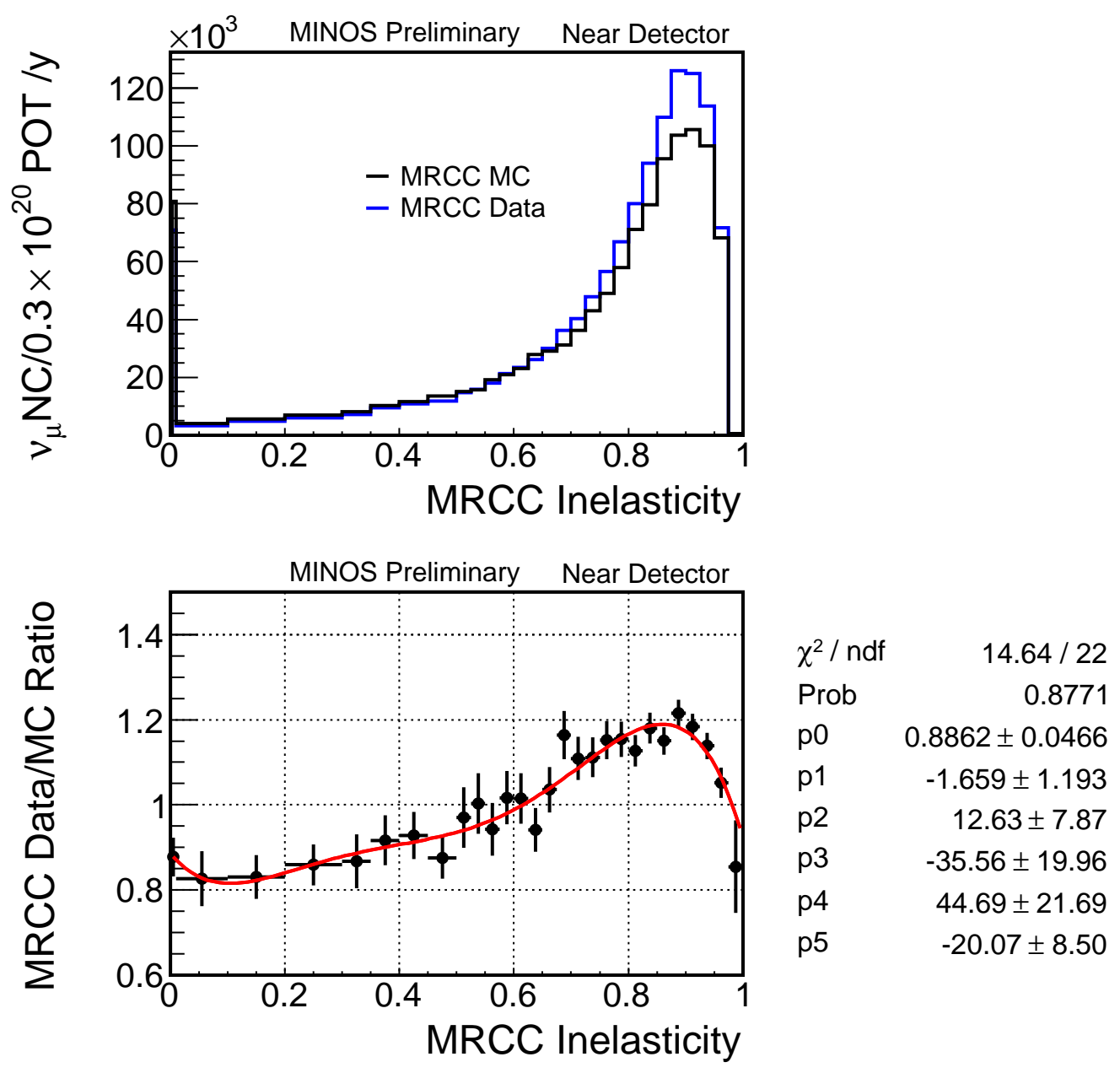

Figure C.2: The inelasticity distribution for Daikon04/Dogwood1 muonremoved charged current data and Monte Carlo events and the data to Monte Carlo ratio. 
is given for both the inelasticity and the energy. This is shown in Figure C.3. The histogram of the number of points of a particular data-Monte Carlo ratio value is not as Gaussian as the one shown in Figure 5.12, however, there is still a roughly $15 \%$ range in the values of the data/Monte Carlo ratio. The fit of

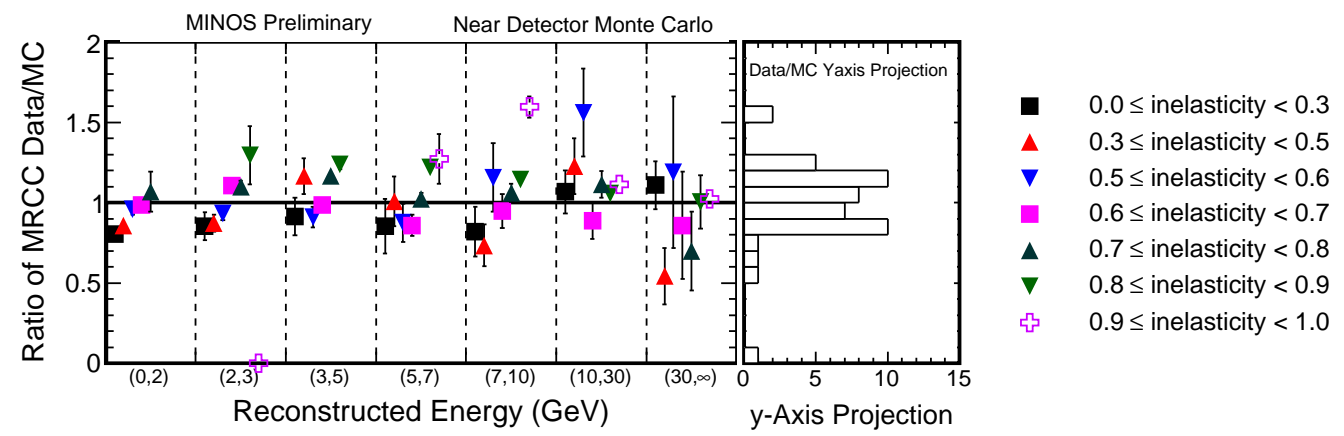

Figure C.3: The muon-removed charged current data/Monte Carlo ratio versus reconstructed energy and inelasticity for Daikon04, Dogwood3 Monte Carlo and reconstruction.

the muon-removed charged current data to Monte Carlo ratio with a constant value gives a fit value of

$$
\Delta=\frac{\text { MRCCdata }}{\text { MRCCMonteCarlo }}=1.02 \pm 0.15 .
$$

This confirms the roughly 15\% range of the muon-removed charged current data to Monte Carlo ratio.

The normalization study was also completed using 'Daikon04/Dogwood1' data and Monte Carlo. The $\mu \mathrm{ID}-\mathrm{B}$ distribution before and after scaling the Monte Carlo (with the charged current distribution as well as the neutral current distirbution scalable) to fit the data best is shown in Figure C.4. The complete results are shown in Table C. As one can see, varying the neutral current and charged current Monte Carlo together produces the largest deviation from the nominal scaling. The best fit between data and Monte Carlo 
is produced when the neutral current distribution is scaled down by roughly $11 \%$ and the charged current distribution is scaled up by $2 \%$. We use this largest deviation as the normlaization uncertainty in our final result. This is similar to what was done previously [74] by Marshall. Note that this supposes that the charged current distribution is also off by $2 \%$, even though other measurements of the charged current distributions constrain our charged current uncertainty to $1.6 \%$.

Alternatively, MINOS could also use the "charged current-Fixed" neutral current scaling of $94 \%$ as the error. This assumes that the charged current distribution is perfectly known, which is an underestimation of the neutral current error. The neutral current normalization in the Far Detector is fitted in a similar fashion (except the distribution is the reconstructed neutrino energy) as part of the MINOS $\nu_{\mu}$ disappearance measurement, and therefore, it is useless to apply such a correction to the neutral current Monte Carlo, as we did in the muon-removed charged current study to get the best fit to data and Monte Carlo. This weight also assumes that all differences between data and Monte Carlo are because of uncertainty in the normalization of the charged current and neutral current distributions. This is likely an exaggeration of the size of the normalization as a source of error. Therefore, this $11 \%$ fit discrepancy is considered a measurement of error rather than a possible scaling parameter. 


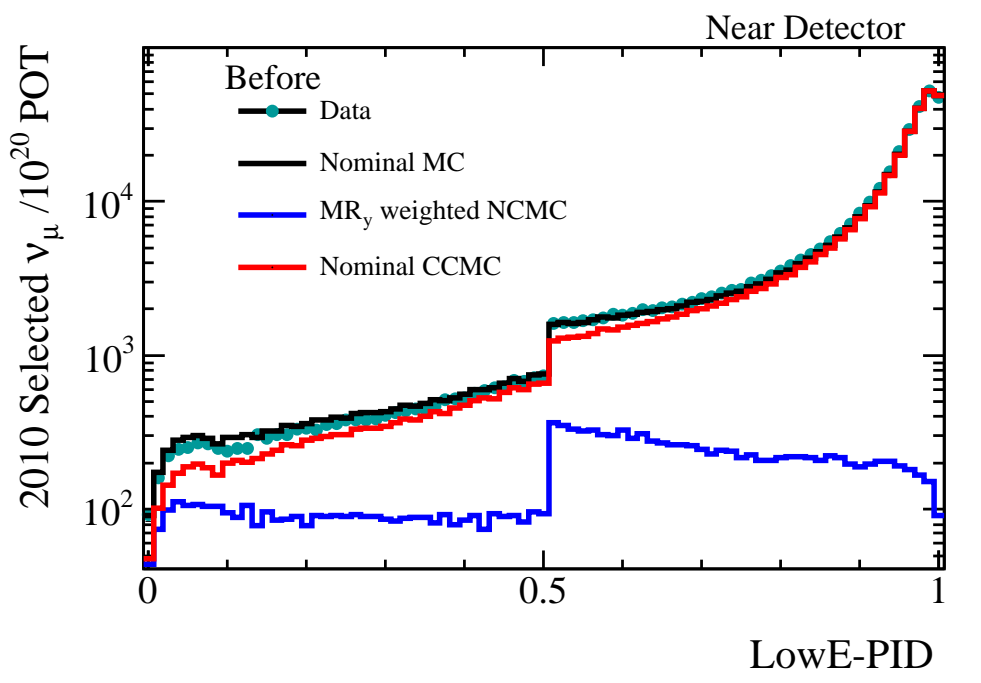

(a) Before

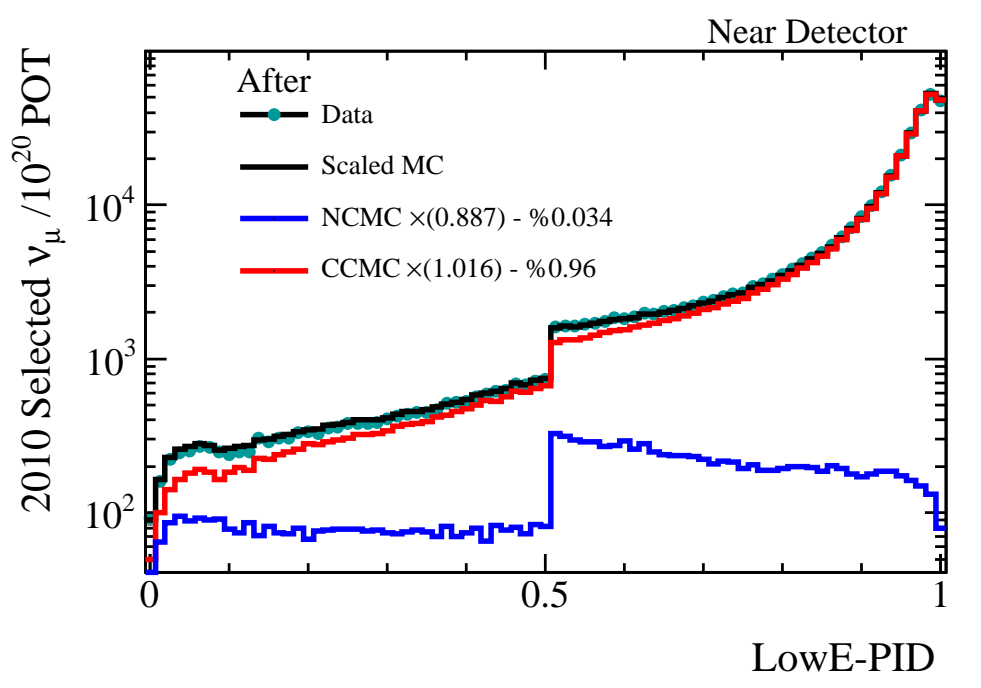

(b) After

Figure C.4: The normalization study using "Daikon04/Dogwood1" Monte Carlo and reconstruction. Here we show only the results for the normalization fitting while allowing the charged current distribution normalization to scale as well. These distributions are very similar to Figure 5.16. 


\begin{tabular}{|l|l|l|l|l|l|}
\cline { 2 - 5 } \multicolumn{1}{c|}{} & \multicolumn{2}{l|}{$\begin{array}{l}\text { Percentage of the Total } \\
\text { Distribution }\end{array}$} & \multicolumn{2}{l|}{$\begin{array}{l}\text { Original } \\
\text { scaled by }\end{array}$} & \multicolumn{2}{c|}{ distribution } \\
\cline { 2 - 6 } & $\begin{array}{l}\text { charged } \\
\text { current }\end{array}$ & $\begin{array}{l}\text { neutral cur- } \\
\text { rent }\end{array}$ & $\begin{array}{l}\text { charged } \\
\text { current }\end{array}$ & $\begin{array}{l}\text { neutral cur- } \\
\text { rent }\end{array}$ & $\chi^{2}$ \\
\hline Unfitted & $94.0 \%$ & $4.6 \%$ & 1.0 & 1.0 & NA \\
\hline Fixed & $94.0 \%$ & $4.48 \pm 0.13 \%$ & 1.0 & $96.4 \pm 4.4 \%$ & 3.27 \\
\hline Unfixed & $96.0 \pm 0.2 \%$ & $3.96 \pm 0.13 \%$ & $1.02 \pm 0.002$ & $0.89 \pm 0.03$ & 2.47 \\
\hline
\end{tabular}

Table C.1: Normalization values for neutral current and charged current Monte Carlo before and after re-weighting. The columns represent (1) the name of the fit,the percentage of (2) charged current and (3) neutral current in the total Monte Carlo, the value by which the nominal (4) charged current and (5) neutral current Monte Carlo distributions are scaled, and (6) the $\chi^{2}$ value of the fit. The rows represent (A) the "Unfitted" original values of the charged current and neutral current Monte Carlo. In this row we see the nominal amount of charged current, and neutral current Monte Carlo. Because there is no fit, the $\chi^{2}$ is not found. (B) the "charged current Fixed" fixed of the charged current and neutral current Monte Carlo. In this row, the charged current distribution is held fixed and only the neutral current Monte Carlo varies. (note that the scale factor for the charged current distribution is 1.0) and $(\mathrm{C})$ the "charged current Unfixed" fit of the charged current and neutral current Monte Carlo. Here both the charged current and neutral current Monte Carlo distributions are allowed to vary (with a range in the charged current direction of $\pm 1.6 \%$ ). the charged current normalization error). This is performed with the older Daikon04 Monte Carlo and Dogwood1 reconstuction. 


\section{Bibliography}

[1] P. Fisher, B. Kayser, and K. S. McFarland, "Neutrino mass and oscillation," Ann.Rev.Nucl.Part.Sci., vol. 49, pp. 481-528, 1999.

[2] R. Davis, D. S. Harmer, and K. C. Hoffman, "Search for neutrinos from the sun," Phys. Rev. Lett., vol. 20, pp. 1205-1209, May 1968.

[3] Z. Maki, M. Nakagawa, and S. Sakata, "Remarks on the unified model of elemntary particles," Progress of Theoretical Physics, vol. 28, pp. 870880, 1962.

[4] B. Pontecorvo, "Neutrino experiments and the problem of conservation of leptonic charge," Sov. Phys. Journal of Experimental and Thoretical Physics, vol. 53, p. 1717, 1967.

[5] J. N. Bahcall, "Solar neutrino cross sections and nuclear beta decay," Phys. Rev., vol. 135, pp. B137-B146, Jul 1964.

[6] J. Goldstone, A. Salam, and S. Weinberg, "Broken symmetries," Phys. Rev., vol. 127, pp. 965-970, Aug 1962.

[7] S. Weinberg, "A model of leptons," Phys. Rev. Lett., vol. 19, pp. 12641266, Nov 1967.

[8] S. Glashow, "Partial Symmetries of Weak Interactions," Nucl.Phys., vol. 22 , pp. 579-588, 1961. 
[9] B. Pontecorvo, "Mesonium and antimesonum," Sov. Phys. Journal of Experimental and Theoretical Physics, vol. 6, p. 429, 1958.

[10] B. Pontecorvo, "Inverse beta decay processes and the question of leptonic charge conservation," Sov. Phys. Journal of Experimental and Thoretical Physics, vol. 7, pp. 172-173, 1958.

[11] V. Gribov and B. Pontecorvo, "Neutrino astronomy and lepton charge," Physics Letters B., vol. 28, p. 493, 1969.

[12] R. E. Shrock and L.-L. Wang, "A New Generalized Cabibbo FIT and Application to Quark Mixing Angles in the Sequential Weinberg-Salam Model," Phys.Rev.Lett., vol. 41, p. 1692, 1978.

[13] H. J. Lipkin, "Quantum theory of neutrino oscillations for pedestrians: Simple answers to confusing questions," Phys.Lett., vol. B642, pp. 366$371,2006$.

[14] B. Cleveland, T. Daily, R. Davis Jr., J. R. Distel, L. Kenneth, C. Lee, and W. P. S., "Measurement of the solar electron neutrino flux with the homestake chlorine detector," ApJ, vol. 496, pp. 505-524, 1998.

[15] A. Bandyopadhyay, S. Choubey, S. Goswami, and D. Roy, "Implications of the first neutral current data from sno for solar neutrino oscillation," Phys.Lett., vol. B540, pp. 14-19, 2002.

[16] S. Collaboration, "Electron energy spectra, fluxes, and day0night assymetries of ${ }^{8} b$ solar neutrinos from measurements with nacl disolved in the eavy-water detector at the sudbury neutrino observatory," Physical Review C, vol. 72, p. 055502, 2005. 
[17] T. S.-K. Collaboration, "Atmospheric neutrino oscillation analysis with subleading effects in super-kamiokande i, ii, and iii," Physical Review D, vol. 81, p. 092004, 2010.

[18] E. Aliu et al., "Evidence for muon neutrino oscillation in an acceleratorbased experiment," Phys.Rev.Lett., vol. 94, p. 081802, 2005.

[19] K. Hirata et al., "Experimental study of the atmospheric neutrino flux," Phys.Lett., vol. B205, p. 416, 1988.

[20] Y. Fukuda et al., "Evidence for oscillation of atmospheric neutrinos," Phys. Rev. Lett., vol. 81, pp. 1562-1567, Aug 1998.

[21] P. Adamson et al., "Measurement of the neutrino mass splitting and flavor mixing by minos," Phys. Rev. Lett., vol. 106, p. 181801, May 2011.

[22] T. T. Collaboration, "Indication of electron neutrino appearance from an accelerator-produced off-axis muon neutrino beam," Phys. Rev. Lett., vol. 107, p. 041801, Jul 2011.

[23] A. Aguilar et al., "Evidence for neutrino oscillations from the observation of anti-nu/e appearance in a anti-nu/mu beam," Phys. Rev., vol. D64, p. 112007, 2001.

[24] A. A. Aguilar-Arevalo et al., "Search for electron neutrino appearance at the $\delta m^{2} \sim 1 \mathrm{ev}^{2}$ scale," Phys. Rev. Lett., vol. 98, p. 231801, Jun 2007.

[25] A. A. Aguilar-Arevalo et al., "Event excess in the miniboone search for $\overline{\nu_{\mu}} \rightarrow \overline{\nu_{e}}$ oscillations," Phys. Rev. Lett., vol. 105, p. 181801, Oct 2010. 
[26] M. Goldhaber, L. Grodzins, and A. Sunyar, "Helicity of neutrinos," Phys.Rev., vol. 109, pp. 1015-1017, 1958.

[27] A. Abada, C. Biggio, F. Bonnet, M. B. Gavela, and T. Hambye, " $\mu \rightarrow$ $e^{+} \gamma$ and $\tau \rightarrow l+\gamma$ decays in the fermion triplet seesaw model," Phys. Rev. D, vol. 78, p. 033007, Aug 2008.

[28] A. Goobar, S. Hannestad, E. Mortsell, and H. Tu, "The neutrino mass bound from wmap 3 year data, the baryon acoustic peak, the snls supernovae and the lyman- $\alpha$ forest," JCAP, vol. 0606, p. 019, 2006.

[29] W. Rodejohann, "Neutrino-less double beta decay and particle physics," Int.J.Mod.Phys., vol. E20, pp. 1833-1930, 2011.

[30] H. Fritzsch and P. Minkowski, "Parity conserving neutral currents and righthanded neutrinos," Nucl.Phys., vol. B103, p. 61, 1976.

[31] J. F. Beacom and N. F. Bell, "Do solar neutrinos decay?," Phys.Rev., vol. D65, p. 113009, 2002.

[32] E. Lisi, A. Marrone, and D. Montanino, "Probing possible decoherence effects in atmospheric neutrino oscillations," Phys.Rev.Lett., vol. 85, pp. 1166-1169, 2000.

[33] P. Adamson et al., "A study of muon neutrino disappearance using the fermilab main injector neutrino beam," Phys. Rev. D77, vol. 77, p. $072002,2008$.

[34] D. H. Perkins, Introduction to High Energy Physics. Addison Wesley, 1987. 
[35] P. D. Group, "Nuclear and particle physics," Journal of Physics G, vol. $37,2010$.

[36] P. Lipari, M. Lusignoli, and F. Sartogo, "The neutrino cross section and upward going muons," Phys. Rev. Lett., vol. 74, pp. 4384-4387, May 1995.

[37] C. Llewellyn Smith, "Neutrino reactions at accelerator energies," Physical Reports, vol. 5, pp. 261-379, 1972.

[38] D. Rein and L. Sehgal, "Neutrino-exitation of baryon resonances and single pion production," Annals of Physics, vol. 113, pp. 79-143, 1981.

[39] P. Lipari, "Neutrino oscillation studies and the neutrino cross section," Nuclear Physics B - Proceedings Supplements, vol. 112, no. 1-3, pp. 274 $-287,2002$.

[40] A. Bodek and U. Yang, "Modeling deep inelastic cross sections in the few gev region," Nuclear Physics B - Proceedings Supplements, vol. 112, no. 1-3, pp. $70-76,2002$.

[41] O. Benhar and D. Meloni, "Total neutrino and antineutrino nuclear cross-sections around 1-GeV," Nucl.Phys., vol. A789, pp. 379-402, 2007.

[42] P. Bosetti et al., "Analysis of nucleon structure functions in cern bubble chamber neutrino experiments," Nucl.Phys., vol. B142, pp. 1-28, 1978.

[43] A. Bodek and J. Ritchie, "Fermi Motion Effects in Deep Inelastic Lepton Scattering from Nuclear Targets," Phys.Rev., vol. D23, p. 1070, 1981. 
[44] C. Andreopoulos, A. Bell, D. Bhattacharya, F. Cavanna, J. Dobson, et al., "The GENIE Neutrino Monte Carlo Generator," Nucl.Instrum.Meth., vol. A614, pp. 87-104, 2010.

[45] D. Zieminska et al., "Charged-particle multiplicity distributions in $\nu n$ and nup charged-current interactions," Phys. Rev. D, vol. 27, pp. 4757, Jan 1983.

[46] S. Barlag et al., "CHARGED HADRON MULTIPLICITIES IN HIGHENERGY anti-muon neutrino n AND anti-muon neutrino p INTERACTIONS," Z.Phys., vol. C11, p. 283, 1982.

[47] K. Anderson et al., "The numi facility technical design report." FERMILABDESIGN-1998-01, 1998.

[48] Z. Pavlovic, Observation of Disapperance of Muon Neutrinos in the NuMI Beam. PhD thesis, University of Texas at Austin, 2008.

[49] R. M. Zwaska, Accelerator Systems and Instrumentation for the NuMI Neutrino Beam. PhD thesis, The University of Texas at Austin, 2005.

[50] S. Kopp, "Accelerator neutrino beams," Physics Reports, vol. 429, pp. 101159, 2007.

[51] L. Loiacono, Measurement of the Muon Neutrino Inclusive Charged Current Cross Section on Iron Using the MINOS Detector. PhD thesis, The University of Texas at Austin, 2010.

[52] J. R. A. Fasso, A. Ferrari and P. Sala, "Fluka: a multi-particle transport code.." CERN , INFN/TC 05/11, SLAC-R-773, 2005. 
[53] R. Brun, R. Hagelberg, M. Hansroul, and J. Lassalle, "GEANT: SIMULATION PROGRAM FOR PARTICLE PHYSICS EXPERIMENTS. USER GUIDE AND REFERENCE MANUAL," 1978. Revised Version.

[54] S. Kopp, Z. Pavlovic, and P. Vahle, "Skzp2: Update on fitting the beam mc to the nd data." MINOS-DOC 1650, Nov 2006.

[55] M. Dorman, "Beam fit position paper." MINOS-DOC 7146-v1, 2010.

[56] C. Alt et al., "Inclusive production of charged pions in $\mathrm{p}+\mathrm{C}$ collisions at $158-\mathrm{GeV} / \mathrm{c}$ beam momentum," Eur.Phys.J., vol. C49, pp. 897-917, 2007.

[57] H. Gallagher, "The neugen neutrino event generator," Nuclear Physics B - Proceedings Supplements, vol. 112, pp. 188-194, 2002.

[58] S. J. Coleman, A measurement of neutrino oscillations with muon neutrinos in the MINOS experiment. $\mathrm{PhD}$ thesis, The College of William and Mary, 2011.

[59] W. Leo, Techniques fo Nuclear and Particle Physics Experiements. Springer, 1994.

[60] S. Eidelman et al., "Review of particle physics. particle data group," Phys.Lett., vol. B592, p. 1, 2004. The Review of Particle Physics.

[61] R. Brun and F. Rademakers, "Root - an object oriented data analysis framework," Nucl. Inst. \&3 Meth. in Phys. Res. A, vol. 389, pp. 81-86, 1997. See also http://root.cern.ch/. 
[62] S. Moser, W. Harder, C. Hurlbut, and M. Kusner, "Principles and practice of plastic scintillator design," Radiation Physics and Chemistry, vol. 41, no. 1-2, pp. $31-36,1993$.

[63] P. Adamson et al., "The minos scintillator calorimeter system," IEEE Transactions on Nuclear Science, vol. 49(3), pp. 861-863, 2002.

[64] J. Hartnell, Measurement of the Calorimetric Energy Scale in MINOS. $\mathrm{PhD}$ thesis, Oxford, 2005.

[65] A. Marino, "A measurement of the density of minos scintillator strips." MINOS-DOC 2080, July 2006.

[66] G. Maps", "Numi beamline," October 2011.

[67] D. Michael et al., "The magnetized steel and scintillator calorimeters of the minos experiment," Nuclear Instruments and Methods in Physics Research A, vol. 596, pp. 190-228, 2008.

[68] T. M. Experiment, "The MINER $\nu$ A technical design report." This file is located at minerva-docdb.fnal.gov/0007/000700/028/TDR_v1.3.pdf.

[69] R. Ospanov, A measurement of muon neutrino disappearance with the MINOS detectors and NuMI beam. PhD thesis, University of Texas at Austin, 2008.

[70] A. Blake, "Near detector data/mc plots for cc analysis." Minos-DOC 7660-v1, 2010.

[71] A. Mann et al., "'bottom-fishing for qe-like events below the oscillation minimum - open season" and "methodology of hadron/muon separation at low momenta"." MINOS-DOC 3333-v1 and Minos-DOC 3214-v2. 
[72] Low-energy neutrino cross-sections: Comparison of various Monte Carlo predictions to experimental data, 2003. arXiv:hep-ex/0312061v1.

[73] J. S. Marshall, A study of muon neutrino disapperaance with the MINOS detector and the NuMI neutrino beam. PhD thesis, Fitzwilliam College, University of Cambridge, 2003.

[74] J. S. Marshall, "A data-driven correction to the me nc background." MINOS-DOC 3307-v1, June 2007.

[75] C. Howcroft, "Muon removal." MINOS-DOC 1333, 2005.

[76] T. Yang, "A revisit of the muon removal algorithm." MINOS-DOC 3973 (presentation), January 2008.

[77] T. Yang, A study of muon neutrino to electron neutrino oscillations in the MINOS experiment. PhD thesis, Stanford University, 2009.

[78] P. Adamson et al., "New constraints on muon-neutrino to electronneutrino transitions in minos," Phys.Rev., vol. D82, p. 051102, 2010.

[79] P. Adamson et al., "Search for muon-neutrino to electron-neutrino transitions in minos," Phys. Rev. Lett., vol. 103, p. 261802, Dec 2009.

[80] J. Boehm, "Measuring $\nu_{e}$ cc selection efficiency and systematic error using the muon removal with me electron addition process." MINOSDOC 5385-v4, January 2009.

[81] C. Backhouse, Measuring neutrino oscillation parameters using $\nu_{\mu}$ disapperance in MINOS. PhD thesis, Oxford, 2011. 
[82] D. Krakauer, R. Talaga, R. Allen, H. Chen, R. Hausammann, et al., "Direct experimental lower bound on the radiative lifetime of the muonneutrino," Phys.Rev., vol. D44, pp. 6-9, 1991.

[83] P. Adamson et al., "Measurement of neutrino oscillations with the minos detectors in the numi beam," Phys. Rev. Lett., vol. 101, p. 131802, Sep 2008.

[84] Y. Ashie et al., "Evidence for an oscillatory signature in atmospheric neutrino oscillation," Phys.Rev.Lett., vol. 93, p. 101801, 2004.

[85] M. H. Ahn et al., "Measurement of neutrino oscillation by the k2k experiment," Phys. Rev. D, vol. 74, p. 072003, Oct 2006.

[86] D. Michael et al., "Observation of muon neutrino disappearance with the minos detectors and the numi neutrino beam," Phys.Rev.Lett., vol. 97, p. 191801, 2006. ja href=http://www.fnal.gov/pub/today/MINOS.htmli Press Releasei/ą.

[87] M. Strait, A Measurement of Neutrino Oscillation Parameters Using Anti-fiducial Charged Current Events in MINOS. PhD thesis, University of Minnesota, 2010.

[88] A. Himmel, "Cc handscan results." MINOS-DOC 5613.

[89] J. DeJong, "Position paper on calibration of runs i-ii-iii." MINOS-DOC $6717,2010$.

[90] J. Evans, "Wrong-sign background systematic for the cc analysis." MINOSDOC 6870, 2010. 
[91] S. Coleman, "Systeamtics and alternative models." MINOS-DOC 7444$\mathrm{v} 4$.

[92] J. Mitchell, Measuring $\nu_{\mu}$ disappearance with the MINOS Experiment. PhD thesis, University of Cambridge, 2011.

[93] J. Mitchell, "Position paper on oscillation fitting for the 2010 cc analysis, version 10." MINOS-DOC 6862, version 10, 2010.

[94] J. Evans, "The 2010 charged current analysis." MINOS-DOC 7292.

[95] S. V. Cao, "Antineutrino combination in rhc and fhc modes." MINOSDOC 8598.

[96] MINOS+ collaboration, "Minos+. case for continued minos running in the numi-nova beam." MINOS-DOC 7923-v25.

[97] T. Walton, "Update on particle score." MINER $\nu$ A-DOC 6520.

[98] J. Ratchford, "Muon studies and updates." MINER $\nu$ A-DOC 6106-v7 and MINER $\nu$ A-DOC 6635-v1.

[99] T. Walton, "Muon energy reconstruction." MINER $\nu$ A-DOC 6482-v1.

[100] P. Vahle, "The nova experiment." Fermilab User's Meeting Presentation, June 2011.

[101] J. Paley et al., "Scinova: A measurement of neutrino-nucleus scattering in a narrow-band beam." Fermilab Directorate Program Planning, November 2010 PAC, October 2010.

[102] T. Adam et al., "Measurement of the neutrino velocity with the OPERA detector in the CNGS beam," 2011. 
[103] T. Hastie, The elements of statistical learning: data mining, inference, and prediction. Springer, 2009.

[104] R. Duda, P. Hart, and D. Stork, Pattern Classification. Wiley, 2001.

[105] W. Press, B. Flannery, S. Teukolsky, and W. Vettterling, Numerical Recipies : The Art of Scientific Computing, Third Edition. Cambridge University Press, 2007.

[106] S. V. Cao, "Conversations with son v. cao and various minos documents." MINOS-DOC 7575. 